\title{
Understanding the molecular mechanisms of aggression in BALB/C and TPH2-deficient mice
}

Citation for published version (APA):

Gorlova, A. (2020). Understanding the molecular mechanisms of aggression in BALB/C and TPH2deficient mice. [Doctoral Thesis, Maastricht University]. OneBook.ru. https://doi.org/10.26481/dis.20200305ag

Document status and date:

Published: 01/01/2020

DOI:

10.26481/dis.20200305ag

Document Version:

Publisher's PDF, also known as Version of record

\section{Please check the document version of this publication:}

- A submitted manuscript is the version of the article upon submission and before peer-review. There can be important differences between the submitted version and the official published version of record.

People interested in the research are advised to contact the author for the final version of the publication, or visit the DOI to the publisher's website.

- The final author version and the galley proof are versions of the publication after peer review.

- The final published version features the final layout of the paper including the volume, issue and page numbers.

Link to publication

\footnotetext{
General rights rights.

- You may freely distribute the URL identifying the publication in the public portal. please follow below link for the End User Agreement:

www.umlib.nl/taverne-license

Take down policy

If you believe that this document breaches copyright please contact us at:

repository@maastrichtuniversity.nl

providing details and we will investigate your claim.
}

Copyright and moral rights for the publications made accessible in the public portal are retained by the authors and/or other copyright owners and it is a condition of accessing publications that users recognise and abide by the legal requirements associated with these

- Users may download and print one copy of any publication from the public portal for the purpose of private study or research.

- You may not further distribute the material or use it for any profit-making activity or commercial gain

If the publication is distributed under the terms of Article $25 \mathrm{fa}$ of the Dutch Copyright Act, indicated by the "Taverne" license above, 
MAASTRICHT UNIVERSITY

\section{Understanding the molecular}

\section{mechanisms of aggression in BALB/c and TPH2-deficient mice}

\section{Anna Gorlova}


(C) A. Gorlova, Maastricht 2020

M.: OOO “Sam Poligraphist", 2019. - 326 p., ILL

Understanding the molecular mechanisms of aggression in $\mathrm{BALB} / \mathrm{c}$ and TPH2-deficient mice

ISBN 978-5-00166-023-1

Cover: Scene from Homer's The Iliad on wine-drinking bowl, c. 530 bc, from Pharsalos, Greece. National Archaeological Museum, Athens

Number of copies: 30

Order \# 89610.

Printed by «OneBook.ru» OOO "Sam Poligraphist"

129090 Moscow, Protopovsky lane 6

www.onebook.ru

All rights reserved. No parts of this book may be reproduced or transmitted in any form or by any means, without prior permission in writing by the author, or when appropriate, by the publishers of the publication. 
Understanding the molecular mechanisms of aggression in $\mathrm{BALB} / \mathrm{c}$ and TPH2-deficient mice

\section{DISSERTATION}

to obtain the degree of Doctor at Maastricht University, on the authority of the Rector Magnificus,

Prof.dr. Rianne M. Letschert

in accordance with the decision of the Board of Deans, to be defended in public

on Thursday, March $5^{\text {th }}$ 2020, at 10:00 hours

by

Anna Gorlova 


\section{Promotor:}

Prof. dr. K.P. Lesch

\section{Co-promotors:}

Dr. T. Strekalova

Prof. dr. L. Bettendorff, University of Liege, Belgium

\section{Assessment Committee:}

Prof. dr. J. Prickaerts (Chairman)

Prof. dr. Th. van Amelsvoort

Dr. A. Jahanshahi

Prof. dr. E. Budygin, Wake Forest School of Medicine, WinstonSalem, NC, USA

Dr. J. Glennon, Radboud University Nijmegen Medical Centre, Nijmegen 


\section{Table of Contents}

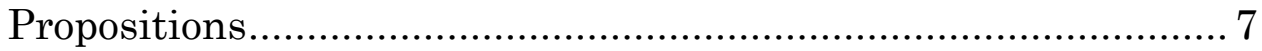

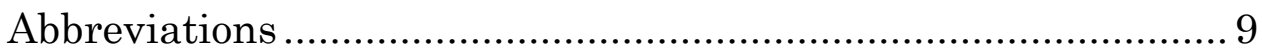

Chapter 1. General introduction ................................................ 11

1.1. Aggression and its neuroanatomical basis in human and

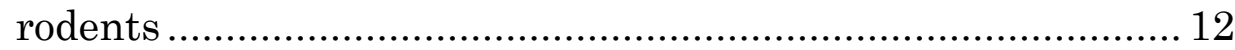

1.2. Excessive aggression as a symptom of psychiatric conditions, including depression and anxiety disorders.......... 16

1.3. Neuronal serotonin in the regulation of aggression .......... 18

1.4. Role of the glutamatergic system in the regulation of

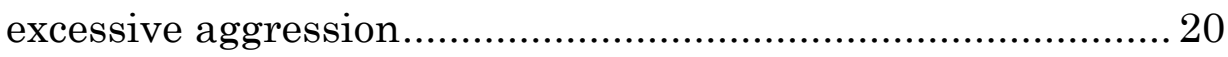

1.5. Changes in neuronal plasticity, oxidative stress and neuroinflammation as mechanisms of excessive aggression... 22

1.6. Animal models of aggression

1.7. Challenges in management of excessive aggression in the clinical setting and therapeutic potential of psychoactive antioxidant and anti-inflammatory compounds ...................... 28

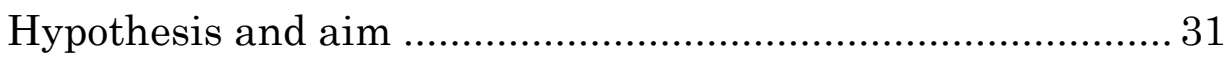

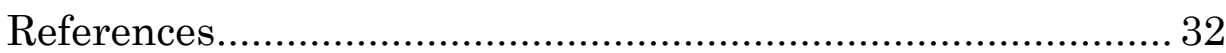

Chapter 2. Thiamine and benfotiamine counteract ultrasoundinduced aggression, normalize AMPA receptor expression and plasticity markers, and reduce oxidative stress in mice ............ 45

Chapter 3. Ultrasound stress compromises the correlates of emotional-like states and brain AMPAR expression in mice: effects of antioxidant and anti-inflammatory herbal treatment

Chapter 4. Stress-induced aggression in heterozygous TPH2 mutant mice is associated with alterations in serotonin metabolism and expression of 5-HT6 and AMPA subunit 2A receptors .......................................................................... 231

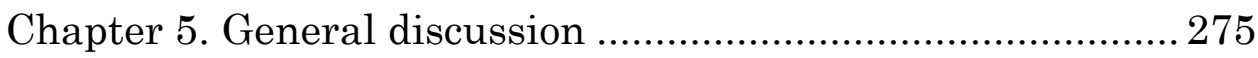


5.1. Environmental factors leading to excessive aggression: impact of emotional stress....................................................276

5.2. The role of serotonin, its receptors and Tryptophan hydroxylase-2 in pathological aggression ...............................279

5.3. Altered internalization and expression of AMPA receptor subunits in the mechanisms of neuropsychiatric disorders...284 5.4. Interplay between oxidative stress, neuroinflammation and altered neuroplasticity in excessive aggression, depressive-like and anxiety-like behavior: evidence from clinical data..........288 5.5. Antioxidant compounds as potential treatment of excessive aggression and associated conditions: perspectives of clinical use of thiamine compounds and herbal treatments...............295

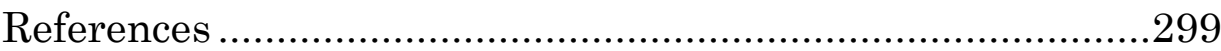

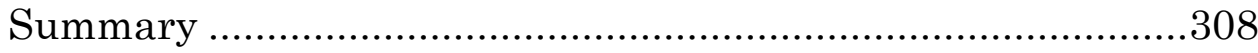

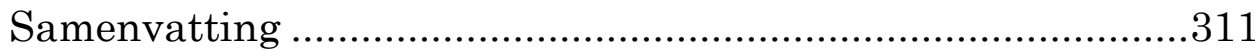

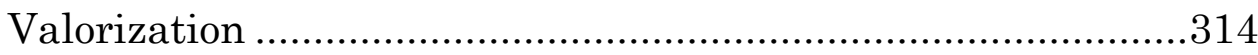

Acknowledgements............................................................... 317

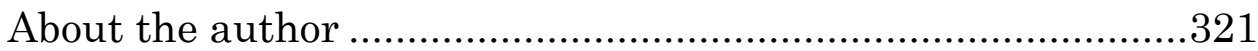




\section{Propositions}

1. Alterations in the expression of 5-HT6-R and AMPA-R subunits GluA1-3 and internalization of the latter are implicated in excessive aggression in the ultrasound mouse model of 'emotional stress' (this thesis);

2. In mice with partial genetic deficit of tryptophan hydroxylase-2 (Tph2-het), predation stress evokes aggression and changes in the expression of 5-HT6-R, GluA2 and serotonin metabolism (this thesis);

3. Ultrasound-induced aggression in $B A L B / c$ mice is associated with neuroinflammation, decreased hippocampal plasticity and oxidative stress (this thesis);

4. Compounds with antioxidant properties, including vitamin B1 (thiamine), benfotiamine and herbs, normalize ultrasound-induced aggression and accompanying molecular changes (this thesis);

5. New paradigms of aggressive behaviour, evoked by 'emotional stress' in BALB/c mice and by predation in Tph2-het mice are useful for fundamental and pre-clinical studies (valorization); 
6. Gene-environment interactions underpin manifestations of many psychopathologies;

7. The use of multiple tests and a variety of experimental conditions is the must with the validation of animal models of psychiatric disorders;

8. A balance between oxidative stress and inflammation on one hand and counteracting detoxifying and antiinflammatory factors on another hand determines healthy state;

9. Aggression unopposed becomes a contagious disease (Jimmy Carter);

10.War is sweet to those who have not experienced it (Desiderius Erasmus). 


\section{Abbreviations}

5-HIAA

5-HT

5-HTP

Akt

AMPA

BBB

BDNF

CNS

COX-2

CRP

CSF

DLPFC

DNA

ELS

GSK

$\mathrm{HAB}$

HPA

IFN

IL

iNOS

LAB

L-DOPA

MAOA

mPFC

NAD 5-hydroxyindoleacetic acid

5-hydroxytryptamine

5-hydroxytryptophan

Protein kinase B

a-amino-3-hydroxy-5-methyl-

isoxazolepropionic acid

Blood-brain barrier

Brain-derived neurotrophic factor

Central nervous system

Cyclooxigenase-2

C-reactive protein

Cerebrospinal fluid

Dorsolateral prefrontal cortex

Deoxyribonucleic acid

Early-life stress

Glycogen synthase kinase

High anxiety-like behavior

Hypothalamic-pituitary-adrenal

Interferon

Interleukin

Inducible nitric oxide synthase

Low anxiety-like behavior

L-3,4-dihydroxyphenylalanine

Monoamine oxidase A

Medial prefrontal cortex

Nicotinamide adenine dinucleotide 
NMDA

NOX

OFC

PFC

PSA-NCAM

PSD95

PTSD

RNS

ROS

SAL

SNP

SOD

SSRI

TD

TGF

$\mathrm{Th}$

Tn

TNF

TPH

Treg

$\mathrm{VMH}$

WHO
$\mathrm{N}$-methyl-D-aspartate receptor

NADPH oxidase

Orbitofrontal cortex

Prefrontal cortex

Polysialylated form of neural cell adhesion molecule

Postsynaptic density protein 95

Posttraumatic stress disorder

Reactive nitrogen species

Reactive oxygen species

Short attack latency

Single nucleotide polymorphism

Superoxide dismutase

Selective serotonin reuptake inhibitor

Thiamine deficiency

Transforming growth factor

$\mathrm{T}$ helper cell

T naïve cell

Tumor necrosis factor

Tryptophan hydroxylase

$\mathrm{T}$ regulatory cell

Ventromedial nucleus of the hypothalamus

World Health Organization 


\title{
Chapter 1
}

\author{
General introduction
}




\section{Chapter 1. General introduction}

\subsection{Aggression and its neuroanatomical basis in}

\section{human and rodents}

Aggression comprises a suite of agonistic behavioral interactions and is prevalent in most species. Aggressive behavior is considered a social behavior that may have an adaptive function, conferring biological advantages toward survival and reproduction (Lane et al., 2011). For example, it serves to protect the individual and to maintain dominance in social environments, influencing the hierarchical structure in rodents and primates (de Almeida et al., 2015). However, extreme and persistent forms of aggression are regarded as nonadaptive and pathological. Excessive aggression can result in ignoring submissiveness of an opponent, or an excessive response to provocation (Haller \& Kruk, 2006). In human aggression, inflicted harm can include physical injury, psychological and emotional trauma, and financial loss (Reiss \& Ross, 1993). The World Health Organization categorizes violent behavior among the top 20 causes of disability worldwide and this figure is likely to increase by 2030 (Vakili et al., 2015).

Data from animal and human studies have indicated several key brain regions, primarily in the limbic system, associated with aggression (Figure 1). Cortical lesions or injuries often result in disinhibited aggressive behavior (Grafman et al., 1996), and individuals with antisocial personality disorder display reductions in prefrontal gray matter (Raine et al., 2000). Subjects with impulsive aggression were shown to have lower prefrontal 
cortex (PFC) metabolic activity (Yang et al., 2008). In rodents, the PFC also has important regulatory roles in aggressive behavior (Wall et al., 2012). Activation of the medial PFC (mPFC) with optogenetic techniques was shown to inhibit inter-male aggressive behavior in mice, without affecting other behaviors. This demonstrates an important inhibitory influence of the PFC on aggression.

The amygdala mediates fear and defensive responses (Cardinal et al., 2002), and is important in the processing of emotionally adverse events. It is active in a number of social conditioning and fear paradigms (Davis \& Whalen, 2001). Patients with damage to the amygdala demonstrate impairment in the recognition of fearful facial expressions (Adolphs et al., 1994). Children with conduct disorders and prominent aggressive behavior generally have smaller PFC, amygdala and hippocampus volume (Fahim et al., 2011). In mice, inter-male aggression-related behaviors were inhibited following medial amygdala lesioning (Wang et al., 2013). Neurons of medial amygdala are active during social behaviors such as fighting and mating (Hong et al., 2014).

The dorsolateral prefrontal cortex (DLPFC) and orbitofrontal cortex (OFC) receive inputs from the amygdala and other medial temporal areas that may integrate sensory information with affective signals (Schoenbaum et al., 2003). Current theory proposes that the network between these structures and the amygdala underlie the processing of emotional 
and goal directed behavior, and that the dysfunction of any of these structures results in problems with the regulation of emotion. This can manifest as difficulties with the inhibition of aggressive behavior (Damasio et al., 1996), and violent patients often have reduced prefrontal-amygdala and prefrontal-striatal connectivity (Cunha-Bang et al., 2017). Thus, an imbalance between the regulatory influence of the $\mathrm{PFC}$ and the responsivity of the amygdala are chiefly implicated in excessive aggression (Siever, 2008).

Neural circuits that modulate aggressive behavior also include the hippocampus and hypothalamus. Abnormalities in hippocampal function have been identified in antisocial and violent individuals. Specifically, atypical hippocampal asymmetries that disrupt prefrontal-hippocampal circuitry may result in emotion dysregulation and poor contextual fear conditioning (Raine et al., 2004), since hippocampal neurons have robust projections, that originate from hippocampal CA1 and terminate in the orbital and medial frontal cortices. In mouse studies, stress-induced attack behavior was shown to be associated with activation of ventral hippocampus neurons (Chang, Gean, 2019). Additionally, pyramidal neurons in the CA2 region of the hippocampus, which are important for social memory, promote social aggression in mice (Leroy et al., 2018). In the hypothalamus, electrical stimulation of ventromedial nucleus (VMH) and lateral hypothalamus result in increased aggressive behavior in animals (Siegel et al., 1990). The VMH 
receives inputs from the lateral hypothalamus as well as the cortical and basolateral amygdala, which modulate the expression and duration of aggressive behaviors (Falkner et al., 2008). The central, lateral, and basal nuclei of the amygdala facilitate aggressive attacks (Gregg, Siegel, 2001). Human studies also suggest that the hypothalamus is related to the control of aggressive behavior (Ramamurthi, 1998). Neuroimaging studies show that the hypothalamus is more active in individuals with aggressive features and domestic violence offenders (George et al., 2004).

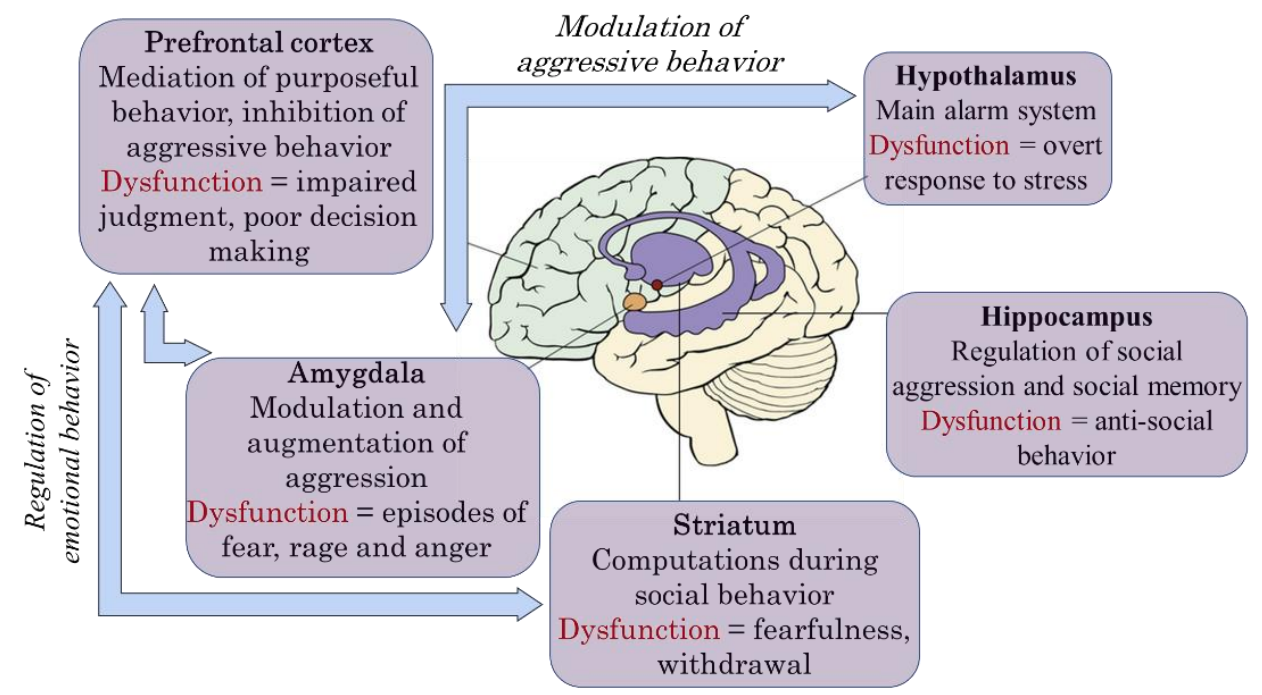

Figure 1. Brain structures involved in regulation of aggressive behavior and results of its dysfunction. The prefrontal cortex exerts an inhibitory effect on aggression; the hypothalamus, hippocampus and striatum are related to the control of aggressive behavior, while the amygdala mediates defensive reactions. Reduced prefrontal-amygdala and prefrontal-striatal connectivity results in excessive aggression; interconnection between the amygdala and hypothalamus underlie the regulation of the extent of aggressive behaviors (adapted from Stuart, 2013). 


\subsection{Excessive aggression as a symptom of psychiatric}

conditions, including depression and anxiety disorders

When aggression in adults is not a response to a clear threat, it is considered a sign of mental disorder (Shelton, 2001). In fact, there is comorbidity between aggression and mental illness, and psychiatric disorders may, although rarely, be associated with violence (Swanson et al., 1990). Paranoid schizophrenia is most frequently associated with social withdrawal, impaired social cognition and aggressive behavior (Citrome \& Volavka, 2001). Personality disorder symptoms, more specifically, anti-social personality disorder, often include aggressive urges and threats (Scott et al., 2017).

Aggression is a key risk factor associated with substance use. Adults with substance use disorder often have histories of aggressive behaviors (Morie et al., 2014) associated with impulsivity and attention problems (Ernst et al., 2006). According to the World Health Organization, alcohol consumption is more strongly associated with aggressive behavior than the use of any other psychotropic substance (WHO, 2017). The relationship between alcohol use and aggressive behavior is well documented in epidemiological studies (Darke et al., 2010), and wide-spread alcohol use and abuse results in considerable socioeconomic costs every year (Budygin \& Weiner, 2015). Posttraumatic stress disorder (PTSD) is closely associated with aggressive behavior (Angkaw et al., 2013). Trauma influences anger and aggression through social information processing mechanisms (Taft et al., 
2017). Approximately $70 \%$ of patients with dementia suffer from behavioral disorders that include anxiety, agitation and aggression (Herrmann et al., 1996), wherein excessive aggression usually occurs in advanced dementia (Müller-Spahn et al., 2003).

Disturbances of aggression regulation are a frequent component of mood disorders (Botsis et al, 1997), including depression and comorbid generalized anxiety. Though depression is a broad and heterogeneous diagnostic category, its clinical presentation is often accompanied with both anger and anxiety (Llorca et al., 2016). Irritability and anger associated with depression and anxiety can result in aggression, and depressed patients may exhibit aggressive behavior as part of their symptomatology. Anger attacks have been observed in $30-40 \%$ of depressed patients, both in major depression and dysthymia (Fava et al, 1993), and in numerous studies hostile behavior has been a key symptom in depressed adolescents (Benarous et al., 2014).

Panic disorder is also characterized by increased anger outbursts (Korn et al, 1997), and both preclinical and clinical findings demonstrated that anxiolytic drugs can reduce male aggressive behavior in rodents (Kudryavtseva \& Bondar, 2002) and humans (Haller et al., 2007). Anxiety/aggression-driven depression has even been proposed as a new subtype of depression, in which aggression dysregulation is not only a symptom, but a pacemaker of disorder progression (Van Praag, 
2001). Altogether, major mental illness is considered a significant risk factor for violent behavior (Swanson et al., 1990) (Figure 2).

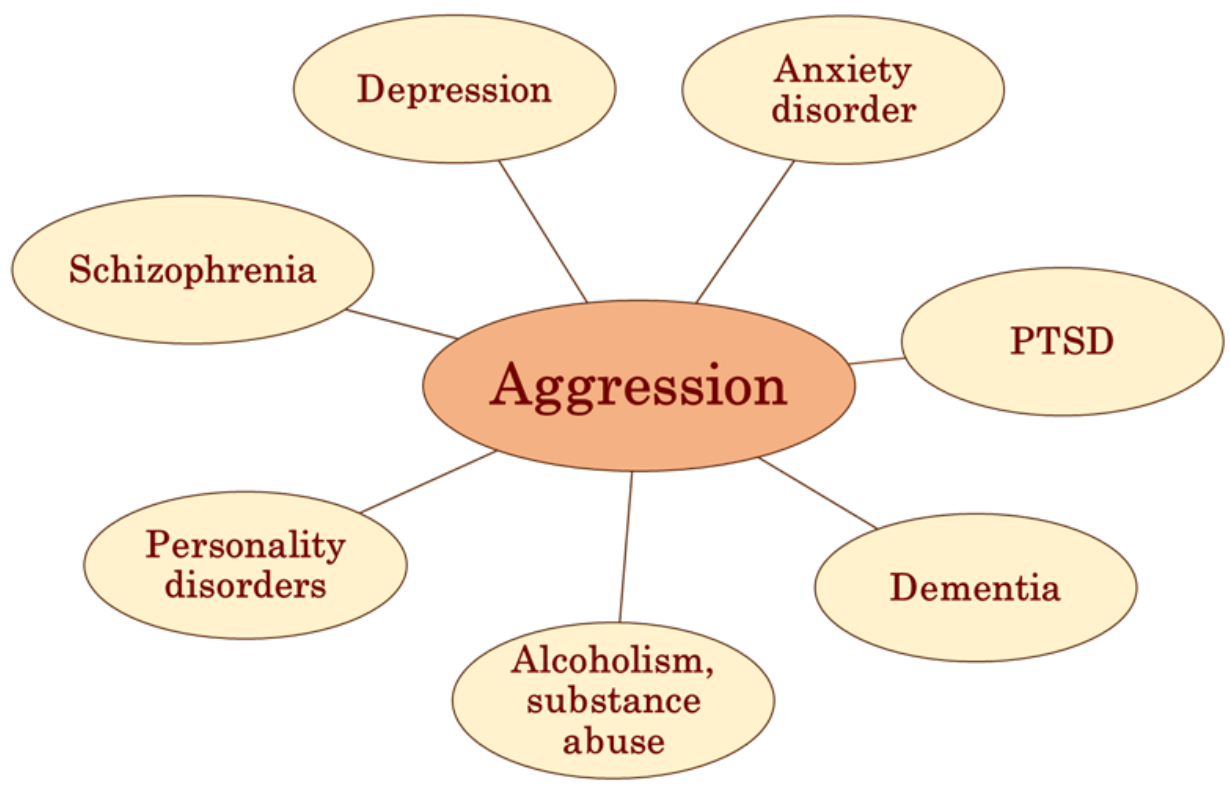

Figure 2. Excessive aggression as a syndrome of numerous psychiatric disorders. Aggressive behavior is associated with psychotic and personality disorders, schizophrenia, depression, anxiety disorder, PTSD, dementia and alcoholism.

1.3. Neuronal serotonin in the regulation of aggression

Serotonin (5-hydroxytryptamine, 5-HT), a monoamine neurotransmitter derived from L-tryptophan, is considered an important regulator of mood (Kumar et al., 2010). It has been suggested as the primary determinant of inter-male aggression (Nelson \& Chiavegatto, 2001), and aberrant synthesis, or 
response to 5-HT within the central nervous system (CNS) can result in inappropriate dampening of reactive distress, self-injury or interpersonal violence (Glick, 2015). Findings from both preclinical and clinical studies have associated dysregulation of 5-HT release, signaling and metabolism with acts of violence or suicide (Mann et al., 2001).

It is known that 5-HT facilitates prefrontal cortical regions, involved in modulating and suppressing the emergence of aggressive behaviors (Figure 3). Seven subtypes of 5-HT receptors have been identified that mediate both excitatory and inhibitory inputs in a number of brain regions associated with aggression, emotionality and cognition (Siever, 2008). Serotonergic projections from the raphe nuclei of the brainstem to the PFC, OFC, amygdala, and nucleus accumbens modulate the individual's reactivity to insult or threat (Bortolato et al., 2013). Destruction of the dorsal raphe by fenclonine, an irreversible inhibitor of tryptophan hydroxylase (TPH), increase aggression in mice (Kostowski et al., 1975). Thus, deficiencies in serotonergic innervation of these regions can result in disinhibited aggression. Both animal and human research have shown that aggressive individuals have lower levels of the neurotransmitter 5-HT (Virkkunen et al., 1995). Numerous studies have shown that selective 5-HT reuptake inhibitors (SSRIs) reduce impulsive aggression in patients with personality disorder (Coccaro \& Kavoussi, 1997) and other aggression-related psychopathologies, including depression (Coccaro et al., 2015). 
Enzymes associated with central 5-HT metabolism may also be implicated in pathological aggression, in particular monoamine oxidase A (MAOA) and TPH2 (Popova, 2008). Deficiency of MAOA, an enzyme which degrades amine neurotransmitters, including serotonin, was shown to lead to impulsive aggression in animals and humans. A nonsense mutation of the MAOA gene results in Brunner syndrome, which is characterized by violent behaviors in response to relatively minor stressors (Brunner et al., 1993; Bortolato et al., 2013). The synthesis of 5-HT depends on TPH with the TPH2 isoform being predominant in the brain (Carkaci-Salli et al., 2011; Gutknecht et al., 2009). Tph2-deficient mice displayed altered sociability and untypically high levels of aggressive behavior in different studies (Kästner et al., 2019; Lesch et al., 2012; Gutknecht et al., 2015; Weidner et al., 2019). Collectively, it is clear that alterations in central serotonergic homeostasis may result in excessive levels of aggression.

\subsection{Role of the glutamatergic system in the regulation of excessive aggression}

Altered levels of glutamate are also associated with increased aggressive behavior, particularly impulsive aggression (Brodie et al., 2016) (Figure 3). Glutamatergic antagonism leads to behavioral disinhibition, and NMDA and AMPA receptors antagonists have been suggested to have pro-aggressive effects (Newman et al., 2012). Social dominance is controlled by synaptic 
efficacy in the medial prefrontal cortex, in which AMPA receptors play a key role. A strong correlation between social dominance and AMPA receptor phosphorylation was found, that suggests that AMPA receptor phosphorylation in the PFC may serve as a biomarker of social dominance related to stress (Park et al., 2008). Several studies indicated a special role of GluA receptor subunits in the regulation of aggressive behavior in rodents. Inhibition of GluA1 and GluA5 activity by the specific antagonists reduced isolation-induced aggression in mice (Navarro et al., 2008). Naïve male mice deficient for GluA subunits resulted in a 30\% reduction in hippocampal AMPA binding. These mice were less aggressive than wild-type littermates in several paradigms of agonistic behavior, while exhibiting similar basic behavior and reflexes (Vekovischeva et al., 2004). In Turku Aggressive mice, selected for high scores of aggression, AMPA antagonists suppressed aggressive manifestation, while in Turku Non-Aggressive mice these antagonists, in contrast, activated aggression, that supports the hypothesis that AMPA receptors are involved in the modulation of agonistic impulsive behavioral patterns (Vekovischeva et al., 2007).

In human studies, glutamate concentration in the cerebrospinal fluid (CSF) correlated significantly with measures of aggression and impulsivity, suggesting a positive relationship between glutamate levels and aggression in humans (Coccaro et al., 2013). Increased glutamate metabolism was found in the anterior cingulate cortex of patients with borderline personality 
disorder, which is often accompanied with aggression. This also lends support to the hypothesis that increased glutamate levels may act as a marker of impulsivity (Hoerst et al., 2010).

\subsection{Changes in neuronal plasticity, oxidative stress} and neuroinflammation as mechanisms of excessive aggression

Both an aggressive experience and the expectation of an aggressive event are associated with increases in inflammatory cytokines, which can be the result of sympathetic activation (Bierhaus et al., 2003) (Figure 3). Mice bred for high aggression had increased cytokine levels, and knockout of both tumor necrosis factor (TNF) receptors 1 and 2 resulted in the absence of aggressive behavior (Patel et al., 2010). Deletion of TNF receptors, TNF-R1 and TNF-R2, reduced the duration of aggressive behaviors in the resident-intruder test in male mice, which is in line with findings from human studies in which serum TNF is increased in highly aggressive individuals (Patel et al., 2010). Glycogen synthase kinase-3 (GSK-3) has been demonstrated to promote inflammation, as well as aggressive and depression-like behaviors in rodents, and reduced expression of either GSK-3 isoform results in decrease aggressive behaviors in mice (Beaulieu et al., 2008).

Human studies show that individuals with excessive aggression display heightened inflammatory cytokine levels and dysregulated immune responses, and comorbidity of depression 
and aggression correlated with stronger immune dysregulation (Takahashi et al., 2018). Elevated aggressive traits were associated with increased serum TNF (Suarez et al., 2002) and the inflammatory marker C-reactive protein (CRP) (Coccaro et al., 2006). CRP has been suggested as a predictor of the risk of aggressive behavior among psychiatric inpatients (Martone, 2019). It has been reported that immunotherapy to treat patients with hepatitis $\mathrm{C}$ by chronic administration of interferon alpha (IFN-a) increases irritability and anger/hostility in some patients (Lotrich et al., 2013). Furthermore, pro-inflammatory cytokines IL-2, TNF and anti-inflammatory factor IL-4, IL-5 and IL-10 were significantly elevated in patients with excessive aggression and PTSD that underwent early life trauma (Mommersteeg et al., 2008). Marital couples show increases in plasma IL-6 and TNF after conflict interactions, and these increases in cytokines were larger in couples who showed higher hostile behaviors during their conflict interactions (Kiecolt-Glaser et al., 2005). IL-6 levels were also significantly higher in participants with intermittent explosive disorder (Coccaro et al., 2014). These studies cumulatively suggest a role of inflammation and immune dysregulation in the pathophysiology of aggression.

Oxidative stress is another pathophysiological mechanism underlying excessive aggression (Figure 3). Measures of oxygen radicals in Long-Evans rats were shown to correlate with their aggression scores (Patki et al., 2015), and intracellular redox status of peripheral blood granulocytes correlated significantly 
with the aggressive behavior levels of adult male mice (Rammal et al., 2010). Impaired antioxidant defense also has a direct effect on aggressive behavior. Mice deficient in copper-zinc superoxide dismutase (SOD1) that express 50\% of this antioxidant enzyme, are more aggressive than wild-type males (Garratt \& Brooks, 2015). Human studies suggest a positive relationship between plasma markers of oxidative stress 8-hydroxy-2'-deoxyguanosine and 8-isoprostane and aggression in human subjects (Coccaro et al., 2016). These oxidative stress markers were also elevated in adult subjects with personality disorder that displayed aggressive traits (Fanning et al., 2015).

Neural plasticity is the change in structural and functional connections within the brain in response to environmental circumstances and experiences and allows individual adjustment to extraneous input (Cicchetti \& Curtis, 2006) (Figure 3). Specifically, neural plasticity in the hippocampus and amygdala is crucial to adaptive social behavior, including aggression (Arendt et al., 2012). Impaired neurogenesis and atrophy of dendrites in the hippocampus after chronic stress correlate with increased anxiety and aggression in rodents, while the same stressor causes dendritic growth in the amygdala (McEwen, 2007). Expression of the brain-derived neurotrophic factor (BDNF) gene is required for motivational aspects of social interaction (Berton et al., 2006). In human, mutation of the human BDNF gene (Val66Met; G196A) is correlated with a 
higher susceptibility to stress-induced affective disorders and heightened tress responses (Colzato et al., 2011).

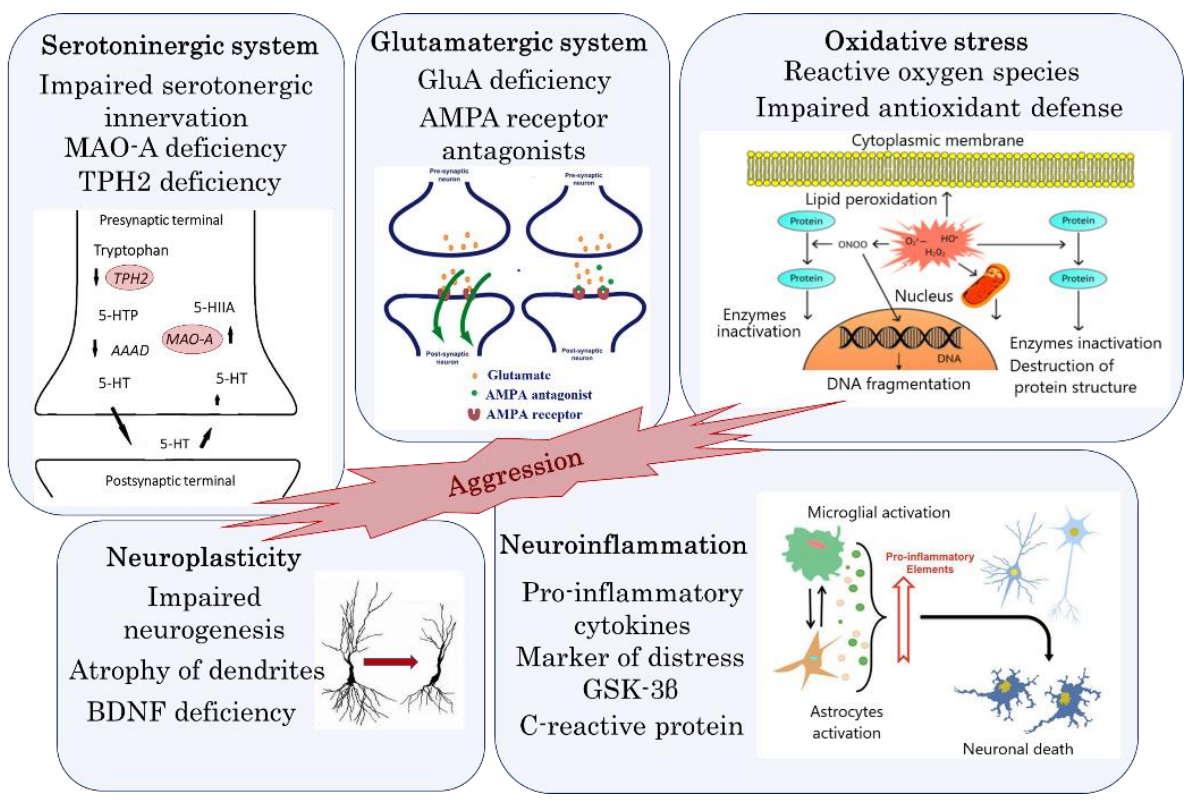

Figure 3. Mechanisms of excessive aggression. Excessive aggression is associated with 5-HT deficiency, decreased functioning of glutamatergic system, oxidative stress, neuroinflammation and impaired neuroplasticity (adapted from Kulikov et al., 2018; Egervani, 2016; Morales et al., 2014; Jia et al., 2010).

\subsection{Animal models of aggression}

The development of animal models can be a powerful tool to investigate the pathophysiology of excessive aggression and find new effective therapies (Bortolato et al., 2013). Maladaptive aggressive behavior in rodents is defined by a short latency to initiate the attack, high duration and high intensity of attacks, which lead to significant injury, lack of species-normative 
behavioral structure and insensitivity to signals of submission (Haller \& Kruk, 2006).

Role of genetic factors in excessive aggression can be investigated with the use of transgenic animal models. Reduced scores of aggression have been found in male knockout mice lacking the long form of the dopamine D2 receptor (Vukhac et al., 2001), the a-isoform of the oestrogen receptor (Scordalakes \& Rissman, 2003), the arginine vasopressin V1b receptor (Wersinger et al., 2002) or dopamine B-hydroxylase (Marino et al., 2005). In contrast, elevated aggression was shown in mice lacking 5-HT1B receptor (Saudou et al., 1994), dopamine transporter (Rodriguiz et al., 2004), nitric oxide synthase (Nelson et al., 1995), or MAOA (Cases et al., 1995).

Other common models of escalated aggression are mouse and rat strains selectively bred for high aggression. One such model are mice bred for short attack latency (SAL), which display elevated aggression in conjunction with low brain 5-HT levels and reduced reuptake transporter activity (van Oortmerssen \& Bakker, 1981). Excessive and abnormal forms of aggression are also demonstrated in Turku Aggressive mice (Sluyter et al., 2003) and the North Carolina 900 and 100 mice (Natarajan et al., 2009). Selectively breed Wistar rats with low anxiety-like behavior (LAB), initially used as controls to rats with high anxiety-like behavior (HAB) also display high and abnormal forms of aggression (Landgraf et al., 2007). The prairie vole (Microtus ochrogaster) has recently emerged as a new animal model for 
investigating the neurobiology of escalated aggression and violence, since ethologically their mating is accompanied by aggressive behavior directed toward both male and female conspecifics (Gobrogge et al., 2014).

Mice exposed to chronic mild stress paradigm showed increased offensive and aggressive behaviors in the residentintruder test and social dominance tube test (Yang et al., 2015). The social defeat paradigm has also been shown to induce excessive aggression in dominant males (Golden et al., 2011). Early life stress is another major risk factor for excessive aggression, as it results in maldevelopment of the hypothalamicpituitary-adrenal (HPA) axis, one of the major systems of regulating physiological responses to stress (Toth et al., 2008; Veenema et al., 2006). Excessive aggression can be modeled in adrenalectomized rats maintained by low-level glucocorticoid replacement therapy (Haller et al., 2001). Moreover, aggression is escalated by acute alcohol doses or during withdrawal from prolonged exposure to repeated high alcohol doses, which is modelled in mice, rats and monkeys (Faccidomo et al., 2008). Deprivation of an expected reward can also induce a state of hyper-aggression (Neumann et al., 2010). Thus, a large variety of animal models of aggression exist (Figure 4). However, existing models fail to utilize emotional stress as a trigger for aggressive behavior, which is a frequent cause of excessive aggression in humans. 

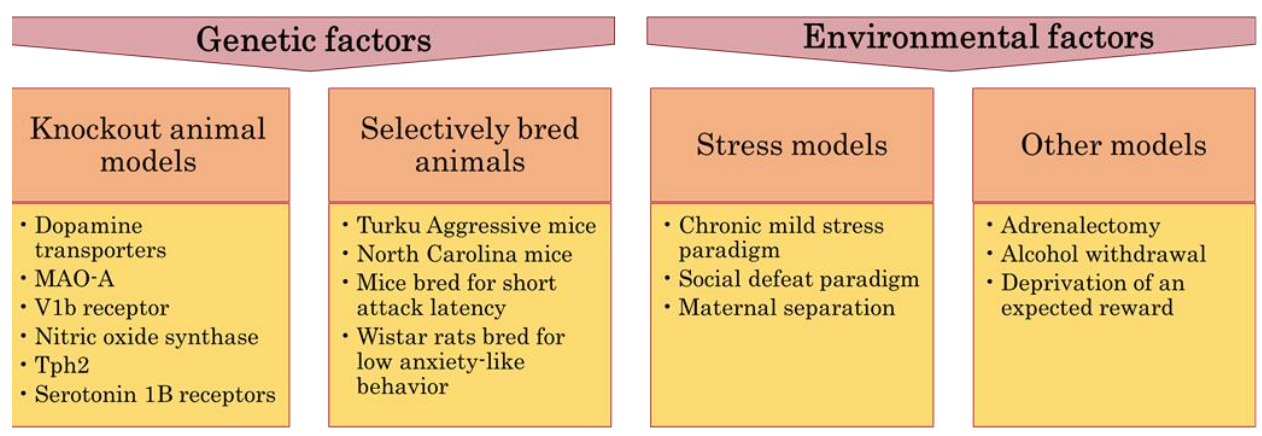

Figure 4. Animal models of aggression. Classification of excessive aggression models in rodents includes transgenic animals, selectively bred animals, stress models and other models, such as adrenalectomy and alcohol withdrawal.

\subsection{Challenges in management of excessive} aggression in the clinical setting and therapeutic potential of psychoactive antioxidant and antiinflammatory compounds

Although pharmacotherapy is often used in the treatment for excessive aggression, it usually is not treated specifically, but in conjunction with other psychiatric disorders (Stanford et al., 2009). As a consequence, medications used in the treatment of acute aggression often do to not have specific effects on aggression, but have general sedative effects (Lane et al., 2011). Most commonly benzodiazepines, e.g., lorazepam, and antipsychotic medication are used, alone or in combination (Gillies et al., 2005).

Although treatment of acute aggression can be relatively effective, there are few controlled trials of medication for chronic 
aggressive behavior, where sedation is not an acceptable side effect. There is some data to support a specific anti-aggressive effect of low doses of second generation antipsychotic medications (Ballard et al., 2009). For example, risperidone was shown to have a significant improvement in aggression in patients with dementia or schizophrenia (Aleman et al., 2001).

Anticonvulsant compounds are another widely studied class of drugs for the treatment of chronic aggression (Lane et al., 2011). Phenytoin (Donovan et al., 2000) and valproate (Stanford et al., 2005) were studied in placebo trials that suggested their effectiveness in treatment of pathological aggression. Newer anticonvulsants Gabapentin (Cherek et al., 2004), Tiagabine (Lieving et al., 2008) and Topirimate (Lane et al., 2009) have been reported to reduce aggression in both animal and human studies.

SSRIs are widely used clinically for the treatment of aggression, and fluoxetine is the most studied of them (Salzman et al., 1995). Numerous studies reported decreased anger and verbal aggression in fluoxetine-treated patients with personality disorders (Rinne et al., 2002) and even in patients with high hostility scores but who were otherwise healthy (Kamarck et al., 2009). Randomized clinical trials suggested the effectiveness of non-selective beta-adrenoceptor and partial 5-HT1A receptor antagonists propranolol and pindolol in the management of aggression and agitation in patients with traumatic brain injury (Fleminger et al., 2006). However, only large doses of compounds 
were effective in these trials, and major side effects, such as bradycardia, were observed.

The usefulness of antihypertensive drugs and psychostimulants was demonstrated in some cases of excessive aggression, but only marginal benefits were observed (Fava, 1997). Risperidone, an atypical antipsychotic drug that blocks dopamine and 5-HT receptor systems, was shown to be effective for severe aggression in adolescents with disruptive behavior disorders (Buitelaar et al., 2001).

Since increased aggression is associated with oxidative stress and neuroinflammation, some recent studies have suggested the therapeutic potential of psychoactive antioxidant and anti-inflammatory compounds. In mice, treatment with lithium inhibits GSK-3 and therefore has anti-inflammatory effects. This is associated with significantly reduced aggression, impulsivity and depression traits (Beurel \& Jope, 2014). In human studies, there is some evidence that lithium is effective in patients with affective or impulsive aggression (Malone et al., 1998). However, lithium has side effects that include tremor, sedation, nausea, polyuria and risk of neurotoxicity (Glenn et al., 1989).

Decreased levels of endogenous antioxidants such as glutathione and superoxide dismutase lead to an increase in oxidative stress, which in turn produces anxiogenic behavior and aggression in mice (Masood et al., 2008). The potential of antioxidant treatment in the management of aggression has been 
hypothesized in few studies. Ascorbic acid, beta carotene and vitamin $\mathrm{E}$ showed dose dependent effects and significantly reduced the tail rattling, attacking and biting response in the model of L-DOPA-induced aggression (Hira et al., 2017).

\section{Hypothesis and aim}

In the current study, we used two models of aggression, the ultrasound model of "emotional stress" and mice with a heterozygous inactivation of TPH2 (Tph2+/- or Tph2-het). Since stress can exacerbate brain 5-HT deficiency and cause changes in aggressive behavior, we hypothesize that Tph2-het mice will display increased aggressive behavior, showing a similar behavioral profile to that of Tph2-null mutants. Given recently demonstrated anti-aggressive and anti-stress effects of compounds that target AMPA and 5-HT6 receptors, we investigated whether or not the expression of these molecules' changes in two mouse paradigms of stress-induced aggression. Using chronic ultrasound exposure, a model of emotional stress, we also aimed to study a relationship between measures of aggressive behavior and the expression of markers of brain oxidative stress, plasticity and neuroinflammation. In addition, we studied, whether antioxidant thiamine compounds and herbs with antioxidant activity can counteract behavioral and molecular changes. Furthermore, we investigated whether changes in gene expression in stressed Tph2-het mice are related to excessive aggression and 5-HT metabolism in brain. 


\section{References}

Adolphs R., Tranel D., Damasio H., Damasio A. Impaired recognition of emotion in facial expressions following bilateral damage to the human amygdala. Nature. 1994, 327: 669-672.

Aleman A., Kahn R.S. Effects of the atypical antipsychotic risperidone on hostility and aggression in schizophrenia: a meta-analysis of controlled trials. Eur Neuropsychopharmacol. 2001, 11: 289293.

Angkaw A.C., Ross B.S., Pittman J.O., Kelada A.M., Valencerina M.A., Baker D.G. Post-traumatic stress disorder, depression, and aggression in OEF/OIF veterans. Mil Med. 2013, 178: 1044-1050.

Arendt D.H., Smith J.P., Bastida C.C., Prasad M.S., Oliver K.D., Eyster K.M., Summers T.R., Delville Y., Summersa C.H. Contrasting hippocampal and amygdalar expression of genes related to neural plasticity during escape from social aggression Physiol Behav. 2012, 107(5): 670-679.

Ballard C.G., Gauthier S., Cummings J.L, Brodaty H., Grossberg G.T., Robert P., Lyketsos C.G. Management of agitation and aggression associated with Alzheimer disease. Nat Rev Neurol. 2009, 5: 245-255.

Beaulieu J.M., Zhang X., Rodriguiz R.M., Sotnikova T.D., Cools M.J., Wetsel W.C., Gainetdinov R.R., Caron M.G. Role of GSK3 beta in behavioral abnormalities induced by serotonin deficiency. Proc Natl Acad Sci USA. 2008, 105 :1333-1338.

Benarous X., Guedj M.J., Braitman A., Gallois E., Lana P. The link between aggressive behavior and depression in adolescence. A cross-sectional study conducted in the psychiatric emergency unit at the Sainte-Anne hospital. Encephale. 2014, 40: 439-446.

Berton O., McClung C.A., Dileone R.J., Krishnan V., Renthal W., Russo S.J., Graham D., Tsankova N.M., Bolanos C.A., Rios M., Monteggia L.M., Self D.W., Nestler E.J. Essential role of BDNF in the mesolimbic dopamine pathway in social defeat stress. Science. 2006, 311: 864-868.

Beurel E., Jope R.S. Inflammation and lithium: clues to mechanisms contributing to suicide-linked traits. Transl Psychiatry. 2014, 4: e488.

Bierhaus A., Wolf J., Andrassy M., Rohleder N., Humpert P.M., Petrov D., Ferstl R., von Eynatten M., Wendt T., Rudofsky G., Joswig M., Morcos M., Schwaninger M., McEwen B., Kirschbaum C., Nawroth P.P. A mechanism converting psychosocial stress into 
mononuclear cell activation. Proc. Natl. Acad. Sci. U.S.A. 2003, 100, 1920-1925.

Bortolato M., Pivac N., Seler D.M., Perkovic M.N., Pessia M., Giovanni $\mathrm{G}$. The role of serotonergic system at the interface of aggression and suicide. Neuroscience 2013, 236: 160-185.

Botsis A.F., Soldatos C.R., Stefanis C.N., 1997. Suicide: Biopsychosocial Approaches. Amsterdam, the Netherlands: Elsevier.

Brodie M.J., Besag F., Ettinger A.B., Mula M., Gobbi G., Comai S., Aldenkamp A.P., Steinhoff B.J. Epilepsy, antiepileptic drugs, and aggression: an evidence-based review. Pharmacological Reviews. 2016, 68: 563-602.

Brunner H.G., Nelen M., Breakefield X.O., Ropers H.H., van Oost B.A. Abnormal behavior associated with a point mutation in the structural gene for monoamine oxidase A. Science. 1993, 262: $578-580$.

Budygin E.A., Weiner J.L., 2015. Exploring the Neurochemical Basis of Alcohol Addiction-Related Behaviors: Translational Research. Transl Biomed. 6 (Suppl Spec).

Buitelaar J.K., van der Gaag R.J., Cohen-Kettenis P., Melman C.T. A randomized controlled trial of risperidone in the treatment of aggression in hospitalized adolescents with subaverage cognitive abilities. J Clin Psychiatry. 2001, 62: 239-248.

Cardinal R.N., Parkinson J.A., Hall J., Everitt B.J. Emotion and motivation: the role of the amygdala, ventral striatum, and prefrontal cortex. Neurosci Biobehav. 2002, 26: 321-352.

Carkaci-Salli N., Salli U., Kuntz-Melcavage K.L., Pennock M.M., Ozgen H., Tekin I., Freeman W.M., Vrana K.E. TPH2 in the ventral tegmental area of the male rat brain. Brain res bulletin. 2011, 84: $376-380$.

Cases O., Seif I., Grimsby J., Gaspar P., Chen K., Pournin S., Müller U., Aguet M., Babinet C., Shih J. C., De Maeyer E. (1995). Aggressive behavior and altered amounts of brain serotonin and norepinephrine in mice lacking MAOA. Science 268, 1763176610.1126/science.7792602

Cherek D.R., Tcheremissine O.V., Lane S.D., Pietras C.J. Acute effects of gabapentin on laboratory measures of aggressive and escape responses of adult parolees with and without a history of conduct disorder. Psychopharmacology (Berl). 2004, 171: 405-412.

Cicchetti D., Curtis W.J. The developing brain and neural plasticity: Implications for normality, psychopathology, and resilience. Developmental Psychopathology Developmental Neuroscience. 2nd ed. Vol. 2. New York: Wiley; 2006. 
Citrome L., Volavka J. Aggression and violence in patients with schizophrenia. In: Hwang MY, Bermanzohn PC, editors. Schizophrenia and comorbid conditions: Diagnosis and treatment clinical practice. American Psychiatric Publishing; Washington, DC: 2001. p. xvii.

Coccaro E.F. Association of C-reactive protein elevation with trait aggression and hostility in personality disordered subjects: a pilot study. J Psychiatr Res. 2006, 40 :460-465.

Coccaro E.F., Fanning J.R., Phan K.L., Lee R. Serotonin and impulsive aggression. CNS Spectr. 2015, 20: 295-302.

Coccaro E.F., Kavoussi R.J. Fluoxetine and impulsive aggressive behavior in personality-disordered subjects. Arch Gen Psychiatry. 1997, 54: 1081-1088.

Coccaro E.F., Lee R., Coussons-Read M. Elevated plasma inflammatory markers in individuals with intermittent explosive disorder and correlation with aggression in humans. JAMA Psychiatry. 2014, 71: 158-165.

Coccaro E.F., Lee R., Gozal D. Elevated Plasma Oxidative Stress Markers in Individuals With Intermittent Explosive Disorder and Correlation With Aggression in Humans. Biol Psychiatry. 2016, 79: 127-135.

Coccaro E.F., Lee R., Vezinab P. Cerebrospinal fluid glutamate concentration correlates with impulsive aggression in human subjects. J Psychiatr Res. 2013, 47: 1247-1253.

Colzato L.S., Van der Does A.J., Kouwenhoven C., Elzinga B.M., Hommel B. BDNF Val(66)Met polymorphism is associated with higher anticipatory cortisol stress response, anxiety, and alcohol consumption in healthy adults. Psychoneuroendocrinology. 2011, 36: 1562-1569.

Cunha-Bang S., Fisher P.M., Hjordt L.V., Perfalk E., Skibsted A.P., Bock C., Baandrup A.O., Deen M., Thomsen C., Sestoft D.M., Knudsen G.M. Violent offenders respond to provocations with high amygdala and striatal reactivity. Soc Cogn Affect Neurosci. 2017, 12: 802-810.

Damasio A.R. The somatic marker hypothesis and the possible functions of the prefrontal cortex. Philosophical Transactions of the Royal Society of London. Series B: Biological Sciences. 1996, 351: 1413-1420.

Darke S. The toxicology of homicide offenders and victims: A review. Drug Alcohol Rev. 2010, 29: 202-215.

Davis M., Whalen P.J. The amygdala: vigilance and emotion. Mol Psychiatry. 2001, 6: 13-34. 
de Almeida R.M., Cabral J.C., Narvaes R. Behavioural, hormonal and neurobiological mechanisms of aggressive behaviour in human and nonhuman primates. Physiol Behav. 2015, 143: 121-135.

Donovan S.J., Stewart J.W., Nunes E.V., Quitkin F.M., Parides M., Daniel W., Susser E., Klein D.F. Divalproex treatment for youth with explosive temper and mood lability: a double-blind, placebocontrolled crossover design. American Journal of Psychiatry. 2000, 157: 818-820.

Egervari G. AMPA Receptor Plasticity in the Nucleus Accumbens Mediates Withdrawal-Related Negative-Affective States. J Neurosci. 2016, 36: 10505-10507.

Ernst M., Luckenbaough D.A., Moolchan E.T., Leff M.K., Allen R., Eshel N., London E.D., Kimes A. Behavioral predictors of substance-use initiation in adolescents with and without Attention-Deficit/Hyperactivity Disorder. Pediatrics. 2006, 117: 2030-2039.

Faccidomo S., Bannai M., Miczek K.A. Escalated aggression after alcohol drinking in male mice: dorsal raphe and prefrontal cortex serotonin and 5-HT1B receptors. Neuropsychopharmacology. 2008, 33: 2888-2899.

Fahim C., He Y., Yoon U., Chen J., Evans A., Pérusse D. Neuroanatomy of childhood disruptive behavior disorders. Aggress Behav. 2011, 37: 326-337.

Falkner A.L., Lin D. Recent advances in understanding the role of the hypothalamic circuit during aggression. Front Syst Neurosci. 2014, 8: 1-14.

Fanning J.R., Lee R., Gozal D., Coussons-Read M., Coccaro E.F. Childhood trauma and parental style: Relationship with markers of inflammation, oxidative stress, and aggression in healthy and personality disordered subjects. Biol Psychol. 2015, 112: 56-65.

Fava M. Psychopharmacologic treatment of pathologic aggression. Psychiatr Clin North Am. 1997, 20: 427-451.

Fava M., Rosenbaum J.F., Pava J.A., McCarthy M.K., Steingard R.J., Bouffides E. Anger attacks in unipolar depression, Part 1: Clinical correlates and response to fluoxetine treatment. Am J Psychiatry. 1993, 150: 1158-1163.

Fleminger S., Greenwood R.J., Oliver D.L. Pharmacological management for agitation and aggression in people with acquired brain injury. Cochrane Database Syst Rev. 2006, 4: CD003299. 
Garratt M., Brooks R.C. A genetic reduction in antioxidant function causes elevated aggression in mice. J Exp Biol. 2015, 218: 223227.

George D.T., Rawlings R.R., Williams W.A., Phillips M.J., Fong G., Kerich M., Momenan R., Umhau J.C., Hommer D.A. Select group of perpetrators of domestic violence: evidence of decreased metabolism in the right hypothalamus and reduced relationships between cortical/subcortical brain structures in position emission tomography. Psychiatry Res. 2004, 130: 11-25.

Gillies D., Beck A., McCloud A., Rathbone J. Benzodiazepines alone or in combination with antipsychotic drugs for acute psychosis. Cochrane Database Syst Rev. 2005, 4: CD003079.

Glenn M.B., Wroblewski B., Parziale J., Levine L., Whyte J., Rosenthal M. Lithium carbonate for aggressive behavior or affective instability in ten brain-injured patients. Am J Phys Med Rehabil. 1989, 68: 221-226.

Glick A.R. The role of serotonin in impulsive aggression, suicide, and homicide in adolescents and adults: a literature review. Int $\mathrm{J}$ Adolesc Med Health. 2015, 27: 143-150.

Gobrogge K.L. Sex, drugs, and violence: neuromodulation of attachment and conflict in voles. Neuroscience of Aggression. Heidelberg: Springer; 2014. pp. 229-264.

Golden S.A., Covington H.E., Berton O., Russo S.J. A standardized protocol for repeated social defeat stress in mice. Nat Protoc. 2011, 6: 1183-1191.

Grafman J., Schwab K., Warden D., Pridgen A., Brown H.R., Salazar A.M. Frontal lobe injuries, violence and aggression: a report of the Vietnam Head Injury Study. Neurology. 1996, 46: 12311238.

Gregg T.R., Siegel A. Brain structures and neurotansmitters regulating aggression in cats: implications for human aggression. Prog Neuropsychopharmacol Biol Psychiatry. 2001, 25: 91-140.

Gutknecht L., Kriegebaum C., Waider J., Schmitt A., Lesch K.P. Spatiotemporal expression of tryptophan hydroxylase isoforms in murine and human brain: convergent data from Tph2 knockout mice. Eur Neuropsychopharmacol. 2009, 19: 266-282.

Gutknecht L., Popp S., Waider J., Sommerlandt F.M., Göppner C., Post A., Reif A., van den Hove D., Strekalova T., Schmitt A., Colaso M.B., Sommer C., Palme R., Lesch K.P. Interaction of brain 5HT synthesis deficiency, chronic stress and sex differentially impact emotional behavior in Tph2 knockout mice. kamarPsychopharmacology (Berl). 2015, 232: 2429-2441. 
Haller J., Horvath Z., Bakos N. The effect of buspirone on normal and hypoarousal-driven abnormal aggression in rats. Prog. Neuropsychopharmacol. Biol. Psychiatry. 2007, 31: 27-31.

Haller J., Kruk M.R. Normal and abnormal aggression: human disorders and novel laboratory models. Neurosci. Biobehav. 2006. 30: 292-303.

Haller J., van de Schraaf J., Kruk M.R. Deviant forms of aggression in glucocorticoid hyporeactive rats: a model for 'pathological' aggression? J Neuroendocrinol. 2001, 13: 102-107.

Herrmann N., Lanctôt K., Naranjo C. Behavioral disorders in demented elderly patients. Current issues in pharmacotherapy. CNS Drugs. 1996, 6: 280-300.

Hira S., Saleem U., Anwar F., Ahmad B. Antioxidants Attenuate Isolation- and L-DOPA-Induced Aggression in Mice. Front Pharmacol. 2017, 8: 945.

Hoerst M., Weber-Fahr W., Tunc-Skarka N., Ruf M., Bohus M., Schmahl C., Ende G. Correlation of Glutamate Levels in the Anterior Cingulate Cortex With Self-reported Impulsivity in Patients With Borderline Personality Disorder and Healthy Controls. Arch Gen Psychiatry. 2010, 67: 946-954.

Hong W., Kim D.W., Anderson D.G. Antagonistic Control of Social Behaviors by Inhibitory and Excitatory Neurons in the Medial Amygdala. Cell. 2014, 158: 1348-1361.

Jia N., Yang K., Sun Q., Cai Q., Li H., Cheng D., Fan X., Zhu Z. Prenatal stress causes dendritic atrophy of pyramidal neurons in hippocampal CA3 region by glutamate in offspring rats. Dev Neurobiol. 2010, 70: 114-125.

Kamarck T.W., Haskett R.F., Muldoon M., Flory J.D., Anderson B., Bies R., Pollock B., Manuck S.B. Citalopram intervention for hostility: results of a randomized clinical trial. J Consult Clin Psychol. 2009, 77: 174-188.

Kästner N., Richter H., Urbanik S., Kunert J., Waider J., Lesch K.P., Kaiser S., Sachser N. Brain serotonin deficiency affects female aggression. Sci Rep. 2019, 9: 1366.

Kiecolt-Glaser J.K., Loving T.J., Stowell J.R., Malarkey W.B., Lemeshow S., Dickinson S.L., Glaser R. Hostile marital interactions, proinflammatory cytokine production, and wound healing. Arch Gen Psychiatry. 2005, 62: 1377-1384.

Korn M.L., Plutchik R., Van Praag H.M. Panic-associated suicidal and aggressive ideation and behavior. J. Psychiat. Res. 1997, 31: 481487. 
Kostowski W., Cxlonkowski A., Markowdka L., Markiewicz L. Intraspecific aggressiveness after lesions of midbrain raphe nuclei in rats. Pharmacology. 1975, 13: 81-85.

Kudryavtseva N.N., Bondar N.P. Anxiolytic and anxiogenic effects of diazepam in male mice with different experience of aggression. Bull. Exp. Biol. Med. 2002, 133: 372-376.

Kulikov A.V., Gainetdinov R.R., Ponimaskin E., Kalueff A.V., Naumenko V.S., Popova N.K. Interplay between the key proteins of serotonin system in SSRI antidepressants efficacy. Expert Opin Ther Targets. 2018, 22: 319-330.

Kumar K.K., Tung S., Iqbal Bone loss in anorexia nervosa: Leptin, serotonin, and the sympathetic nervous system. Ann. N. Y. Acad. Sci. 2010, 1211: 51-65.

Landgraf R., Kessler M. S., Bunck M., Murgatroyd C., Spengler D., Zimbelmann M., Nussbaumer M., Czibere L., Turck C. W., Singewald N., Rujescu D., Frank E. Candidate genes of anxietyrelated behavior in $\mathrm{HAB} / \mathrm{LAB}$ rats and mice: focus on vasopressin and glyoxalase-I. Neurosci. Biobehav. Rev. 2007, 31: 89-102.

Lane S.D., Gowin J.L., Green C.E., Steinberg J.L., Moeller F.G., Cherek D.R. Acute topiramate differentially affects human aggressive responding at low vs. moderate doses in subjects with histories of substance abuse and antisocial behavior. Pharmacol Biochem Behav. 2009, 92: 357-362.

Lane S.D., Kjome K.L., Moeller G. Neuropsychiatry of Aggression. Neurol Clin. 2011, 29: 49.

Leroy F., Park J., Asok A., Brann D.H., Meira T., Boyle L.M., Buss E.W., Kandel E.R., Siegelbaum S.A. A circuit from hippocampal CA2 to lateral septum disinhibits social aggression. Nature. 2018, 564: 213-218.

Lesch K.P., Araragi N., Waider J., van den Hove D., Gutknecht L. Targeting brain serotonin synthesis: insights into neurodevelopmental disorders with long-term outcomes related to negative emotionality, aggression and antisocial behaviour. Philos Trans R Soc Lond B Biol Sci. 2012, 367: 2426-2443.

Lieving L.M., Cherek D.R., Lane S.D., Tcheremissine O.V., Nouvion S.O. Effects of acute tiagabine administration on aggressive responses of adult male parolees. J Psychopharmacol. 2008, 22: 144-152.

Llorca A. Malonda E., Samper P. The role of emotions in depression and aggression Med Oral Patol Oral Cir Bucal. 2016, 21: e559-e564. 
Lotrich F.E., Sears B., McNamara R.K. Anger induced by interferonalpha is moderated by ratio of arachidonic acid to omega- 3 fatty acids. J. Psychosom. Res. 2013, 75: 475-483.

Malone R.P., Bennett D.S., Luebbert J.F., Rowan A.B., Biesecker K.A., Blaney B.L., Delaney M.A. Aggression classification and treatment response. Psychopharmacol Bull. 1998, 34: 41-45.

Mann J.J., Brent D.A., Arango V. The neurobiology and genetics of suicide and attempted suicide: a focus on the serotonergic system. Neuropsychopharmacology. 2001, 24: 467-477.

Morie K.P., Garavan H., Bell R.P., De Sanctis P., Krakowski M.I., Foxe J.J. Intact inhibitory control processes in abstinent drug abusers (II): A high-density electrical mapping study in former cocaine and heroin addicts. Neuropharmacology. 2014, 82: 151-160.

Marino M.D., Bourdelat-Parks B.N., Cameron Liles L., Weinshenker D. Genetic reduction of noradrenergic function alters social memory and reduces aggression in mice. Behav. Brain Res. 2005, 161: 197-203.

Martone J. Can taming inflammation help reduce aggression? Current Psychiatry. 2019, 18: 49-50.

Masood A., Nadeem A., Mustafa S.J., O'Donnell J.M. Reversal of oxidative stress-induced anxiety by inhibition of phosphodiesterase-2 in mice. J Pharmacol Exp Ther. 2008, 326: 369-379.

McEwen B.S. Physiology and neurobiology of stress and adaptation: central role of the brain. Physiological reviews. 2007, 87: 873904.

Mommersteeg P.M., Vermetten E., Kavelaars A., Geuze E., Heijnen C.J. Hostility is related to clusters of T-cell cytokines and chemokines in healthy men. Psychoneuroendocrinology. 2008, 33: 1041-1050.

Morales I., Guzmán-Martínez L., Cerda-Troncoso C., Farías G.A., Maccioni R.B. Neuroinflammation in the pathogenesis of Alzheimer's disease. A rational framework for the search of novel therapeutic approaches. Front Cell Neurosci. 2014, 8: 112.

Müller-Spahn F. Behavioral disturbances in dementia. Dialogues Clin Neurosci. 2003, 5: 49-59.

Natarajan D., de Vries H., Saaltink D. J., de Boer S. F., Koolhaas J. M. Delineation of violence from functional aggression in mice: an ethological approach. Behav. Genet. 2009, 39: 73-90.

Navarro J.F., De Castro V., Martín-López M. JNJ16259685, a selective mGlu1 antagonist, suppresses isolation-induced aggression in male mice. Eur. J. Pharmacol. 2008, 586; 217-220. 
Nelson R. J., Demas G. E., Huang P. L., Fishman M. C., Dawson V. L., Dawson T. M., Snyder S. H. (1995). Behavioural abnormalities in male mice lacking neuronal nitric oxide synthase. Nature 378 , 383-386.

Nelson R.J., Chiavegatto S. Molecular basis of aggression. Trends Neurosci. 2001, 24: 713-719.

Neumann I.D., Veenema A.H., Beiderbeck D.I. Aggression and Anxiety: Social Context and Neurobiological Links. Front Behav Neurosci. 2010, 4: 12.

Newman E.L., Chu A., Bahamón B., Takahashi A., DeBold J.F., Miczek K.A., 2012. NMDA Receptor Antagonism: Escalation of Aggressive Behavior in Alcohol-drinking Mice. Psychopharmacology (Berl). 224, 167-177.

Park M.J., Seo B.A., Lee B., Shin H.S., Kang M.K. Stress-induced changes in social dominance are scaled by AMPA-type glutamate receptor phosphorylation in the medial prefrontal cortex. Scientific Reports. 2018, 8: 15008.

Patel A., Siegel A., Zalcman S.S. Lack of aggression and anxiolytic-like behavior in TNF receptor (TNF-R1 and TNF-R2) deficient mice. Brain Behav. Immun. 2010, 24: 1276-1280.

Patki G., Atrooz F., Alkadhi I., Solanki N., Salim S. High aggression in rats is associated with elevated stress, anxiety-like behavior, and altered catecholamine content in the brain. Neurosci Lett. 2015, 584: 308-313.

Popova N.K. From gene to aggressive behavior: The role of brain serotonin. Neurosci. Behav. Physiol. 2008, 38: 471-475.

Raine A., Ishikawa S.S., Arce E., Lencz T., Knuth K.H., Bihrle S., LaCasse L., Colletti P. Hippocampal structural asymmetry in unsuccessful psychopaths. Biol Psychiatry. 2004, 55: 185-191.

Raine A., Lencz T., Bihrle S., LaCasse L., Colletti P. Reduced prefrontal gray volume and autonomic deficits in antisocial personality disorder. Arch Gen Psychiatry. 2000, 57: 119-127.

Ramamurthi B. Stereotactic operation in behaviour disorders. Amygdalotomy and hypothalamotomy. Acta Neurochir Suppl. 1988, 44: 152-157.

Rammal H., Bouayed J., Soulimani R. A direct relationship between aggressive behavior in the resident/intruder test and cell oxidative status in adult male mice. Eur J Pharmacol. 2010, 627): 173-176.

Reiss AJ.J., Roth J.A. Understanding and preventing violence. Vol. 1. National Academy Press; Washington, DC, US: 1993. p. 464. 
Rinne T., van den Brink W., Wouters L., van Dyck R. SSRI treatment of borderline personality disorder: a randomized, placebocontrolled clinical trial for female patients with borderline personality disorder. American Journal of Psychiatry. 2002, 159: 2048-2054.

Rodriguiz R. M., Chu R., Caron M. G., Wetsel W. C. (2004). Aberrant responses in social interaction of dopamine transporter knockout mice. Behav. Brain Res. 148, 185-19810.1016/S01664328(03)00187-6

Salzman C., Jeste D.V., Meyer R.E., Cohen-Mansfield J., Cummings J., Grossberg G.T., Jarvik L., Kraemer H.C., Lebowitz B.D., Maslow K., Pollock B.G., Raskind M., Schultz S.K., Wang P., Zito J.M., Zubenko G.S. Elderly patients with dementia-related symptoms of severe agitation and aggression: consensus statement on treatment options, clinical trials methodology, and policy. J Clin Psychiatry. 2008, 69: 889-898.

Saudou F., Amara D. A., Dierich A., LeMeur M., Ramboz S., Segu L., Buhot M. C., Hen R. (1994). Enhanced aggressive behavior in mice lacking 5-HT1B receptor. Science 265, 1875187810.1126/science.8091214

Schoenbaum G., Setlow B., Saddoris M.P., Gallagher M. Encoding predicted outcome and acquired value in orbitofrontal cortex during cue sampling depends upon input from basolateral amygdala. Neuron. 2003, 39: 855-867.

Scordalakes E.M., Rissman E.F. Aggression in male mice lacking functional estrogen receptor alpha. Behav. Neurosci. 2003, 117: $38-45$.

Scott L.N., Wright A.G.C., Beeney J.E., Lazarus S.A., Pilkonis P.A., Stepp S.D. Borderline personality disorder symptoms and aggression: A within-person process model. J Abnorm Psychol. 2017, 126: 429-440.

Shelton D. Emotional disorders in young offenders. J Nurs Scholarsh. 2001, 33: 259-263.

Siegel A., Brutus M. Neural substrates of aggression and rage in the cat. Progress in Psychobiology and Physiological Psychology. New York, NY, Academic Press. 1990: 135-233.

Sluyter F., Arseneault L., Moffitt T. E., Veenema A. H., de Boer S., Koolhaas J. M. Toward an animal model for antisocial behavior: parallels between mice and humans. Behav. Genet. 2003, 33: $563-574$.

Stanford M.S., Anderson N.E., Lake S.L., Baldridge R.M. Pharmacologic treatment of impulsive aggression with 
antiepileptic drugs. Curr Treat Options Neurol. 2009, 11: 383390.

Stanford M.S., Helfritz L.E., Conklin S.M., Villemarette-Pittman N.R., Greve K.W., Adams D., Houston R.J. A comparison of anticonvulsants in the treatment of impulsive aggression. Exp Clin Psychopharmacol. 2005, 13: 72-77.

Stuart G.W. Preventing and Managing Aggressive Behavior. Chapter 28, Intergenerational Transmission of Violence. 2013, p. 575

Suarez E.C., Lewis J.G., Kuhn C. The relation of aggression, hostility, and anger to lipopolysaccharide-stimulated tumor necrosis factor (TNF)-alpha by blood monocytes from normal men. Brain Behav Immun. 2002, 16: 675-684.

Swanson J.W., Holzer C.E., Ganju V.K., Jono R.T. Violence and psychiatric disorder in the community: evidence from the Epidemiologic Catchment Area surveys. Hosp Community Psychiatry. 1990, 41: 761-770.

Taft C.T., Creech S.K., Murphy C.M. Anger and aggression in PTSD. Curr Opin Psychol. 2017, 14: 67-71.

Takahashi A., Flanigan M.E., McEwen B.S., Russo S.J. Aggression, Social Stress, and the Immune System in Humans and Animal Models. Front Behav Neurosci. 2018, 12: 56.

Toth M., Halasz J., Mikics E., Barsy B., Haller J. Early social deprivation induces disturbed social communication and violent aggression in adulthood. Behav. Neurosci. 2008, 122: 849-854.

van Oortmerssen G.A., Bakker T.C.M. Artificial selection for short and long attack latencies in wild Mus musculus domesticus. Behav Genet. 1981, 11: 115-126.

Vakili V., Ziaee M., Zarifan A,, 2015. Aggression: Is that an issue for worrying? Iran J Public Health. 44(11), 1561-1562.

Van Praag H.M. Anxiety/aggression--driven depression. A paradigm of functionalization and verticalization of psychiatric diagnosis. Prog Neuropsychopharmacol Biol Psychiatry. 2001, 25: Botsis J.A., Soldatos C.R., Stefanis C.N. Suicide: Biopsychosocial Approaches. Amsterdam: Elsevier; 1997. 893-924.

Veenema A.H., Blume A., Niederle D., Buwalda B., Neumann I.D. Effects of early life stress on adult male aggression and hypothalamic vasopressin and serotonin. Eur J Neurosci. 2006, 4: $1711-1720$.

Vekovischeva O.Y., Aitta-Aho T., Echenko O., Kankaanpää A., Seppälä T., Honkanen A., Sprengel R., Korpi E.R. Reduced aggression in AMPA-type glutamate receptor GluR-A subunit-deficient mice. Genes Brain Behav. 2004, 3: 253-265. 
Vekovischeva O.Y., Aitta-aho T., Verbitskaya E., Sandnabba K., Korpi E.R. Acute effects of AMPA-type glutamate receptor antagonists on intermale social behavior in two mouse lines bidirectionally selected for offensive aggression. Pharmacol Biochem Behav. 2007, 87: 241-249.

Virkkunen M., Goldman D., Nielsen D.A., Linnoila M. Low brain serotonin turnover rate (low CSF 5-HIAA) and impulsive violence. Journal of Psychiatry and Neuroscience. 1995, 20: 27127 Neuroscience. Author manuscript; available in PMC 2014 Apr 16.

Vukhac K.L., Sankoorikal E.B., Wang Y. Dopamine D2L receptor- and age-related reduction in offensive aggression. Neuroreport. 2001, 12: 1035-1038.

Wall V.L., Fischer E.K., Bland S.T. Isolation rearing attenuates social interaction-induced expression of immediate early gene protein products in the medial prefrontal cortex of male and female rats. Physiol Behav. 2012, 107: 440-450.

Wang Y., He Z., Zhao C., Li L. Medial amygdala lesions modify aggressive behavior and immediate early gene expression in oxytocin and vasopressin neurons during intermale exposure. Behav Brain Res. 2013, 245: 42-49.

Weidner M.T., Lardenoije R., Eijssen L., Mogavero F., De Groodt L.P.M.T., Popp S., Palme R., Förstner K.U., Strekalova T., Steinbusch H.W.M., Schmitt-Böhrer A.G., Glennon J.C., Waider J., van den Hove D.L.A., Lesch K.P. Identification of Cholecystokinin by Genome-Wide Profiling as Potential Mediator of Serotonin-Dependent Behavioral Effects of Maternal Separation in the Amygdala. Front Neurosci. 2019, 13: 460.

Wersinger S.R., Ginns E.I., O'Carroll A.M., Lolait S.J., Young W.S. Vasopressin V1b receptor knockout reduces aggressive behavior in male mice. Mol. Psychiatry. 2002, 7: 975-984.

World Health Organization. Geneva, Switzerland: World Health Organization; 2007. World health organization expert committee on problems related to alcohol consumption.

Yang C.R., Bai Y.Y., Ruan C.S., Zhou H.F., Liu D., Wang X.F., Shen L.J., Zheng H.Y., Zhou X.F. Enhanced aggressive behaviour in a mouse model of depression. Neurotox Res. 2015, 27: 129-142. Neurol Clin. Author manuscript; available in PMC 2012 Feb 1.

Yang Y., Glenn A.L., Raine A. Brain abnormalities in antisocial individuals: implications for the law. Behav Sci Law. 2008, 26: 65-83. 


\section{Chapter 2}

\section{Thiamine and benfotiamine}

counteract ultrasound-induced aggression, normalize AMPA receptor expression and plasticity markers, and reduce oxidative stress in mice 


\section{Chapter 2. Thiamine and benfotiamine counteract ultrasound-induced aggression, normalize AMPA receptor expression and plasticity markers, and reduce oxidative stress in mice}

Anna Gorlova $a^{a, b, c, d^{*}}$, Dmitrii Pavlove,b,d,e*, Daniel C. Anthony ${ }^{\mathrm{f}}$, Evgeny Ponomarevg, Margaux Sambon ${ }^{b}$, Andrey Proshin ${ }^{\mathrm{h}}$, Igor Shafarevich $^{c}$, Diana Babaevskaya ${ }^{c}$, Klaus-Peter Lescha,c,i, Lucien Bettendorffb\#, Tatyana Strekalovaa,c,e\#

aDepartment of Neuroscience, Maastricht University, Universiteitssingel 40, NL 6229ER, Maastricht, Netherlands; bLaboratory of Neurophysiology, GIGA-Neurosciences, University of Liège, av Hippocrate 1, 13-4000, Liege, Belgium; cLaboratory of Psychiatric Neurobiology and Department of Normal Physiology, Institute of Molecular Medicine, Sechenov First Moscow State Medical University Trubetskaya street 8-2, 119991, Moscow, Russia; dDepartment of Biology, Lomonosov Moscow State University, Leninskie Gory1-12, 119991, Moscow, Russia; eInstitute of General Pathology and Pathophysiology, Baltiiskaya str, 8, Moscow, Russia, 125315, Moscow, Russia; fDepartment of Pharmacology, Oxford University, Mansfield Road, OX1 3QT, Oxford, United Kingdom; sSchool of Biomedical Sciences, Faculty of Medicine, The Chinese University of Hong Kong, Hong Kong; hResearch Institute of Normal Physiology, Baltiiskaya str, 8, Moscow, Russia, 125315, Moscow, Russia; iDivision of Molecular Psychiatry, Center of Mental Health, University of Würzburg, Josef-Schneider-Straße 2, 97080, Wuerzburg, Germany Würzburg, Germany

*Equal contribution 
\# Joint Corresponding authors

\section{Dr. Tatyana Strekalova}

Department of Neuroscience

Maastricht University

Universiteitssingel 40, NL 6229

ER Maastricht, The Netherlands

+314338 84110

Fax: +31433671096

E-mail: t.strekalova@maastrichtuniversity.nl
Prof. Lucien Bettendorff

GIGA-Neurosciences

University of Liege

av Hippocrate 1, 13-4000

Liege, Belgium Tel:

Tel: +32 93120177600

Fax: +32 93120177620

E-mail: 1.bettendorff@ulg.a.be

\section{Abstract}

The negative societal impacts associated with the increasing prevalence of violence and aggression is increasing, and, with this rise, is the need to understand the molecular changes that underpin stress-induced aggressive behavior. In mice, stressinduced aggression is known to alter AMPA receptor subunit expression, plasticity markers, and oxidative stress within the brain. Here, we induced aggression in BALB/c mice using chronic ultrasound exposure as a model of "emotional" stress and examined the impact of the psychoactive anti-oxidant compounds thiamine (vitamin B1), and its derivative benfotiamine, on AMPA receptor subunit expression, established plasticity markers, and oxidative stress. The administration of thiamine or benfotiamine (200 mg/kg/day) in drinking water decreased aggressive behavior following 3-weeks of ultrasound exposure and benfotiamine, reduced floating behavior in the swim test. The vehicle-treated 
ultrasound-exposed mice exhibited increases in protein carbonyl and total glutathione, altered AMPA receptor subunits expression, and decreased expression of plasticity markers. These ultrasound-induced effects were ameliorated by thiamine and benfotiamine treatment; in particular both antioxidants were able to reverse ultrasound-induced changes in GluA1 and GluA2 subunit expression, and, within the prefrontal cortex, significantly reversed the changes in protein carbonyl and polysialylated form of neural cell adhesion molecule (PSA-NCAM) expression levels. Benfotiamine was usually more efficacious than thiamine. Thus, the thiamine compounds were able to counteract ultrasound-induced aggression, which was accompanied by the normalization of markers that have been showed to be associated with ultrasound-induced aggression. These commonly used, orally-active compounds may have considerable potential for use in the control of aggression within the community.

Keywords: Aggression, emotional stress, brain oxidative stress, plasticity, thiamine, mice.

\section{Introduction}

Both genetic and environmental factors have been shown to regulate aggressive traits via complex codependent mechanisms 
(Caspi et al. 2002; Lesch et al., 2012), but a causal role for environmental stress is well documented (Angkaw et al., 2013; Fanning et al., 2015, 2017) and is associated with overt aggression in humans (Kim and Haller, 2007; Kessler et al., 2015). Experimental models have also provided support for this relationship (Veenema and Neumann, 2007; Rammal et al., 2010; Costa-Nunes et al., 2014; Malki et al., 2016). In particular, emotional stress, which is referred to as state that is primarily triggered by the perception and cognitive evaluation of adverse events rather than a disturbance of physical nature (Chrouses and Gold, 1992; Fontes et al., 2014), appears to be the type of stress that most frequently results in overt aggressiveness (Angkaw et al., 2013; Fanning et al., 2015, 2017). We have shown that we can induce behavioral changes that are consistent with emotional stress, including aggression and, at a molecular level, oxidative stress within the hippocampus, using chronic ultrasound exposure (Pavlov et al., 2019). However, hitherto our investigation of emotional-stress-induce aggression has been limited to a small number of outcome measures and we have not sought to target the stress-induced molecular changes such as the use of therapy that reduces oxidative stress. Under stress paradigms have also been shown impact on plasticity markers, but, prior to this study, it was unclear how ultrasound exposure would affect plasticity markers and whether changes would be amenable to therapy. Plasticity markers that have been linked to the regulation of social behaviour and emotionality include 
postsynaptic density protein 95 (PSD95) (Been et al., 2016), polysialylated form of neural cell adhesion molecule (PSA-NCAM) (Angata et al., 2004; Winkler et al., 2017), and B-catenin (Maguschak and Ressler, 2012).

Hans Selye defined stress as "the non-specific response of the body to any demand for change" (Selye, 1974), which, in case of emotional stress, is accompanied by physiological responses to any stimulus of a potentially threatening nature (Simonov, 1997; Clark et al., 2009). The use of the term "stress" extends to other fields of research and now includes stress at a cellular level. For instance, excessive formation of free radicals in mitochondria is described as a form of cellular stress known as "oxidative stress". Increased oxidative stress within the brain can play a key role in the pathophysiology of emotional stress and in the accompanying behavioural abnormalities, including excessive aggression (Boufleur et al., 2013; Okazawa et al., 2014; Schiavone et al., 2015; Cline et al., 2015a; Patki et al., 2013, 2014; Fanning et al., 2015). Mitochondrial dysregulation and microglia activation are associated with oxidative stress, and can lead to neuronal dysfunction, compromised brain plasticity, and abnormal emotionality (Block and Hong, 2007; Roy et al., 2008; Marais et al., 2009; Diehl et al., 2012; Boufleur et al., 2013). Oxidative stress markers have been found to be elevated in neuropsychiatric conditions, which are often accompanied by inappropriate aggression, such as autism or attention deficit/hyperactivity disorder (Ng et al., 2008; Gvozdjáková et al., 2014), panic disorder 
(Kuloglu et al., 2002), aging-related neurodegeneration (Okazawa et al., 2014; Salminen et al., 2014), and certain forms of affective abnormalities and personality disorders (Fanning et al., 2015; Coccaro et al., 2013; 2016; Schiavone et al., 2015). For example, elevated plasma levels of 8-hydroxy-2'-deoxyguanosine was found in patients with intermittent explosive disorder and correlated with incidence of aggressive episodes both in healthy and affected individuals (Coccaro et al., 2016).

A wide range of therapies that ameliorate oxidative stress have been tested for their potential to improve signs of excessive aggression; for instance, in aggressive young adults (Hamazaki et al., 1996), in patients with autism (Gvozdjáková et al., 2014, Stutzman and Dopheide, 2015), in mice subjected to stress of group housing (Strekalova et al., 2018), and in rats exposed to the stress of sleep deprivation (Solanki et al., 2016). Heavy metal exposure is also known to induce aggression and amenable to therapy after exposure to toxic concentrations of lead (Ebuehi et al., 2012), and cadmium (El-Tarras et al., 2016). Thiamine (vitamin B1) and its derivatives are regulators of mitochondrial function and metabolism (Bettendorff et al, 2014) and are known to exert anti-oxidative and anti-stress effects in in vitro models (Schmid et al., 2008; Bozic et al., 2015; Raj et al., 2018) and in experimental models of stress (Markova et al., 2017; Vignisse et al., 2017; Pavlov et al., 2017), traumatic brain injury (Mkrtchyan et al., 2018) and neurodegeneration (Pan et al., 2010, 2016; Tapias et al., 2018). We have recently shown that a two-week pre- 
treatment regime in mice with thiamine, or its more bioavailable analogue benfotiamine can prevent increases in the GSK3-beta activity and measures of depressive-like and anxiety-like behavior following both predator stress and modified swim test paradigms (Markova et al., 2017; Vignisse et al., 2017; Pavlov et al., 2017). The compounds also rescued hippocampal neurogenesis and protein carbonyl levels after predation stress (Vignisse et al., 2017). Other work has revealed the beneficial effects of benfotiamine on amyloid pathology (Pan et al., 2010, 2016) and tau pathology mediated via Nrf/2 antioxidant response element (ARE)-dependent gene expression (Tapias et al., 2018). Hitherto, it was unclear how thiamine and benfotiamine might affect aggression in animal models.

A number of neurotransmitter systems and hormones have been linked to aggression including glutamate. Increased glutamatergic tone leads to augmentation of aggressiveness in many species (Bullock and Rogers 1986; Munoz-Blanco et al. 1986). In particular, dysregulation of AMPA receptors is likely to play an important role in stress-induced aggression (Arnstem, 2009; Coccaro et al., 2013; Strekalova et al., 2018; Khlghatyan et al., 2018) as Y-carboline compounds, designed to target AMPA receptors and 5-HT6 receptors, have been found to have antiaggressive effects in social stress paradigms in mice (Strekalova et al., 2018). Furthermore, altered gene expression of AMPA receptor subunit GluA2 and 5-HT6 receptors in the brain was found in stressed mice in a heterozygous tryptophan hydroxylase 
2 (TPH2) knockout (Bazhenova et al., 2017). Gene association studies have also suggested a link between the 5-HTR6 gene functions and aggression during depression (Azenha et al., 2009), schizophrenia (Tsai et al., 1999) and neurodegeneration (Marcos et al., 2016). AMPA-type glutamate receptors are tetramers composed of subunits GluA1-4 and they mediate a much of the fast synaptic neurotransmission that is implicated in neuroplastic processes. GluA1, GluA2, GluA3, and GluA4 can be assembled in either homomers or heteromers (Hollmann et al. 1989; Keinänen et al. 1990) and they are widely expressed throughout the brain on neurons and glia with the exception of GluA4 that is expressed at a very low level outside the cerebellum. The subcellular localization of AMPA receptors indicates that $60-70 \%$ of the receptors are at intracellular sites, thus forming a reserve pool of receptors unless they possess an unknown duty distinct from the silent synapse theory (Lee et al. 2001).

Aggressive behaviour has been explored in the AMPA subunit mutants; mice lacking GluA1 exhibit reduced anxiety and male aggression (Adamczyk, et al., 2012), GluA2 knock out animals are less aggressive than wild-type controls (Shimshek et al., 2006), and the GluA3 deficient mouse was found to exhibit increased aggressive behaviour (Adamczyk, et al., 2012). Furthermore, the administration of AMPA receptor antagonists to Turku Aggressive mice has been shown to decrease the biting component of their aggressive behaviour. However, it is clear that 
aggressive traits are associated with regional variations in AMPA receptor expression. In the amygdala, four weeks of social isolation increased AMPA receptor subunits GluA1 and GluA2, but isolation rearing increases the expression of AMPA receptor subunits (GluA1, GluA2 and GluA3) in the prefrontal cortex, but not in the hippocampus or nucleus accumbens (Araki et al., 2014). The prefrontal cortex is known to regulate social behaviour and aggressive responses (Siever, 2008; Machado and Bachevalier, 2006), there is less understanding of the molecular changes that underpin decision to engage in aggression. Reactive aggression, in particular, is associated with altered prefrontal activity where AMPA receptor expression is high, but, hitherto, it was unclear how aggression would affect AMPA subunit expression in the prefrontal cortex in a model of emotional stress. The expression of the AMPA receptors in rodents is also high in the hippocampal subregions CA1, CA3 and dentate gyrus, which is also know to play an important role in deciding whether to attack (Leroy et al., 2018) based on evaluation of memories, including memories of past social interactions. Given these observations, we were interested to discover whether aggression induced in a model of emotional stress would affect the expression of AMPA subunits and 5HT-6 receptor and what would be the effect of the psychoactive antioxidants.

Mice that are subject to recently established model of emotional stress, in which "emotionally negative" and "neutral" randomly alternating frequencies of ultrasound with the range 
20-45 kHz (Constantini and D'Amato, 2006) for three weeks display increased aggressive and depressive-like behaviors, decreased hippocampal neurogenesis (Morosova et al., 2016; Pavlov et al., 2019). Here, we sought to investigate what impact chronic ultrasound exposure would have on the expression brain oxidative stress markers, plasticity markers, and AMPA subunit receptor expression. Furthermore, we sought to discover whether the administration of thiamine or its derivative, benfotiamine would normalize ultrasound-induced aggression and other emotional behaviors and whether their administration would ameliorate the molecular changes. Increases in aggression in the mice were accompanied, at a molecular level, by profound changes in markers of oxidative stress, AMPA subunit expression, and in key pathways that regulate emotionality and neural plasticity, including postsynaptic density protein 95 (PSD95), polysialylated form of neural cell adhesion molecule (PSA-NCAM) and B-catenin. These changes were largely reversed by the administration of thiamine or benfotiamine.

\section{Materials and methods}

\subsection{Animals and housing conditions}

The study was performed using BALB/c 10-12-week-old male mice that were obtained from certified Charles River provider, Stolbovaja, RAS, Moscow region (http://www.spfanimals.ru/about/providers/animals). Mice were housed individually in the standard cages $(27 \times 22 \times 15)$, under controlled 
laboratory conditions $\left(22 \pm 1^{\circ} \mathrm{C}, 55 \%\right.$ humidity) and maintained on a reversed 12-hour light/dark cycle (lights on at 19:00), with food and water provided ad libitum. All mice have been tested during the dark phase of light/dark cycle. Laboratory housing conditions and experimental procedures were set up and maintained in accordance with Directive 2010/63/EU of 22 September 2010 and carried out under the approval of the local veterinarian committee (10-02-15N72). All efforts were undertaken to minimize the potential discomfort of animals during the study.

\subsection{Study design}

In both chronic and acute ultrasound exposure experiments, mice were weighed and assigned to control and ultrasoundexposed groups (data not shown); ultrasound exposure was applied as described elsewhere (Morozova et al., 2016, Strekalova et al., 2018; Pavlov et al., 2019). Mice were subjected to a 3 -week exposure of ultrasound of unpredictably alternating frequencies (see below), and after behavioural testing, were additionally investigated for brain levels of oxidative stress markers, carbonyl protein and total glutathione, gene and protein expression of AMPA receptor subunits GluA1-4, 5-HT6 receptor, and protein expression of PSD95, PSA-NCAM and B-catenin using RT-PCR and Western blot (see below; Fig.1A). Subgroups of mice from the ultrasound-exposed group were treated with thiamine or benfotiamine at the dose of $200 \mathrm{mg} / \mathrm{kg} /$ day via drinking water, as described elsewhere (Markova et al., 2017; Vignisse et al., 2017; Pavlov et al., 2017). Mouse behavior was investigated twenty-four 
hours after the termination of ultrasound exposure in a novel cage, a social interaction test and in the forced swim test; they were killed sixteen hours after the behavioural testing, their brains were dissected (see below; Fig 1A).

To control for possible acute effects of ultrasound exposure on behavior, brain oxidative markers and molecular changes, and to rule out the possibility of acute effects of thiamine and benfotiamine, we performed a study with an acute $24 \mathrm{~h}$ ultrasound exposure experiment and administration of thiamine compounds. Mice were treated with either compound used at the dose of 200 $\mathrm{mg} / \mathrm{kg} /$ day via drinking water starting at the onset of ultrasound exposure; $24 \mathrm{~h}$-fluid intake was measured to control drug intake with drinking liquid. After acute $24 \mathrm{~h}$ exposure to the ultrasound exposure, animals were behaviourally studied in the novel cage, a social interaction test and a forced swim test, and they were culled $16 \mathrm{~h}$ afterwards and their brains were dissected (see below; Fig.1B).

Behavioural testing was performed as described elsewhere (Vignisse et al., 2017; Strekalova et al., 2018; Pavlov et al., 2019). Control mice were housed under similar conditions, but not exposed to the ultrasound radiation and studied simultaneously with ultrasound-exposed animals. Animals from the control group were littermates of mice from experimental groups, they were housed in an adjacent (separate) room, in order to eliminate the potential effects of stray ultrasound radiation. All manipulations on control and experimental animals were performed 
simultaneously to rule out possible confounding effects of seasonal inter-batch and other differences. On average, 7-8 animals per group were used; group sizes are indicated in figure legends.

\subsection{Ultrasound radiation}

Ultrasound exposure was performed as described elsewhere (Morozova et al., 2016; Strekalova et al., 2018; Pavlov et al., 2019). For a 3 -week or a $24 \mathrm{~h}$ period, ultrasound radiation of average intensity of $50 \pm 5 \mathrm{~dB}$ and variable frequencies in a 20 $45 \mathrm{~Hz}$ range was constantly delivered within a laboratory environment to experimental groups of mice using a random schedule of alternating frequencies via a commercially available device (Weitech, Wavre, Belgium). The range of ultrasound stimulation frequency was alternated every 10 minutes between frequencies $20-25 \mathrm{kHz}, 25-40 \mathrm{kHz}$ and $40-45 \mathrm{kHz}$; the distribution of the ultrasound radiation was controlled by the ultrasound detector (Discovery Channel, Rochester, NY, USA). The shape of the ultrasound signal was fluctuating, thus, mimicking natural ultrasonic vocalizations of mice (Constantini and D'Amato, 2006). Further details on the ultrasound radiation can be found in the Supplementary material (Supplementary material, Pavlov et al., 2019). The selectivity of the adverse effects of low-frequency ultrasound during the radiation period versus the potential general negative effects of a constant noise accompanying the procedure described here was demonstrated previously (Morozova et al., 2016). As a part of these experiments, when 
mixed frequencies ("white noise") at the range of $16-20 \mathrm{kHz}$ were employed, no changes in social and depressive-like behaviours were observed. In addition, it was demonstrated that a shortened period of ultrasound exposure of 1 to 2 -weeks duration were ineffective in producing behavioral changes and increase of serum corticosterone level, as compared with a 3-weeks exposure (Morozova et al., 2016; Pavlov et al., 2019).

Additional validation studies were performed in BALB/c mice that were exposed to different ranges of ultrasound frequency to understand whether the low frequency radiation is responsible for the behavioural changes (Supplemental Fig.1). $\mathrm{BALB} / \mathrm{c}$ mice were also exposed for 1,2 or 3 -weeks ultrasound treatment and the levels of corticosterone and changes in depressive-like behaviours were assessed to determine the optimum exposure time required to induce a depression-like phenotype (Supplemental Fig.2). Further validation studies showed that applied here 3-week ultrasound exposure has evoked anxiety-like behavior in the elevated maze in BALB/c mice (Supplemental Fig.3). Anxiogenic-like changes in the elevated plus maze, open field, depressive-like behaviours in the tail suspension test and increases in the measures of aggressive behavior were precluded by anxiolytic and antidepressant treatment with buspirone (Supplemental Fig.3). Finally, CBA male mice exposed to the 3-week ultrasound procedure displayed elevated aggressive behavior in the social interaction and social exploration tests (Supplemental Fig.4). These changes were 
accompanied by increased blood corticosterone levels that were not found after 1- or 2-week of ultrasound exposure (Supplemental Fig.4). Thus, additional validation studies of the 3-week ultrasound exposure evidence its profound negative effects on the emotional behaviors and blood corticosterone levels of mice.

\subsection{Behavioral Tests}

\subsubsection{Social interaction test}

A social interaction test was employed here and was an adaptation of a previously described methodology (Costa-Nunes, 2014; Couch et al., 2016; Strekalova et al., 2018). Mice from the experimental group were placed individually in an observation cage $(30 \times 60 \times 30 \mathrm{~cm})$ for $30 \mathrm{~min}$ to acclimatize. Thereafter, a previously group-housed naïve male mouse of the same strain and a similar weight and age, was introduced and left with the experimental mouse for $8 \mathrm{~min}$. During this period, mice were scored for the latency, total duration and number of episodes of following measures of aggressive and dominant-like behaviours: attacks, crawl-over behaviour, following behavior, and the incidence of tail rattling behavior was also scored. Additionally, these three parameters were measured for two forms of neutral social exploration: nose-nose and nose-anus contacts. 


\begin{tabular}{|c|c|c|c|c|c|c|c|c|}
\hline \multirow{3}{*}{$\begin{array}{c}\text { Days 1-21 } \\
\text { Ultrasound } \\
\text { exposure } \\
\text { Treatment with } \\
\text { thiamine } \\
\text { or benfotiamine } \\
\text { BALB/c mice }\end{array}$} & \multirow[t]{3}{*}{$\stackrel{+24 h}{\rightarrow}$} & \multirow{3}{*}{\begin{tabular}{|c} 
Day 22 \\
Behavioral \\
Testing
\end{tabular}} & \multirow[t]{3}{*}{$\stackrel{+16 h}{\rightarrow}$} & \multirow{3}{*}{$\begin{array}{c}\text { Day } 23 \\
\text { Tissue } \\
\text { Collection }\end{array}$} & \multirow{3}{*}{$\begin{array}{l}> \\
\searrow\end{array}$} & $\begin{array}{l}\text { Amygdala, } \\
\text { striatum, } \\
\text { raphe dorsalis }\end{array}$ & \multirow{3}{*}{$\begin{array}{l}\rightarrow \\
\rightarrow \\
\rightarrow\end{array}$} & \multirow{3}{*}{\begin{tabular}{|c} 
Protein \\
carbonyl, total \\
glutathione \\
assays
\end{tabular}} \\
\hline & & & & & & & & \\
\hline & & & & & & $\begin{array}{l}\text { Hippocampus, } \\
\text { prefrontal cortex }\end{array}$ & & \\
\hline
\end{tabular}

B

ACUTE ULTRASOUND EXPOSURE

\begin{tabular}{|c|c|c|c|c|c|c|c|c|}
\hline $\begin{array}{c}\text { Day } 1 \\
\text { Ultrasound } \\
\text { exposure } \\
\text { Treatment with } \\
\text { thiamine } \\
\text { or benfotiamine } \\
\text { BALB/c mice }\end{array}$ & $\stackrel{+24 \mathrm{~h}}{\rightarrow}$ & $\begin{array}{c}\text { Day } 2 \\
\\
\text { Behavioral } \\
\text { Testing }\end{array}$ & $\stackrel{+16 h}{\rightarrow}$ & $\begin{array}{c}\text { Day } 3 \\
\\
\text { Tissue } \\
\text { Collection }\end{array}$ & $\rightarrow$ & $\begin{array}{c}\text { Hippocampus, } \\
\text { prefrontal cortex }\end{array}$ & $\rightarrow$ & $\begin{array}{c}\text { Protein } \\
\text { carbonyl, } \\
\text { qRT-PCR, } \\
\text { Western blot } \\
\text { assays }\end{array}$ \\
\hline
\end{tabular}

Figure 1. Experiment design. After (A) 21-day- (chronic) or (B) 24h- (acute) exposure of ultrasound exposure and concomitant dosing with thiamine or benfotiamine, both control (no stress) and stress (ultrasound exposure) groups were tested in a battery of behavioral tests, brains were dissected for a subsequent oxidative stress assay, qRT-PCR and Western blot study. qRT-PCR - quantitative reverse transcription polymerase chain reaction assay. 


\subsubsection{Forced swim test}

This test was carried out as described previously (Malatynska et al., 2012; Strekalova et al., 2015; Pavlov et al., 2017). Mice were placed into a plastic transparent pool (diameter of $15 \mathrm{~cm}$ and a height of $25 \mathrm{~cm}$ ) filled with water to the depth for $17 \mathrm{~cm}$; water temperature was $+23^{\circ} \mathrm{C}$, light intensity was $15 \mathrm{Lux}$. Total duration of floating behavior, defined by the absence of any directed movements of animals' head and body, was scored offline during the 6 min-period at three 2-min intervals using Any-maze software (Stoelting Co, Wood Dale, IL, USA). Latency to float was evaluated as well.

\subsubsection{Locomotor behaviour}

Vertical exploratory activity of mice was studied in the novel cage test under red light as described elsewhere (Strekalova and Steinbusch, 2010; Couch et al., 2013). Mice were placed into a plastic cage, their number of exploratory rears was counted for a 5-min under red light. Additionally, total distance travelled was scored to study their horizontal activity over this time period.

\subsection{Brain Collection}

Mice were terminally anaesthetized with an intraperitoneal injection of Nembutal (Bayer, Wiesbaden, Germany) and then were transcardially perfused with $10 \mathrm{ml}$ of the ice-cold $0.9 \% \mathrm{NaCl}$. Their brains were dissected, and prefrontal cortex, hippocampus, amygdala, dorsal raphe and striatum were isolated for measurement of oxidative stress markers: two former structures 
were used for gene and protein expression studies (Fig.1A). All samples were immediately frozen at $-80^{\circ} \mathrm{C}$ on a dry ice.

2.6. Protein carbonyl assay

Determination of protein carbonyls using the OxiSelect ${ }^{\mathrm{TM}}$ Protein Carbonyl Fluorometric Assay kit (Cell Biolabs, Inc., San Diego, USA). After dissection, brain tissue was stored at $-80^{\circ} \mathrm{C}$ until use. Glass-glass homogenization followed by a sonification was performed on ice in $1 \mathrm{ml}$ of $1 \times$ Sample Diluent from the OxiSelect ${ }^{\mathrm{TM}}$ kit, centrifuged at $10000 \mathrm{~g}$ for $5 \mathrm{~min}$ at $4^{\circ} \mathrm{C}$, and the supernatant was removed. The total protein concentration was adjusted to $1-10 \mathrm{mg} / \mathrm{ml}$ with $1 \times$ Sample Diluent and protein carbonyls were determined according to the guidelines of the manufacturer using the GloMax Multi Detection System (Promega, Madison, WI, USA) equipped with a fluorescence module (485/540 $\mathrm{nm}$ filter set). Results were normalized to protein concentration as described previously (Vignisse et al., 2017; Pavlov et al., 2019).

\subsection{Total glutathione assay}

Oxidative stress was also estimated by the determination of total glutathione contents using the BioVision ${ }^{\mathrm{TM}}$ Glutathione Fluorometric Assay Kit (BioVision, Inc., San Francisco, USA) as described elsewhere (Pavlov et al., 2019). In total, $40 \mathrm{mg}$ of each sample was homogenized by glass-glass homogenization followed by a sonification on ice with $100 \mathrm{\mu l}$ of ice cold Glutathione Assay Buffer and total glutathione contents were determined according to the guidelines of the manufacturer using the GloMax Multi 
Detection System (Promega, Madison, WI, USA) equipped with a fluorescence module (340/420 nm filter set).

2.8. Quantitative real-time PCR

Quantitative real-time PCR was carried out as described elsewhere (Couch et al., 2013, 2016; Pavlov et al., 2017). Briefly, $1 \mathrm{\mu g}$ total RNA was converted into cDNA using random primers and Superscript III transcriptase (Invitrogen, Carlsbad, CA, USA). qRT-PCR was performed using the SYBR Green master mix (Bio-Rad Laboratories, Philadelphia, PA, USA) and the CFX96 Deep Well Real-Time PCR Detection System (Bio-Rad, Hercules, CA, USA). Further details in the protocol used and sequences of primers can be found in Supplementary material and Supplementary Table 1. The housekeeping gene glyceraldehyde3-phosphate dehydrogenase $(G A P D H)$ was selected as the reference gene since previous experiments demonstrated relatively low variability in GAPDH expression in the limbic structures of the rodents exposed to the ultrasound exposure (Morozova et al., 2016; Pavlov et al., 2019). Data were normalized to $G A P D H$ mRNA expression and calculated as relative-fold changes compared to control mice as described elsewhere (Morozova et al., 2016; Couch et al., 2016; Pavlov et al., 2017, 2019).

2.9. Protein isolation and Western blot analysis

Tissue samples were prepared using glass-glass homogenization followed by a sonification in ice-cold buffer (Roche Diagnostics, Indianapolis, IN, USA). Details of the 
Western blot analysis can be found in the Supplementary material and Supplementary Table 2. Briefly, protein concentration was quantified using the BCA protein assay kit (Pierce, Rockford, IL, USA, see Supplementary material), $25 \mu \mathrm{g}$ of proteins were treated with SDS-PAGE and electroblotted on the PVDF membrane (EMD Millipore, Billerica, MA, USA). These samples were incubated with primary antibodies overnight at $4^{\circ} \mathrm{C}$ (for information on antibodies used, see Supplementary material) that was followed by the incubation with respective secondary antibodies (Sigma-Aldrich, St. Louis, MO, USA) for $2 \mathrm{~h}$ at room temperature. Immunoreactive bands were detected using BioRad Molecular Imager Software (BioRad Laboratories, Richmond, CA, USA). B-tubulin was used as a reference protein as low fluctuation of its expression was found previously in similar experiments (Morozova et al., 2016; Pavlov et al., unpublished data); expression of proteins of interest were calculated in fold changes from levels of B-tubulin, as described elsewhere (Morozova et al., 2016; Pavlov et al., 2019).

\subsection{Drug administration}

Experimental solutions replaced normal drinking water. Thiamine or benfotiamine (Sigma-Aldrich, St. Louis, MO, USA) were dissolved in tap water and were changed every 4-5 days as described elsewhere (Markova al., 2017; Vignisse et al., 2017). The solutions of benfotiamine and thiamine were adjusted to $\mathrm{pH}$ 7.0, and drinking behaviour of mice was monitored by evaluating the 24-h liquid intake during the first three days of dosing during 
chronic experiment and at the end of a 24 -h period with acute ultrasound exposure study. We found no group differences in fluid intake suggesting normal drinking behavior in ultrasoundexposed and dosed mice (see Supplemental material, Supplemental Fig.5). The route and choice of dose was based on previous studies showing that no significant changes in weekly measured total liquid intake is observed during the ultrasound exposure (see Supplemental material, Supplemental Fig.5) (Markova al., 2017, Vignisse et al., 2017; Pavlov et al., 2017, 2019).

\subsection{Statistical analysis}

Data analysis was performed using GraphPad Prism software version 5.03 for Windows (San Diego CA, USA). Data were treated by a two-way ANOVA test; Tukey's test was used for following post-hoc analysis. Statistical significance was set at $\mathrm{p}<0.05$. Data are shown as mean \pm SEM.

\section{Results}

3.1. Ultrasound exposure induces aggressive and depressivelike behavioral changes in mice

In the social interaction test, a two-way ANOVA revealed a significant ultrasound $\mathrm{x}$ treatment interaction in latency to attack $\left(\mathrm{F}_{2,37}=3.258, \mathrm{p}=0.0498\right)$, total number of attacks $\left(\mathrm{F}_{2,37}=8.912\right.$, $\mathrm{p}=0.0007)$, total duration of attacks $\left(\mathrm{F}_{2,37}=11.12\right.$, $\mathrm{p}=0.0002$, twoway ANOVA) and total duration of crawl-over behavior $\left(\mathrm{F}_{2,37}=6.359, \mathrm{p}=0.0042\right.$ two-way ANOVA). More specifically, 
ANOVA revealed significant ultrasound effect for total number of attacks $\left(\mathrm{F}_{1,37}=6.087, \mathrm{p}=0.0185\right)$, total duration of attacks $\left(\mathrm{F}_{1,37}=4.808, \mathrm{p}=0.0347\right.$, two-way ANOVA $)$ and total duration of crawl-over behavior $\left(\mathrm{F}_{1,37}=4.306, \mathrm{p}=0.045\right.$ two-way ANOVA; Fig.2A), indicating increased ultrasound-induced aggressiveness in the treated mice. Furthermore, ANOVA revealed significant treatment effect for latency to attack $\left(\mathrm{F}_{2,37}=8.248, \mathrm{p}=0.0011\right)$, total number of attacks $\left(\mathrm{F}_{2,37}=17.25, \mathrm{p}<0.0001\right)$, total duration of attacks $\left(\mathrm{F}_{2,37}=18.57, \mathrm{p}<0.0001\right.$, two-way ANOVA $)$ and total duration of crawl-over behavior $\left(\mathrm{F}_{2,37}=7.992, \mathrm{p}=0.0013\right.$ two-way ANOVA; Fig.2A), which was supportive of an anti-aggressive effect of the psychoactive antioxidant drugs thiamine and benfotiamine.

Post-hoc analysis revealed significant increase of latency to attack in ultrasound-exposed group treated with thiamine ( $p=0.0413$, Tukey's test) or benfotiamine ( $p=0.0022$, Tukey's test; Fig.2A) in comparison with ultrasound-exposed non-treated group. It revealed significant increase of number of attacks in ultrasound-exposed non-treated group in comparison with naïve mice ( $p=0.0002$, Tukey's test) and a significant decrease of this parameter in ultrasound-exposed group treated with thiamine $(p<0.0001$, Tukey's test) or benfotiamine ( $<<0.0001$, Tukey's test; Fig.2A) in comparison with ultrasound-exposed non-treated group. Post hoc analysis also revealed significant increase of total duration of attacks in ultrasound-exposed non-treated group in comparison with naïve mice $(p<0.0001$, Tukey's test) and a 
significant decrease of this parameter in ultrasound-exposed group treated with thiamine $(\mathrm{p}<0.0001$, Tukey's test $)$ or benfotiamine ( $p<0.0001$, Tukey's test; Fig.2A) in comparison with ultrasound-exposed non-treated group. Finally, it revealed significant increase of total duration of crawl-over behavior in ultrasound-exposed non-treated group in comparison with naïve mice ( $p=0.0020$, Tukey's test) and a significant decrease of this parameter in ultrasound-exposed group treated with thiamine ( $p=0.0020$, Tukey's test) or benfotiamine $(p<0.0013$, Tukey's test; Fig.2A).

Additional analysis of aggressive-like behavior in the social interaction test revealed a significant decrease in latency to crawl-over, total number and total duration of crawl-over behavior and a significant increase in number of followings in vehicle-treated mice $(\mathrm{p}<0.05$ vs.control, two-way ANOVA and post hoc Tukey's test, see Supplementary material; Supplemental Fig. 6A,B). Both ultrasound-exposed thiamine-treated and benfotiamine-treated animals exhibited no change from control mice in these parameters and displayed significant differences from the ultrasound-exposed vehicle-treated group $(\# p<0.05$ vs. ultrasound-exposed group, two-way ANOVA and post hoc Tukey's test). There were no significant changes between groups in latency to interact and total duration of followings as well as in latency to tail rattling, number of tail rattling and total duration of tail rattling ( $p>0.05$ vs. ultrasound-exposed group, two-way 
ANOVA and post hoc Tukey's test, see Supplementary material; Supplemental Fig. 6C).

\section{3-WEEK ULTRASOUND EXPOSURE}

A

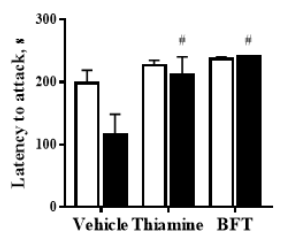

B

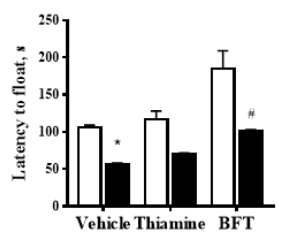

$\mathbf{E}$
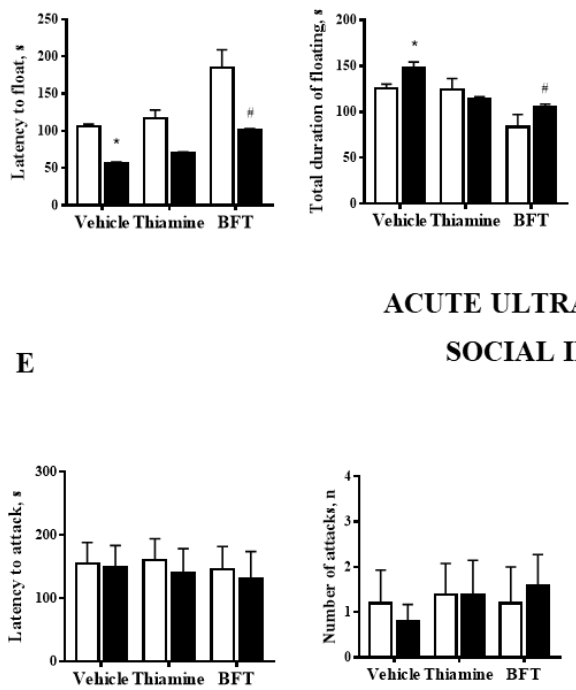

F

F FORCED SWIM

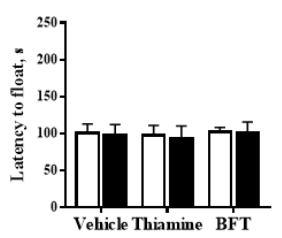

ACUTE ULTRASOUND EXPOSURE

SOCIAL INTERACTION
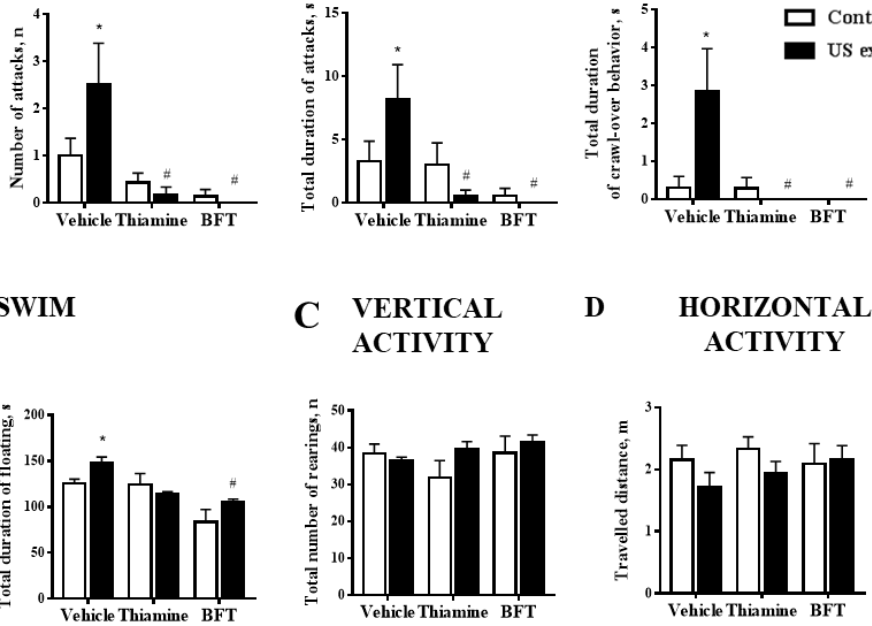

C VERTICAL

D

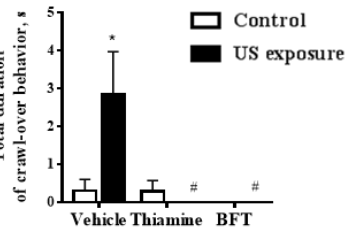

D HORIZONTAL ACTIVITY
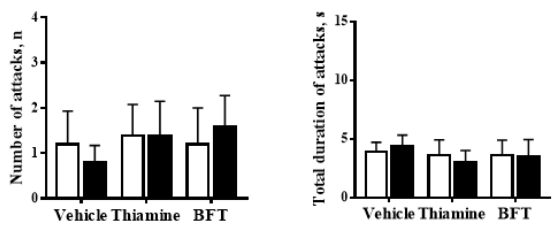

G VERTICAL ACTIVITY
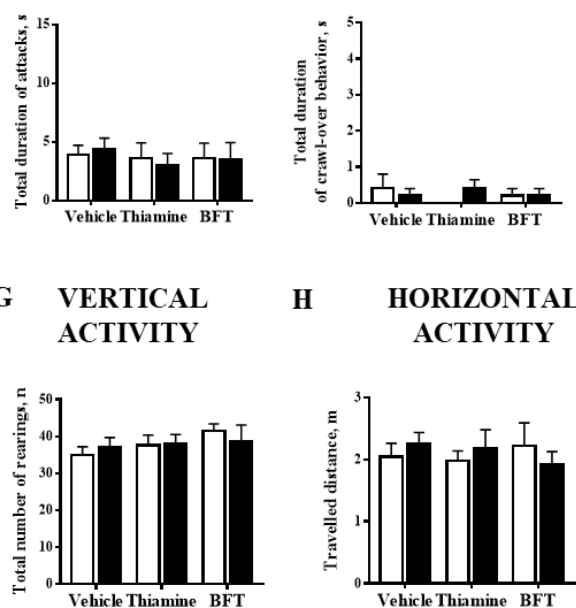

H HORIZONTAL ACTIVITY

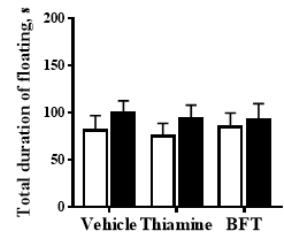

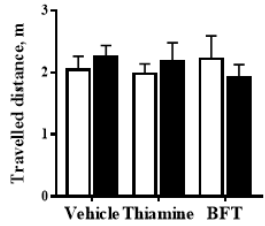


Figure 2. Ultrasound exposure induces aggressive and depressive-like behavioral changes in mice. (A) In the social interaction test, stressed vehicle-treated mice showed a significant decrease of the latency to attack, number of attacks, total duration and averaged duration of attacks in comparison to controls $\left({ }^{*} \mathrm{p}<0.05\right.$ vs. control, two-way ANOVA and post hoc Tukey's test). Stressed thiamineand benfotiamine-treated animals exhibited no change from control mice in these parameters and displayed significant differences from the stressed vehicle-treated group ( $\# \mathrm{p}<0.05$ vs. stressed group, two-way ANOVA and post hoc Tukey's test). (B) In the forced swim test, in comparison to control group, stressed vehicle-treated mice showed a significant decrease of the latency to float and total duration of floating $\left({ }^{*} \mathrm{p}<0.05\right.$ vs. control, two-way ANOVA and post hoc Tukey's test). Stressed thiamine- and benfotiamine-treated groups displayed no changes from control mice in these parameters and displayed significant differences from stressed vehicle-treated group $(\# p<0.05$ vs. stressed group, two-way ANOVA and post hoc Tukey's test). (C) There were no significant changes between groups in total number of rears ( $p>0.05$ vs. stressed group, two-way ANOVA and post hoc Tukey's test) (D) There were no significant changes between groups in travelled distance ( $p>0.05$ vs. stressed group, two-way ANOVA and post hoc Tukey's test; 7-8 animals per group were used) (E-H) A $24 \mathrm{~h}$ acute ultrasound exposure did not lead to any changes in parameters of aggressive behavior in the social interaction test, floating in the forced swim test, vertical or horizontal activity in the novel cage test ( $p>0.05$ vs. stressed group, two-way ANOVA and post hoc Tukey's test; 7 animals per group were used). Bars are Mean \pm SEM.

In the social interaction test, ultrasound-exposed vehicletreated mice showed a significant decrease in the number and total duration of social contacts in comparison to controls $\left({ }^{*} p<0.05\right.$ vs. control, two-way ANOVA and post hoc Tukey's test; see Supplementary material; Supplemental Fig.7). Ultrasoundexposed benfotiamine-treated, but not thiamine-treated animals exhibited no change from control mice in these parameters and displayed significant differences from the ultrasound-exposed 
vehicle-treated group ( $\# \mathrm{p}<0.05$ vs. ultrasound-exposed group, two-way ANOVA and post hoc Tukey's test). There were no significant changes between groups in latency to interact ( $p>0.05$ vs. ultrasound-exposed group, two-way ANOVA and post hoc Tukey's test).

In the forced swim test, a two-way ANOVA revealed a significant interaction between ultrasound and treatment, in the latency to float $\left(\mathrm{F}_{2,37}=3.427, \mathrm{p}=0.0428\right)$ and duration of floating behaviour $\left(\mathrm{F}_{2,37}=3.695, \mathrm{p}=0.0342\right.$, two-way ANOVA $)$. ANOVA revealed a significant ultrasound effect on the latency to float $\left(\mathrm{F}_{1,37}=36.03, \mathrm{p}<0.0001\right)$ and the duration of floating $\left(\mathrm{F}_{1,37}=4.405\right.$, $\mathrm{p}=0.0425$, two-way ANOVA; Fig. 2B). Chronic ultrasound exposure resulted in significant prolongation of the duration of floating and shortened latency to float, indicating increased helplessness in the ultrasound-exposed group. ANOVA revealed significant treatment effect for latency to float $\left(\mathrm{F}_{2,37}=17\right.$, $\mathrm{p}<0.0001)$ and total duration of floating $\left(\mathrm{F}_{2,37}=16.9, \mathrm{p}<0.0001\right.$, two-way ANOVA; Fig. 2B). When the ultrasound-exposed groups were treated with thiamine or benfotiamine, the exhibited decreased duration of floating and an increased latency to float, which is suggestive of an anti-depressant-like effect on the ultrasound-induced behavioral changes.

Post-hoc analysis revealed significant decrease of latency to float in ultrasound-exposed non-treated group in comparison with naïve mice ( $\mathrm{p}=0.241$, Tukey's test) and a significant increase of this parameter in ultrasound-exposed group treated with 
benfotiamine ( $\mathrm{p}=0.0489$, Tukey's test; Fig.2B), but not thiamine $(\mathrm{p}=0.3019$, Tukey's test) in comparison with ultrasound-exposed non-treated group. Post hoc analysis also revealed significant increase of total duration of floating in ultrasound-exposed nontreated group in comparison with naïve mice ( $\mathrm{p}=0.444$, Tukey's test) and a significant decrease of this parameter in ultrasoundexposed group treated with benfotiamine $(\mathrm{p}=0.0019$, Tukey's test; Fig.2B), but not thiamine ( $\mathrm{p}=0.0513$, Tukey's test) in comparison with ultrasound-exposed non-treated group.

A comparison of exploratory rearing activity by ANOVA revealed no interaction between ultrasound and treatment $\left(\mathrm{F}_{2,37}=1.116, \quad \mathrm{p}=0.3383\right)$, no significant ultrasound effect $\left(\mathrm{F}_{1,37}=1.309, \mathrm{p}=0.2599\right)$ and no significant treatment effect $\left(\mathrm{F}_{2,37}=0.8919, \mathrm{p}=0.4185\right.$, two-way ANOVA; Fig.2C), indicating a lack of non-specific changes in vertical locomotor activity. For horizontal activity, there also was no interaction between ultrasound and treatment $\left(\mathrm{F}_{2,37}=0.6669, \mathrm{p}=0.5193\right)$, as well as no significant ultrasound effect $\left(\mathrm{F}_{1,37}=1.612, \mathrm{p}=0.2122\right)$ and no significant treatment effect $\left(\mathrm{F}_{2,37}=0.4536, \mathrm{p}=0.6388\right.$, two-way ANOVA; Fig.2D). Furthermore, mice exposed to the ultrasound for just $24 \mathrm{~h}$ did not show any behavioural changes in any of the parameters measured, regardless of treatment (Fig.2E-G; see Supplementary material, Supplementary Table 3). 
3.2. Increased brain oxidative stress markers in mice with ultrasound-induced aggression and effects of thiamine compounds

Following the behavioural tests, the brains of these mice were studied for protein carbonyl and total glutathione content, and markers of oxidative stress in five brain structures. There was a significant interaction between ultrasound and treatment in protein carbonyl content in prefrontal cortex $\left(\mathrm{F}_{2,31}=5.154\right.$, $\mathrm{p}=0.0117)$, dorsal raphe $\left(\mathrm{F}_{2,31}=8.279, \mathrm{p}<0.0013\right)$ and striatum $\left(\mathrm{F}_{2,31}=4.824, \mathrm{p}<0.0193\right)$, but no significant interaction between ultrasound and treatment in the hippocampus $\left(\mathrm{F}_{2,31}=1.129\right.$, $\mathrm{p}=0.3362)$ and amygdala $\left(\mathrm{F}_{2,31}=1.254, \mathrm{p}=0.2993\right.$, two-way ANOVA). There was a significant ultrasound effect in prefrontal cortex $\left(\mathrm{F}_{1,31}=4.162, \mathrm{p}=0.049\right.$; Fig.3A), dorsal raphe $\left(\mathrm{F}_{1,31}=43.05\right.$, $\mathrm{p}<0.0001$; Fig.3D) and striatum $\left(\mathrm{F}_{1,31}=24.32, \mathrm{p}<0.0\right.$; Fig.3E), where protein carbonyl content was significantly elevated. Furthermore, ANOVA revealed significant treatment effect in prefrontal cortex $\left(\mathrm{F}_{2,31}=3.356, \mathrm{p}=0.0479\right.$; Fig.3A), amygdala $\left(\mathrm{F}_{2,31}=12.16, \mathrm{p}=0.0001\right.$; Fig.3C), dorsal raphe $\left(\mathrm{F}_{2,31}=13.58\right.$, $\mathrm{p}<0.0001$; Fig.3D) and striatum $\left(\mathrm{F}_{2,31}=12.61, \mathrm{p}<0.0001\right.$; Fig.3E). These data show that ultrasound exposure increases oxidative stress in limbic brain structures, which could be ameliorated by treatment with thiamine or benfotiamine. Notably, only ultrasound-exposed mice treated with benfotiamine displayed a significant decrease of protein carbonyl content in amygdala $(\mathrm{p}=0.038$, post hoc Tukey's test) and dorsal raphe $(\mathrm{p}<0.0001$, post hoc Tukey's test), in comparison with the vehicle-treated control 
group, suggesting superior effects of benfotiamine over that of thiamine.

A

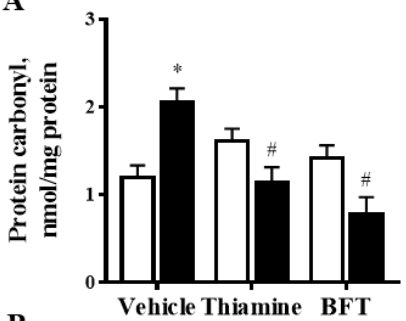

B

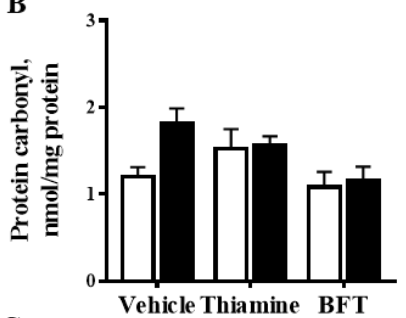

C

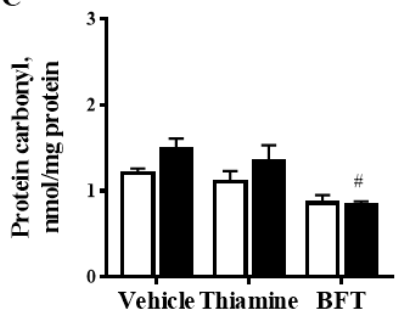

D

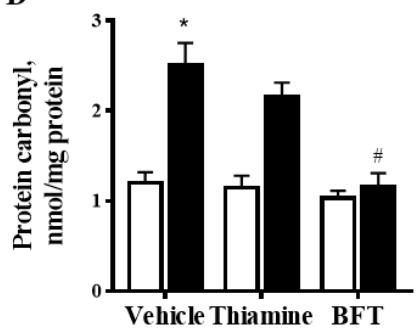

$\mathbf{E}$

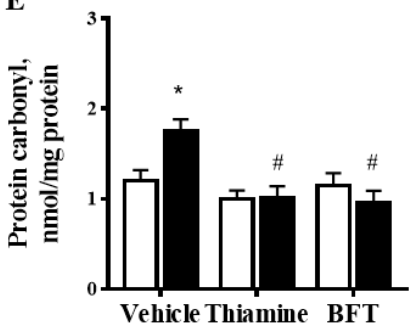

PREFRONTAL F

CORTEX

HIPPOCAMPUS

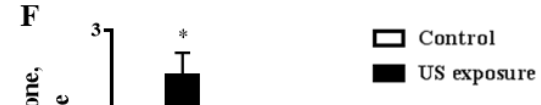

G

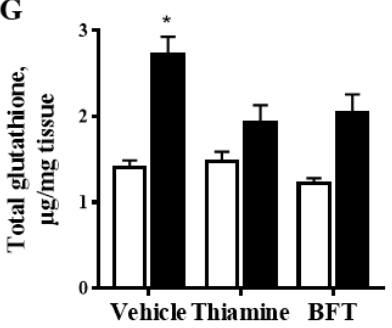

AMYGDALA H

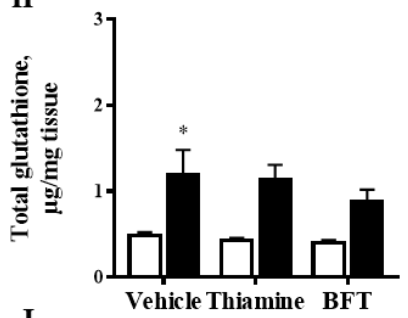

DORSAL RAPHE I

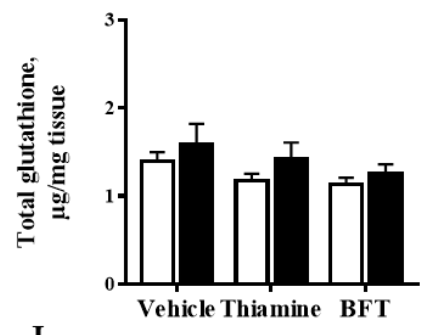

STRIATUM

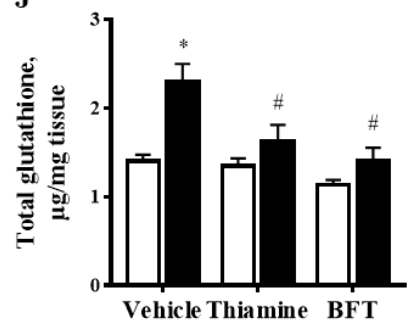


Figure 3. Increased brain oxidative stress markers in mice subjected to the ultrasound exposure and effects of thiamine compounds. (A) Stressed vehicle-treated mice demonstrated increased protein carbonyl contents in the prefrontal cortex in comparison with controls (*p< $<0.05$ vs. control, two-way ANOVA and post hoc Tukey's test). Stressed thiamine- and benfotiamine-treated mice displayed no changes from control mice in protein carbonyl contents in prefrontal cortex and displayed significantly lower values for this parameter as compared with the stressed vehicle-treated group $(\# p<0.05$ vs. stressed group, two-way ANOVA and post hoc Tukey's test). (B) There were no significant changes between groups in protein carbonyl contents in hippocampus ( $p>0.05$ vs. stressed group, two-way ANOVA and post hoc Tukey's test). (C) Benfotiamine-treated, but not thiamine-treated, stressed mice displayed significant differences from stressed vehicle-treated group in protein carbonyl contents in amygdala $(\# p<0.05$ vs. stressed group, two-way ANOVA and post hoc Tukey's test). (D) Stressed vehicle-treated mice demonstrated increased protein carbonyl contents in the dorsal raphe in comparison with controls $\left({ }^{*} p<0.05\right.$ vs. control, two-way ANOVA and post hoc Tukey's test). Benfotiamine-treated, but not thiamine-treated, stressed mice displayed significant differences from the stressed vehicle-treated group in protein carbonyl contents in dorsal raphe $(\# p<0.05$ vs. stressed group, two-way ANOVA and post hoc Tukey's test). (E) Stressed vehicle-treated mice demonstrated increased protein carbonyl contents in the striatum in comparison with controls $\left({ }^{*} \mathrm{p}<0.05\right.$ vs. control, twoway ANOVA and post hoc Tukey's test). Stressed thiamine- and benfotiamine-treated mice exhibited no changes from control mice in protein carbonyl contents in striatum and displayed significantly lower values of this parameter as compared with stressed vehicle-treated group $(\# p<0.05$ vs. stressed group, two-way ANOVA and post hoc Tukey's test; 6-7 animals per group were used). (F) Stressed vehicletreated mice exhibited significantly increased total glutathione contents in prefrontal cortex in comparison to the controls $\left({ }^{*} \mathrm{p}<0.05\right.$ vs. control, two-way ANOVA and post hoc Tukey's test). Benfotiaminetreated stressed mice exhibited no difference in total glutathione of the prefrontal cortex and showed significant decrease in values as compared with the stressed vehicle-treated group ( $\# p<0.05$ vs. stressed group, two-way ANOVA and post hoc Tukey's test). (G) Stressed vehicle-treated mice exhibited significantly increased total glutathione contents in hippocampus in comparison to the controls $\left({ }^{*} p<0.05\right.$ vs. control, two-way ANOVA and post hoc Tukey's test). (H) Stressed, vehicle-treated mice exhibited significantly increased total glutathione 
contents in amygdala in comparison to the controls (* $\mathrm{p}<0.05 \mathrm{vs.} \mathrm{control,}$ two-way ANOVA and post hoc Tukey's test). (I) There were no significant differences between groups in total glutathione contents in the dorsal raphe ( $>0.05$ vs. stressed group, two-way ANOVA and post hoc Tukey's test). (J) Stressed, vehicle-treated mice exhibited significantly increased total glutathione contents in the striatum in comparison to the controls (*p<0.05 vs. control, two-way ANOVA and post hoc Tukey's test). Stressed thiamine- and benfotiamine-treated mice exhibited no significant differences from control mice in total glutathione contents in striatum and displayed significantly lower measures of total glutathione than stressed vehicle-treated group $(\# p<0.05$ vs. stressed group, two-way ANOVA and post hoc Tukey's test; 6-7 animals per group were used). Bars are Mean \pm SEM.

Post-hoc analysis revealed significant increase of protein carbonyl content in prefrontal cortex in ultrasound-exposed nontreated group in comparison with naïve mice ( $p=0.0413$, Tukey's test) and a significant decrease of this parameter in the ultrasound-exposed group treated with thiamine $(p=0.0161$, Tukey's test) or benfotiamine ( $p=0.0138$, Tukey's test; Fig.3A) in comparison with the ultrasound-exposed non-treated group. Notably, only ultrasound-exposed mice treated with benfotiamine displayed a significant decrease of protein carbonyl content in amygdala ( $p=0.038$, post hoc Tukey's test) and dorsal raphe $(p<0.0001$, post hoc Tukey's test), in comparison with the ultrasound-exposed non-treated group, but not in the thiaminetreated ultrasound-exposed mice ( $\mathrm{p}=0.9543$, post hoc Tukey's test; Fig.3C). Post-hoc analysis also revealed significant increase of protein carbonyl content in the dorsal raphe in the ultrasoundexposed non-treated group in comparison with naïve mice 
( $<<0.0001$, Tukey's test). Again, only ultrasound-exposed mice treated with benfotiamine ( $<<0.0001$, post hoc Tukey's test), but not thiamine ( $\mathrm{p}=0.6789$, post hoc Tukey's test; Fig.3D) displayed a significant decrease of protein carbonyl content in dorsal raphe, in comparison with the ultrasound-exposed non-treated group, suggesting superior effects of benfotiamine over that of thiamine. There also was a significant increase of protein carbonyl content in prefrontal cortex in ultrasound-exposed non-treated group in comparison with naïve mice ( $\mathrm{p}=0.0002$, Tukey's test) and a significant decrease of this parameter in ultrasound-exposed group treated with thiamine $(\mathrm{p}=0.0002$, Tukey's test $)$ or benfotiamine ( $<00.0001$, Tukey's test; Fig. 3E) in comparison with ultrasound-exposed non-treated group.

There was a significant interaction between stress and treatment in total glutathione content in prefrontal cortex $\left(\mathrm{F}_{2,33}=3.834, \mathrm{p}=0.834\right)$, and striatum $\left(\mathrm{F}_{2,33}=4.824, \mathrm{p}=0.0145\right)$, and no significant interaction between ultrasound and treatment in hippocampus $\quad\left(\mathrm{F}_{2,33}=2.661, \quad \mathrm{p}<0.0848\right)$ and in amygdala $\left(\mathrm{F}_{2,33}=0.4012, \mathrm{p}=0.6728\right)$ and dorsal raphe $\left(\mathrm{F}_{2,33}=0.1137\right.$, $\mathrm{p}=0.8929$, two-way ANOVA). ANOVA revealed a significant ultrasound effect within the prefrontal cortex $\left(\mathrm{F}_{1,33}=53.22\right.$, $\mathrm{p}<0.0001$; Fig.3F), hippocampus ( $\mathrm{F}_{1,33}=69.65$, $\mathrm{p}<0.0001$; Fig.3G), amygdala $\quad\left(\mathrm{F}_{1,33}=31.51, \quad \mathrm{p}<0.0001 ; \quad\right.$ Fig. $\left.3 \mathrm{H}\right)$ and striatum $\left(\mathrm{F}_{1,33}=24.32\right.$, $\mathrm{p}<0.0001$; Fig.3J), where protein carbonyl content was significantly elevated in ultrasound-exposed group. Furthermore, ANOVA revealed significant treatment effect in 
prefrontal cortex $\left(\mathrm{F}_{2,33}=16.26, \mathrm{p}<0.0001\right.$; Fig. $\left.3 \mathrm{~F}\right)$ and in striatum $\left(\mathrm{F}_{2,33}=12.61, \mathrm{p}<0.0001 ;\right.$ Fig.3J $)$. At the same time, ANOVA revealed that there was no significant treatment effect in amygdala ( $\mathrm{F}_{2,33}=1.114, \mathrm{p}=0.3402$; two-way ANOVA; Fig. $\left.3 \mathrm{H}\right)$, and, though close, hippocampus $\left(\mathrm{F}_{2,33}=2.878, \mathrm{p}=0.0704\right.$; Fig.3G) and dorsal raphe $\left(\mathrm{F}_{2,33}=2.783\right.$, $\mathrm{p}=0.0764$; Fig.3I).

Post hoc analysis revealed significant increase of total glutathione content in prefrontal cortex in the ultrasoundexposed non-treated group in comparison with naïve mice $(p<0.0001$, Tukey's test $)$ and a significant decrease of this parameter in the ultrasound-exposed group treated with benfotiamine $(\mathrm{p}=0.0001$, Tukey's test), but not thiamine $(p=0.0527$, Tukey's test; Fig.3F) in comparison with the ultrasound-exposed non-treated group. It also revealed significant increase of total glutathione content in hippocampus $(\mathrm{p}<0.0001$, Tukey's test; Fig. $3 \mathrm{G})$ and amygdala ( $=0.0066$, Tukey's test; Fig.3H) in ultrasound-exposed non-treated group in comparison with naïve mice. There also was a significant increase of total glutathione content in striatum in the ultrasound-exposed non-treated group in comparison with naïve mice $(p<0.0001$, Tukey's test) and a significant decrease of this parameter in the ultrasound-exposed group treated with thiamine $(\mathrm{p}=0.0109$, Tukey's test) or benfotiamine ( $\mathrm{p}=0.0004$, Tukey's test; Fig.3J) in comparison with ultrasound-exposed non-treated group.

Thus, in a similar manner to the results obtained in the protein carbonyl assays, these data showed an increase of 
oxidative stress measures after 3-weeks of ultrasound exposure, which could be prevented by treatment with thiamine or benfotiamine. Again, only benfotiamine-treated ultrasoundexposed mice displayed a significant decrease of total glutathione content in prefrontal cortex in comparison with the vehicletreated control group. These data suggest that benfotiamine seems to have greater efficacy in comparison to thiamine. Mice exposed to the acute 24-h ultrasound exposure and/or administration of thiamine compounds did not show any changes in the parameters measured (Supplemental Fig.8, Supplementary Table 4).

3.3. Altered brain expression of AMPA receptor subunits and 5-HT6 receptor during ultrasound-induced aggression and effects of thiamine compounds

ANOVA revealed significant interaction between ultrasound and treatment for the mRNA levels of GluA1 subunit in the prefrontal cortex $\left(\mathrm{F}_{2,33}=4.96, \mathrm{p}<0.0131\right)$ and hippocampus $\left(\mathrm{F}_{2,33}=5.337, \mathrm{p}<0.0098\right.$, two-way ANOVA). More specifically, the mRNA levels of GluA1 subunit in both prefrontal cortex $\left(\mathrm{F}_{1,33}=5.081, \mathrm{p}=0.0310\right.$; Fig. $\left.4 \mathrm{~A}\right)$ and hippocampus $\left(\mathrm{F}_{1,33}=4.662\right.$, $\mathrm{p}=0.382$, two-way ANOVA; Fig.4B) was decreased after ultrasound exposure. ANOVA also revealed significant effect of treatment on the mRNA levels of GluA1 subunit in the prefrontal cortex $\left(\mathrm{F}_{2,33}=7.699, \quad \mathrm{p}=0.0018 ; \quad\right.$ Fig.4A $)$ and hippocampus $\left(\mathrm{F}_{2,33}=5.785, \mathrm{p}=0.007\right.$, two-way ANOVA; Fig.4B). 
PREFRONTAL CORTEX

A

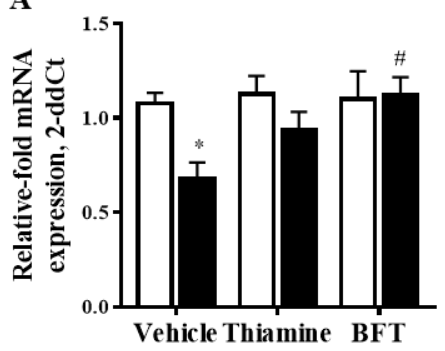

C

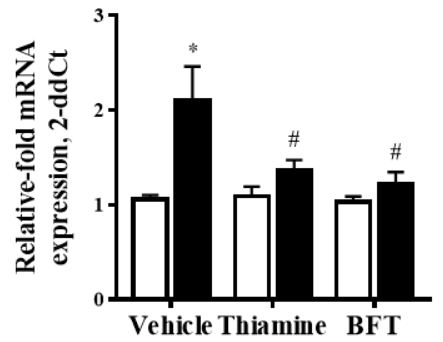

$\mathbf{E}$

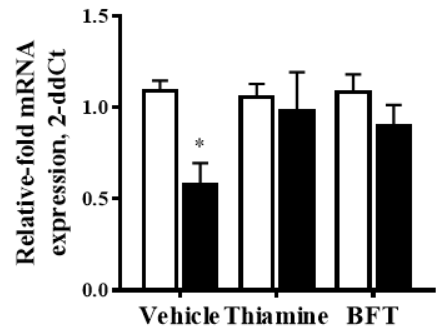

G

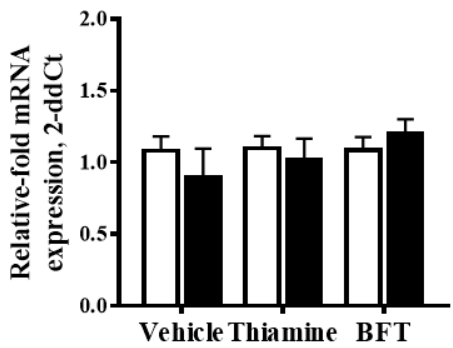

GluA1 B

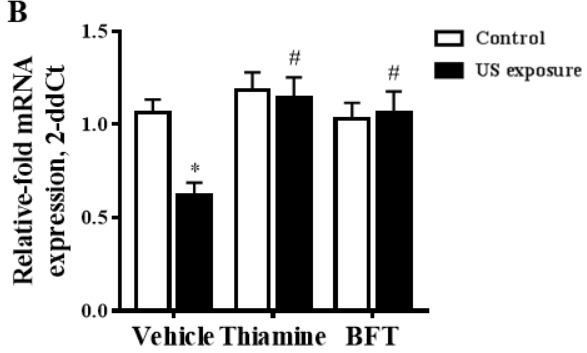

GluA2 D

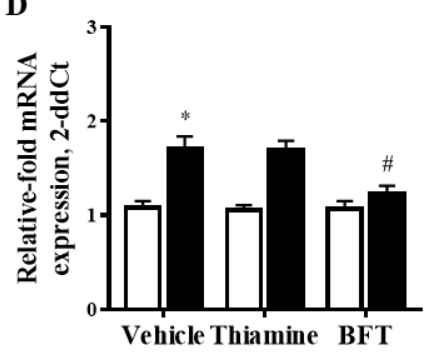

GluA3

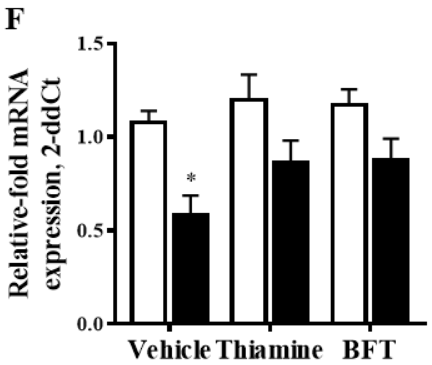

GluA4 H

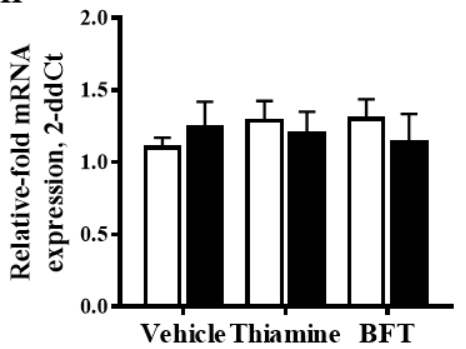

Figure 4. Altered brain expression of GluA1, GluA2, GluA3 and GluA4 subunits of AMPA receptor during stress-induced aggression and effects of thiamine compounds. (A) Stressed, vehicle-treated mice displayed a significant decrease in GluA1 mRNA concentrations in the prefrontal cortex $\left({ }^{*} \mathrm{p}<0.05\right.$ vs. control, two-way 
ANOVA and post hoc Tukey's test). In stressed benfotiamine-treated mice, GluA1 mRNA concentrations in the prefrontal cortex were unchanged as compared with the control and displayed significant differences from the stressed vehicle-treated group $(\# p<0.05$ vs. stressed group, two-way ANOVA and post hoc Tukey's test). (B) Stressed, vehicle-treated mice displayed a significant decrease in GluA1 mRNA concentrations in the hippocampus (*p<0.05 vs. control, two-way ANOVA and post hoc Tukey's test). In stressed thiamine- and benfotiamine-treated mice, GluA1 mRNA concentrations in the prefrontal cortex were unchanged as compared with the control and displayed significant differences from the stressed vehicle-treated group $(\# p<0.05$ vs. stressed group, two-way ANOVA and post hoc Tukey's test). (C) Stressed, vehicle-treated mice demonstrated a significant increase of GluA2 mRNA concentration in the prefrontal cortex (*p<0.05 vs. control, two-way ANOVA and post hoc Tukey's test). Stressed thiamine- and benfotiamine-treated mice levels of expression were unchanged compared to control mice in GluA2 mRNA concentrations in the prefrontal cortex and displayed significant differences from the stressed, vehicle-treated group $(\# p<0.05$ vs. stressed group, two-way ANOVA and post hoc Tukey's test). (D) Stressed, vehicle-treated mice demonstrated a significant increase of GluA2 mRNA concentrations in the hippocampus (*p<0.05 vs. control, two-way ANOVA and post hoc Tukey's test). Stressed benfotiaminetreated mice showed significant differences from stressed vehicletreated group in GluA2 mRNA concentrations in hippocampus $(\# \mathrm{p}<0.05$ vs. stressed group, two-way ANOVA and post hoc Tukey's test). (E) Stressed vehicle-treated mice demonstrated a significant decrease of GluA3 mRNA concentrations in the prefrontal cortex $\left({ }^{*} \mathrm{p}<0.05\right.$ vs. control, two-way ANOVA and post hoc Tukey's test). (F) Stressed vehicle-treated mice demonstrated a significant decrease of GluA3 mRNA concentrations in the hippocampus $(* p<0.05$ vs. control, two-way ANOVA and post hoc Tukey's test). (G) There were no significant changes between groups in GluA4 mRNA concentrations in the prefrontal cortex ( $>0.05$ vs. stressed group, two-way ANOVA and post hoc Tukey's test). (H) There were no significant changes between groups in GluA4 receptor mRNA concentrations in hippocampus ( $>0.05$ vs. stressed group, two-way ANOVA and post hoc Tukey's test; 6-7 animals per group were used). Bars are Mean \pm SEM. 
ANOVA revealed significant interaction between ultrasound and treatment in the mRNA levels of GluA2 subunit in the prefrontal cortex $\left(\mathrm{F}_{2,33}=4.816, \mathrm{p}=0.0146\right)$ and hippocampus $\left(\mathrm{F}_{2,33}=4.402, \mathrm{p}=0.0202\right.$, two-way ANOVA). Ultrasound exposure increased the mRNA levels of GluA2 subunit in both prefrontal cortex $\left(\mathrm{F}_{1,33}=15.17, \quad \mathrm{p}=0.0005 ;\right.$ Fig.4C $)$ and hippocampus $\left(\mathrm{F}_{1,33}=42.86, \mathrm{p}<0.0001\right.$, two-way ANOVA; Fig.4D). Again, there was a significant treatment effect in the mRNA levels of GluA2 subunit in the prefrontal cortex $\left(\mathrm{F}_{2,33}=4.796, \mathrm{p}=0.0148\right.$; Fig. $\left.4 \mathrm{C}\right)$ and hippocampus $\left(\mathrm{F}_{2,33}=4.438, \mathrm{p}=0.0196\right.$, two-way ANOVA; Fig.4D).

There was no significant interaction between ultrasound and treatment in the mRNA levels of GluA3 subunit in the prefrontal cortex $\left(\mathrm{F}_{2,33}=2.274, \mathrm{p}=0.1118\right)$ and hippocampus $\left(\mathrm{F}_{2,33}=0.6422, \mathrm{p}=0.5326\right.$, two-way ANOVA). Although, ANOVA revealed a significant ultrasound effect on the mRNA levels of GluA3 subunit in prefrontal cortex $\left(\mathrm{F}_{1,33}=8.58, \mathrm{p}=0.0061\right.$; Fig.4E) and also hippocampus $\left(\mathrm{F}_{1,33}=19.86, \mathrm{p}<0.0001\right.$, two-way ANOVA; Fig.4F), where GluA3 expression was decreased after ultrasound exposure. At the same time there was no significant treatment effect in the mRNA levels of GluA3 subunit in the prefrontal cortex $\left(\mathrm{F}_{2,33}=1.778, \mathrm{p}=0.1848\right.$; Fig. $\left.4 \mathrm{E}\right)$.

ANOVA revealed no significant interaction between ultrasound and treatment in the mRNA levels of GluA4 subunit in the prefrontal cortex $\left(\mathrm{F}_{2,33}=0.7632, \mathrm{p}=0.4742\right)$ and hippocampus $\left(\mathrm{F}_{2,33}=0.6422, \mathrm{p}=0.5326\right.$, two-way ANOVA) where 
expression is known to be very low. Furthermore, ANOVA revealed no significant ultrasound effect on the mRNA levels of GluA4 subunit in both prefrontal cortex $\left(\mathrm{F}_{1,33}=0.2391, \mathrm{p}=0.6281\right.$; Fig.4G) and hippocampus $\left(\mathrm{F}_{1,33}=0.09418, \mathrm{p}=0.7609\right.$, two-way ANOVA; Fig.4H). There was no significant treatment effect in the mRNA levels of GluA4 subunit in the prefrontal cortex $\left(\mathrm{F}_{2,33}=0.8071, \mathrm{p}=0.4548\right.$; Fig. $\left.4 \mathrm{G}\right)$ and hippocampus $\left(\mathrm{F}_{2,33}=0.1456\right.$, $\mathrm{p}=0.865$, two-way ANOVA; Fig. $4 \mathrm{H})$.

Post hoc analysis revealed significant decrease of GluA1 expression level in prefrontal cortex in ultrasound-exposed nontreated group in comparison with naïve mice $(p=0.0077$, Tukey's test) and a significant increase of this parameter in the ultrasound-exposed group treated with thiamine $(p=0.0269$, Tukey's test) or benfotiamine ( $\mathrm{p}=0.0069$, Tukey's test; Fig.4A) in comparison with ultrasound-exposed non-treated group. There also was a significant decrease of GluA1 expression level in hippocampus in ultrasound-exposed non-treated group in comparison with naïve mice $(\mathrm{p}=0.0187$, Tukey's test) and a significant increase of this parameter in ultrasound-exposed group treated with benfotiamine ( $\mathrm{p}=0.0224$, Tukey's test), but not thiamine ( $p=0.1301$, Tukey's test; Fig.4B) in comparison with ultrasound-exposed non-treated group. There also was a significant increase of GluA2 expression level in hippocampus in ultrasound-exposed non-treated group in comparison with naïve mice ( $p=0.0025$, Tukey's test) and a significant decrease of this parameter in ultrasound-exposed group treated with thiamine 
$(\mathrm{p}=0.0446$, Tukey's test) or benfotiamine ( $\mathrm{p}=0.0110$, Tukey's test; Fig.4C) in comparison with ultrasound-exposed non-treated group. Post-hoc analysis revealed a significant increase of GluA2 expression level in hippocampus in ultrasound-exposed nontreated group in comparison with naïve mice ( $\mathrm{p}=0.0019$, Tukey's test) and a significant decrease of this parameter in ultrasoundexposed group treated with benfotiamine $(\mathrm{p}=0.0165$, Tukey's test), but not thiamine ( $p>0.999$, Tukey's test; Fig.4D) in comparison with ultrasound-exposed non-treated group. Finally, post-hoc analysis revealed a significant decrease of GluA3 expression level in prefrontal cortex $(\mathrm{p}=0.0155$, Tukey's test; Fig.4E) and hippocampus (p=0.0131, Tukey's test; Fig.4F) in ultrasound-exposed non-treated group in comparison with naïve mice.

While there was an interaction between ultrasound and treatment at the mRNA level, this was not true for GluA2 protein expression in the prefrontal cortex $\left(\mathrm{F}_{2,33}=2.394, \mathrm{p}=0.1128\right)$ or hippocampus $\quad\left(\mathrm{F}_{2,33}=0.1222, \mathrm{p}=0.8855\right.$, two-way ANOVA $)$. However, ANOVA did reveal a significant main effect of ultrasound effect in the GluA2 protein expression in the prefrontal cortex $\left(\mathrm{F}_{1,33}=8.929, \mathrm{p}=0.0064\right)$, but not in the hippocampus $\left(\mathrm{F}_{1,33}=0.1504, \mathrm{p}=0.7016\right.$, two-way ANOVA $)$. There was no significant treatment effect in the GluA2 protein expression in the prefrontal cortex $\left(\mathrm{F}_{2,33}=1.57, \mathrm{p}=0.2286\right)$ and hippocampus $\quad\left(\mathrm{F}_{2,33}=1.814, \quad \mathrm{p}=0.1846, \quad\right.$ two-way ANOVA; Supplemental Fig.9A). Post-hoc analysis revealed a significant 
increase of GluA2 protein expression in prefrontal cortex in ultrasound non-treated group in comparison with naïve mice $(p=0.004$, Tukey's test). However, immunohistochemical analysis of the distribution of the subunits that exhibited expression changes after ultrasound exposure (GluA1, GluA2, and GluA3) revealed that the subunits were internalized in in ultrasoundexposed animals compared the controls for GluA1 and GluA2, but not for GluA3. ultrasound-exposed, vehicle-treated mice also exhibited a significant decrease of GluA2 and GluA3, but not GluA1, on the cell surface, in CA1 zone of the hippocampus (Supplemental Figs. 12,13). Thus the ultrasound had an impact on the distribution of all of the subunits except GluA4.

There was no significant interaction between ultrasound and treatment for 5-HT6R mRNA levels in the prefrontal cortex $\left(\mathrm{F}_{2,33}=0.05771, \mathrm{p}=0.0944\right)$ and hippocampus $\left(\mathrm{F}_{2,33}=1.292\right.$, $\mathrm{p}=0.2883$ ). There was also no significant interaction between ultrasound and treatment in 5-HT6 receptor relative fold protein concentrations in the prefrontal cortex $\left(\mathrm{F}_{2,30}=1.756, \mathrm{p}=0.19\right)$. Furthermore, ANOVA revealed no significant ultrasound effect in 5-HT6R mRNA concentrations in the prefrontal cortex $\left(\mathrm{F}_{1,33}=0.1272, \quad \mathrm{p}=0.7236\right)$ and hippocampus $\left(\mathrm{F}_{1,33}=0.4828\right.$, p=0.4920; Supplemental Fig.10A), but, at the same time, ANOVA did reveal that there was a significant ultrasound effect in 5-HT6 receptor relative fold protein concentrations in the prefrontal cortex $\left(\mathrm{F}_{1,30}=7.351, \mathrm{p}=0.011\right)$ and hippocampus $\left(\mathrm{F}_{1,30}=6.281\right.$, $\mathrm{p}=0.0179$, two-way ANOVA; Supplemental Fig.10B). Finally, 
ANOVA revealed no significant treatment effect in 5-HT6R mRNA concentrations in the prefrontal cortex $\left(\mathrm{F}_{2,33}=0.2007\right.$, $\mathrm{p}=0.8191)$ and hippocampus $\left(\mathrm{F}_{2,33}=0.6105, \mathrm{p}=0.5491\right.$; Fig.10B of Supplementary file), as well as in 5-HT6 receptor relative fold protein concentrations in the prefrontal cortex $\left(\mathrm{F}_{2,30}=1.247\right.$, $\mathrm{p}=0.302)$ and hippocampus $\left(\mathrm{F}_{2,30}=0.9648, \mathrm{p}=0.3926\right.$, two-way ANOVA; Supplemental Fig.10B). Post-hoc analysis revealed significant decrease of 5-HT6 protein expression in prefrontal cortex ( $\mathrm{p}=0.0124$, Tukey's test) and hippocampus ( $\mathrm{p}=0.0427$, Tukey's test) in ultrasound-exposed non-treated group in comparison with naïve mice. Thus, a 3 -week long ultrasound exposure resulted in altered expression of the different subunits of the AMPA receptor and some changes in the expression of $5 \mathrm{HT}$ 6 receptor in the limbic structures of the brain. Treatment with thiamine or benfotiamine prevented some of these ultrasoundinduced changes, but the lack of effect of thiamine or benfotiamine on 5HT-6 receptor expression suggests that it is not contributing to the aggressive behaviour in these animals. Mice exposed to the acute $24 \mathrm{~h}$ ultrasound exposure and/or administration of thiamine compounds did not show any changes in any of the parameters measured (see Supplementary material, Supplementary Table 5). 
3.4. Suppressed expression of plasticity factors resulting from the ultrasound exposure and effects of thiamine drugs

ANOVA revealed a significant interaction between ultrasound and treatment in the protein concentration of PSD95 in hippocampus $\left(\mathrm{F}_{2,30}=5.024, \mathrm{p}=0.0131\right)$, but not in the prefrontal cortex $\left(\mathrm{F}_{2,30}=1.816, \mathrm{p}=0.1802\right.$, two-way ANOVA). Furthermore, ANOVA revealed a significant ultrasound effect in both prefrontal cortex $\left(\mathrm{F}_{1,30}=9.017, \quad \mathrm{p}=0.0054 ; \quad\right.$ Fig.5A $)$ and hippocampus $\left(\mathrm{F}_{1,30}=5.252\right.$, p=0.0291, two-way ANOVA; Fig.5B) as ultrasound-exposed mice displayed a significantly decreased protein concentration of PSD95. There also was a significant treatment effect in the hippocampus $\left(\mathrm{F}_{2,30}=3.326, \mathrm{p}=0.0496\right.$, Fig.5B) but, though close, not in the prefrontal cortex $\left(\mathrm{F}_{2,30}=3.177\right.$, $\mathrm{p}=0.0561$, Fig.5A), indicating the efficacy of treatment with thiamine and benfotiamine against ultrasound-induced changes.

ANOVA revealed a significant interaction between ultrasound and treatment in protein concentration of PSA-NCAM in hippocampus $\left(\mathrm{F}_{2,30}=4.335, \mathrm{p}=0.0222\right)$. Furthermore, ANOVA revealed a significant ultrasound effect in both prefrontal cortex $\left(\mathrm{F}_{1,30}=4.49, \mathrm{p}=0.0425\right.$; Fig.5C) and hippocampus $\left(\mathrm{F}_{1,30}=7.071\right.$, $\mathrm{p}=0.0124$, two-way ANOVA; Fig.5D) where ultrasound-exposed mice exhibited significantly decreased protein concentration of PSA-NCAM. There also was a significant treatment effect in both prefrontal cortex $\left(\mathrm{F}_{2,30}=5.324, \quad \mathrm{p}=0.0105, \quad\right.$ Fig.5C $) \quad$ and hippocampus $\quad\left(\mathrm{F}_{2,30}=3.633, \mathrm{p}=0.0386\right.$, Fig.5D), once again indicating the effectiveness of treatment with thiamine and 
benfotiamine against ultrasound-induced changes. Remarkably, only benfotiamine-treated ultrasound-exposed mice demonstrated a significant increase of hippocampal concentrations of PSA-NCAM in comparison with the ultrasoundexposed vehicle-treated group in hippocampus $(\mathrm{p}=0.0166$, post hoc Tukey's test).

PREFRONTAL CORTEX

A

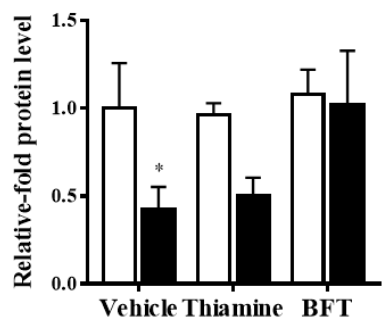

C

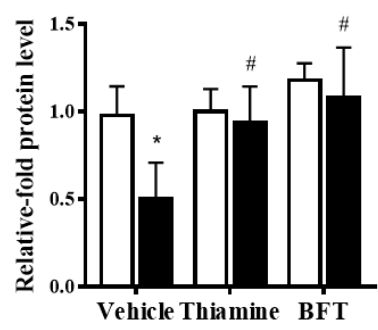

$\mathbf{E}$

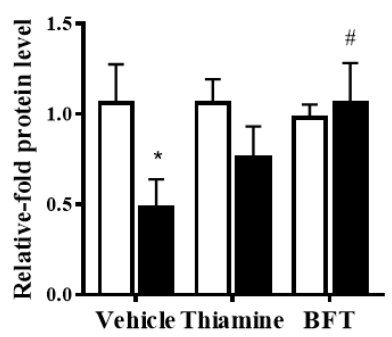

HIPPOCAMPUS

PSD95 B

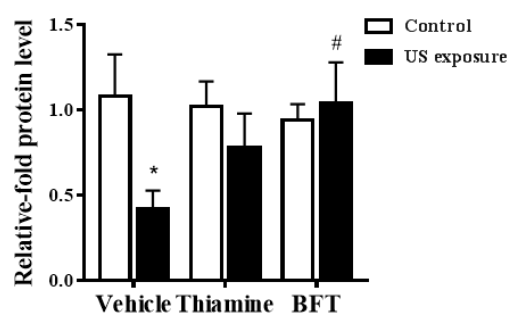

PSA-NCAM D

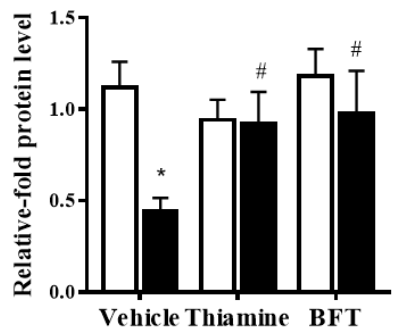

b-CATENIN F

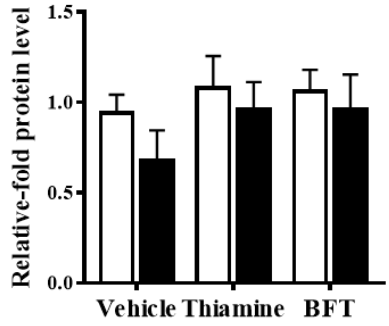


Figure 5. Suppressed expression of plasticity factors resulting from the ultrasound and effects of thiamine drugs. (A) Stressed vehicle-treated mice displayed a significant decrease of PSD95 relative fold protein levels in the prefrontal cortex ( ${ }^{*} \mathrm{p}<0.05$ vs. control, two-way ANOVA and post hoc Tukey's test). (B) Stressed vehicletreated mice exhibited a significant decrease of PSD95 relative fold protein levels in the hippocampus $\left({ }^{*} \mathrm{p}<0.05 \mathrm{vs}\right.$. control, two-way ANOVA and post hoc Tukey's test). Benfotiamine-treated mice displayed no changes from control mice in PSD95 relative fold protein levels in the hippocampus and displayed significant differences from stressed vehicle-treated group (\#p<0.05 vs. stressed group, two-way ANOVA and post hoc Tukey's test). (C) Stressed vehicle-treated mice displayed a significant decrease of PSA-NCAM relative fold protein levels in the prefrontal cortex $\left({ }^{*} \mathrm{p}<0.05\right.$ vs. control, two-way ANOVA post hoc and Tukey's test). Thiamine- and benfotiamine-treated mice exhibited no change from control mice in PSA-NCAM relative fold protein levels in the prefrontal cortex and displayed significant differences from stressed vehicle-treated group (\#p<0.05 vs. stressed group, two-way ANOVA and post hoc Tukey's test). (D) Stressed vehicle-treated mice displayed a significant decrease of PSA-NCAM relative fold protein levels in the hippocampus $\left({ }^{*} p<0.05\right.$ vs. control, two-way ANOVA and post hoc Tukey's test). Benfotiamine-treated mice exhibited significant differences in PSA-NCAM relative fold protein levels in hippocampus from stressed vehicle-treated group $(\# p<0.05$ vs. stressed group, twoway ANOVA and post hoc Tukey's test). (E) Stressed vehicle-treated mice had a significant decrease of the B-catenin relative fold protein levels in prefrontal cortex (*p<0.05 vs. control, two-way ANOVA and post hoc Tukey's test). Benfotiamine-treated mice had no changes from control mice in the B-catenin relative fold protein levels in the prefrontal cortex and displayed significant differences from the stressed vehicletreated group $(\# p<0.05$ vs. stressed group, two-way ANOVA and post hoc Tukey's test). (F) There were no significant changes between groups in the B-catenin relative fold protein levels in the hippocampus ( $p>0.05$ vs. stressed group, two-way ANOVA and post hoc Tukey's test; 6 animals per group were used). Bars are Mean \pm SEM.

ANOVA revealed a significant interaction between ultrasound and treatment in protein concentration of B-catenin $\left(\mathrm{F}_{2,30}=5.297, \quad \mathrm{p}=0.0107\right), \quad \mathrm{a}$ significant ultrasound effect 
$\left(\mathrm{F}_{2,30}=4.424, \mathrm{p}=0.0439\right)$ and also a significant treatment effect $\left(\mathrm{F}_{2,30}=3.753, \mathrm{p}=0.0351\right.$, two-way ANOVA, Fig.5E) in prefrontal cortex. At the same time there was no interaction between ultrasound and treatment in protein concentration of B-catenin $\left(\mathrm{F}_{2,30}=0.7935, \mathrm{p}=0.4615\right)$ or, though close, a ultrasound effect $\left(\mathrm{F}_{2,30}=3.542, \mathrm{p}=0.0696\right)$ or treatment effect $\left(\mathrm{F}_{2,30}=2.577, \mathrm{p}=0.0928\right.$; two-way ANOVA; Fig.5F) in the hippocampus. Post-hoc analysis revealed significant decrease of PSD95 protein concentration in prefrontal cortex in ultrasound-exposed non-treated group in comparison with naïve mice ( $\mathrm{p}=0.0471$, Tukey's test; Fig.5A). There was a significant decrease of PSD95 protein concentration in hippocampus in ultrasound-exposed non-treated group in comparison with naïve mice ( $\mathrm{p}=0.0121$, Tukey's test) and a significant increase of this parameter in ultrasound-exposed group treated with benfotiamine ( $\mathrm{p}=0.0041$, Tukey's test), but not thiamine ( $\mathrm{p}=0.2333$, Tukey's test; Fig.5B) in comparison with ultrasound-exposed non-treated group.

There was a significant decrease of PSA-NCAM protein concentration in prefrontal cortex in ultrasound-exposed nontreated group in comparison with naïve mice ( $\mathrm{p}=0.0363$, Tukey's test) and a significant increase of this parameter in ultrasoundexposed group treated with thiamine ( $\mathrm{p}=0.0438$, Tukey's test) or benfotiamine ( $p=0.0111$, Tukey's test; Fig.5C) in comparison with ultrasound-exposed non-treated group. There also was a significant decrease of PSA-NCAM protein concentration in hippocampus in ultrasound-exposed non-treated group in 
comparison with naïve mice ( $p=0.0060$, Tukey's test) and a significant increase of this parameter in ultrasound-exposed group treated with thiamine $(p=0.0464$, Tukey's test $)$ or benfotiamine ( $p=0.0166$, Tukey's test; Fig.5D) in comparison with ultrasound-exposed non-treated group. Finally, there was a significant decrease of B-catenin protein concentration in hippocampus in ultrasound-exposed non-treated group in comparison with naïve mice ( $p=0.0149$, Tukey's test) and a significant increase of this parameter in ultrasound-exposed group treated with benfotiamine ( $\mathrm{p}=0.0026$, Tukey's test), but not thiamine $(p=0.0512$, Tukey's test; Fig.5E) in comparison with ultrasound-exposed non-treated group.

Mice exposed to the acute $24 \mathrm{~h}$ ultrasound exposure and/or the administration of thiamine compounds did not show any changes in any of the parameters measured (see Supplementary material, Supplementary Table 6).

\section{Discussion}

We found that mice subjected to the chronic ultrasound exposure exhibit aggressive behavior and, at a molecular level, increased expression in the brain of the oxidative stress markers protein carbonyl and total glutathione. These effects were found in vehicle-treated ultrasound-exposed mice, but not in the thiamine-treated and benfotiamine-treated ultrasound-exposed groups. Ultrasound-exposed vehicle-treated mice displayed elevated gene expression of the GluA2 subunit, although cell 
surface protein expression is reduced, and decreased protein expression of 5-HT6 receptor and the plasticity factors PSD95, PSA-NCAM and B-catenin, in the prefrontal cortex and the hippocampus. Again, these ultrasound effects were not observed in the thiamine-treated and benfotiamine-treated animals. Control experiments showed that a 24-h acute ultrasound exposure and/or administration of either compound did not affect any of the behavioural and molecular parameters measured in the mice.

Thus, the present study has revealed generalized increases of oxidative stress markers in the brain to parallel elevated scores of aggressive behaviour in mouse model emotional stress and these changes were reversed by the administration of thiamine and benfotiamine. Treatment with benfotiamine had a greater effect on reversing brain oxidative stress than thiamine, supporting previous observations that there may be increased bioavailability for benfotiaine in comparison to thiamine (Pan et al., 2010; Vignisse et al., 2017). The presence of increased brain oxidation stress markers with aggression in mice is in keeping with previous findings obtained in rodent models of naturalistic adversity (Diehl et al., 2012; Patki et al., 2013, 2016). For instance, Sprague Dawley rats subjected to social defeat stress, exhibited elevated concentrations of oxidative stress markers that correlated with their scores of aggressive behavior (Patki et al., 2016). Wistar rats subjected to maternal separation stress displayed elevated antioxidant enzymes activities in the 
hippocampus and exhibited impaired hippocampus-dependent behaviours (Diehl et al., 2012). Physical exercise was shown to counteract stress-induced effects in a rat model of maternal separation (Daniels et al., 2012). Other types of stress, that potentiate aggressiveness in small rodents, including chronic mild stress, were shown to increase oxidative stress measures in the brain as in the periphery (Rammal et al., 2010; Costa-Nunes et al., 2014; Cline et al., 2015a; Malki et al., 2016).

Chronic administration of thiamine and benfotiamine prevented pro-oxidative effects of the ultrasound exposure. Previous studies that employed the same dosing regime, and were of similar duration, reported an absence of a change in the brain content of thiamine mono-phosphate and thiamine di-phosphate, where the latter is an essential cofactor of brain metabolism (Volvert et al., 2008; Pan et al., 2010; Vignisse et al., 2017). Surprisingly, both treatments resulted in similar changes in brain thiamine concentrations, while bioavailability of benfotiamine is known to significantly exceed that of thiamine (Vignisse et al., 2017). From this outcome, it can be assumed that the present ameliorative effects of these compounds are not primarily reliant of the regulation of brain energy metabolism, to and more likely to be dependent on their non-co-enzyme extracellular effects. Indeed, studies of recent years suggest that there are wide spectrum of functions for thiamine in the CNS that are dependent on non-enzymatic mechanisms (Bunik, 2013; Bettendorff et al., 2014; Mkrtchyan et al., 2015). These effects are 
likely to have mediated the anti-oxidative and neuroprotective action of thiamine and benfotiamine reported here.

Thiamine is essential for normal brain function and its deficiency causes metabolic impairment, oxidative damage, and reduced adult hippocampal neurogenesis. While we suspect that the reversal of the AMPA subunit expression profile by thiamine and benfotiamine is likely to be a downstream consequence of the treatment, the $\mathrm{Ca}^{2+}$ permeability of AMPA receptors is determined by the presence of the GluA2 subunit (Hume et al. 1991). GluA2 is a critical subunit in determining many of the biophysical properties of AMPARs, including $\mathrm{Ca}^{2+}$ permeability, and AMPARs lacking GluA2 are more permeable to $\mathrm{Ca}^{2+}$ and $\mathrm{Zn}^{2+}$. Thiamine deficiency leads to increased $\mathrm{Ca}^{2+}$ entry via GluA2, which is thought to be a consequence of reduced thiaminedependent pre-mRNA editing that silences GluA2 (ADAR2dependent RNA editing of GluA2 is involved in thiamine deficiency-induced alteration of calcium dynamics). Thus the ultrasound exposure-induced increase in GluA2 mRNA is likely to result in increased AMPA signaling and treatment with thiamine and benfotiamine may increase pre-mRNA editing to suppress GluA2 mediated calcium currents.

While GluA2 mRNA was increased, GluA1 and GluA3 the levels of transcript and cell surface protein expression were reduced in ultrasound exposed mice. Surprisingly, the cell surface expression of GluA2 protein was also decreased. This seeming disparity probably reflects increased turnover of GluA2 protein in 
neuronal populations, which may have resulted in an increased demand for transcript. However, the discrepancy may also reflect increased expression in GluA2 mRNA expression in non-neuronal cell populations. Homomeric GluA2 subunits are responsible for the low conductance of AMPAR in activated microglia. GluA2 levels of expression are increased in microglia by the injections of LPS into mice and we know that stress activates microglia. The GluA2 subunits of the AMPARs, also expressed in astrocytes, are crucial determinants in controlling the calcium permeability and function (Fan et al., 1999). However, while GluA3 is increased in reactive astrocytes GluA2, where they have been measured, is unchanged (Newcombe et al., 2008). Concerning the differences that we observed between the regulation of subunit expression reported here in our study, several studies have suggested that these receptor subunits may play distinct roles in the regulation of AMPA receptor trafficking and synaptic plasticity. Thus, the ability for their expression to be differentially regulated, depending on demand and environmental conditions, is unsurprising. The GluA1 subunit is required for NMDA receptordependent synaptic delivery of AMPA receptors. In contrast, GluA2/GluA3 receptors seem to play a complementary role in the constitutive delivery pathway. Furthermore, in studies using GluA1 or GluA2 KO mice, it is clear that GluA1 and GluA2 act differentially in LTP formation (Toyoda et al., 2009). GluA2 incorporation into AMPA receptors seems to be particularly important in synaptic scaling, and, thus, changes in the ratios of 
GluA1 and GluA2 suggest that synaptic plasticity is likely to be altered following ultrasound exposure. Whatever the significance of the changes in levels of expression, what is very clear from our study is that treatment normalised receptor subunit expression.

An important non-enzymatic mechanism that may be important here is the previously described action of thiamine and benfotiamine on GSK-3B activities (Pan et al., 2010; Pavlov et al., 2017; Markova et al., 2017). This kinase phosphorylates Nrf2, a regulator of antioxidant response, that suppresses expression of Nrf2-dependent genes, and, consequently, the protection of neurons from oxidative stress (Rada et al., 2012; Beurel et al., 2015). A decrease of GSK-3B activity may then results in a reduction of oxidative stress, which may underpin the effects of the thiamine compounds on protein carbonyl and total glutathione concentrations in brains of the ultrasound-exposed mice.

Pro-oxidative changes in rodent stress models were previously reported to result in impaired social behaviours, elevated aggressiveness, depressive-like changes and suppression of brain plasticity (Daniels et al., 2012; Diehl et al., 2012; Patki et al., 2013, 2016; Cline et al., 2015a,b). In line with these data, we found decreased expression of plasticity factors in the hippocampus and in the prefrontal cortex. A reduction of morphological and molecular markers of brain plasticity was previously shown to accompany excessive oxidative stress and aggression measures in clinical and pre-clinical studies (Marais 
et al., 2009; Boufleur et al., 2013; Okazawa et al., 2014; Salminen et al., 2014). Here, we found suppressed gene and protein expression in plasticity markers whose function is also associated with brain oxidative stress and aggressive behavior.

For instance, ultrasound-exposed vehicle-treated mice had lowered expression of B-catenin, a plasticity factor that triggers oxidative stress cascades via forkhead box O (FOXO), an element of GSK-3B pathway, which known as pivotal modulator of neuroinflammation and apoptosis (Arnold et al., 2001; Lento et al., 2014; Beurel et al., 2015). Ultrasound-exposed vehicle-treated mice exhibited also decreased brain expression of PSD95, a scaffolding protein regulating synaptic development and remodeling, whose expression was recently demonstrated to be altered in rodent models of aggression (Been et al., 2016). Diminished expression of PSD95, as well as another important plasticity factor, NCAM and its functionally important polysialylated form PSA-NCAM, have been found to be associated with higher levels of aggression and territoriality in mice (Angata et al., 2004; Calandreau et al., 2010; Winkler et al., 2017), which is in keeping with the results reported here. These data support recently published findings showing that the chronic exposure of $\mathrm{BALB} / \mathrm{c}$ mice to the ultrasound of alternating frequencies resulted in decreased densities of Ki67- and DCX-positive cells in the hippocampus, altered expression of BDNF together with its receptor TrkB, increased density of Iba-1, a marker of microglial activation, elevated levels of IL-1B and IL-6 in the hippocampus 
and serum, elevated activities of GSK-3B/Akt/FOXO3a cascade as well as other changes (Pavlov et al., 2019).

Compromised plasticity mechanisms, resulting from stress experiences are suggested to hamper cognitive flexibility and ability to control behavior, thereby leading to excessive aggression (Fanning et al., 2017). In addition to other actions, thiamine and benfotiamine have also been reported to improve cognition and memory. Pro-cognitive drugs developed for Alzheimer disease, such as dimebon and DF302, have also been shown to reduce aggression and this would be in keeping with the actions of thiamine and benfotiamine observed here (Vignisse et al., 2011; Markova et al., 2017; Strekalova et al., 2018). The results with the pro-cognitive interventions resulted in modulation of AMPA- and 5-HT6 receptor-mediated neurotransmission, which was also observed after treatment with the thiamine compounds.

The role of AMPA- and 5-HT6 receptor-mediated neurotransmission in excessive aggression has been highlighted in previous rodent studies with corticosterone administration (Sun et al., 2018), naturalistic stress paradigms (Partyka et al., 2016; Shimizu et al., 2016), genetic manipulations (Vekovischeva et al., 2004, 2007; Adamczyk et al., 2012), chronic stress (CostaNunes, 2014; Zhang et al., 2015), pharmacological experiments (Upton et al., 2008; Yun and Rhim, 2011) and in clinical postmortem and association studies (Tsai et al., 1999; Garcia-Alloza et al., 2014; Marcos et al., 2018; Azenha et al., 2009; Ramirez, 
2013; Zhang et al., 2018). For instance, treatment with antagonists of AMPA/kainate receptor and manipulations of the 5-HT6 receptors were found to attenuate the aggressive behavior of mice subjected to the isolation stress (Adamczyk et al., 2012; Araki et al., 2014; Partyka et al., 2016; Shimizu et al., 2016) and social stress (Vekovischeva et al., 2007). Of note, regulatory effects of GSK-3B on AMPA receptor have also been reported recently (Khghlhyatian et al., 2018) that leads us to speculate that this pathway, in particular, may be responsible for the normalizing action of thiamine and benfotiamine on gene and protein expression of brain AMPA receptor observed in the present study.

The AMPA receptor GluA2, as well as 5-HT6 receptor, are regarded as having a central role in brain plasticity (Song and Huganir, 2002; Garcia-Alloza et al., 2014; Henley and Wilkinson, 2013; Zhang et al., 2015, 2018; Pereira et al., 2015; Meneses, 2017). Thus it seems probable that the anti-aggressive effects of the thiamine compounds are mediated by ameliorating the ultrasound-induced inhibition of plasticity mechanisms in the brain. This view is supported by the positive effects that have been observed for thiamine and benfotiamie on hippocampal plasticity in a rat stress model (Markova et al., 2017), as well as on the induction of neuroprotective mechanisms after CNS trauma (Boyko et al., 2018; Mktkachryan et al., 2018), neurotoxicity (Benton and Donohoe, 1999), insulin resistance 
(Abdou and Hazell, 2015) and neurodegeneration (Pan et al., 2010, 2016; Tapias et al., 2018).

Finally, it is of note that benfotiamine exerted more pronounced normalizing changes on protein carbonyl, total glutathione and expression of 5-HT6 receptor in the brain of ultrasound-exposed mice than treatment with the same concentration of thiamine. These data are in line with the previously reported superior effects of benfotiamine on hippocampal neurogenesis during predation stress (Vignisse et al., 2017) and in a model of tau-pathology (Tapias et al., 2018) compared to thiamine. These differences may be due to the increased bioavailability of benfotiamine, but similar brain levels of thiamine and its metabolites have reported after treatment with benfotiamine (Vignisse et al., 2017). Thus the differences may reflect altered activity of the non-enzymatic effects of benfotiamine, the analog-specific mechanisms underlying these differences remain to be explored.

\section{Conclusions}

Together, current work suggests the potential for therapeutic application of thiamine and its precursors, in particular, benfotiamine, in prevention and treatment of neuropsychiatric conditions associated with increased brain oxidative stress, including symptoms of excessive aggression. Apart from the glutamatergic, serotonergic and brain plasticity mechanisms reported here, other mechanisms could also be 
responsible for the effects reported here, such as antiinflammatory activities and the biosynthesis of monoamines that were previously described for thiamine and benfotiamine (Sanchez-Ramirez et al., 2006; Shoeb and Ramana, 2012; Abdou and Hazell, 2015). Given the accumulated clinical evidence of therapeutic efficacy and tolerability of thiamine and benfotiamine (Bunik, 2013; Ghaleiha et al., 2016; Pan et al., 2016), their usefulness in the prevention and therapy for overt aggression and other associated symptoms would come with very little associated risk and full translation, if successful, might be expected to have significant benefit at low cost.

\section{Acknowledgements}

The authors' work reported here was supported by the European Union's Seventh Framework Programme under Grant No. 602805 (Aggressotype), the Horizon 2020 Research and Innovation Programme under Grant No. 728018 (Eat2beNICE), (E+2016-KA107-SM to DP and LB), and "5-100" Russian Research Excellence program (to TS and KPL). RT-PCR and oxidative stress assays from the reported study were funded by RFBR according to the research project № 18-34-00275. We thank Thomas Ricker for valuable technical support with behavioural data analysis. LB is Research Director of the F.R.S.-FNRS (Belgium).

\section{Declaration of interests: None}




\section{Reference List}

Abdou E., Hazell A.S., 2015. Thiamine deficiency: an update of pathophysiologic mechanisms and future therapeutic considerations. Neurochem Res. 40, 353-361. doi: 10.1007/s11064-014-1430-z.

Adamczyk A., Mejias R., Takamiya K., Yocum J., Krasnova I.N., Calderon J., Cadet J.L., Huganir R.L., Pletnikov M.V., Wang T., 2012. GluA3-deficiency in mice is associated with increased social and aggressive behavior and elevated dopamine in striatum. Behav Brain Res. 229, 265-272. doi: 10.1016/j.bbr.2012.01.007.

Angata K., Long J.M., Bukalo O., Lee W., Dityatev A., Wynshaw-Boris A., Schachner M., Fukuda M., Marth J.D., 2004. Sialyltransferase ST8Sia-II assembles a subset of polysialic acid that directs hippocampal axonal targeting and promotes fear behavior. J. Biol. Chem. 279, 32603-32613. doi: 10.1074/jbc.M403429200.

Angkaw A.C., Ross B.S., Pittman J.O., Kelada A.M., Valencerina M.A., Baker D.G., 2013. Post-traumatic stress disorder, depression, and aggression in OEF/OIF veterans. Mil Med. 178, 1044-1050. doi: 10.7205/MILMED-D-13-00061.

Araki R., Ago Y., Hasebe S., Nishiyama S., Tanaka T., Oka S., 2014. Involvement of prefrontal AMPA receptors in encounter stimulation-induced hyperactivity in isolation-reared mice. Int. J. Neuropsychopharmacol. 17, 883-893. doi: 10.1017/S1461145713001582.

Arnold R.S., Shi J., Murad E., Whalen A.M., Sun C.Q., Polavarapu R., Parthasarathy S., Petros J.A., Lambeth J.D., 2001. Hydrogen peroxide mediates the cell growth and transformation caused by the mitogenic oxidase Nox1. Proc. Natl. Acad. Sci. USA. 98, 5550-5555. doi: 10.1073/pnas.101505898.

Arnsten AFT. Stress signalling pathways that impair prefrontal cortex structure and function. Nat Rev Neurosci. 2009;10:410-422.

Azenha D., Alves M., Matos R., Santa J.F., Silva B., Cordeiro C., Vieira D.N., Ambrósio A.M., 2009. Male specific association between the 5-HTR6 gene 267C/T SNP and suicide in the Portuguese population. Neurosci Lett. 466, 128-130. doi: 10.1016/j.neulet.2009.09.040.

Bazhenova N., Waider J., Bonopartes D., Veniaminova E., Markova N., Costa-Nunes J., Zubkov E., Gorlova A., Pavlov D., Morozova A., Lesch K.P., Strekalova T., 2017. Stress-induced aggression in 
mice and evidence for preventive effects of drugs with proneurogenetic activity. Gottingen Neuroscience Meeting. p. 170.

Been L.E., Moore K.M., Kennedy B.C., Meisel R.L., 2016. Metabotropic Glutamate Receptor and Fragile X Signaling in a Female Model of Escalated Aggression. Biol Psychiatry. 79, 685-692. doi: 10.1016/j.biopsych.2015.07.021.

Benton D., Donohoe R.T., 1999. The effects of nutrients on mood. Public Health Nutr. 3, 403-409. doi: 10.1017/S1368980099000555.

Bettendorff L., Lakaye B., Kohn G., Wins P., 2014. Thiamine triphosphate: a ubiquitous molecule in search of a physiological role. Metab Brain Dis. 29, 1069-1082. doi: 10.1007/s11011-0149509-4.

Beurel, E., Grieco, S.F., Jope, R.S., 2015. Glycogen synthase kinase-3 (GSK3): regulation, actions, and diseases. Pharmacol. Ther. 148, 114-131. doi: 10.1016/j.pharmthera.2014.11.016.

Block M.L., Hong J.S., 2007. Chronic microglial activation and progressive dopaminergic neurotoxicity. Biochem Soc Trans. 35, 1127-1132. doi: 10.1042/BST0351127.

Boufleur N., Antoniazzi C.T., Pase C.S., Benvegnú D.M., Dias V.T., Segat H.J., Roversi K., Roversi K., Nora M.D., Koakoskia G., Rosa J.G., Barcellos L.J., Bürger M.E., 2013. Neonatal handling prevents anxiety-like symptoms in rats exposed to chronic mild stress: behavioral and oxidative parameters. Stress. 16, 321-330. doi: 10.3109/10253890.2012.723075.

Boyko A., Ksenofontov A., Ryabov S., Baratova L., Graf A., Bunik V., 2018. Delayed Influence of Spinal Cord Injury on the Amino Acids of NO - Metabolism in Rat Cerebral Cortex Is Attenuated by Thiamine. Front Med (Lausanne). 2018. 15, 249. doi: 10.3389/fmed.2017.00249.

Bozic I., Savic D., Stevanovic I., Pekovic S., Nedeljkovic N., Lavrnja I., 2015. Benfotiamine upregulates antioxidative system in activated BV-2 microglia cells. Front. Cell. Neurosci. 9: 351. doi: 10.3389/fncel.2015.00351.

Bullock S.P., Rogers L.J., 1986. Glutamate-induced asymmetry in the sexual and aggressive behavior of young chickens. Pharmacol Biochem Behav. 24, 549-554. doi: 10.1016/0091-3057(86)905563.

Bunik V.I., 2013. Thiamin-dependent enzymes: new perspectives from the interface between chemistry and biology. FEBS J. 280, 6373. doi: 10.1111/febs.12589. 
Calandreau L., Márquez C., Bisaz R., Fantin M., Sandi C., 2010. Differential impact of polysialyltransferase ST8SiaII and ST8SiaIV knockout on social interaction and aggression. Genes Brain Behav. 9, 958-967. doi: 10.1111/j.1601-183X.2010.00635.x. Caspi A., McClay J., Moffitt T.E., Mill J., Martin J., Craig I.W., Taylor A., Poulton R., 2002. Role of genotype in the cycle of violence in maltreated children. Science (New York, N.Y.), 297, 851-854. doi:10.1126/science.1072290.

Chrousos G.P., Gold P.W., 1992. The concepts of stress and stress system disorders. Overview of physical and behavioral homeostasis. J. Am. Med. Assoc. 267, 1244-1252. doi: $10.1001 /$ jama.267.9.1244.

Cline B.H., Anthony D.C., Lysko A., Dolgov O., Anokhin K., Schroeter C.A., Malin D., Kubatiev A., Steinbusch H.W., Lesch K.P., Strekalova T., 2015a. Lasting downregulation of the lipid peroxidation enzymes in the prefrontal cortex of mice susceptible to stress-induced anhedonia. Behav Brain Res. 276, 118-129. doi: 10.1016/j.bbr.2014.04.037.

Cline B.H., Costa-Nunes J.P., Cespuglio R., Markova N., Santos A.I., Bukhman Y.V., Kubatiev, A., Steinbusch, H.W., Lesch, K.P., Strekalova, T., 2015b. Dicholine succinate, the neuronal insulin sensitizer, normalizes behavior, REM sleep, hippocampal pGSK3 beta and mRNAs of NMDA receptor subunits in mouse models of depression. Front. Behav. Neurosci. 26, 9-37. doi: 10.3389/fnbeh.2015.00037.

Clark C.R., Galletly C.A., Ash D.J., Moores K.A., Penrose R.A., McFarlane A.C., 2009. Evidence-based medicine evaluation of electrophysiological studies of the anxiety disorders. Clinic EEG Neurosci. 40, 84-112. doi: 10.1177/155005940904000208.

Coccaro E.F., Lee R., Vezinab P., 2013. Cerebrospinal fluid glutamate concentration correlates with impulsive aggression in human subjects. J Psychiatr Res. 47, 1247-1253. doi: 10.1016/j.jpsychires.2013.05.001.

Coccaro E.F., Lee R., Gozal D., 2016. Elevated Plasma Oxidative Stress Markers in Individuals With Intermittent Explosive Disorder and Correlation With Aggression in Humans. Biol Psychiatry. 79, 127-135. doi: 10.1016/j.biopsych.2014.01.014.

Constantini F., D'Amato F., 2006. Ultrasonic vocalization in mice and rats: social contexts and functions. Acta Zool. 52, 619-633.

Costa-Nunes J., Zubareva O., Araújo-Correia M., Valença A., Schroeter C., Pawluski J.L., Vignisse J., Steinbusch H., Hermes D., Phillipines M., Steinbusch H.M., Strekalova T., 2014. Altered 
emotionality, hippocampus-dependent performance and expression of NMDA receptor subunit mRNAs in chronically stressed mice. Stress. 17, 108-116. doi: 10.3109/10253890.2013.872619.

Couch Y., Anthony D.C., Dolgov O., Revischin A., Festoff B., Santos A.I., Steinbusch H.W., Strekalova T., 2013. Microglial activation, increased TNF and SERT expression in the prefrontal cortex define stress-altered behaviour in mice susceptible to anhedonia. Brain Behav. Immun. 29, 136-146. doi: 10.1016/j.bbi.2012.12.017.

Couch Y., Trofimov A., Markova N., Nikolenko V., Steinbusch H.W., Chekhonin V., Schroeter C., Lesch K.P., 2016. Low-dose lipopolysaccharide (LPS) inhibits aggressive and augments depressive behaviours in a chronic mild stress model in mice. $\mathrm{J}$ Neuroinflammation. 13, 1-8. doi: 10.1186/s12974-016-0572-0.

Daniels W.M., Marais L., Stein D.J., Russell V.A., 2012. Exercise normalizes altered expression of proteins in the ventral hippocampus of rats subjected to maternal separation. Exp Physiol. 97, 239-247. doi: 10.1113/expphysiol.2011.061176.

Diehl L.A., Alvares L.O., Noschang C., Engelke D., Andreazza A.C., Gonçalves C.A., Quillfeldt J.A., Dalmaz C., 2012. Long-lasting effects of maternal separation on an animal model of posttraumatic stress disorder: effects on memory and hippocampal oxidative stress. Neurochem Res. 37, 700-707. doi: 10.1007/s11064-011-0660-6.

Ebuehi O.A., Ayinde O.C. Neurobehavioural and neurotoxic effects of L-ascorbic acid and L-tryptophan in lead exposed rats. Nig Q J Hosp Med. 2012 Oct-Dec;22(4):240-4.

El-Tarras Ael-S., Attia H.F., Soliman M.M., El Awady M.A., Amin A.A., 2016. Neuroprotective effect of grape seed extract against cadmium toxicity in male albino rats. Int $\mathrm{J}$ Immunopathol Pharmacol. 29, 398-407. doi: 10.1177/0394632016651447.

Fan D., Grooms S.Y., Araneda R.C., Johnson A.B., Dobrenis K., Kessler J.A., Zukin R.S., 1999. AMPA receptor protein expression and function in astrocytes cultured from hippocampus. J Neurosci Res. 57, 557-571.

Fanning J.R., Lee R., Gozal D., Coussons-Read M., Coccaro E.F., 2015. Childhood trauma and parental style: Relationship with markers of inflammation, oxidative stress, and aggression in healthy and personality disordered subjects. Biol Psychol. 112, 56-65. doi: 10.1016/j.biopsycho.2015.09.003. 
Fanning J.R., Keedy S., Berman M.E., Lee R., Coccaro E.F., 2017. Neural Correlates of Aggressive Behavior in Real Time: a Review of fMRI Studies of Laboratory Reactive Aggression. Curr Behav Neurosci Rep. 2, 138-150. doi: 10.1007/s40473-017-01158.

Fontes M.A., Xavier C.H., Marins F.R., Limborço-Filho M., Vaz G.C., Müller-Ribeiro F.C., Nalivaiko E., 2014. Emotional stress and sympathetic activity: Contribution of dorsomedial hypothalamus to cardiac arrhythmias. Brain Research. 1554, 49-58. doi: 10.1016/j.brainres.2014.01.043.

Garcia-Alloza M., Hirst W.D., Chen C.P., Lasheras B., Francis P.T., Ramírez M.J., 2004. Differential involvement of 5-HT(1B/1D) and 5-HT6 receptors in cognitive and non-cognitive symptoms in Alzheimer's disease. Neuropsychopharmacology. 29, 410-416. doi: 10.1038/sj.npp.1300330.

Ghaleiha A., Davari H., Jahangard L., Haghighi M., Ahmadpanah M., Seifrabie M.A., Bajoghli H., Holsboer-Trachsler E., Brand S., 2016. Adjuvant thiamine improved standard treatment in patients with major depressive disorder: results from a randomized, double-blind, and placebo-controlled clinical trial. Eur Arch Psychiatry Clin Neurosci. 266, 695-702. doi: 10.1007/s00406-016-0685-6.

Gvozdjáková A., Kucharská J., Ostatníková D., Babinská K., Nakládal D., Crane F.L., 2014. Ubiquinol improves symptoms in children with autism. Oxid Med Cell Longev. 2014;2014:798957. doi: 10.1155/2014/798957.

Haller J., Mikics E., Halász J., Tóth M., 2005. Mechanisms differentiating normal from abnormal aggression: glucocorticoids and serotonin. Eur J Pharmacol. 526, 89-100. doi: 10.1016/j.ejphar.2005.09.064.

Hamazaki T, Sawazaki S, Itomura M, Asaoka E, Nagao Y, Nishimura N, Yazawa K, Kuwamori T, Kobayashi M.The effect of docosahexaenoic acid on aggression in young adults. A placebocontrolled double-blind study. J Clin Invest. 1996 ,97 1129-1133.

Henley J.M., Wilkinson K.A., 2013. Dialogues AMPA receptor trafficking and the mechanisms underlying synaptic plasticity and cognitive aging. Clin Neurosci. 15, 11-27.

Hollmann M., O'Shea-Greenfield A., Rogers S.W., Heinemann S., 1989. Cloning by functional expression of a member of the glutamate receptor family. Nature. 342, 643-668. doi: 10.1038/342643a0. 
Hume R.I., Dingledine R., Heinemann S.F., 1991. Identification of a site in glutamate receptor subunits that controls calcium permeability. Science. 253, 1028-1031. doi: 10/1126/science.1653450.

Keinänen K., Wisden W., Sommer B., Werner P., Herb A., Verdoorn T.A., Sakmann B., Seeburg P.H., 1990. A family of AMPAselective glutamate receptors. Science. 249, 556-560. doi: 10.1126/science.2166337.

Kessler R.C., Sampson N.A., Berglund P., Gruber M.J., Al-Hamzawi A., Andrade L., Bunting B., Demyttenaere K., Florescu S., de Girolamo G., Gureje O., He Y., Hu C., Huang Y., Karam E., Kovess-Masfety V., Lee S., Levinson D., Medina Mora M.E., Moskalewicz J., Nakamura Y., Navarro-Mateu F., Browne M.A., Piazza M., Posada-Villa J., Slade T., Ten Have M., Torres Y., Vilagut G., Xavier M., Zarkov Z., Shahly V., Wilcox M.A., 2015. Anxious and non-anxious major depressive disorder in the World Health Organization World Mental Health Surveys. Epidemiol Psychiatr Sci. 24, 210-226. doi: 10.1017/S2045796015000189.

Khlghatyan J.1., Evstratova A., Chamberland S., Marakhovskaia A., Bahremand A., Toth K., Beaulieu J.M., 2018. Mental IllnessesAssociated Fxr1 and Its Negative Regulator Gsk3B Are Modulators of Anxiety and Glutamatergic Neurotransmission. Front Mol Neurosci. 12, 11-119. doi: 10.3389/fnmol.2018.00119.

Kim J.J., Haller J., 2007. Glucocorticoid hyper- and hypofunction: stress effects on cognition and aggression. Ann N Y Acad Sci. 1113, 291 303. doi: 10.1196/annals.1391.014.

Kuloglu M., Atmaca M., Tezcan E., Gecici O., Ustundag B., Bulut S., 2002. Antioxidant enzyme and malondialdehyde levels in patients with panic disorder. Neuropsychobiology. 46, 186-189. doi: $10.1159 / 000067810$.

Lee S.H., Valtschanoff J.G., Kharazia V.N., Weinberg R., Sheng M., 2001. Biochemical and morphological characterization of an intracellular membrane compartment containing AMPA receptors. Neuropharmacology. 41, 680-692. doi: 10.1016/S0028-3908(01)00124-1.

Lento W., Ito T., Zhao C., Harris J.R., Huang W., Jiang C., Owzar K., Piryani S. Racioppi L. Chao N., Reyal T., 2014. Loss of B-catenin triggers oxidative stress and impairs hematopoietic regeneration. Genes Dev. 28, 995-1004. doi: 10.1101/gad.231944.113.

Leroy F., Park J., Asok A., Brann D.H., Meira T., Boyle L.M., Buss E.W., Kandel E.R., Siegelbaum S.A., 2018. A circuit from hippocampal 
CA2 to lateral septum disinhibits social aggression. Nature. 9, 4163. doi: 10.1038/s41467-018-06501-w.

Lesch K.P., Araragi N., Waider J., van den Hove D., Gutknecht L., 2012. Targeting brain serotonin synthesis: insights into neurodevelopmental disorders with long-term outcomes related to negative emotionality, aggression and antisocial behaviour. Philos Trans R Soc Lond B Biol Sci. 367, 2426-2443. doi: 10.1098/rstb.2012.0039.

Machado C.J., Bachevalier J., 2006. The impact of selective amygdala, orbital frontal cortex, or hippocampal formation lesions on established social relationships in rhesus monkeys (Macaca mulatta). Behavioral Neuroscience. 120, 761-786. doi: 10.1037/0735-7044.120.4.761.

Malatynska E., Steinbusch H.W., Redkozubova O., Bolkunov A., Kubatiev A., Yeritsyan N.B., Vignisse J., Bachurin S., Strekalova T., 2012. Anhedonic-like traits and lack of affective deficits in 18-month-old C57BL/6 mice: implications for modeling elderly depression. Exp. Gerontol. 47, 552-564. doi: 10.1016/j.exger.2012.04.010.

Malki K., Tosto M.G., Pain O., Sluyter F., Mineur Y.S., Crusio W.E., de Boer S., Sandnabba K.N., Kesserwani J., Robinson E., Schalkwyk L.C., Asherson P., 2016. Comparative mRNA analysis of behavioral and genetic mouse models of aggression. Am J Med Genet B Neuropsychiatr Genet. 171, 427-436. doi: 10.1002/ajmg.b.32424.

Marais L., Stein D.J., Daniels W.M., 2009. Exercise increases BDNF levels in the striatum and decreases depressive-like behavior in chronically stressed rats. Metab Brain Dis. 24, 587-597. doi: 10.1007/s11011-009-9157-2.

Marcos B., García-Alloza M., Gil-Bea F.J., Chuang T.T., Francis P.T., Chen C.P., Tsang S.W., Lai M.K., Ramirez M.J., 2008. Involvement of an altered 5-HT-6 receptor function in behavioral symptoms of Alzheimer's disease. J Alzheimers Dis. 14, 43-50. doi: 10.3233/JAD-2008-14104.

Markova N., Bazhenova N., Anthony D.C., Vignisse J., Svistunov A., Lesch K.P., Bettendorff L., Strekalova T., 2017. Thiamine and benfotiamine improve cognition and ameliorate GSK-3Bassociated stress-induced behaviours in mice. Prog Neuropsychopharmacol Biol Psychiatry. 75, 148-156. doi: 10.1016/j.pnpbp.2016.11.001. 
Meneses A., 2017. Neural activity, memory, and dementias: serotonergic markers. Behav Pharmacol. 28, 132-141. doi: 10.1097/FBP.0000000000000279.

Mkrtchyan G., Aleshin V., Parkhomenko Y., Kaehne T., Di Salvo M.L., Parroni A., Contestabile R., Vovk A., Bettendorff L., Bunik V., 2015. Molecular mechanisms of the non-coenzyme action of thiamin in brain: biochemical, structural and pathway analysis. Sci Rep. 5: 12583. doi: 10.1038/srep12583.

Mkrtchyan G.V., Üçal M., Müllebner A., Dumitrescu S., Kames M., Moldzio R., Molcanyi M., Schaefer S., Weidinger A., Schaefer U., Hescheler J., Duvigneau J.C., Redl H., Bunik V.I., Kozlov A.V., 2018. Thiamine preserves mitochondrial function in a rat model of traumatic brain injury, preventing inactivation of the 2oxoglutarate dehydrogenase complex. Biochim Biophys Acta. 1859, 925-931. doi: 10.1016/j.bbabio.2018.05.005.

Morozova A., Zubkov E., Strekalova T., Kekelidze Z., Storozeva Z., Schroeter C.A., Bazhenova N., Lesch K.P., Cline B.H., Chekhonin V., 2016. Ultrasound of alternating frequencies and variable emotional impact evokes depressive syndrome in mice and rats. Prog Neuro-Psychopharmacol Biol Psychiatry. 68, 5263. doi: 10.1016/j.pnpbp.2016.03.003.

Muñoz-Blanco J., Yusta B., Cordoba F., 1986. Differential distribution of neurotransmitter amino acids from the limbic system of aggressive and non-aggressive bull strains. Pharmacol Biochem Behav. 25, 71-75. https://doi.org/10.1016/0091-3057(86)90232-7.

Newcombe J., Uddin A., Dove R., Patel B., Turski L., Nishizawa Y., Smith T., 2008. Glutamate receptor expression in multiple sclerosis lesions. Brain Pathol. 18, 52-61. doi: 10.1111/j.17503639.2007.00101.x

Ng F., Berk M., Dean O., Bush A.I., 2008. Oxidative stress in psychiatric disorders: Evidence base and therapeutic implications. Int $\mathrm{J}$ Neuropsychopharmacol. 15, 1-26. doi: 10.1017/S1461145707008401.

Okazawa H., Ikawa M., Tsujikawa T., Kiyono Y., Yoneda M., 2014. Brain imaging for oxidative stress and mitochondrial dysfunction in neurodegenerative diseases. Q J Nucl Med Mol Imaging. 58, 387-397. doi: 10.1016/j.jns.2017.08.741.

Pan X., Gong N., Zhao J., Yu Z., Gu F., Chen J., Sun X., Zhao L., Yu M., Xu Z., Dong W., Qin Y., Fei G., Zhong C., Xu T.L., 2010. Powerful beneficial effects of benfotiamine on cognitive impairment and beta-amyloid deposition in amyloid precursor protein/presenilin- 
1 transgenic mice. Brain. 133, 1342-1351. doi: 10.1093/brain/awq069.

Pan X., Chen Z., Fei G., Pan S., Bao W., Ren S., Guan Y., Zhong C. LongTerm Cognitive Improvement After Benfotiamine Administration in Patients with Alzheimer's Disease., 2016. Neurosci Bull. 32, 591-596. doi:10.1007/s12264-016-0067-0.

Partyka A., Wasik A., Jastrzębska-Więsek M., Mierzejewski P., Bieńkowski P., Kołaczkowski M., Wesołowska A., 2016. ADN1184, a monoaminergic ligand with 5-HT6/7 receptor antagonist action, exhibits activity in animal models of anxiety. Naunyn Schmiedebergs Arch Pharmacol. 389, 593-602. doi: 10.1007/s00210-016-1229-3.

Pavlov D., Markova N., Bettendorff L., Chekhonin V., Pomytkin I., Lioudyno V., Svistunov A., Ponomarev E., Lesch K.P., Strekalova T., 2017. Elucidating the functions of brain GSK3a: Possible synergy with GSK3B upregulation and reversal by antidepressant treatment in a mouse model of depressive-like behaviour. Behav Brain Res. 335, 122-127. doi: 10.1016/j.bbr.2017.08.018.

Pavlov D., Bettendorff L., Gorlova A., Olkhovik A., Kalueff A.V., Ponomarev E.D., Inozemtsev A., Chekhonin V., Lesch K.P., Anthony D.C., Strekalova T., 2018. Neuroinflammation and aberrant hippocampal plasticity in a mouse model of emotional stress evoked by exposure to ultrasound of alternating frequencies. Prog Neuropsychopharmacol Biol Psychiatry. 90, 104-116. doi: 10.1016/j.pnpbp.2018.11.014.

Patki G., Solanki N., Atrooz F., Allam F., Salim S., 2013. Depression, anxiety-like behavior and memory impairment are associated with increased oxidative stress and inflammation in a rat model of social stress. Brain research. 1539, 73-86. doi: 10.1016/j.brainres.2013.09.033.

Patki G., Atrooz F., Alkadhi I., Solanki N., Salim S., 2014. High aggression in rats is associated with elevated stress, anxiety-like behavior, and altered catecholamine content in the brain. 584, 308-313. doi: 10.1016/j.neulet.2014.10.051.

Pereira M., Martynhak B.J., Andreatini R., Svenningsson P., 2015. 5HT6 receptor agonism facilitates emotional learning. Front Pharmacol. 16, 6-200. doi: 10.3389/fphar.2015.00200.

Rada P., Rojo A.I., Evrard-Todeschi N., Innamorato N.G., Cotte A., Jaworski T., Tobón-Velasco J.C., Devijver H., García-Mayoral M.F., Van Leuven F., 2012. Structural and functional characterization of $\mathrm{Nrf} 2$ degradation by the glycogen synthase 
kinase 3/6-TrCP axis. Mol. Cell Biol. 32, 3486-3499. doi: 10.1128/MCB.00180-12.

Raj V., Ojha S., Howarth F.C., Belur P.D., Subramanya S.B., 2018. Therapeutic potential of benfotiamine and its molecular targets. Eur Rev Med Pharmacol Sci. 22, 3261-3273. doi: 10.26355/eurrev_201805_15089.

Rammal H., Bouayed J., Soulimani R., 2010. A direct relationship between aggressive behavior in the resident/intruder test and cell oxidative status in adult male mice. Eur J Pharmacol. 627, 173-176. doi: 10.1016/j.ejphar.2009.11.001.

Ramirez M.J., 2013. 5-HT6 receptors and Alzheimer's disease. Alzheimers Res Ther. 5, 15. doi: 10.1186/alzrt169.

Roy A., Jana A., Yatish K., Freidt M.B., Fung Y.K., Martinson J.A., Pahan K., 2008. Reactive oxygen species up-regulate CD11b in microglia via nitric oxide: Implications for neurodegenerative diseases. Free Radic Biol Med. 45, 686-699. doi: 10.1016/j.freeradbiomed.2008.05.026.

Roy A., Oldehinkel A.J., Verhulst F.C., Ormel J., Hartman C.A., 2014. Anxiety and disruptive behavior mediate pathways from attention-deficit/hyperactivity disorder to depression. J Clin Psychiatry. 75, 108-113. doi: 10.4088/JCP.13m08648.

Salminen L.E., Paul R.H., 2014. Oxidative stress and genetic markers of suboptimal antioxidant defense in the aging brain: a theoretical review. Rev Neurosci. 25, 805-819. doi: 10.1515/revneuro-2014-0046.

Sanchez-Ramirez G.M., Caram-Salas N.L., Rocha-Gonzalez H.I., VidalCantu G.C., Medina-Santillan R., Reyes-Garcia G., 2006. Benfotiamine relieves inflammatory and neuropathic pain in rats. European journal of pharmacology. 530, 48-53. doi: 10.1016/j.ejphar.2005.11.016.

Schiavone S., Colaianna M., Curtis L., 2015. Impact of early life stress on the pathogenesis of mental disorders: relation to brain oxidative stress. Curr Pharm Des. 21, 1404-1412. doi: 10.2174/1381612821666150105143358.

Schmid U., Stopper H., Heidland A., Schupp N., 2008. Benfotiamine exhibits direct antioxidative capacity and prevents induction of DNA damage in vitro. Diabetes Metab. Res. 24, 371-377.

Selye, H., 1974. Stress without Distress. J.B. Lippincott Company, Philadelphia, 171.

Shimizu K., Kurosawa N., Seki K., 2016. The role of the AMPA receptor and 5-HT(3) receptor on aggressive behavior and depressive-like 
symptoms in chronic social isolation-reared mice. Physiol Behav. 153, 70-83. doi: 10.1016/j.physbeh.2015.10.026.

Shimshek D.R., Bus T., Grinevich V., Single F.N., Mack V., Sprengel R., Spergel D.J., Seeburg P.H., 2006. Impaired reproductive behavior by lack of GluR-B containing AMPA receptors but not of NMDA receptors in hypothalamic and septal neurons. Mol Endocrinol. 20, 219-231. doi: 10.1210/me.2005-0262.

Shoeb M., Ramana K.V., 2012. Anti-inflammatory effects of benfotiamine are mediated through the regulation of the arachidonic acid pathway in macrophages. Free Radic Biol Med. 52, 182-190. doi: 10.1016/j.freeradbiomed.2011.10.444.

Siever L.J., 2008. Neurobiology of aggression and violence. Am J Psychiatry. 165, 429-442. doi: 10.1176/appi.ajp.2008.07111774.

Simonov P.V., 1997. Brain mechanisms of emotions. Neurosci Behav Physiol. 27, 405-413. doi: 10.1007/BF02462942.

Singh G.K., Garabadu D., Muruganandam A.V., Joshi V.K., Krishnamurthy S., 2009. Antidepressant activity of Asparagus racemosus in rodent models. Pharmacol Biochem Behav. 91, 283-290. doi: 10.1016/j.pbb.2008.07.010.

Solanki N., Atrooz F., Asghar S., Salim S., 2016. Tempol protects sleepdeprivation induced behavioral deficits in aggressive male LongEvans rats. Neurosci Lett. 612, 245-250. doi: 10.1016/j.neulet.2015.12.032.

Song I., Huganir R.L., 2002. Regulation of AMPA receptors during synaptic plasticity. Trends Neurosci. 25, 578-588. doi: 10.1016/S0166-2236(02)02270-1.

Strekalova T., Steinbusch H., 2010. Measuring behavior with chronic stress depression model in mice. Progr Neuro-Psychopharm Biol Psych. 34, 348-361. doi: 10.1016/j.pnpbp.2009.12.014.

Strekalova T., Evans M., Chernopiatko A., Couch Y., Costa-Nunes J., Cespuglio R., Chesson L., Vignisse J., Steinbusch H.W., Anthony D.C., Pomytkin I., Lesch K.P., 2015. Deuterium content of water increases depression susceptibility: the potential role of a serotonin-related mechanism. Behav Brain Res. 277, 237-244. doi: 10.1016/j.bbr.2014.07.039.

Strekalova T., Bahzenova N., Trofimov A., Schmitt-Böhrer A.G., Markova N., Grigoriev V., Zamoyski V., Serkova T., Redkozubova O., Vinogradova D., Umriukhin A., Fisenko V., Lillesaar C., Shevtsova E., Sokolov V., Aksinenko A., Lesch k.P., Bachurin S., 2018. Pro-neurogenic, Memory-Enhancing and Anti-stress Effects of DF302, a Novel Fluorine Gamma- 
Carboline Derivative with Multi-target Mechanism of Action. Mol Neurobiol. 55, 335-349. doi: 10.1007/s12035-017-0745-6.

Stutzman D and Dopheide J. Acetylcysteine for treatment of autism spectrum disorder symptoms. Am J Health Syst Pharm. 2015 Nov 15;72(22):1956-9. doi: 10.2146/ajhp150072.

Sun X., Li X., Pan R., Xu Y., Wang Q., Song M., 2018. Total Saikosaponins of Bupleurum yinchowense reduces depressive, anxiety-like behavior and increases synaptic proteins expression in chronic corticosterone-treated mice. BMC Complement Altern Med. 18, 117. doi: 10.1186/s12906-018-2186-9.

Tapias V., Jainuddin S., Ahuja M., Stack C., Elipenahli C., Vignisse J., Gerges M., Starkova N., Xu H., Starkov A.A., Bettendorff L., Hushpulian D.M., Smirnova N.A., Gazaryan I.G., Kaidery N.A., Wakade S., Calingasan N.Y., Thomas B., Gibson G.E., Dumont M., Beal M.F.. 2018. Benfotiamine treatment activates the Nrf2/ARE pathway and is neuroprotective in a transgenic mouse model of tauopathy. Hum Mol Genet. 27, 2874-2892. doi: $10.1093 / \mathrm{hmg} / \mathrm{ddy} 201$.

Toyoda H., Zhao M.G., Ulzhöfer B., Wu L.J., Xu H., Seeburg P.H., Sprengel R., Kuner R., Zhuo M., 2009. Roles of the AMPA receptor subunit GluA1 but not GluA2 in synaptic potentiation and activation of ERK in the anterior cingulate cortex. Mol Pain. 5:46. doi: 10.1186/1744-8069-5-46.

Tsai S.J., Chiu H.J., Wang Y.C., Hong C.J., 1999. Association study of serotonin-6 receptor variant (C267T) with schizophrenia and aggressive behavior. Neurosci Lett. 271, 135-137. doi: 10.1016/S0304-3940(99)00542-X.

Upton N., Chuang T.T., Hunter A.J., Virley D.J., 2008. 5-HT(6) receptor antagonists as novel cognitive enhancing agents for Alzheimer's disease. Neurotherapeutics. 5, 458-469. doi: 10.1016/j.nurt.2008.05.008.

Vekovischeva O.Y., Aitta-Aho T., Echenko O., Kankaanpää A., Seppälä T., Honkanen A., Sprengel R., Korpi E.R., 2004. Reduced aggression in AMPA-type glutamate receptor GluR-A subunitdeficient mice. Genes Brain Behav. 3, 253-265. doi: 10.1111/j.1601-1848.2004.00075.x.

Vekovischeva O.Y., Aitta-aho T., Verbitskaya E., Sandnabba K., Korpi E.R., 2007. Acute effects of AMPA-type glutamate receptor antagonists on intermale social behavior in two mouse lines bidirectionally selected for offensive aggression. Pharmacol Biochem Behav. 87, 241-249. doi: 10.1016/j.pbb.2007.04.020. 
Verona E., Kilmer A., 2007. Stress exposure and affective modulation of aggressive behavior in men and women. Journal of abnormal psychology. 2007. 116, 410-421. doi: 10.1037/0021843X.116.2.410.

Vignisse J., Steinbusch H.W.M., Bolkunov A., Nunes J., Santos A.I., Grandfils C., Bachurin S., Strekalova T., 2011. Dimebon enhances hippocampus-dependent learning in both appetitive and inhibitory memory tasks in mice. Progr Neuro-Psychopharm Biol Psych. 35, 510-522. doi: 10.1016/j.pnpbp.2010.12.007.

Vignisse J., Sambon M., Gorlova A., Pavlov D., Caron N., Malgrange B., Shevtsova E., Svistunov A., Anthony D., Markova N., Bazhenova N., Coumans B., Lakaye B., Wins P., Strekalova T., Bettendorff L., 2017. Thiamine and benfotiamine prevent stress-induced suppression of hippocampal neurogenesis in mice exposed to predation without affecting brain thiamine diphosphate levels. Molecular and Cellular Neuroscience. 82, 126-136. doi: 10.1016/j.men.2017.05.005.

Veenema A.H., Neumann I.D., 2007. Neurobiological mechanisms of aggression and stress coping: a comparative study in mouse and rat selection lines. Brain Behav Evol. 70, 274-285. doi:10.1159/000105491.

Volvert M.L., Seyen S., Piette M., Evrard B., Gangolf M., Plumier J.C., Bettendorff L., 2008. Benfotiamine, a synthetic S-acyl thiamine derivative, has different mechanisms of action and a different pharmacological profile than lipid-soluble thiamine disulfide derivatives. BMC Pharmacol. 8, 10. doi: 10.1186/1471-2210-8-10.

Winkler D., Daher F., Wüstefeld L., Hammerschmidt K., Poggi G., Seelbach A., Krueger-Burg D., Vafadari B., Ronnenberg A., Liu Y., Kaczmarek L., Schlüter O.M., Ehrenreich H., Dere E., 2017. Hypersocial behavior and biological redundancy in mice with reduced expression of PSD95 or PSD93. Behav Brain Res. 352, 35-45. doi: 10.1016/j.bbr.2017.02.011.

Yun H.M., Rhim H., 2011. The serotonin-6 receptor as a novel therapeutic target. Exp Neurobiol. 20, 159-168. doi: 10.5607/en.2011.20.4.159.

Zhang Y. Cudmore R.H., Lin D.T., Linden D.J., Huganir R.L., 2015. Visualization of NMDA receptor-dependent AMPA receptor synaptic plasticity in vivo. Nat Neurosci. 18, 402-407. doi: 10.1038/nn.3936.

Zhang X., He X., Chen Q., Lu J., Rapposelli S., Pi R., 2018. A review on the hybrids of hydroxycinnamic acid as multi-target-directed 
ligands against Alzheimer's disease. Bioorg Med Chem. 26, 543550. doi: 10.1016/j.bmc.2017.12.042.

\section{Supplementary material}

\section{Ultrasound radiation}

Ultrasound radiation of average intensity of $50 \pm 5 \mathrm{~dB}$ and variable frequencies in a $20-45 \mathrm{~Hz}$ range was constantly delivered in a quiet laboratory environment to the experimental groups of mice using a random schedule of alternating frequencies via a manufactured device (Weitech, Wavre, Belgium). The range of ultrasound stimulation frequency was alternated every $10 \mathrm{~min}$ between the following settings: low frequencies $(20-25 \mathrm{kHz})$, middle range frequencies $(>25<40 \mathrm{kHz})$ and high range frequencies $(40-45 \mathrm{kHz})$. During the 10-min periods, ultrasound frequencies fluctuated at short time spans of $\leq 1 \mathrm{~s} .35 \%$ of the emission time consisted of $20-25$ and $25-40 \mathrm{kHz}$ frequencies, and $30 \%$ of the emission time was consisted of $40-45 \mathrm{kHz}$. The ultrasound device was hung $2 \mathrm{~m}$ above the cages of the experimental groups with an average horizontal distance to cages of $2.5 \mathrm{~m}$. The position of the cages, with respect to the stimulator, was changed weekly. The selectivity of the adverse effects of lowfrequency ultrasound during the radiation period versus the potential general negative effects of a constant noise accompanying the procedure described here was demonstrated previously (Morozova et al., 2016). The intensity of the sound 
during above-indicated intervals was fluctuating with the range $\pm 10 \%$ of the averaged value, i.e., $\pm 5 \mathrm{~dB}$. The shape of the ultrasound signals was fluctuating that mimics natural ultrasonic vocalizations of rodents is given below.

\section{A study of impact of range of ultrasound frequency} stimulation on mouse emotional behaviour

We explored the specificity of effects of "emotionally meaningful" ultrasound within the low frequency ranges, in particular, in regard to the induction of behavioural effects in mice. In this study, immediate behavioural effects of ultrasound at frequency ranges of $16 \mathrm{kHz}, 22-25 \mathrm{kHz}$, and $50-60 \mathrm{kHz}$ on $\mathrm{BALB} / \mathrm{c}$ mice were compared (six mice per group were used). Previously established tests for emotionality and exploration were employed (Vignisse et al., 2014; Strekalova et al., 2013; Veniaminova et al., 2016). 15 min-ultrasound of an average intensity of $50 \mathrm{~dB}$ of the lowest frequency, but not of other frequencies, significantly increased number of freezing episodes ( $<<0.05$ and $p>0.05$, respectively, one-way ANOVA and Tukey test, Fig.1A). Ultrasound at the range of $22-25 \mathrm{~Hz}$ significantly decreased the latency of burying behaviour $(p<0.05$, one-way ANOVA and Tukey test, Fig.1B), number of approaches to palatable food ( $<<0.05$, one-way ANOVA and Tukey test, Fig.1C) and increased the latency to approach a new object $(p<0.05$, oneway ANOVA and Tukey test, Fig.1D), as compared to controls, suggesting anxiogenic-like effects of the applied range of 
ultrasound. Thus, low frequency ultrasound produces negative effects on emotional behavior in BALB/c mice.

\section{Behavioural effects of the ultrasound of selective} ranges of frequencies
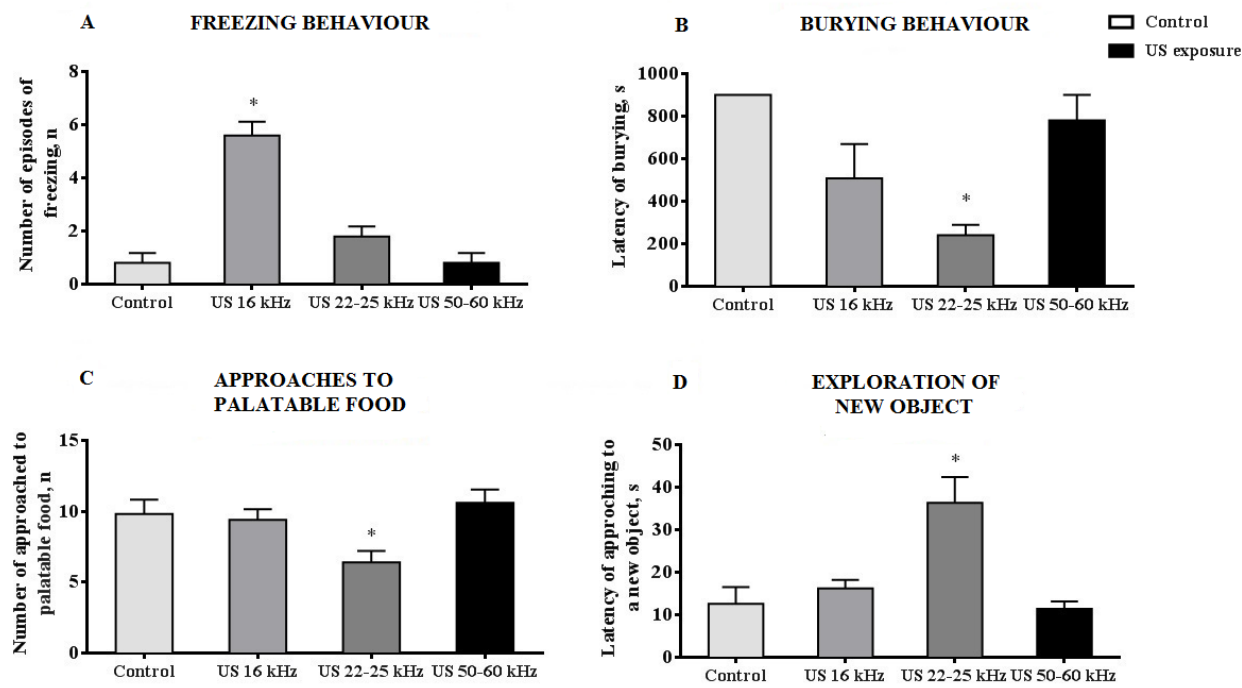

Supplemental Figure 1: Ultrasound of selective ranges of frequencies evokes immediate behavioural effects in BALB/c mice. (A) Mice exposed to a $16 \mathrm{kHz}$-ultrasound displayed increased number of freezing episodes. Mice subjected to $22-25 \mathrm{kHz}$ ultrasound exposure showed (B) reduced latency of burying behaviour, (C) decreased number of approached to palatable food and (D) elevated latency to approach a new object. Thus, low frequency ultrasound produces negative effects on emotional behavior of mice while ultrasound of frequencies of $50-60 \mathrm{kHz}$ did not affect any of measured behavioural parameters. ${ }^{*} \mathrm{p}<0.05$ vs. control group, one-way ANOVA and post-hoc Tukey test. Bars are means \pm SEMs. US - ultrasound exposure. 


\section{Effects of a 1-week, 2-week, or 3-week 50dB}

ultrasound of variable frequencies

In further preliminary experiments, BALB/c mice were subjected to the same housing and experimental conditions as described in the main text, except that the ultrasound exposure lasted 1 week, 2 weeks or 3 weeks. Thereafter, the emotional behaviours of the control and ultrasound-exposed mice were investigated as described elsewhere (Pavlov et al., 2019). Serum corticosterone concentrations were studied as well as described elsewhere (Couch et al., 2013). Briefly, blood was collected transcardially at sacrifice during daytime and centrifuged (10 000 rpm, $15 \mathrm{~min}, 4^{\circ} \mathrm{C}$ ); plasma was collected and stored at $-80^{\circ} \mathrm{C}$ until use. ELISA kit (Sigma-Aldrich, St-Louis, MO, USA) was used for corticosterone assay according to manufacturer's instructions. All samples were run in duplicate. The average intra- and inter-assay variation for all corticosterone assays was below $10 \%$. Six to nine mice per group were used.

Mice exposed to a 1-week or 2-week ultrasound of variable frequencies showed no changes in the duration of floating behaviour in the forced swim test, as well as in sucrose preference and serum corticosterone concentrations, in comparison to control groups ( $p>0.05$, one-way ANOVA and Tukey test, Fig.2 A-C). In comparison to controls, a 3-week ultrasound exposure resulted in significant increase of total time spent with floating, significant decrease of sucrose preference and increase of serum corticosterone concentration ( $<<0.05$ ANOVA and Tukey test). 

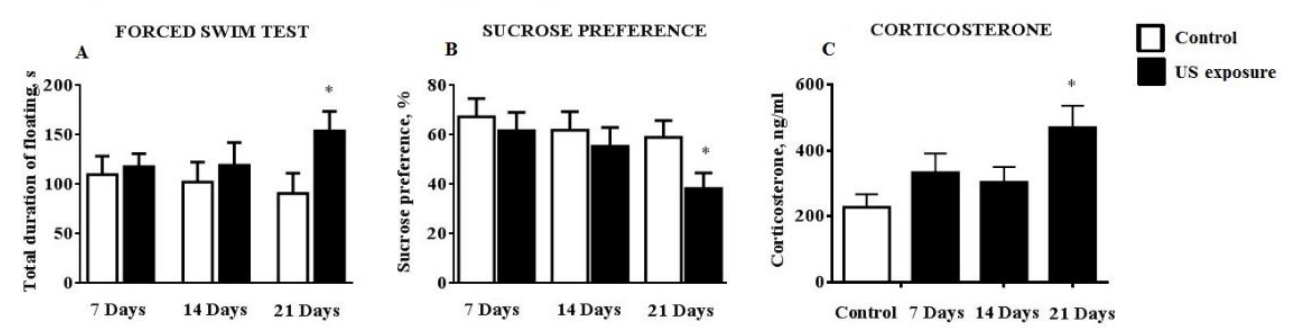

Supplemental Figure 2. Behavioural effects of 1-week, 2week, or 3-week exposures to a $50 \mathrm{~dB}$ ultrasound of variable frequencies. In comparison to control groups, animals subjected to a 3 -week $50 \mathrm{~dB}$ ultrasound of $20-45 \mathrm{~Hz}$ frequencies, but not to 1 -week or 2 week exposures displayed (A) increased total time spent floating, (B) decreased sucrose preference and (C) elevated serum corticosterone concentrations ( ${ }^{*} p<0.05$ vs. control group, one-way ANOVA and posthoc Tukey test). Bars are means \pm SEMs; US - ultrasound exposure.

\section{Effects of buspirone on behaviour following 3 weeks} of ultrasound exposure

In another validation study, male $\mathrm{BALB} / \mathrm{c}$ mice were subjected to the same 3-week ultrasound as described in the main text, and studied, alone with naïve controls, in the elevated $\mathrm{O}$ maze test. In this experiment, ultrasound-exposed mice displayed significantly fewer exits to open arms and time spent therein, as compared with naïve animals, suggesting increased anxiety-like behaviour in the ultrasound-exposed animals $(\mathrm{p}<0.05, t$-test, Fig. 3A,B).

In a separate study, male BALB/c mice were subjected to the same 3-week ultrasound as described in the main text, and received either daily i.p. injections of buspirone, atypical 
antidepressant with anxyolitic properties (Howland, 2015), at the dose $2 \mathrm{mg} / \mathrm{kg}$, or vehicle. Control groups received either of these treatments as well. Ultrasound-exposed vehicle-treated mice had shorter latency to immobility and longer duration of immobility than respective control group, in the tail suspension model $(p<0.05$, two-way ANOVA and Tukey test, Fig.3 D,E). There was a significant decrease of time spent in the open arms of the elevated plus maze and in the center of the open field and increased number of attacks in the social interaction test in the ultrasound-exposed vehicle-treated mice in comparison with vehicle-treated control animals $(\mathrm{p}<0.05$, two-way ANOVA and Tukey test, Fig.3C,E,F). These behavioural differences were not found between buspirone-treated ultrasound-exposed and control groups ( $\mathrm{p}>0.05$, two-way ANOVA and Tukey test, Fig. 3 D,E). Thus, the antidepressant and anti-anxiety treatment with buspirone has counteracted the changes in emotional behaviour during ultrasound exposure of alternating frequencies. In these studies, six to eight mice per group were used, behavioural assays were carried out as described elsewhere (Vignisse et al., 2014; Costa-Nunes et al., 2015; Veniaminova et al., 2016). 

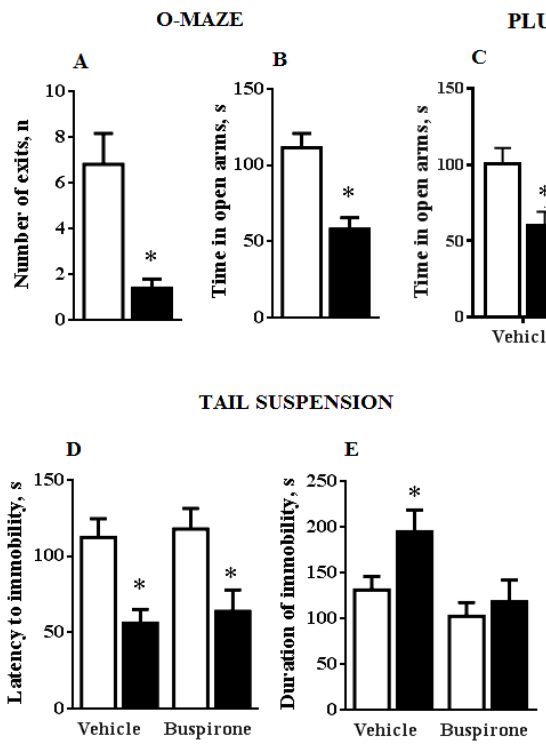

PLUS MAZE

AIL SUSPENSION

OPEN FIELD

SOCIAL INTERACTION
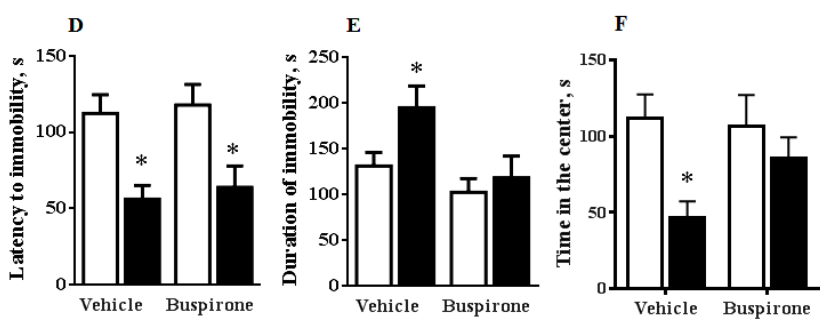

G

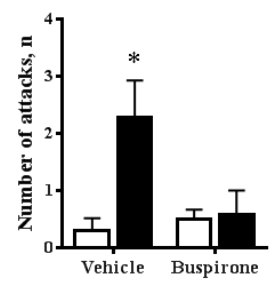

Supplemental Figure 3. Ultrasound-induced changes in anxiety-like and other emotional behaviours are counteracted by the administration of buspirone. In the elevated O-maze, in comparison with control group, ultrasound-exposed mice displayed (A) decreased number of exits and (B) decreased time spent in open arms $\left({ }^{*} \mathrm{p}<0.05\right.$ vs. control, $\mathrm{p}<0.05$, t-test. In the study with chronic buspirone administration, ultrasound-induced (C) decrease of time spent in open arms of the elevated plus maze, (D) decrease of the latency to immobility and (E) increase of duration of immobility in the tail suspension test, as well as (F) decrease of time spent in the center of the open field and $(\mathbf{G})$ increase of number of attacks in a residentintruder test were not found in the pharmacologically treated ultrasound-exposed group ( ${ }^{*} \mathrm{p}<0.05$ vs. control group, two-wayANOVA and Tukey test). Bars are Mean \pm SEM. US: ultrasound exposure.

\section{Effects of a 3-week ultrasound exposure on CBA mice}

In another validation experiment, male CBA mice were subjected to the same housing and experimental conditions as described in the main text. On the day after exposure, the social behaviours of the control and ultrasound-exposed mice were 
investigated as described elsewhere (Morozova et al., 2016; Pavlov et al., 2019). Serum corticosterone concentrations after 1week, 2 -week or 3 -week ultrasound exposures were studied as described above (see also Couch et al., 2013). Six or seven mice per group were used in this study.

Similarly to BALB/c mice, in comparison with naïve control groups, CBA mice exposed to a 3-week ultrasound showed decreased latency to attack, increased number of attacks and their duration in the social interaction test $(\mathrm{p}<0.05, t$-test, Fig. 4 A-C) and in the social the exploration test $(\mathrm{p}<0.05, t$-test, Fig.4 DF). In comparison with controls, a 3-week ultrasound exposure, but not 1-week or 2-week of ultrasound exposures resulted in a significant elevation of serum corticosterone concentrations $(\mathrm{p}<0.05$, one-way ANOVA and Tukey test, Fig.3G).

SOCIAL INTERACTION
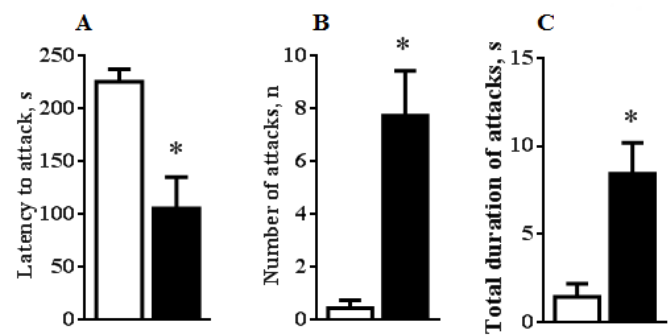

Control
US exposure

SOCIAL EXPLORATION

CORTICOSTERONE
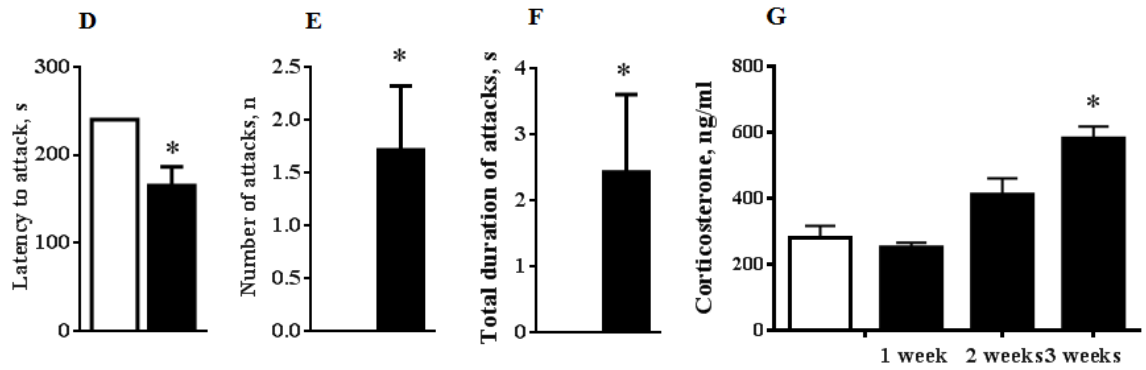
Supplemental figure 4. Ultrasound exposure induces aggressive behaviour and increases serum corticosterone concentrations in CBA mice. In the social interaction test, in comparison with naïve control groups, animals exposed to a 3 -week ultrasound showed (A) decreased latency to attack, (B) increased number of attacks and (C) increased duration of attacks. In the social exploration test, in comparison with controls, mice exposed to a 3-week ultrasound demonstrated (D) decreased latency to attack, (E) increased number of attacks and (F) increased duration of attacks $\left({ }^{*} \mathrm{p}<0.05\right.$ vs. control, $t$-test). (G) In comparison to controls, a 3-week ultrasound exposure, but not 1-week or 2-week ultrasound exposure resulted in a significant increase of serum corticosterone concentrations $\left({ }^{*} p<0.05\right.$ vs. control group, ANOVA and post-hoc Tukey test). Bars are means \pm SEMs; US - ultrasound exposure.

\section{Quantitative reverse transcription polymerase chain} reaction analysis (qRT-PCR)

Total mRNA was isolated from each sample with TRI Reagent (Invitrogen, Carlsbad, CA, USA). During first-strand cDNA synthesis 1 pg total RNA was converted into cDNA using random primers and Superscript III transcriptase (Invitrogen, Carlsbad, CA, USA). qRT-PCR was performed using the SYBR Green master mix (Bio-Rad Laboratories, Philadelphia, PA, USA) and the CFX96 Deep Well Real-Time PCR Detection System (BioRad, Hercules, CA, USA). qRT-PCR was performed in a $10 \mu \mathrm{l}$ reaction volume containing a SYBR Green master mix $(5 \mu \mathrm{l})$, RNase-free water $(3 \mu \mathrm{l})$, specific forward and reverse primers used at the concentration $20 \mathrm{pmol} / \mu \mathrm{l}(1 \mu \mathrm{l})$, cDNA $(1 \mu \mathrm{l})$.

Glyceraldehyde-3-phosphate dehydrogenase (GAPDH) was selected as a reference gene, since in previous experiments it was 
observed relatively low variability in its hippocampal expression in ultrasound model (Morozova et al., 2016). The initial denaturation step for qRT-PCR was at $95^{\circ} \mathrm{C}$ for $4 \mathrm{~min}$ followed by 40 cycles of denaturation at $95^{\circ} \mathrm{C}$ for 20 seconds. Annealing was at $54^{\circ} \mathrm{C}$ for 90 seconds.

Further details, as well as the sequences of primers used are listed in Supplementary Table 1 (see below); all primers were purchased from Evrogen, Moscow, Russia. All samples were run in triplicate. Data were normalized to GAPDH mRNA expression and calculated as relative-fold changes compared to control mice that were not exposed to ultrasound exposure, as described elsewhere (Morozova et al., 2016; Pavlov et al., 2017, 2019).

\section{Table 1. Sequences of primers for qRT-PCR}

\begin{tabular}{|c|c|c|}
\hline Gene & Forward primer 5'-3' & Reverse primer 5'-3' \\
\hline GAPDH & TGCACCACCAACTGCTTAG & GGATGCAGGGATGATGTTC \\
\hline GluA1 & GGACAACTCAAGCGTCCAGA & GTCGGTAGGAATAGCCCACG \\
\hline GluA2 & GCGTGGAAATAGAAAGGGCC & ACTCCAGTACCCAATCTTCCG \\
\hline GluA3 & ACCATCAGCATAGGTGGACTT & ACGTGGTAGTTCAAATGGAAGG \\
\hline GluA4 & GGCCAGGGAATTGACATGGA & CCTTTCGAGGTCCTGTGCTT \\
\hline $5-H T 6-R$ & GCATAGCTCAGGCCGTATGT & CACCACTGTGAGAGGTCCAC \\
\hline
\end{tabular}




\section{Western Blot assay}

Frozen tissue was treated with lysis buffer containing 20 $\mathrm{mM}$ of Tris- $\mathrm{HCl}(\mathrm{pH} 7.5), 450 \mathrm{mM}$ of $\mathrm{NaCl}, 1 \%$-solution of Triton $\mathrm{X}-100,1 \mathrm{mM}$ of EDTA, $1 \mathrm{mM}$ of $\mathrm{NaF}, 1 \mathrm{mM}$ of $\mathrm{Na}_{3} \mathrm{VO}_{4}$ and protease inhibitor (Roche Diagnostics, Indianapolis, IN, USA); 50 $\mu \mathrm{l}$ of buffer per $1 \mathrm{~g}$ of tissue was used. Samples were centrifuged at $16000 \mathrm{rpm}$ for $20 \mathrm{~min}$ at $4^{\circ} \mathrm{C}$, supernatant was collected and stored until use at $-20^{\circ} \mathrm{C} .25 \mu \mathrm{g}$ of protein from each sample was mixed with $35 \mathrm{\mu l}$ of Laemmli buffer. A sample of identical volume, comprising of $26 \mu \mathrm{l}$ of Laemmli buffer, $5 \mu \mathrm{l}$ of Page Ruler and $4 \mu \mathrm{l}$ of Magic Mark (Sigma, Munich, Germany) was used as a reference. For electrophoresis, samples were diluted in a solution containing MiliQ $\mathrm{H}_{2} \mathrm{O}, 1.5 \mathrm{M}$ of Tris Buffer (pH 8.8), 30\%-solution of Acrylamide, 10\%-solution of SDS Temed, 10\%-solution of ammonium persulfate (APS). For the next step, a solution containing MiliQ $\mathrm{H}_{2} \mathrm{O}, 0.5 \mathrm{M}$ of Tris Buffer (pH 8.8), 30\%-solution of Acrylamide, 10\%-solution of SDS Temed, 10\%-solution of APS and gel (Sigma, Munich, Germany) was used. The percentage of gel solution was adjusted to sizes of proteins of interest and was $20 \%$ for proteins of size of $4-40 \mathrm{kDa}, 12.5 \%$ for proteins of the size of $40-70 \mathrm{kDa}, 10 \%$ for proteins of the size of $70-100$, and $7.5 \%$ for proteins over $100 \mathrm{kDa}$. A buffer containing $25 \mathrm{mM}$ of Tris Base buffer, $192 \mathrm{mM}$ of Glycine (Sigma, Mannheim, Germany), 10\%solution of SDS and MiliQ $\mathrm{H}_{2} \mathrm{O}(\mathrm{pH}$ 8.3) was used for gel electrophoresis which was carried out under the constant voltages of $80 \mathrm{~V}$ and $130 \mathrm{~V}$. 
Polyvinylidene difluoride (PVDF) membrane $(9 \times 6 \mathrm{~cm}$, EMD Millipore, Billerica, MA, USA) was consequently incubated in a 99\%-methanol solution for 1 min (Brocacef, Amsterdam, the Netherlands), a MiliQ $\mathrm{H}_{2} \mathrm{O}$ for 5 min, and a transfer buffer for 15 min. The latter contained $25 \mathrm{mM}$ of Tris Base, $192 \mathrm{mM}$ of glycine, $20 \%$-solution of methanol, MiliQ $\mathrm{H}_{2} \mathrm{O}$ (pH 8.3). For the next step, blot "transfer sandwich" was composed of buffer soaked sponge, consisting of two buffer soaked Whatman filter papers, gel, activated membrane, ice-cold transfer buffer; the constant current of $300 \mathrm{~mA}$ was used for $2 \mathrm{~h} 30 \mathrm{~min}$.

Thereafter, the membrane was treated with a 5\%-dry milk solution the TBST, containing $50 \mathrm{mM}$ of Tris- $\mathrm{HCl}(\mathrm{pH}=8.2), 150$ $\mathrm{mM}$ of $\mathrm{NaCl}, 0.05 \%$-solution of Tween 20 (Sigma, Munich, Germany) for $1 \mathrm{~h}$ at the room temperature and subsequently incubated with primary antibodies (see Supplementary Table 2) at $4^{\circ} \mathrm{C}$ overnight that was followed by the incubation with respective horseradish peroxidase-conjugated secondary (HRP) antibodies (Sigma-Aldrich, St. Louis, MO, USA, for $2 \mathrm{~h}$ at room temperature on a roller. The membrane was washed in TBST three times, 5 min each time and placed on the plastic cover. Thereafter, Western Bright ${ }^{\mathrm{TM}}$ ECL kit (Advansta Inc, Menlo Park, CA, USA). A relative optical density of immunoreactive protein bands was examined using ImageJ software (NIH, Bethesda, MD, USA). Results were normalized to the relative intensity of the B-tubulin band that was selected as a reference protein as described elsewhere (Morozova et al., 2016; Pavlov et 
al., 2019). Blots were stripped by incubation with Restore Western Blot stripping Buffer (Thermo Scientific, Rockford, IL, USA) at the room temperature for $15 \mathrm{~min}$.

To normalize the data, the value of each protein of interest was expressed in percent from the concentration value of Btubulin, the reference protein. The choice of a reference protein was based on the previous observations where its expression was found to vary moderately across various experimental conditions as well as the linear representation of its signal intensity was demonstrated (Morozova et al., 2016; Pavlov et al., 2019).

\section{Determination of protein concentration}

Protein concentration was quantified using the BCA protein assay kit (Pierce, Rockford, IL, USA). The working reagent was prepared in accordance with manufacturer instructions. $25 \mu \mathrm{l}$ of each standard or sample preparations were pipetted into a microplate well, $200 \mu \mathrm{l}$ of the working reagent was added to each well and mixed thoroughly on a plate shaker for 30 seconds; assay was run in duplicates. The covered plate was incubated at $37^{\circ} \mathrm{C}$ for 30 minutes and cooled to room temperature for $10 \mathrm{~min}$. The absorbance was measured at $562 \mathrm{~nm}$ in a Biotek Microplate Reader (Biotek Instruments, Winooski, VT, USA). Ascent Software Program (Winooski, VT, USA) coupled to the microplate reader was used to calculate protein values based on comparing optical densities readings of the experimental samples with those obtained from the standard curve; the blank value was subtracted 
from all other optical densities readings. A standard curve was generated by plotting the average blank-corrected $562 \mathrm{~nm}$ measurements for each BSA standard vs. its concentration in $\mu \mathrm{g} / \mathrm{ml}$.

Table 2. Primary antibodies used in the Western blot assay

\begin{tabular}{|c|c|}
\hline Antibody & Dilution \\
\hline $\begin{array}{c}\text { Anti-6-tubulin (Abcam, Cambridge, MA, USA) } \\
\text { ab8227 }\end{array}$ & $1: 700$ \\
\hline $\begin{array}{c}\text { Anti-GluA2 (Abcam, Cambridge, MA, USA) } \\
\text { ab20673 }\end{array}$ & $1: 800$ \\
\hline $\begin{array}{c}\text { Anti-5-HT6-R (Abcam, Cambridge, MA, USA) } \\
\text { ab103016 } \\
\text { ab13552 }\end{array}$ & $1: 900$ \\
\hline Anti-PSD95 (Abcam, Cambridge, MA, USA) & $1: 700$ \\
\hline Anti-PSA-NCAM (Abcam, Cambridge, MA, USA) & $1: 800$ \\
\hline $\begin{array}{c}\text { ab5324-beta-catenin (Abcam, Cambridge, MA, USA) } \\
\text { ab32572 }\end{array}$ & $1: 1000$ \\
\hline
\end{tabular}

\section{Immunohistochemical analysis}

Immunohistochemical analyses were performed as described previously (Strekalova et al., 2002, 2015, 2018; Vignisse et al., 2016; Pavlov et al., 2019). To produce paraffin blocks of dissected hippocampi, PFA-fixed hemispheres were incubated 2 times for $1 \mathrm{~h}$ with $70 \%$-ethanol solution, for $1.5 \mathrm{~h}$ with $80 \%$ ethanol solution, for $1 \mathrm{~h}$ with $95 \%$-ethanol solution, 3 times for 1.5 h, in $100 \%$-ethanol solution, 3 times for 1.5 hour in xylene, for 2 $\mathrm{h}$, in paraffin first wax paraplast Xtra (at $58^{\circ} \mathrm{C}$ ), for $2 \mathrm{~h}$ paraffin second wax paraplast Xtra (at $58^{\circ} \mathrm{C}$ ) in cassettes (Sigma-Aldrich, 
St. Louis, MO, USA). Initially, a small amount of molten paraffin was dispensed in mold from paraffin reservoir. Tissue was transferred into the mold using warm forceps. Subsequently, the mold was placed on a cold plate for paraffin to solidify into a thin layer. Hot paraffin was added to the mold from the paraffin dispenser to cover the front surface of the plastic cassette. Then paraffin blocks were stored until use at the room temperature.

Samples were sectioned using a cryostat Leica CM 1850 cryostat (Leica Microsystems, Wetzlar, Germany). Serially cut sections were taken from lateral 3.6 to lateral $0.4 \mathrm{~mm}$ along the medial-lateral axis ahead of bregma (Paxinos and Franklin, 2001). Paraffin sections were picked up with forceps and placed on the surface of deionized water in the bath (Leica HI1210 Water bath, Wetzlar, Germany) at $40^{\circ} \mathrm{C}$. Subsequently, they were mounted onto glass slides. Sections were air dried for 30 minutes and then covered with coverslips.

To remove paraffin, sections were incubated 3 times for 5 min with xylene, 2 times for 10 min with $100 \%$-ethanol solution, 2 times for 10 min with 95\%-ethanol solution, 2 times for $10 \mathrm{~min}$ with $70 \%$-ethanol solution, 2 min for $10 \mathrm{~min}$ with 50\%-ethanol solution (Sigma-Aldrich, St. Louis, MO, USA) and 2 times for 5 min in ice-cold deionized water.

For antigen retrieval, 3 randomly selected hippocampal sections were incubated for 30 minutes at $90^{\circ} \mathrm{C}$ in $10 \mathrm{mM}$ sodium citrate and 0.05\% Tween 20, pH 6.0 (Applichem, Darmstadt, 
Germany) per each GluA assay. We used water bath for heatinduced epitope retrieval.

Blocking of non-specific staining was performed with $1 \%$ bovine serum albumin (BSA, Fluka, Buchs, Switzerland) in PBS, $\mathrm{pH} 7.4$, at room temperature for $1 \mathrm{~h}$ with all three assays. Sections were drained for a few seconds and wiped with a tissue paper. For surface staining of AMPARs, tissue was surface labelled with primary extracellular anti-GluA1/2/3 antibodies (ThermoFisher Scientific, Rockford, IL, USA, PA5-77415 / Alomone labs, Jerusalem, Israel, AGC-005-AG / Alomone labs, Jerusalem, Israel, AGC-010, respectively) overnight at $+4^{\circ} \mathrm{C}$, followed by Goat Anti-Mouse Alexa 568 secondary antibodies (ThermoFisher Scientific, Rockford, IL, USA, A-11004), Goat Anti-Mouse Alexa Fluor ${ }^{\circledR} 488$ (Abcam, Cambridge, MA, USA, ab150113), Goat antiMouse Alexa Fluor 405 Secondary Antibody (ThermoFisher Scientific, Rockford, IL, USA, A-31553) or Goat anti-Mouse Alexa Fluor 660 (ThermoFisher Scientific, Rockford, IL, USA, A-21074). Dilution was 1:600 for all cases.

The specificity of GluA antibodies was established in previous experiments conducted in our laboratory. Control immunofluorescence was performed in cells that express one or both proteins and specific siRNA was used to knock-down each individual protein and a loss in fluorescent staining was observed. For staining of internalized AMPARs, slices were then additionally fixed for 10 min with 4\% PFA and 4\% sucrose for 10 min and permeabilized with $0.2 \%$ Triton X-100 (Fluka, Buchs, 
Switzerland) for 1 min and then with $100 \%$ methanol (Fluka, Buchs, Switzerland) for 1min, thereafter internalized AMPARs were stained as previously reported. To count number of puncta (GluA puncta) dendritic structure was outlined in ImageJ software (NIH, Bethesda, MD, USA) manually by an experimentator, blind to experimental conditions, and appropriate stroke size for dendritic outline was set to quantify GluA. In addition, to exclude the influence of cell size, quantification of GluA was measured as total number of puncta/the region of dendritic structure. Three randomly selected $30 \mu \mathrm{m}$ dendritic regions were used per neuron and values were averaged. Images were acquired on a Leica SP8 confocal microscope with a 63x objective (Leica Microsystems, Mannheim, Germany). Internalization index (I) was calculated by dividing the number $(\mathrm{N})$ of puncta corresponding to the internalized staining by the values corresponding to the total staining (internalized+surface) as described in the mathematical formula below:

$$
\mathbf{I}_{\text {internalization }}=\frac{\mathbf{N}_{\text {(internalized puncta) }}}{N_{\text {(surface puncta) }}+\mathbf{N}_{\text {(internalized puncta) }}}
$$

Numbers from ultrasound-exposed group were normalized to the control group. 


\section{Effect of ultrasound exposure on liquid intake}

ANOVA revealed no significant ultrasound $\mathrm{x}$ treatment interaction in liquid intake after 1 day of ultrasound $\left(\mathrm{F}_{2,30}=0.0462, \mathrm{p}=0.9549\right)$, as well as no significant ultrasound effect $\left(\mathrm{F}_{1,30}=1.01, \mathrm{p}=0.3228\right)$ and no significant treatment effect $\left(\mathrm{F}_{2,30}=0.1885, \mathrm{p}=0.8292\right.$, two-way ANOVA; Fig.5). ANOVA revealed no significant ultrasound $\mathrm{x}$ treatment interaction in liquid intake after 2 days of ultrasound $\left(\mathrm{F}_{2,30}=0.04563\right.$, $\mathrm{p}=0.09555)$, no ultrasound effect $\left(\mathrm{F}_{1,30}=0.3194, \mathrm{p}=0.5762\right)$ and no treatment effect $\left(\mathrm{F}_{2,30}=0.03422, \mathrm{p}=0.9664\right.$, two-way ANOVA; Fig.5). ANOVA also revealed no significant ultrasound $\mathrm{x}$ treatment interaction in liquid intake after 3 days of ultrasound $\left(\mathrm{F}_{2,30}=0.0462, \mathrm{p}=0.9549\right)$, as well as no significant ultrasound effect $\left(\mathrm{F}_{1,30}=1.01, \mathrm{p}=0.3228\right)$ and treatment effect $\left(\mathrm{F}_{2,30}=0.1885\right.$, $\mathrm{p}=0.8292$, two-way ANOVA; Fig.5).

There was no significant ultrasound $\mathrm{x}$ treatment interaction in liquid intake during week $1\left(\mathrm{~F}_{2,33}=0.04708, \mathrm{p}=0.9541\right)$, week 2 $\left(\mathrm{F}_{2,33}=0.09412, \mathrm{p}=0.9104\right)$ and week $3\left(\mathrm{~F}_{2,33}=0.2271, \mathrm{p}=0.7981\right.$, two-way ANOVA; Fig.5). ANOVA revealed no ultrasound effect in liquid intake during week $1\left(\mathrm{~F}_{1,33}=1.34, \mathrm{p}=0.2553\right)$, week 2 $\left(\mathrm{F}_{1,33}=0.0003651, \mathrm{p}=0.9849\right)$ and week $3\left(\mathrm{~F}_{1,33}=1.464, \mathrm{p}=0.2349\right.$, two-way ANOVA; Fig.5). There was also no significant treatment effect liquid intake during week $1\left(\mathrm{~F}_{2,33}=0.3453\right.$, $\left.\mathrm{p}=0.7106\right)$, week $2\left(\mathrm{~F}_{2,33}=0.4436, \mathrm{p}=0.6455\right)$ and week $3\left(\mathrm{~F}_{2,33}=0.1722, \mathrm{p}=0.8426\right.$, two-way ANOVA; Fig.5). 

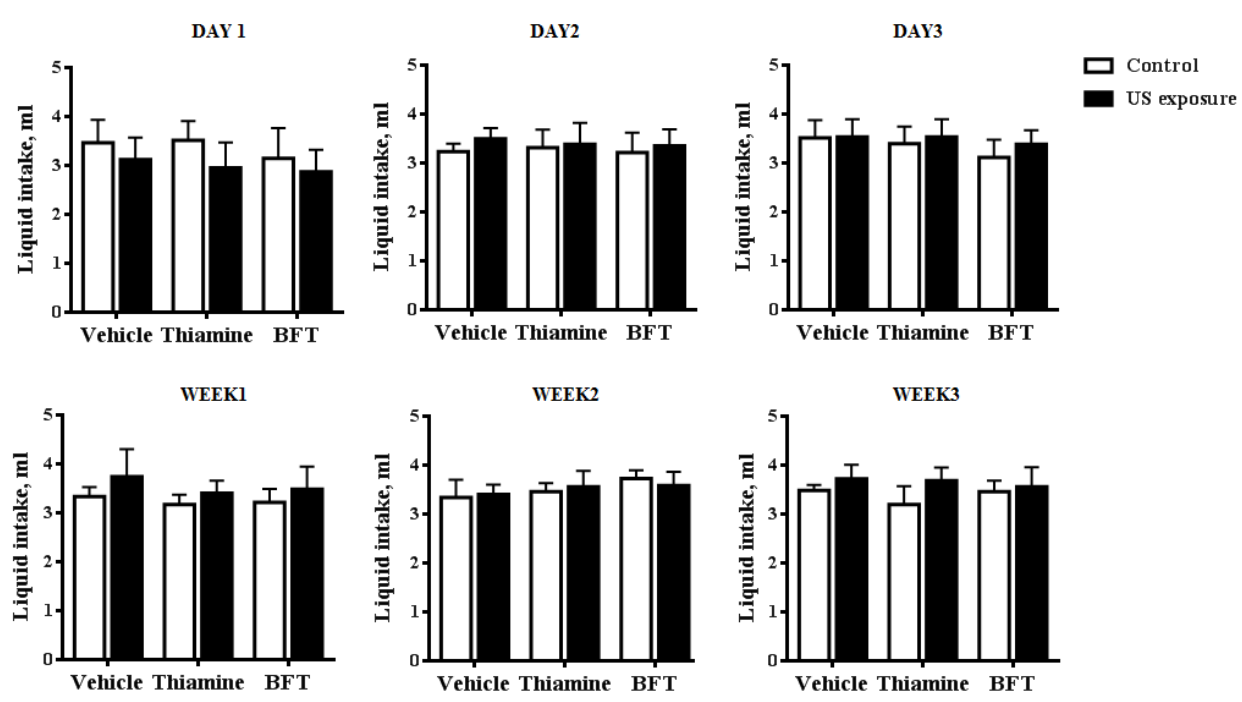

Supplemental figure 5. Drinking behavior during 3-week ultrasound exposure. 1, 2 or 3 days or 1, 2 or 3 weeks of exposure to the ultrasound or / and treatment with thiamine or benfotiamine did not result in any significant differences in average liquid intake ( $>0.05$ vs. control, two-way ANOVA and post hoc Tukey's test). Bars are Mean \pm SEM; US - ultrasound exposure.

\section{Measures of aggressive behavior in social interaction} test

ANOVA revealed a significant ultrasound $\mathrm{x}$ treatment interaction in latency to crawl-over $\left(\mathrm{F}_{2,37}=8.156, \mathrm{p}=0.0012\right)$ and total number of crawl-over behavior $\left(\mathrm{F}_{2,37}=8.377, \mathrm{p}=0.001\right)$. ANOVA revealed a significant ultrasound effect in latency to crawl-over $\left(\mathrm{F}_{1,37}=4.132, \mathrm{p}=0.0497\right)$ and total number of crawl-over behavior $\left(\mathrm{F}_{1,37}=5.615, \mathrm{p}=0.0231\right.$, two-way ANOVA; Fig.6A). ANOVA revealed a significant treatment effect in latency to 
crawl-over $\left(\mathrm{F}_{2,37}=7.614, \mathrm{p}=0.0018\right)$, total number of crawl-over behavior $\left(\mathrm{F}_{2,37}=9.198, \mathrm{p}=0.0006\right.$, two-way ANOVA; Fig.6A).

Post hoc analysis revealed significant decrease of latency to crawl-over in ultrasound-exposed non-treated group in comparison with naïve mice ( $\mathrm{p}=0.0003$, Tukey's test) and a significant increase of this parameter in ultrasound-exposed group treated with thiamine $(\mathrm{p}=0.0005$, Tukey's test $)$ or benfotiamine ( $p=0.0003$, Tukey's test; Fig.6A) in comparison with ultrasound-exposed non-treated group. Post hoc analysis also revealed significant increase of number of crawl-over behavior in ultrasound-exposed non-treated group in comparison with naïve mice ( $\mathrm{p}=0.0020$, Tukey's test) and a significant decrease of this parameter in ultrasound-exposed group treated with thiamine ( $p=0.0020$, Tukey's test), or benfotiamine ( $\mathrm{p}=0.0013$, Tukey's test; Fig.6A) in comparison with ultrasound-exposed non-treated group.

ANOVA revealed a significant ultrasound $\mathrm{x}$ treatment interaction in total number of followings $\left(\mathrm{F}_{2,37}=3.732, \mathrm{p}=0.0334\right)$ and no significant ultrasound $\mathrm{x}$ treatment interaction in latency to follow $\left(\mathrm{F}_{2,37}=0.1853, \mathrm{p}=0.8316\right)$ and total duration of following $\left(\mathrm{F}_{2,37}=0.5216, \mathrm{p}=0.5979\right.$; two-way ANOVA, Fig.6B). Also ANOVA revealed a significant ultrasound effect in total number of followings $\left(\mathrm{F}_{1,37}=4.189, \mathrm{p}=0.0479\right)$ and no significant ultrasound effect interaction in latency to follow $\left(\mathrm{F}_{1,37}=0.1467, \mathrm{p}=0.7039\right)$ and total duration of following $\left(\mathrm{F}_{1,37}=1.554, \mathrm{p}=0.2203\right.$; two-way ANOVA, Fig.6B). Furthermore, ANOVA revealed a significant 
treatment effect in total number of followings $\left(\mathrm{F}_{2,37}=3.871\right.$, $\mathrm{p}=0.0298$ ) and no significant treatment effect in latency to follow $\left(\mathrm{F}_{2,37}=1.304, \quad \mathrm{p}=0.2835\right)$ and total duration of following $\left(\mathrm{F}_{2,37}=1.412, \mathrm{p}=0.2565\right.$; two-way ANOVA, Fig.6B).

Post hoc analysis revealed significant increase of number of following behavior in ultrasound-exposed non-treated group in comparison with naïve mice ( $\mathrm{p}=0.0192$, Tukey's test) and a significant decrease of this parameter in ultrasound-exposed group treated with benfotiamine ( $\mathrm{p}=0.0103$, Tukey's test), but not thiamine ( $\mathrm{p}=0.3882$, Tukey's test; Fig.6B) in comparison with ultrasound-exposed non-treated group.

ANOVA revealed no significant ultrasound $\mathrm{x}$ treatment interaction in latency to tail rattling $\left(\mathrm{F}_{2,37}=1.134, \mathrm{p}=0.334\right)$, no significant ultrasound $\mathrm{x}$ treatment interaction in number of tail rattling $\left(\mathrm{F}_{2,37}=0.7683, \mathrm{p}=0.4719\right)$ and total duration of tail rattling $\left(\mathrm{F}_{2,37}=0.2059, \mathrm{p}=0.8150\right.$; two-way ANOVA, Fig.6C). Also ANOVA revealed no significant ultrasound effect in latency to tail rattling $\left(\mathrm{F}_{1,37}=1.085, \mathrm{p}=0.3052\right)$, no significant ultrasound effect in number of tail rattling $\left(\mathrm{F}_{1,37}=0.7349, \mathrm{p}=0.3975\right)$ and total duration of tail rattling $\left(\mathrm{F}_{1,37}=0.1969, \mathrm{p}=0.6601\right.$, two-way ANOVA, Fig.6C). Furthermore, ANOVA revealed no significant treatment effect in latency to tail rattling $\left(\mathrm{F}_{2,37}=3.252, \mathrm{p}=0.0514\right)$, no significant treatment effect in number of tail rattling $\left(\mathrm{F}_{2,37}=2.134\right.$, $\left.\mathrm{p}=0.1344\right)$ and total duration of tail rattling $\left(\mathrm{F}_{2,37}=1.853, \mathrm{p}=0.1727\right.$; two-way ANOVA, Fig.6C). 

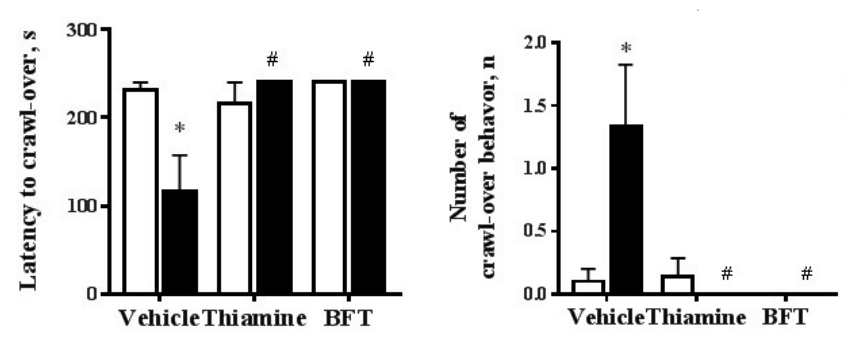

B

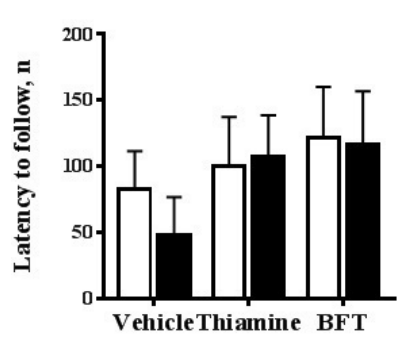

FOLLOWING BEHAVIOR

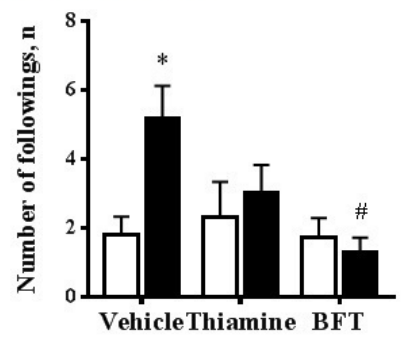

TAIL RATTLING
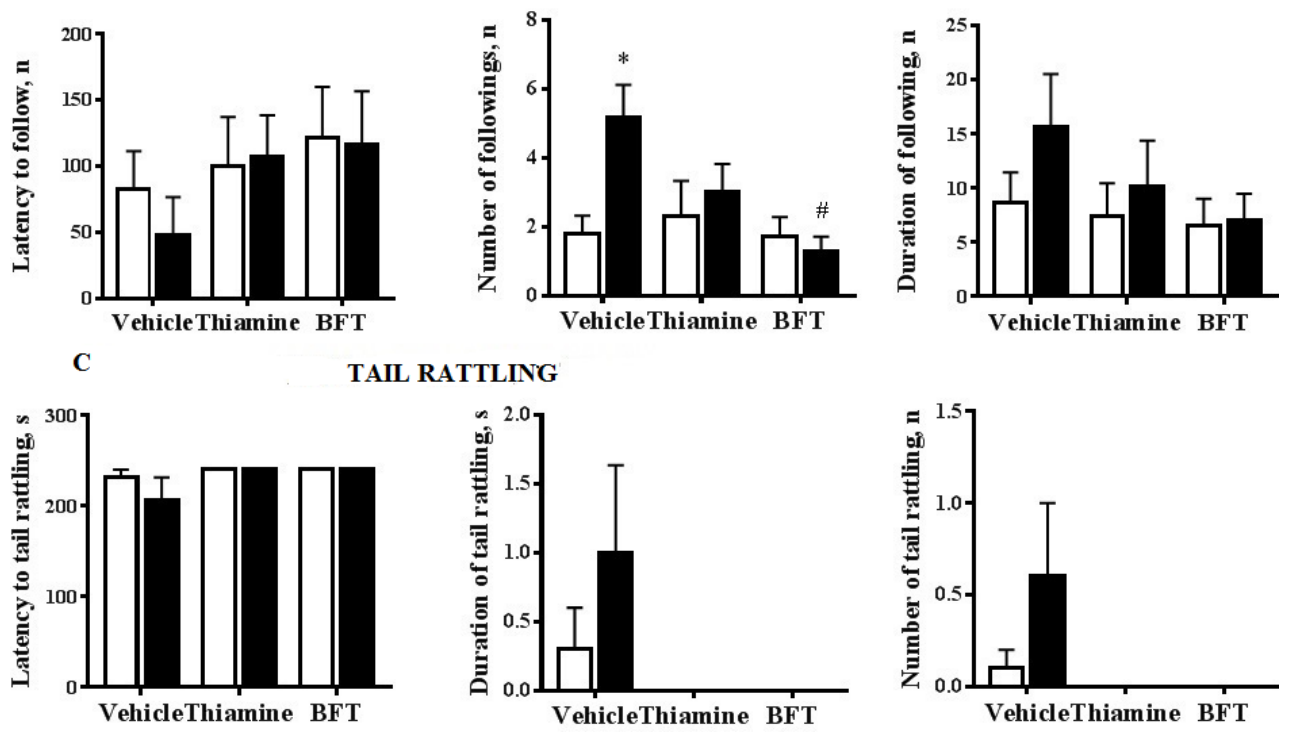

Supplemental Figure 6. Measures of aggressive-like behavior in the social interaction test. (A) Ultrasound-exposed vehicle-treated mice showed a significant decrease in latency to crawlover, total number and total duration of crawl-over behavior $\left({ }^{*} \mathrm{p}<0.05\right.$ vs. control, two-way ANOVA and post hoc Tukey's test). Both ultrasound-exposed thiamine-treated and benfotiamine-treated animals exhibited no change from control mice in these parameters and displayed significant differences from the ultrasound-exposed vehicletreated group $(\# p<0.05$ vs. ultrasound-exposed group, two-way ANOVA and post hoc Tukey's test). (B) Ultrasound-exposed vehicle-treated mice showed a significant increase in number of followings $\left({ }^{*} \mathrm{p}<0.05\right.$ vs. control, two-way ANOVA and post hoc Tukey's test). Ultrasoundexposed benfotiamine-treated, but not ultrasound-exposed thiamine- 
treated animals exhibited no change from control mice in these parameters and displayed significant differences from the ultrasoundexposed vehicle-treated group (\#p<0.05 vs. ultrasound-exposed group, two-way ANOVA and post hoc Tukey's test). There were no significant changes between groups in latency to interact and total duration of followings ( $p>0.05$ vs. ultrasound-exposed group, two-way ANOVA and post hoc Tukey's test). (C) There were no significant changes between groups in latency to tail rattling, number of tail rattling and total duration of tail rattling ( $>0.05$ vs. ultrasound-exposed group, two-way ANOVA and post hoc Tukey's test). Bars are Mean \pm SEM; US: ultrasound exposure.

\section{Measures of social behavior in the social interaction test}

In the social interaction test ANOVA revealed a significant ultrasound treatment interaction in total number of social contacts $\left(\mathrm{F}_{2,37}=5.849, \mathrm{p}=0.0062\right)$ a trend for ultrasound $\mathrm{x}$ treatment interaction in total duration of social contacts $\left(\mathrm{F}_{2,37}=3.233, \mathrm{p}=0.0505\right)$ and no significant ultrasound $\mathrm{x}$ treatment interaction in latency to interact $\left(\mathrm{F}_{2,37}=0.2399, \mathrm{p}=0.7879\right.$; two-way ANOVA, Fig.7). ANOVA revealed a significant ultrasound effect in total number of social contacts $\left(\mathrm{F}_{1,37}=4.441, \mathrm{p}=0.0419\right)$ and total duration of social contacts $\left(\mathrm{F}_{1,37}=8.78, \mathrm{p}=0.0052\right.$, two-way ANOVA), but not in latency to interact $\left(\mathrm{F}_{1,37}=0.06264, \mathrm{p}=0.08038\right.$ two-way ANOVA, Fig.7). Furthermore, ANOVA revealed significant treatment effect in total number of social contacts $\left(\mathrm{F}_{2,37}=3.619, \mathrm{p}=0.0367\right)$ and total duration of social contacts $\left(\mathrm{F}_{2,37}=3.856, \mathrm{p}=0.0299\right.$, two-way ANOVA), but not in latency to interact $\left(\mathrm{F}_{2,37}=0.02303, \mathrm{p}=0.9772\right.$ two-way ANOVA, Fig.7). Posthoc analysis revealed a significant decrease in the duration of social contacts in the ultrasound-exposed non-treated group of 
mice in comparison with naïve mice $(p=0.0069$, Tukey's test) and a reversal of this parameter in the ultrasound-exposed group that were treated with benfotiamine ( $\mathrm{p}=0.0173$, Tukey's test), but not thiamine ( $p=0.1563$, Tukey's test; Fig.7) in comparison with ultrasound-exposed non-treated group. Post-hoc analysis also revealed significant decrease of number of social contacts in ultrasound-exposed non-treated group in comparison with naïve mice ( $p=0.0045$, Tukey's test) and a significant increase of this parameter in ultrasound-exposed group treated with benfotiamine $(p=0.0047$, Tukey's test $)$, but not thiamine $(p=0.0853$, Tukey's test; Fig.7) in comparison with ultrasoundexposed non-treated group.
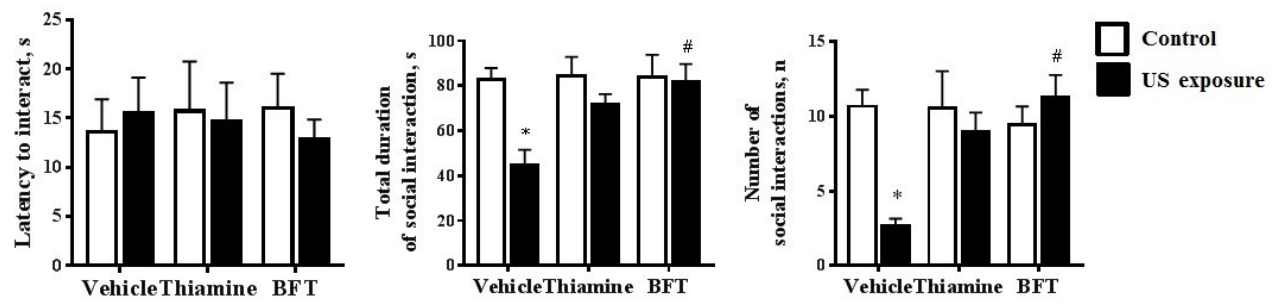

Supplemental Figure 7. Measures of social behavior in the social interaction test. Ultrasound-exposed vehicle-treated mice showed a significant decrease in the number and total duration of social contacts in comparison to controls $\left({ }^{*} \mathrm{p}<0.05\right.$ vs. control, two-way ANOVA and post hoc Tukey's test). Ultrasound-exposed benfotiaminetreated, but not thiamine-treated animals exhibited no change from control mice in these parameters and displayed significant differences from the ultrasound-exposed vehicle-treated group $(\# p<0.05$ vs. ultrasound-exposed group, two-way ANOVA and post hoc Tukey's test). There were no significant changes between groups in latency to interact ( $p>0.05$ vs. ultrasound-exposed group, two-way ANOVA and post hoc Tukey's test). Bars are Mean \pm SEM; US - ultrasound exposure. 
Protein carbonyl content in the brain of mice subjected to acute $24 \mathrm{~h}$-ultrasound exposure

ANOVA revealed no significant ultrasound $\mathrm{x}$ treatment interaction in protein carbonyl contents in the prefrontal cortex $\left(\mathrm{F}_{2,24}=0.2285, \mathrm{p}=0.7974\right)$ and the hippocampus $\left(\mathrm{F}_{2,24}=0.08463\right.$, $\mathrm{p}=0.9191)$. ANOVA revealed no significant ultrasound effect in protein carbonyl contents in the prefrontal cortex $\left(\mathrm{F}_{2,24}=0.01138\right.$, $\mathrm{p}=0.9159)$ and the hippocampus $\left(\mathrm{F}_{2,24}=0.382, \mathrm{p}=0.5424\right.$, two-way ANOVA; Fig.8). ANOVA revealed no significant treatment effect in protein carbonyl contents in the prefrontal cortex $\left(\mathrm{F}_{2,24}=0.2215\right.$, $\mathrm{p}=0.8029)$ and the hippocampus $\left(\mathrm{F}_{2,24}=0.9452, \mathrm{p}=0.4026\right.$, two-way ANOVA; Fig.8).

PREFRONTAL CORTEX

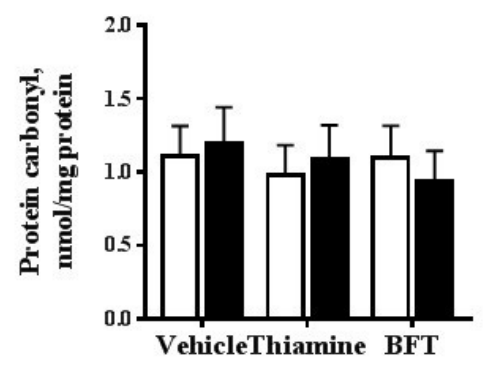

HIPPOCAMPUS

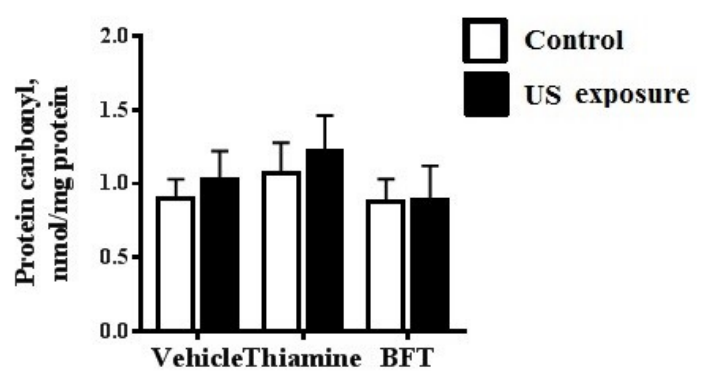

Supplemental Figure 8. Protein carbonyl content in the brain of mice subjected to acute $24 \mathrm{~h}$-ultrasound exposure. A 24$\mathrm{h}$ exposure of mice to the ultrasound exposure or / and treatment with thiamine or benfotiamine did not result in any significant differences in protein carbonyl contents as compared to control in prefrontal cortex and hippocampus ( $p>0.05$ vs.control, two-way ANOVA and post hoc Tukey's test). Bars are Mean \pm SEM; US - ultrasound exposure. 
Expression of GluA2 protein in the brain of mice subjected to acute 24-h-ultrasound and to chronic ultrasound exposure

For statistical analysis of chronic study, see main article paper. In acute study ANOVA revealed no significant ultrasound $\mathrm{x}$ treatment interaction in GluA2 protein concentration in the prefrontal cortex $\left(\mathrm{F}_{2,24}=0.03365, \mathrm{p}=0.9670\right)$ and the hippocampus $\left(\mathrm{F}_{2,24}=0.01006, \quad \mathrm{p}=0.99\right)$. ANOVA revealed no significant ultrasound effect in GluA2 protein concentration in the prefrontal cortex $\quad\left(\mathrm{F}_{1,24}=0.1298, \quad \mathrm{p}=0.7218\right)$ and the hippocampus $\left(\mathrm{F}_{1,24}=0.4025, \mathrm{p}=0.8427\right.$; Fig.9B $)$. ANOVA revealed no significant treatment effect in GluA2 protein concentration in the prefrontal cortex $\quad\left(\mathrm{F}_{2,24}=0.06891, \mathrm{p}=0.9336\right)$ and the hippocampus $\left(\mathrm{F}_{2,24}=0.1912, \mathrm{p}=0.8272\right.$; Fig.9B $)$.

Expression of 5-HT6 receptor protein in the brain of mice subjected to acute 24-h ultrasound and to chronic ultrasound exposure

For statistical analysis of chronic study, see main article paper. In the acute study, ANOVA revealed no significant ultrasound $\mathrm{x}$ treatment interaction in 5-HT6-R protein concentrations in prefrontal cortex $\left(\mathrm{F}_{2,24}=0.03861, \mathrm{p}=0.9622\right)$ and hippocampus $\left(\mathrm{F}_{2,24}=0.2175, \mathrm{p}=0.9784\right)$. ANOVA revealed no significant ultrasound effect in the 5-HT6-R protein concentrations in prefrontal cortex $\left(\mathrm{F}_{1,24}=0.73, \mathrm{p}=0.492\right)$ and hippocampus $\quad\left(\mathrm{F}_{1,24}=0.2034, \quad \mathrm{p}=0.6561, \quad\right.$ two-way ANOVA; 
Fig.10C). ANOVA revealed no significant treatment effect in 5HT6- $\mathrm{R}$ protein concentrations in prefrontal cortex $\left(\mathrm{F}_{2,24}=0.4147\right.$, $\mathrm{p}=0.6652)$ and hippocampus $\left(\mathrm{F}_{2,24}=0.1832, \mathrm{p}=0.8338\right.$, two-way ANOVA; Fig.10C).

PREFRONTAL CORTEX

A CHRONIC STUDY

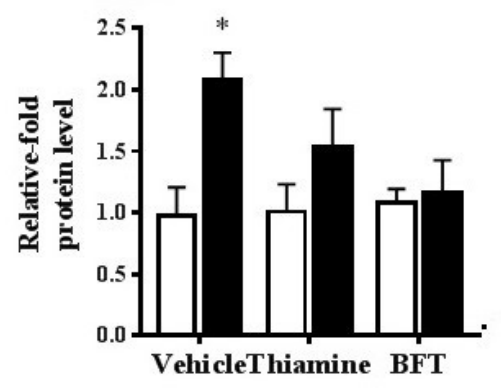

B ACUTE STUDY

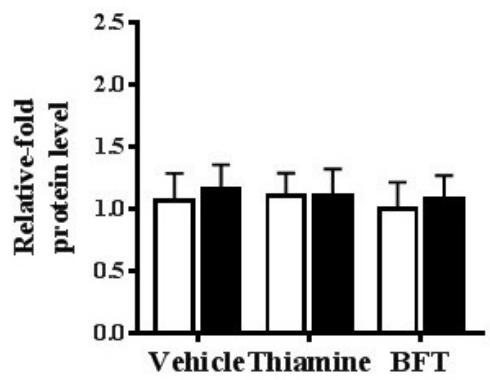

HIPPOCAMPUS

$\square$ Control

US exposure
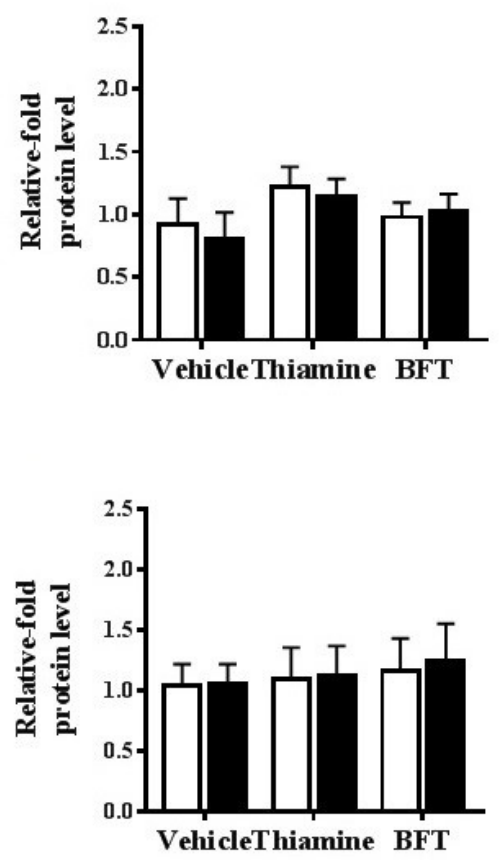

Supplemental Figure 9. Expression of GluA2 protein in the brain of mice subjected to acute $24 \mathrm{~h}$ and 3-weeks-ultrasound exposure. (A) In the chronic ultrasound experiment, ultrasoundexposed vehicle-treated mice demonstrated a significant increase of GluA2 relative fold protein levels in the prefrontal cortex $\left({ }^{*} \mathrm{p}<0.05\right.$ vs. control, two-way ANOVA and post hoc Tukey's test). There were no significant changes between groups in GluA2 relative fold protein levels in the hippocampus ( $>0.05$ vs. ultrasound-exposed group, two-way ANOVA and post hoc Tukey's test). (B) A 24-h exposure of mice to the ultrasound exposure or /and treatment with thiamine or benfotiamine 
did not result in any significant changes in GluA2 protein expression in the prefrontal cortex and hippocampus. Bars are Mean \pm SEM; US ultrasound exposure.

PREFRONTAL CORTEX

A CHRONIC STUDY

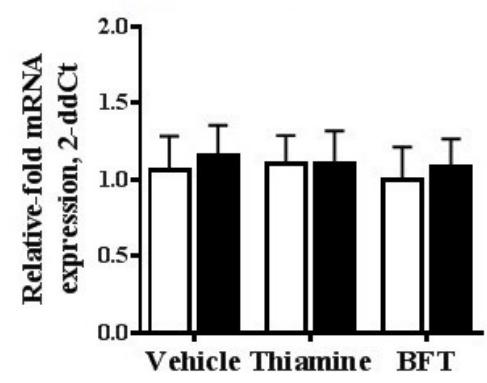

B

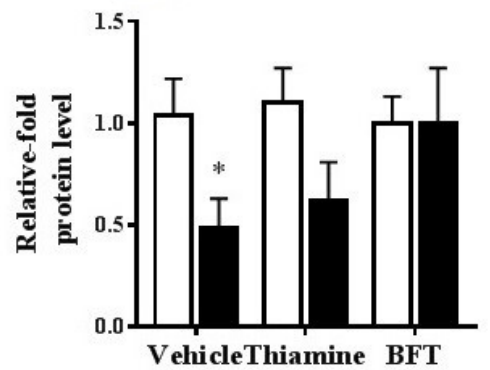

C ACUTE STUDY

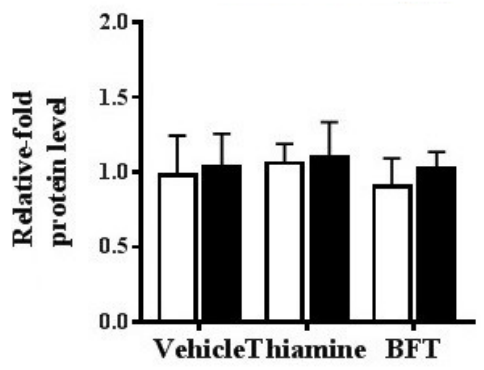

HIPPOCAMPUS

$\square$ Control US exposure
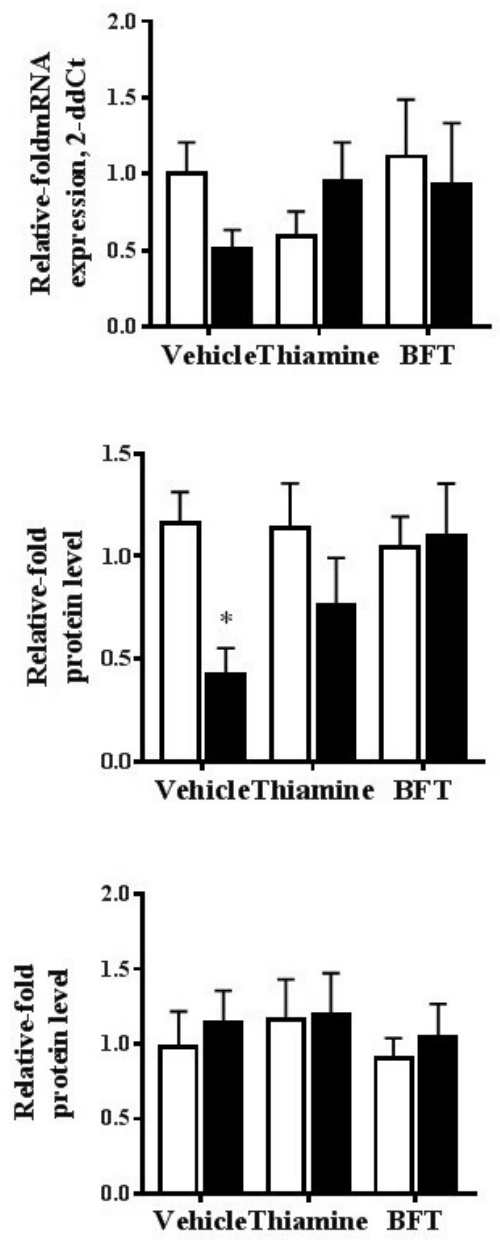

Supplemental Figure 10. Expression of 5-HT6 receptor in the brain of mice subjected to acute 24-h ultrasound and to chronic ultrasound exposure. (A) There were no significant changes between groups in 5-HT6 receptor mRNA concentrations in the prefrontal cortex ( $p>0.05$ vs. ultrasound-exposed group, two-way ANOVA and post hoc Tukey's test) and in hippocampus ( $>0.05$ vs. ultrasound-exposed group, two-way ANOVA and post hoc Tukey's test). 
(B) Ultrasound-exposed, vehicle-treated mice exhibited a significant decrease of 5 -HT6 receptor relative fold protein levels in prefrontal cortex (*p<0.05 vs. control, two-way ANOVA and post hoc Tukey's test) and hippocampus $\left({ }^{*} \mathrm{p}<0.05\right.$ vs. control, two-way ANOVA and post hoc Tukey's test). (C) A 24-h exposure of mice to the ultrasound exposure or /and treatment with thiamine or benfotiamine did not result in any significant changes in 5-HT6 receptor protein expression in the prefrontal cortex and the hippocampus. Bars are Mean \pm SEM; US ultrasound exposure.

\section{Effects of acute 24h-ultrasound exposure on brain} expression of plasticity factors

ANOVA revealed no significant ultrasound $\mathrm{x}$ treatment interaction in PSD95 protein concentration in the prefrontal cortex $\left(\mathrm{F}_{2,24}=0.08129, \quad \mathrm{p}=0.9222\right)$ and the hippocampus $\left(\mathrm{F}_{2,24}=0.01924, \quad \mathrm{p}=0.8262\right)$ and in PSA-NCAM protein concentrations in prefrontal cortex $\left(\mathrm{F}_{2,24}=0.1944, \mathrm{p}=0.8246\right)$ and hippocampus $\left(\mathrm{F}_{2,24}=1.242, \mathrm{p}=0.3067\right)$ as well as in B-catenin protein concentrations in prefrontal cortex $\left(\mathrm{F}_{2,24}=0.07846\right.$, $\mathrm{p}=0.9248)$ and hippocampus $\left(\mathrm{F}_{2,24}=0.2062, \mathrm{p}=0.8151\right)$. ANOVA revealed no significant ultrasound effect in PSD95 protein concentration in the prefrontal cortex $\left(\mathrm{F}_{1,24}=0.001114, \mathrm{p}=0.9737\right)$ and the hippocampus $\left(\mathrm{F}_{1,24}=0.001552, \mathrm{p}=0.9689\right)$ and in PSANCAM protein concentrations in prefrontal cortex $\left(\mathrm{F}_{1,24}=0.1392\right.$, $\mathrm{p}=0.7124)$ and hippocampus $\left(\mathrm{F}_{1,24}=0.3466, \mathrm{p}=0.5616\right)$ as well as in B-catenin protein concentrations in prefrontal cortex $\left(\mathrm{F}_{1,24}=0.1938, \quad \mathrm{p}=0.6637\right)$ and hippocampus $\left(\mathrm{F}_{1,24}=0.2946\right.$, $\mathrm{p}=0.8652$, two-way ANOVA; Fig.11). ANOVA revealed no significant treatment effect in PSD95 protein concentration in the 
prefrontal cortex $\left(\mathrm{F}_{2,24}=0.04788, \mathrm{p}=0.9533\right)$ and the hippocampus $\left(\mathrm{F}_{2,24}=0.1179, \quad \mathrm{p}=0.8893\right)$ and in PSA-NCAM protein concentrations in prefrontal cortex $\left(\mathrm{F}_{2,24}=0.1461, \mathrm{p}=0.8649\right)$ and hippocampus $\left(\mathrm{F}_{2,24}=0.3755, \mathrm{p}=0.6909\right)$ as well as in B-catenin protein concentrations in prefrontal cortex $\left(\mathrm{F}_{2,24}=0.1073\right.$, $\mathrm{p}=0.8987)$ and hippocampus $\left(\mathrm{F}_{2,24}=0.2979, \mathrm{p}=0.7451\right.$, two-way ANOVA; Fig.11).

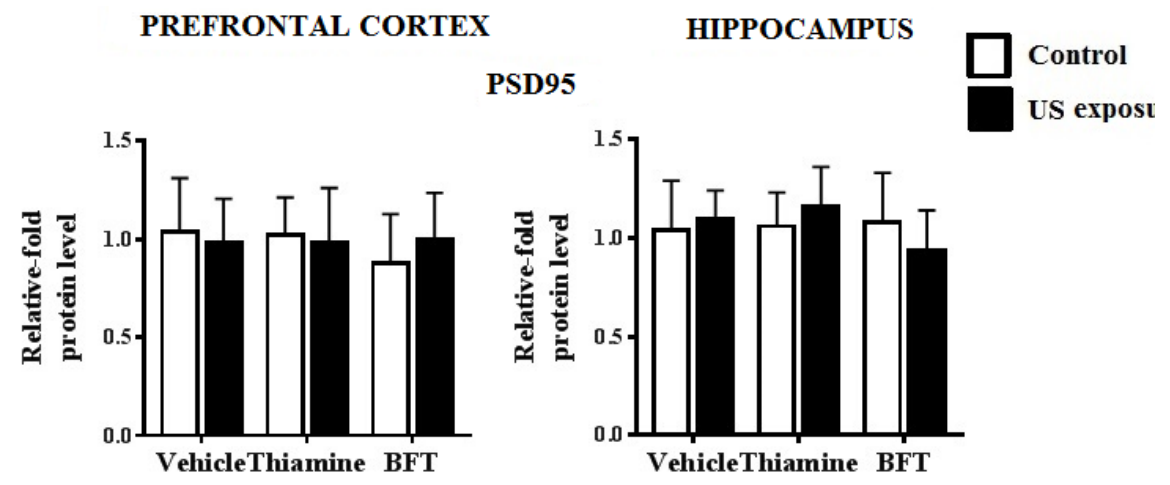

PSA-NCAM
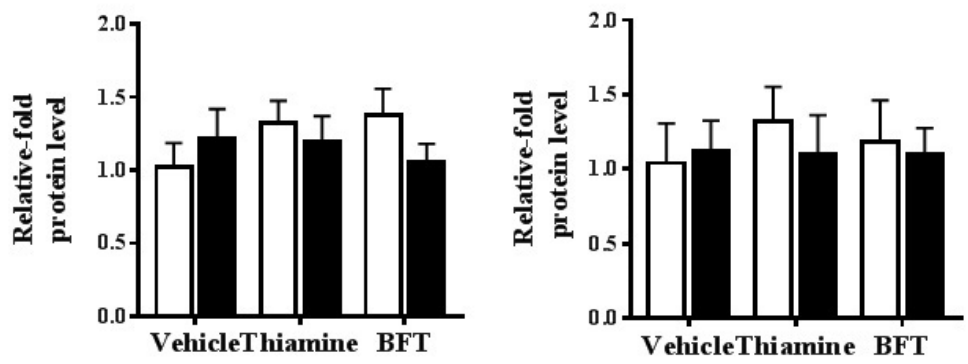

B-CATENIN
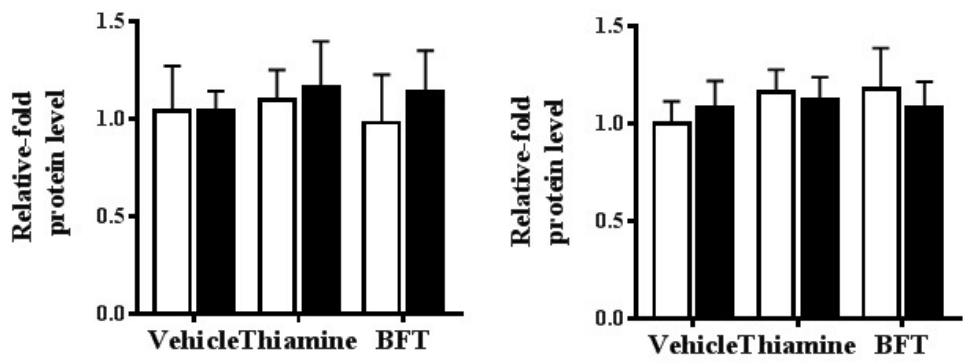
Supplemental Figure 11. Effects of acute $24 \mathrm{~h}$ ultrasound exposure on brain expression of plasticity factors. A 24-h exposure of mice to the ultrasound exposure or /and treatment with thiamine or benfotiamine did not result in any significant changes in PSD95 protein expression in the prefrontal cortex and hippocampus ( $p>0.05$ vs.control group, two-way ANOVA and post hoc Tukey's test). Mice subjected to acute exposure to ultrasound and /or thiamine or benfotiamine treatment also did not change PSA-NCAM receptor protein expression in the prefrontal cortex and the hippocampus, as well as and also B-catenin protein expression in the prefrontal cortex and the hippocampus hippocampus ( $p>0.05$ vs. control group, two-way ANOVA and post hoc Tukey's test). Thus, acute exposure to ultrasound and / or thiamine or benfotiamine treatment did not alter expression of measured neuroplasticity factors in the limbic brain structures. Bars are Mean \pm SEM; US - ultrasound exposure.

\section{Immunohistochemical analysis of surface protein} subunits of GluA

Immunohistochemical analysis revealed significant downregulation of surface GluA2 and GluA3 on a protein level (U=4, $\mathrm{p}=0.024$ and $\mathrm{U}=7, \mathrm{p}=0.019$, respectively) and a trend to decrease of GluA1 ( $\mathrm{U}=1, \mathrm{p}=0.062)$ in comparison with control, in groups exposed to a 3-week ultrasound (Fig.12, Mann-Whitney).

\section{Immunohistochemical analysis of internalized} protein subunits of GluA

Immunohistochemical analysis revealed significant upregulation of GluA1 and GluA2 on a protein level $(\mathrm{U}=5, \mathrm{p}=0.014$, $\mathrm{U}=6, \mathrm{p}=0.023$, respectively) and unchanged level of GluA3 (U=0, $\mathrm{p}=0.49$ ) in comparison with control, in groups exposed to a 3-week ultrasound (Fig.13, Mann-Whitney). 
GluA1

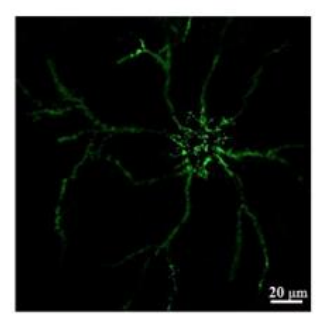

Control

US exposure
GluA2
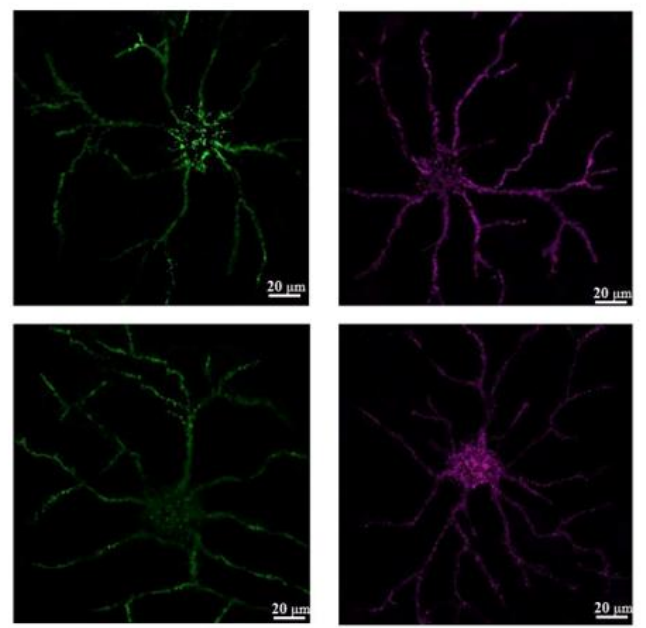

GluA3
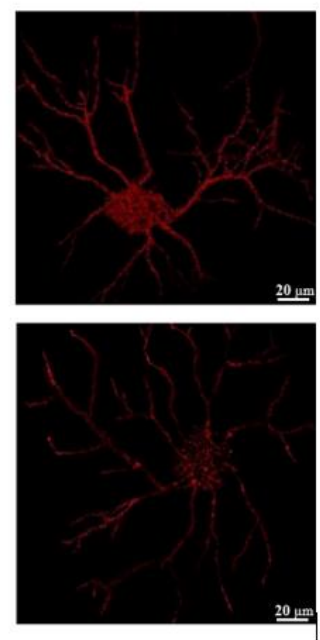
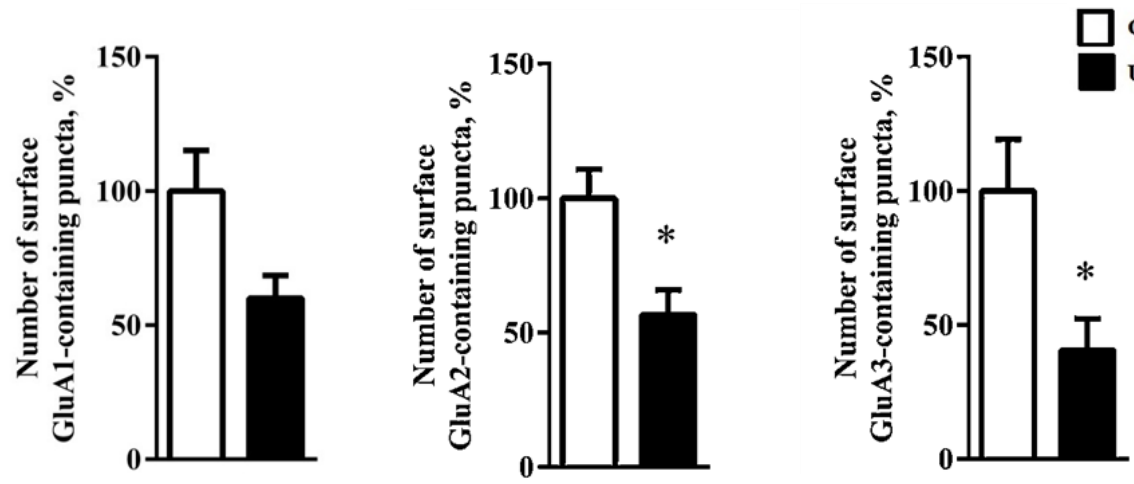

Supplemental Figure 12. Ultrasound-exposed, vehicle-treated mice exhibited a significant decrease of staining for GluA2 and GluA3, but not GluA1 receptor subunits in CA1 zone of hippocampus $\left({ }^{*} p<0.05\right.$ vs. control, Mann-Whitney test). Bars are Mean \pm SEM. 
GluA1

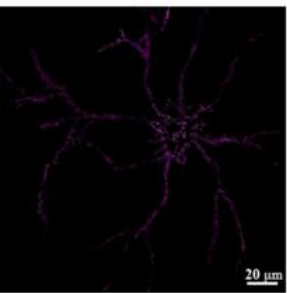

Control

US exposure

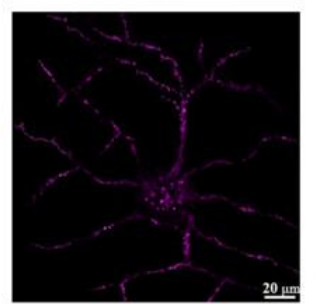

GluA2
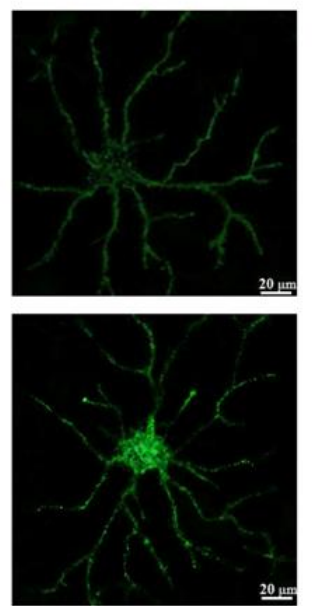

GluA3
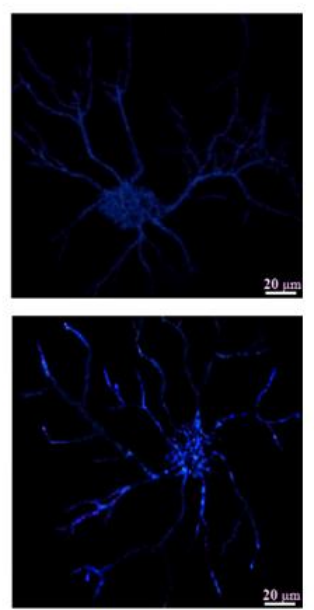

$\square$ Control

Us exposure
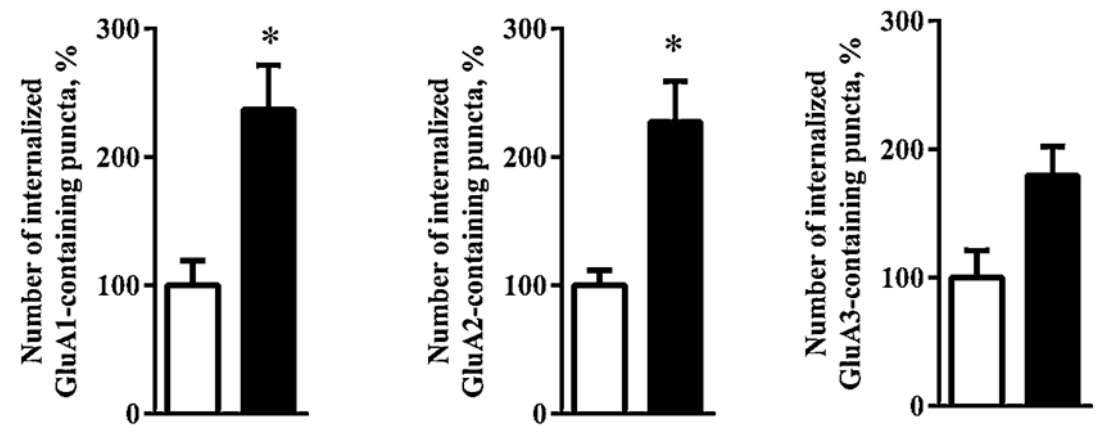

Supplemental Figure 13. Ultrasound-exposed, vehicle-treated mice exhibited a significant increase of GluA1 and GluA2, but not GluA3 receptor subunits in CA1 zone of hippocampus $\left({ }^{*} \mathrm{p}<0.05\right.$ vs.control, Mann-Whitney test). Bars are Mean \pm SEM. 


\section{Internalization index}

Internalization index was significantly elevated for all three GluA1, GluA2 and GluA3 subunits $(\mathrm{U}=3, \mathrm{p}=0.025, \mathrm{U}=2, \mathrm{p}=0.01$, $\mathrm{U}=5, \mathrm{p}=0.037$, respectively) in groups exposed to a 3 -week ultrasound exposure in comparison with control group (Fig.14).

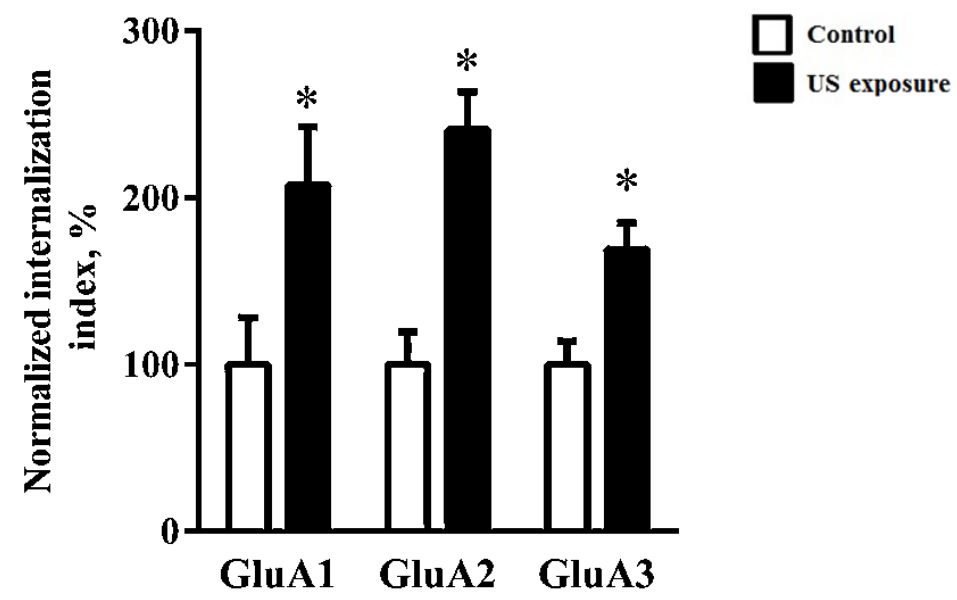

Supplemental Figure 14. Ultrasound exposure induces GluA internalization. Ultrasound-exposed, vehicle-treated mice exhibited a significant internalization of GluA1, GluA2 and GluA3 receptor subunits in CA1 zone of hippocampus $\left({ }^{*} \mathrm{p}<0.05\right.$ vs. control, Mann-Whitney test). All data were normalized to the corresponding control values. Bars are Mean \pm SEM; US - ultrasound exposure. 


\section{TABLES WITH STATISTICAL DATA}

Table 3. Summary of comparisons between mice that underwent ultrasound exposure and received thiamine or benfotiamine in social interaction, forced swim and novel cage tests. Two-way ANOVA and Tukey's post-hoc test were used. ANOVA revealed no significant ultrasound $\mathrm{x}$ treatment interaction in the latency to attack $\left(\mathrm{F}_{2,24}=0.0192, \mathrm{p}=0.989\right)$, total number of attacks $\left(\mathrm{F}_{2,24}=0.08517, \mathrm{p}=0.9186\right)$, total duration of attacks $\left(\mathrm{F}_{2,24}=0.96, \mathrm{p}=0.9087\right)$, latency to float $\left(\mathrm{F}_{2,24}=0.009438\right.$, $\mathrm{p}=0.9906)$, total duration of floating $\left(\mathrm{F}_{2,24}=0.1108, \mathrm{p}=0.8955\right)$, total number of rearings $\left(\mathrm{F}_{2,24}=0.3928, \mathrm{p}=0.678\right)$ and distance travelled $\left(\mathrm{F}_{2,24}=0.6966, \mathrm{p}=0.5081\right)$. ANOVA revealed no significant ultrasound effect in the latency to attack $\left(\mathrm{F}_{2,24}=0.2044\right.$, $\mathrm{p}=0.6552)$, total number of attacks $\left(\mathrm{F}_{2,24}=0.2621, \mathrm{p}=0.8727\right)$, total duration of attacks $\left(\mathrm{F}_{2,24}=0.005421, \mathrm{p}=0.9417\right)$, latency to float $\left(\mathrm{F}_{2,24}=0.5512, \mathrm{p}=0.8157\right)$, total duration of floating $\left(\mathrm{F}_{2,24}=1.597\right.$, $\mathrm{p}=0.2146)$, total number of rearings $\left(\mathrm{F}_{2,24}=0.004208, \mathrm{p}=0.9486\right)$ and distance travelled $\left(\mathrm{F}_{2,24}=0.03852, \mathrm{p}=0.846\right)$. ANOVA revealed no significant treatment effect in the latency to attack $\left(\mathrm{F}_{2,24}=0.07805, \mathrm{p}=0.9251\right)$, total number of attacks $\left(\mathrm{F}_{2,24}=0.1788\right.$, $\mathrm{p}=0.8374)$, total duration of attacks $\left(\mathrm{F}_{2,24}=0.2808, \mathrm{p}=0.7569\right)$, latency to float $\left(\mathrm{F}_{2,24}=0.1065, \mathrm{p}=0.8993\right)$, total duration of floating $\left(\mathrm{F}_{2,24}=0.09406, \mathrm{p}=0.9105\right)$, total number of rearings $\left(\mathrm{F}_{2,24}=0.9887\right.$, $\mathrm{p}=0.3819)$ and distance travelled $\left(\mathrm{F}_{2,24}=0.061, \mathrm{p}=0.941\right)$. 


\begin{tabular}{|c|c|c|c|c|c|c|}
\hline \multirow[b]{2}{*}{ Parameters } & \multicolumn{6}{|c|}{ Groups } \\
\hline & $\begin{array}{c}\text { Control } \\
\text { (C) }\end{array}$ & $\begin{array}{l}\text { Thiamine } \\
\text { (T) }\end{array}$ & $\begin{array}{l}\text { Benfotiamine } \\
\text { (B) }\end{array}$ & $\begin{array}{l}\text { Ultrasound- } \\
\text { exposed } \\
\text { (UE) }\end{array}$ & $\begin{array}{c}\text { Ultrasound- } \\
\text { exposed + } \\
\text { thiamine } \\
(\mathrm{UE}+\mathrm{T}) \\
\end{array}$ & $\begin{array}{c}\text { Ultrasound- } \\
\text { exposed + } \\
\text { Benfotiamine } \\
(\text { UE + B) }\end{array}$ \\
\hline $\begin{array}{l}\text { Latency to } \\
\text { attack, s }\end{array}$ & $154.4 \pm 33.97$ & $160.2 \pm 35.98$ & $146.2 \pm 25.61$ & $\begin{array}{c}148.8 \pm 27.72 \\
\text { vs. C: } \\
\text { p }>0.999\end{array}$ & $\begin{array}{c}140.6 . \pm 34.89 \\
\text { vs C: } \\
\text { p=0.998 } \\
\text { vs. UE: } \\
\text { p>0.999 }\end{array}$ & $\begin{array}{c}130.8 \pm 42.96 \\
\text { vs C: } \\
p=0.997 \\
\text { vs. UE: } \\
p=0.992\end{array}$ \\
\hline $\begin{array}{l}\text { Number of } \\
\text { attacks, } n\end{array}$ & $12 \pm 0.73$ & $1.4 \pm 0.68$ & $1.2 \pm 0.80$ & $\begin{array}{l}0.8 \pm 0.37 \\
\text { vs. C: } \\
p=0.996\end{array}$ & $\begin{array}{l}1,4 \pm 75 \\
\text { vs C: } \\
\text { p>0.999 } \\
\text { vs. UE: } \\
\text { p=0.992 }\end{array}$ & $\begin{array}{l}1,6 \pm 0.68 \\
\quad \text { vs C: } \\
\text { p>0.999 } \\
\text { vs. UE: } \\
p=0.991\end{array}$ \\
\hline $\begin{array}{c}\text { Total } \\
\text { duration of } \\
\text { attacks, s }\end{array}$ & $2.6 \pm 0.91$ & $4.2 \pm 0.47$ & $4 \pm 0.44$ & $\begin{array}{l}3.75 \pm 0.17 \\
\quad \text { vs. C: } \\
\text { p=0.998 }\end{array}$ & $\begin{array}{l}3 \pm 0.29 \\
\text { vs C: } \\
p=0.992 \\
\text { vs. UE: } \\
p=0.972\end{array}$ & $\begin{array}{l}3 \pm 0.50 \\
\text { vs C: } \\
p=0.998 \\
\text { vs. UE: } \\
p=0.997\end{array}$ \\
\hline $\begin{array}{l}\text { Latency to } \\
\text { float, } \mathrm{s}\end{array}$ & $67.8 \pm 13.26$ & $83.4 \pm 14.76$ & $73.4 \pm 11.84$ & $\begin{array}{c}94.8 \pm 12.90 \\
\text { vs. C: } \\
p=0.936\end{array}$ & $\begin{array}{c}84.3 \pm 14.43 \\
\text { vs C: } \\
\text { p=0.987 } \\
\text { vs. UE: } \\
p=0.999\end{array}$ & $\begin{array}{l}81 \pm 15.71 \\
\quad \text { vs C: } \\
\text { p>0.999 } \\
\text { vs. UE: } \\
p=0.963\end{array}$ \\
\hline $\begin{array}{c}\text { Total } \\
\text { duration of } \\
\text { floating, } s\end{array}$ & $94.6 \pm 13.15$ & $91 \pm 13.90$ & $108 \pm 6.34$ & $\begin{array}{c}90.6 \pm 16.79 \\
\text { vs. C: } \\
\text { p }>0.999\end{array}$ & $\begin{array}{c}85.2 \pm 19.43 \\
\text { vs C: } \\
\text { p=0.991 } \\
\text { vs. UE: } \\
\text { p>0.999 }\end{array}$ & $\begin{array}{c}96.2 \pm 16.37 \\
\text { vs C: } \\
\text { p>0.999 } \\
\text { vs. UE: } \\
\text { p>0.999 }\end{array}$ \\
\hline $\begin{array}{l}\text { Number of } \\
\text { rearings, } n\end{array}$ & $33.2 \pm 2.04$ & $36.6 \pm 2.26$ & $39.8 \pm 1.63$ & $\begin{array}{l}36.3 \pm 3.32 \\
\quad \text { vs. C: } \\
\text { p }>0.996\end{array}$ & $\begin{array}{l}39.3 \pm 2.48 \\
\quad \text { vs C: } \\
p=0.969 \\
\text { vs. UE: } \\
p>0.999\end{array}$ & $\begin{array}{l}39.5 \pm 6.67 \\
\quad \text { vs C: } \\
p=0.502 \\
\text { vs. UE: } \\
p=0.999\end{array}$ \\
\hline $\begin{array}{c}\text { Travelled } \\
\text { distance, } \mathrm{m}\end{array}$ & $2.04 \pm 0.22$ & $1.98 \pm 0.16$ & $2.22 \pm 0.37$ & $\begin{array}{l}2.26 \pm 0.17 \\
\quad \text { vs. C: } \\
p>0.988\end{array}$ & $\begin{array}{l}2.18 \pm 0.29 \\
\text { vs C: } \\
\text { p>0.992 } \\
\text { vs. UE: } \\
\text { p>0.999 }\end{array}$ & $\begin{array}{l}1.92 \pm 0.21 \\
\quad \text { vs C: } \\
p=0.954 \\
\text { vs. UE: } \\
p=0.925\end{array}$ \\
\hline
\end{tabular}


Table 4. Summary of comparisons between mice that underwent ultrasound exposure and received thiamine or benfotiamine in protein carbonyl contents. Two-way ANOVA and Tukey's post-hoc test were used (see ms text).

\begin{tabular}{|c|c|c|c|c|c|c|}
\hline \multirow{2}{*}{$\begin{array}{c}\text { Protein } \\
\text { carbonyl } \\
\text { content, } \\
\text { nmol/mg } \\
\text { protein }\end{array}$} & \multicolumn{6}{|c|}{ Groups } \\
\hline & $\begin{array}{c}\text { Control } \\
\text { (C) }\end{array}$ & $\begin{array}{c}\text { Thiamine } \\
\text { (T) }\end{array}$ & $\begin{array}{c}\text { Benfotiamine } \\
\text { (B) }\end{array}$ & $\begin{array}{l}\text { Ultrasound- } \\
\text { exposed (UE) }\end{array}$ & $\begin{array}{l}\text { Ultrasound- } \\
\text { exposed + } \\
\text { thiamine } \\
(\mathrm{UE}+\mathrm{T})\end{array}$ & $\begin{array}{c}\text { Ultrasound- } \\
\text { exposed }+ \\
\text { Benfotiamine } \\
(\text { UE + B) }\end{array}$ \\
\hline $\begin{array}{l}\text { Prefrontal } \\
\text { cortex }\end{array}$ & $1.1 \pm 0.21$ & $1 \pm 0.2$ & $1.1 \pm 0.22$ & $\begin{array}{c}1.2 \pm 0.28 \\
\text { vs. C: } p=0.999\end{array}$ & $\begin{array}{c}1.1 \pm 0.26 \\
\text { vs C: } p>0.969 \\
\text { vs. UE: } \\
\text { p=0.999 }\end{array}$ & $\begin{array}{c}1.0 \pm 0.21 \\
\text { vs C: } p>0.999 \\
\text { vs. UE: } \\
\text { p=0.996 }\end{array}$ \\
\hline Hippocampus & $0.9 \pm 0.13$ & $1.1 \pm 0.21$ & $0.9 \pm 0.15$ & $\begin{array}{c}0.8 \pm 0.11 \\
\text { vs. } \mathrm{C}: \mathrm{p}>0.999\end{array}$ & $\begin{array}{c}1.1 \pm 0.24 \\
\text { vs } \mathrm{C}: \mathrm{p}>0.999 \\
\text { vs. UE: } \\
\text { p }>0.999\end{array}$ & $\begin{array}{c}0.8 \pm 0.16 \\
\text { vs } C: p>0.999 \\
\text { vs. UE: } \\
\text { p }>0.999\end{array}$ \\
\hline
\end{tabular}


Table 5. Summary of comparisons between mice that underwent ultrasound exposure and received thiamine or benfotiamine in GluA2 and 5-HT6-R protein concentrations. Two-way ANOVA and Tukey's post-hoc test were used (see ms text).

\begin{tabular}{|c|c|c|c|c|c|c|}
\hline \multirow{2}{*}{ Parameters } & \multicolumn{6}{|c|}{ Groups } \\
\hline & $\begin{array}{l}\text { Control } \\
\text { (C) }\end{array}$ & $\begin{array}{c}\text { Thiamine } \\
\text { (T) }\end{array}$ & $\begin{array}{l}\text { Benfotiamine } \\
\text { (B) }\end{array}$ & $\begin{array}{l}\text { Ultrasound- } \\
\text { exposed (UE) }\end{array}$ & $\begin{array}{c}\text { Ultrasound- } \\
\text { exposed + } \\
\text { thiamine } \\
(\mathrm{UE}+\mathrm{T}) \\
\end{array}$ & $\begin{array}{c}\text { Ultrasound- } \\
\text { exposed }+ \\
\text { Benfotiamine } \\
\text { (UE + B) } \\
\end{array}$ \\
\hline $\begin{array}{l}\text { GluA2 } \\
\text { Prefrontal } \\
\text { cortex }\end{array}$ & $1.0 \pm 0.06$ & $1.1 \pm 0.14$ & $0.9 \pm 0.06$ & $\begin{array}{c}1.1 \pm 0.12 \\
\text { vs. } \mathbf{C}: p>0.999\end{array}$ & $\begin{array}{l}1.1 \pm 0.02 \\
\text { vs C: } \\
\text { p=0.966 } \\
\text { vs. UE: } \\
\text { p }=0.951\end{array}$ & $\begin{array}{c}1.1 \pm 0.03 \\
\text { vs C: } \\
\text { p }>0.9 \\
\text { UE ST: } \\
\text { p }=0.960\end{array}$ \\
\hline $\begin{array}{c}\text { GluA2 } \\
\text { Hippocampus }\end{array}$ & $1 \pm 0.09$ & $1.1 \pm 0.08$ & $1.1 \pm 0.16$ & $\begin{array}{c}1.2 \pm 0.13 \\
\text { vs. C: } p=0.960\end{array}$ & $\begin{array}{c}1 \pm 0.07 \\
\text { vs C: } \\
\text { p=0.988 } \\
\text { vs. UE: } \\
p>0.999\end{array}$ & $\begin{array}{l}1.1 \pm 0.11 \\
\text { vs C: } p=0.941 \\
\text { vs. UE: } \\
\text { p }>0.999\end{array}$ \\
\hline $\begin{array}{c}\text { 5-HT6-R } \\
\text { Prefrontal } \\
\text { cortex }\end{array}$ & $1.1 \pm 0.09$ & $1.1 \pm 0.08$ & $1.1 \pm 0.16$ & $\begin{array}{l}1.2 \pm 0.05 \\
\text { vs. C: } p=0.997\end{array}$ & $\begin{array}{l}1.1 \pm 0.05 \\
\text { vs C: } \\
p=0.971 \\
\text { vs. UE: } \\
p=0.999\end{array}$ & $\begin{array}{l}1.1 \pm 0.11 \\
\text { vs C: } \mathrm{p}=0.896 \\
\text { vs. UE: } \\
\mathrm{p}=0.999\end{array}$ \\
\hline $\begin{array}{c}\text { 5-HT6-R } \\
\text { Hippocampus }\end{array}$ & $1 \pm 0.24$ & $1.2 \pm 0.27$ & $0.9 \pm 0.14$ & $\begin{array}{c}1.1 \pm 0.23 \\
\text { vs. C: } p=0.996\end{array}$ & $\begin{array}{c}1 \pm 0.24 \\
\text { vs C: } \\
p=0.983 \\
\text { vs. UE: } \\
p>0.999\end{array}$ & $\begin{array}{l}1.2 \pm 0.19 \\
\text { vs C: } p>0.999 \\
\text { vs. UE: } \\
\text { p=0.996 }\end{array}$ \\
\hline
\end{tabular}


Table 6. Summary of comparisons between mice that underwent ultrasound exposure and received thiamine or benfotiamine in PSD95, PSA-NCAM and $\beta$-catenin protein concentrations. Two-way ANOVA and Tukey's post-hoc test were used (see ms text).

\begin{tabular}{|c|c|c|c|c|c|c|}
\hline \multirow{2}{*}{ Parameters } & \multicolumn{6}{|c|}{ Groups } \\
\hline & $\begin{array}{l}\text { Control } \\
\text { (C) }\end{array}$ & $\begin{array}{l}\text { Thiamine } \\
\text { (T) }\end{array}$ & $\begin{array}{l}\text { Benfotiamine } \\
\text { (B) }\end{array}$ & $\begin{array}{l}\text { Ultrasound- } \\
\text { exposed (UE) }\end{array}$ & $\begin{array}{c}\text { Ultrasound- } \\
\text { exposed + } \\
\text { thiamine } \\
(\mathrm{UE}+\mathrm{T})\end{array}$ & $\begin{array}{c}\text { Ultrasound- } \\
\text { exposed + } \\
\text { Benfotiamine } \\
\text { (UE + B) }\end{array}$ \\
\hline $\begin{array}{l}\text { PSD95 } \\
\text { Prefrontal } \\
\text { cortex }\end{array}$ & $1 \pm 0.27$ & $1 \pm 0.19$ & $0.9 \pm 0.25$ & $\begin{array}{c}1 \pm 0.26 \\
\text { vs. } \mathrm{C}: \mathrm{p}>0.999\end{array}$ & $\begin{array}{l}1 \pm 0.33 \\
\text { vs C: } \\
\text { p }>0.999 \\
\text { vs. UE: } \\
p>0.999\end{array}$ & $\begin{array}{c}1 \pm 0.24 \\
\text { vs } C: p>0.999 \\
\text { vs. UE: } \\
p>0.992\end{array}$ \\
\hline $\begin{array}{c}\text { PSD95 } \\
\text { Hippocampus }\end{array}$ & $1 \pm 0.25$ & $1.1 \pm 0.17$ & $1.1 \pm 0.25$ & $\begin{array}{c}1.1 \pm 0.16 \\
\text { vs. } \mathrm{C}: \mathrm{p}>0.999\end{array}$ & $\begin{array}{l}1,2 \pm 23 \\
\text { vs C: } \\
p=0.998 \\
\text { vs. UE: } \\
\text { p>0.999 }\end{array}$ & $\begin{array}{c}1 \pm 0.22 \\
\text { vs C: } p>0.999 \\
\text { vs. UE: } \\
p=0.994\end{array}$ \\
\hline $\begin{array}{l}\text { PSA-NCAM } \\
\text { Prefrontal } \\
\text { cortex }\end{array}$ & $1 \pm 0.27$ & $1.3 \pm 0.27$ & $1.2 \pm 0.28$ & $\begin{array}{c}1 \pm 0.17 \\
\text { vs. } \mathrm{C}: \mathrm{p}=0.999\end{array}$ & $\begin{array}{l}1.1 \pm 0.30 \\
\text { vs C: } \\
\text { p }>0.999 \\
\text { vs. UE: } \\
\text { p>0.999 }\end{array}$ & $\begin{array}{c}1.2 \pm 0.19 \\
\text { vs C: } p>0.999 \\
\text { vs. UE: } \\
\text { p }>0.999\end{array}$ \\
\hline $\begin{array}{l}\text { PSA-NCAM } \\
\text { Hippocampus }\end{array}$ & $1 \pm 0.17$ & $1.3 \pm 0.16$ & $1.4 \pm 0.18$ & $\begin{array}{c}1.1 \pm 0.18 \\
\text { vs. } \mathrm{C}: \mathrm{p}=0.956\end{array}$ & $\begin{array}{l}1.3 \pm 0.19 \\
\text { vs C: } \\
p=0.971 \\
\text { vs. UE: } \\
p>0.999\end{array}$ & $\begin{array}{c}1.1 \pm 0.13 \\
\text { vs } \mathrm{C}: \mathrm{p}>0.999 \\
\text { vs. UE: } \\
\mathrm{p}=0.990\end{array}$ \\
\hline $\begin{array}{l}\beta \text {-catenin } \\
\text { Prefrontal } \\
\text { cortex }\end{array}$ & $1 \pm 0.23$ & $1.1 \pm 0.15$ & $1 \pm 0.25$ & $\begin{array}{c}1 \pm 0.12 \\
\text { vs. } \mathrm{C}: \mathrm{p}>0.999\end{array}$ & $\begin{array}{l}1.2 \pm 0.12 \\
\text { vs C: } \\
p=0.998 \\
\text { vs. UE: } \\
p=0.998\end{array}$ & $\begin{array}{c}1.2 \pm 0.23 \\
\text { vs C: } p=0.999 \\
\text { vs. UE: } \\
\text { p }>0.999\end{array}$ \\
\hline $\begin{array}{c}\beta \text {-catenin } \\
\text { Hippocampus }\end{array}$ & $1.1 \pm 0.11$ & $1.2 \pm 0.12$ & $1.2 \pm 0.21$ & $\begin{array}{c}1.1 \pm 0.16 \\
\text { vs. } \mathrm{C}: \mathrm{p}>0.998\end{array}$ & $\begin{array}{c}1.2 \pm 0.13 \\
\text { vs C: } \\
\text { p=0.999 } \\
\text { vs. UE: } \\
\text { p>0.999 }\end{array}$ & $\begin{array}{c}1.1 \pm 0.15 \\
\text { vs C: } p=0.991 \\
\text { vs. UE: } \\
\text { p }>0.999\end{array}$ \\
\hline
\end{tabular}




\section{Chapter 3}

\section{Ultrasound stress compromises the}

correlates of emotional-like states and brain AMPAR expression in mice: effects of antioxidant and anti-inflammatory herbal treatment 
Chapter 3. Ultrasound stress compromises the correlates of emotional-like states and brain AMPAR expression in mice: effects of antioxidant and antiinflammatory herbal treatment

João Pedro Costa-Nunes ${ }^{1,2,3^{*}}$, Anna Gorlova ${ }^{2,3, *}$, Dmitrii Pavlov $^{2,3,4^{*}}$, Raymond Cespuglio 2,5 , Anna Gorovaya ${ }^{2}$, Andrei Proshin $^{6}$, Alexei Umriukhin ${ }^{2,6}$, Eugene Ponomarev ${ }^{7}$, Alan Kalueff ${ }^{8,9}$, Tatyana Strekalova ${ }^{2,3,4}$, Careen Schroeter $\$ 10$

${ }^{1}$ Instituto de Medicina Molecular João Lobo Antunes, Faculdade de Medicina da Universidade de Lisboa, Av. Professor Egas Moniz, 1649028 Lisboa, Portugal; 2I.M. Sechenov First Moscow State Medical University, Institute of Molecular Medicine, Laboratory of Psychiatric Neurobiology and Department of Normal Physiology, Trubetskaya Str. 8-2, 119991, Moscow, Russia; ${ }^{3}$ School for Mental Health and Neuroscience, Department of Psychiatry and Neuropsychology, Maastricht University, Universiteitssingel 40, 6229ER, Maastricht, The Netherlands; ${ }^{4}$ Institute of General Pathology and Pathophysiology, Baltiiskaya str, 8, Moscow 125315, Russia; ${ }^{5}$ Neuroscience Research Center of Lyon, C. Bernard University of Lyon, 95 Bd Pinel 69675 Bron, France; ${ }^{6}$ Research Institute of Normal Physiology, Baltiiskaya str, 8, Moscow, Russia, 125315, Moscow, Russia; ${ }^{7}$ School of Biomedical Sciences, Faculty of Medicine, The Chinese University of Hong Kong,

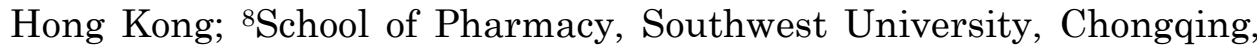
China; ${ }^{9}$ Institute of Translational Biomedicine, St.Petersburg State University, Universitetskaya nab. 7-9, St.-Petersburg 199034, Russia; ${ }^{10}$ Department of Preventive Medicine, Maastricht Medical Center Annadal, Becanusstraat 17, 6216BX Maastricht, The Netherlands.

*Authors contributed equally

Running title: Emotional stress and AMPAR in mice 
\#For correspondence:

Dr. Careen A. Schroeter

Department of Preventive Medicine

Maastricht Medical Center Annadal

Becanusstraat 17, 6216 BX Maastricht, The Netherlands

Tel: +31433875669

E-mail: careen.schroeter@web.de

\section{Abstract}

The modern lifestyle is associated with exposure to "psychological" or "emotional" stress. A growing portion of the population is exposed to emotional stress that results in a high incidence of anxiety disorders, a serious social problem. With this rise, there is a need for understanding the neurobiological causes of stress-induced anxiety and to offer safe remedies for this condition. Side effects of existing pharmaceuticals necessitate the search for alternatives. Having fewer adverse effects than classic remedies, natural extract-based therapies can be a promising solution. Here, we applied a model of emotional stress in BALB/c mice using ultrasound exposure to evoke the signs of anxiety-like behavior. We examined the behavioral and molecular impact of ultrasound and administration of herbal antioxidant/antiinflammatory treatment (HAT) on AMPA receptor expression, markers of plasticity, inflammation and oxidative stress. A 3- 
week ultrasound exposure increased scores of anxiety-like behaviors in the standard tests and altered hippocampal expression as well as internalization of AMPA receptor subunits GluA1-A3. Concomitant treatment with HAT has prevented increases of anxiety-like behaviors and other behavioral changes, normalized hippocampal malondialdehyde content, GSK3B and pro-inflammatory cytokines $\mathrm{Il}-18$ and Il-6, and the number of Ki67-positive cells. Levels of malondialdehyde, a common measure of oxidative stress, significantly correlated with the investigated end-points in stressed, but not in non-stressed animals. Our results emphasize the role of oxidative stress in neurobiological abnormalities associated with experimentally induced condition mimicking emotional stress in rodents and highlight the potential therapeutic use of anti-oxidants like herbal compositions for management of stress-related emotional disturbances within the community.

Keywords: ultrasound stress, anxiety-like behaviour, oxidative stress, AMPA glutamate receptors, pro-inflammatory cytokines, mice.

\section{Introduction}

The incidence of anxiety disorders is currently the highest of all mental disorders (Kessler et al., 2012; Bandelow and Michaelis, 2015). As for instance, 20\% of adults are diagnosed with generalized anxiety alone (Grenier et al., 2019). Anxiety disorders are frequently accompanied by increased aggression, 
violence, self-harm, and suicide (Stone et al., 2016). "Psychological" or "emotional" stress resulting from the adverse experience of non-material nature is the most common cause of these conditions (Fontes et al., 2014). At the same time, side effects of traditional anti-anxiety drugs are well-documented: benzodiazepines, the most widely used anxiolytics, cause rebound anxiety, insomnia and memory impairments (Uzun et al., 2010); barbiturates induce drowsiness and balance problems, disrupt learning and reproductive functions (Anderson et al., 2018), selective serotonin re-uptake inhibitors (SSRIs) evoke irritability and restlessness (Orsolini and Bellantuono, 2015), as well as fatigue, nausea and insomnia (Quagliato et al., 2019). That said, there is a need for an effective and safe pharmacological treatment, and herbal medicine appears as an attractive solution (Bystritsky et al., 2012) affordable for low-income patients (Oyebode et al., 2016). However, with an increase in selfmedication with herbal remedies, there is a need for better understanding of their mechanisms of action to prevent potential adverse effects (McIntyre et al., 2015).

Herbal medicine is known to normalize behavioral correlates of anxiety-like state and stress-associated changes experimentally induced in small rodents. This was shown in mice receiving chlorogenic acid extract from Prunus domestica (Bouayed et al, 2007) or Beta vulgaris during restraint stress (Sulakhiya et al., 2016) and in rats treated with an extract from Ulva spiralis (Violle et al., 2018). Herbal treatments with stress- 
reducing properties diminish oxidative stress, one of the causes of anxiety (Salim, 2017; Umukoro et al., 2018) and aggression (Schiavone et al., 2015).

Here, we investigated the potential anti-stress/anti-anxiety properties of a herbal antioxidant/anti-inflammatory treatment (HAT), a composition, containing seaweed, ginger, lemon, orange elderberries and other elements at the concentrations exploited in human and animal studies (Supplementary Table 1). A recently established rodent model of "emotional stress" was used, where mice and rats are exposed to the ultrasound of alternating frequencies $(25-45 \mathrm{kHz})$ that mimics rodent vocalizations emitted during conditions associated with "negative emotional state" (Morozova et al., 2016; Pavlov et al., 2019; Gorlova et al., 2019). A 3-week exposure of BALB/c mice to ultrasound stress increases behavioral correlates of aggressive behavior, anhedonia and helplessness. Ultrasound stress also increases levels of proinflammatory cytokines and Glycogene-Synthase-kinase-3-beta (GSK3B) in the brain, oxidative stress markers glutathione and malondialdehyde, decreases the levels of plasticity markers, and dysregulates gene expression of AMPA receptor subunits GluA1A3, but not GluA4 (Gorlova et al., 2019; Pavlov et al., 2019). Compounds with anti-oxidative properties prevent most of these changes (Gorlova et al, 2019).

In this study, we investigated the effect of ultrasound stress on the behavioral signs of anxiety-like behavior and internalization of GluA1-A3 AMPA receptor subunits in the 
hippocampus, one of the key brain structures involved in emotional regulation during stress (Campbell and MacQueen, 2004), in mice. We additionally investigated whether abovementioned new herbal antioxidant/anti-inflammatory treatment (HAT) can affect these measures induced by ultrasound stress, and alter hippocampal content of oxidative stress marker malondialdehyde (Ayala et al., 2014), as it was suggested by previous studies with applied here model (Gorlova et al., 2019; Pavlov et al., 2019). These changes were related with the abovelisted end-points and to hippocampal expression of GSK-3B, and Akt, two reciprocally related regulators of distress, plasticity and neuroinflammation (Beurel et al., 2015), as well as cytokines: interleukin (IL) IL-1B, IL-6, IL-15, tumor necrosis factor (TNF), and to the aggression scores.

\section{Materials and Methods}

\subsection{Animals}

$\mathrm{BALB} / \mathrm{c}$ male mice, 10-12-week-old, were obtained from certified Charles River provider and housed individually under standard laboratory conditions $\left(22 \pm 1^{\circ} \mathrm{C}, 55 \%\right.$ humidity, reversed 12-h light/dark cycle with lights on at 19:00, food and water ad libitum). Experimental procedures were set up in accordance with Directive 2010/63/EU and approved by the veterinarian committee of C.Bernard University. All efforts were undertaken to minimize the potential discomfort of the animals. 


\subsection{Study design}

Mice were weighed and assigned to one of six experimental groups: 1) control (non-stressed) non-treated mice, 2) control mice treated with HAT-solvent, 3) control HAT-treated mice, 4) stressed non-treated mice, 5) stressed mice treated with HATsolvent, 6) stressed HAT-treated mice, each comprising in 6-8 animals. Mice were subjected to a 3-week ultrasound of unpredictably alternating frequencies 24/7 (see below), while receiving respective treatments, body weight was measured. Twenty-four hours after, their behavior was subsequently investigated in the O-maze, open field and resident-intruder paradigms, with 2-h inter-test intervals. Applied experimental scheme was designed to reduce the potential stress of the behavioral testing. Mice were killed $16 \mathrm{~h}$ after the end of the last behavioral test. Brains and right and left hippocampi were dissected using previously established method (Pavlov et al., 2019; see below) for immunohistochemical study of AMPA receptor subunits GluA1-A3, neuronal marker NeuN and proliferation marker Ki67, or quantitative reverse transcriptase PCR (qRT-PCR) of IL-1B, Il-6, TNF, GSK3B, Akt and malondialdehyde (MDA) assays, respectively (Fig.1; see below). Above-described study design was adapted from similar experimental settings, which previously showed lack of effects of the behavioral testing alone on the measured parameters of stress, plasticity and neuroinflammation in mice (Couch et al., 
2013, 2016; Costa-Nunes et al., 2014; Vignisse et al., 2017; Gorlova et al., 2019).
Days 1-21
Day 22
Day 23

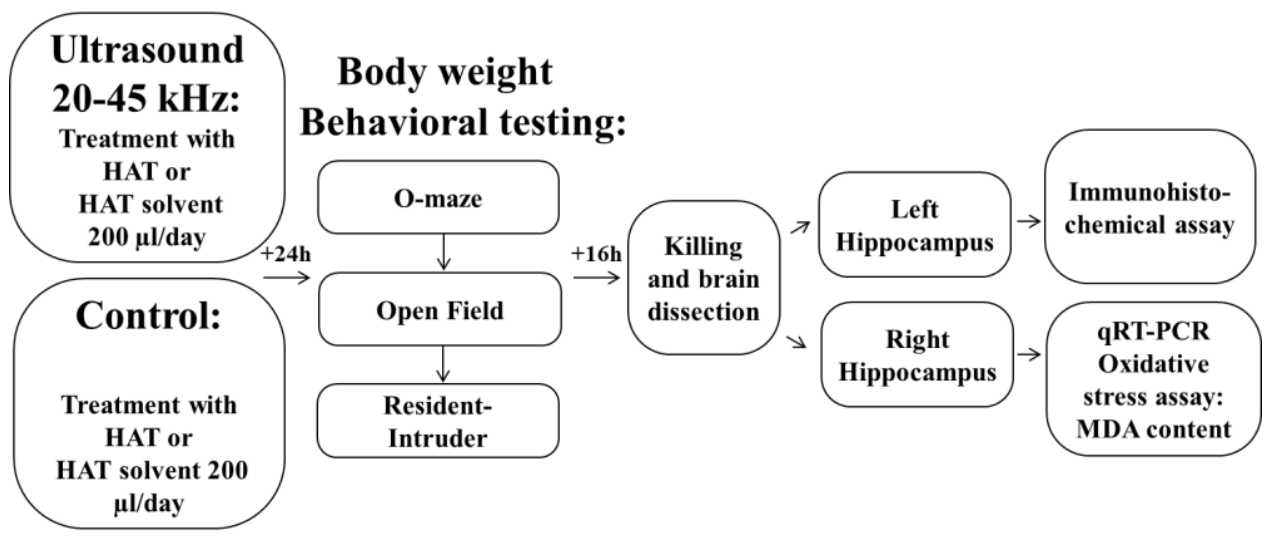

Figure 1. Experiment design. After 21-day of ultrasound exposure and dosing with herbal treatment (HAT) or HAT-solvent, mice were tested for behavior and then killed; their hippocampi were dissected for in vitro assays, including quantitative reverse transcription polymerase chain reaction (qRT-PCR) a measurement of malondialdehyde (MDA) content.

\subsection{Treatment administration}

Solutions of the herbal treatment (HAT) consisting of S-A and S-D drops, or its HAT-solvent (provided by Vendeville, Voorhout, The Netherlands; Supplementary Table 1) were administered orally using a pipette for 21 days concomitantly with ultrasound emission. Herbal treatment was dosed twice: administration of $30 \mu \mathrm{l}$ of S-A solution was followed by the administration of $90 \mu \mathrm{l}$ of S-D solution with a half-hour interval. In total, each HAT-treated mouse received $120 \mu \mathrm{l}$ of herbal treatment. Groups of mice treated with HAT-solvent, alcohol 
gluten-free based solution (Supplementary Table 1), received 120 $\mu \mathrm{l}$ of this solution. Concentrations of HAT ingredients were in a range of herbal treatments used alone or in herbal compositions used in human and in animal studies (see Supplementary Table 2).

\subsection{Ultrasound emission}

Ultrasound emission of average intensity of $50 \pm 5 \mathrm{~dB}$ and frequencies in a $20-45 \mathrm{~Hz}$ range was continuously delivered to the experimental groups of mice using a random schedule of alternating frequencies via a manufactured device (Weitech, Wavre, Belgium) and controlled by a detector (Discovery Channel, Crozet, VA, USA) as described elsewhere (Pavlov et al., 2019; Gorlova et al., 2019, see Supplementary material, Suppl.Fig.1). During the 10-min periods, ultrasound frequencies fluctuated at short time spans of $\leq 1 \mathrm{~s}$. $35 \%$ of the emission time consisted of 20 25 and $25-40 \mathrm{kHz}$ frequencies, and $30 \%$ of the emission time consisted of 40-45 kHz frequencies. The ultrasound device was suspended $2 \mathrm{~m}$ above the cages of the experimental groups with an average horizontal distance to cages of $2.5 \mathrm{~m}$. The position of the cages with respect to the stimulator was changed weekly. Applied ultrasound emission induced a loss of body weight and an increase of blood corticosterone concentrations in the experimental mice (Supplementary Fig.3) that demonstrates its stress impact. Previous validation studies suggested the selectivity of the adverse effects of applied low-frequency ultrasound versus the potential general negative action of a 
constant noise on behavioural correlates of "emotional state" in mice (Gorlova and Pavlov, personal communication). In particular, when mixed frequencies ("white noise") at the range of $20 \mathrm{~Hz}-20 \mathrm{kHz}$ were employed for 3 weeks, no changes in anxietylike behaviors in the elevated maze and open field were observed (Gorlova et al., personal communication).

\subsection{O-maze test}

The apparatus (Open Science, Russia), which consisted of a circular path (runway width $5.5 \mathrm{~cm}$, diameter $46 \mathrm{~cm}$ ), was placed $50 \mathrm{~cm}$ above the floor. Two opposing arms were protected by walls (height $10 \mathrm{~cm}$ ), and the illumination strength was $25 \mathrm{~lx}$. Anxietylike behavior was assessed using previously validated parameters (Couch et al., 2016; Strekalova et al., 2016). Mice were placed in

one of the closed arm compartments of the maze. The number of events of risk assessments, the number of exits to the open arms of the maze, total duration of time spent therein and the number of crossed sectors $(4 \times 5.5 \mathrm{~cm})$ were scored during $5 \mathrm{~min}$.

\subsection{Open field test}

In the open field model, mice were placed in a square gray plastic box $(45 \times 45 \times 45 \mathrm{~cm})$ illuminated with white light $(25 \mathrm{~lx})$ near the wall. The number of exploratory rearings, the number of grooming events, time spent in the central zone $(15 \times 15 \mathrm{~cm})$ and the number of crossed sectors $(11.25 \times 11.25 \mathrm{~cm})$ were scored for 10 min as described elsewhere (Strekalova et al., 2016; Pavlov et al., 2019). The recordings were subsequently analyzed using the 
automated video-tracking Viewpoint software (Viewpoint, Lyon, France).

\subsection{Resident-intruder test}

In the resident-intruder test, mice were individually placed in an observation cage $(30 \times 60 \times 30 \mathrm{~cm})$ for $30 \mathrm{~min}$. Then, each mouse was exposed to a previously group-housed naïve CD1 male intruder of similar weight and age for $4 \mathrm{~min}$ in the same cage. The latency to attack and their total number of attacks were scored as described elsewhere, number of attacking animals per group was calculated (Costa-Nunes et al., 2014; Strekalova et al., 2018; Gorlova et al., 2019).

\subsection{Brain Collection}

Mice were terminally anaesthetized with an intraperitoneal injection of Nembutal (Bayer, Wiesbaden, Germany) and then transcardially perfused with $10 \mathrm{ml}$ of the ice-cold $0.9 \% \mathrm{NaCl}$. Thereafter, right hippocampi of each mice were isolated via biopsy and immediately frozen at $-80^{\circ} \mathrm{C}$ on dry ice. The biopsy was performed by opening a section of the skull and cutting the hippocampus from above without damaging the basal arteries. After that, mice were transcardially perfused by $4 \%$ paraformaldehyde (PFA) in $0.1 \mathrm{M}$ phosphate buffer. Left hippocampi were removed and postfixed in $4 \%$-PFA for $12 \mathrm{~h}$ at $+4^{\circ} \mathrm{C}$, immersed in $0.1 \mathrm{M}$ phosphate-buffered saline (PBS, $\mathrm{pH}$ 7.4) for $12 \mathrm{~h}$ at $+4^{\circ} \mathrm{C}$, and processed for paraffin embedding; all procedures were carried out as described elsewhere (Pavlov et al., 
2019). $10 \mathrm{mg}$ of frozen tissue was separated for MDA assay, and the rest of the tissue was used for RT-PCR analysis.

2.9. Quantitative reverse transcription polymerase chain reaction analysis (qRT-PCR)

Quantitative real-time PCR (qRT-PCR) was carried out as described elsewhere (Couch et al., 2016; Pavlov et al., 2019). Briefly, total mRNA was isolated with TRI Reagent (Invitrogen, Carlsbad, CA, USA). 1 pg of total RNA was converted into cDNA using random primers and Superscript III transcriptase (Invitrogen, Carlsbad, CA, USA). qRT-PCR was performed in a 10 $\mu \mathrm{l}$ reaction volume containing a SYBR Green master mix (5 $\mu$ l, Bio-Rad Laboratories, Philadelphia, PA, USA). All qRT-PCR samples were ran on a CFX96 Deep Well Real-Time PCR Detection System (Bio-Rad, Hercules, CA, USA) in triplicate (see Supplementary material and Supplementary Table 3). The housekeeping gene glyceraldehyde-3-phosphate dehydrogenase (GAPDH) was selected as the reference following previously established protocols (Pavlov et al., 2019). Data were normalized to GAPDH mRNA expression and calculated as relative-fold changes compared to control mice as described elsewhere (Strekalova et al., 2016; Couch et al., 2016; Gorlova et al., 2019).

\subsection{Immunohistochemical analysis}

Immunohistochemical analysis was performed as described previously (Strekalova et al., 2018; Vignisse et al., 2017; Pavlov et al., 2019). Ki67 immunofluorescent staining was performed to 
identify proliferating cells; hexaribonucleotide binding protein-3 $(\mathrm{NeuN})$ immunofluorescent staining was used to label neurons, staining with DAPI was performed to visualize cell nuclei. Immunofluorescent staining of subunits of AMPA receptor was performed for subunits GluA1-GluA3. Numbers of surface puncta and of internalized puncta, and the internalization index were determined for each subunit. An internalization index was calculated by dividing the number of internalized puncta by the sum of internalized and surface numbers of puncta (see Supplementary material).

\subsection{Malondialdehyde assay}

Hippocampal malondialdehyde content was measured following Abcam ab118970 kit instructions (Abcam, Eugene, OR, USA; see Supplementary material). Briefly, the tissue was washed in cold PBS and homogenized in lysis solution, and then centrifuged at $13000 \mathrm{~g}$ for $10 \mathrm{~min}$. TBA reagent was added to supernatant and, after incubation at $95^{\circ} \mathrm{C}$ for $60 \mathrm{~min}$ and cooling to $\mathrm{RT}$ in an ice bath, supernatant was analyzed at $532 \mathrm{~nm}$ in a 96 well microplate as described elsewhere (Vignisse et al., 2017; Pavlov et al., 2019).

\subsection{Statistical analysis}

Data analysis was performed using GraphPad Prism software version 6.01 for Windows (GraphPad Software, La Jolla, CA, USA). Data were treated by a two-way ANOVA with Tukey's multiple comparison post-hoc test. Cohen's d for each significant finding to provide effect sizes was additionally calculated. The $\mathrm{x}^{2-}$ 
test was applied to compare the number of attacking vs nonattacking animals between the groups. Spearman test was used for correlation analysis. Statistical significance was set at $p<0.05$. Data are shown as mean \pm SEM.

\section{Results}

\subsection{Effects of ultrasound exposure and herbal} treatment in the $\mathrm{O}$-maze

ANOVA revealed a significant interaction between ultrasound exposure and herbal treatment for total number of events of risk assessments, total number of entries to the open arms of the O-maze, and total time spent by mice in the open arms $\left(\mathrm{F}_{2,25}=5.568, \mathrm{p}=0.0100 ; \mathrm{F}_{2,25}=7.405, \mathrm{p}=0.003 ; \mathrm{F}_{2,25}=8.852, \mathrm{p}=0.001\right.$, respectively, two-way ANOVA). All these parameters were significantly affected by stress $\left(\mathrm{F}_{1,25}=9.857, \mathrm{p}=0.004 ; \mathrm{F}_{1,25}=17.22\right.$, $\mathrm{p}<0.001 ; \quad \mathrm{F}_{1,25}=13.67, \mathrm{p}=0.001$, respectively) and by herbal treatment $\quad\left(\mathrm{F}_{2,25}=6.635, \quad \mathrm{p}=0.0091 ; \quad \mathrm{F}_{2,25}=3.718, \quad \mathrm{p}=0.039\right.$; $\mathrm{F}_{2,25}=10.20$, $\mathrm{p}<0.001$, respectively, two-way ANOVA; Fig.2A-C). Post hoc analysis revealed a significant decrease in the total number of entries to the open arms, the time spent in the open arms of the O-maze, and non-significant decrease in the total number of events of risk assessments in stressed non-treated mice in comparison with control non-treated mice ( $\mathrm{p}=0.003$ and $\mathrm{d}=3.71$; $\mathrm{p}=0.002$ and $\mathrm{d}=3.98 ; \mathrm{p}=0.066$, Tukey's and Cohen's $\mathrm{d}$ tests, respectively). There was a significant decrease in the total number of events of risk assessments, the total number of entries 
to open arms, and a non-significant decrease in the time spent in the open arms of the O-maze in stressed mice treated with HATsolvent in comparison with control non-treated mice $(p=0.033$, and $\mathrm{d}=2.69 ; \mathrm{p}=0.021$ and $\mathrm{d}=2.96 ; \mathrm{p}=0.073$, respectively) that suggested a lack of significant effects of the solvent on measured behavioural parameters of stressed mice. These parameters were significantly higher in stressed HAT-treated mice in comparison with stressed non-treated animals $(p=0.019$ and $d=1.96 ; p=0.002$ and $d=3.38 ; \mathrm{p}<0.001$ and $d=2.78$; Fig.2A-C). These data suggest increases of behavioral correlates of pro-anxiogenic changes in the ultrasound-exposed mice, their rescue by herbal treatment, and the lack of significant effects of its solvent on this behavior. No

differences were found between groups of control non-stressed mice, suggesting a lack of effects of the solvent and HAT in these animals.

We found no significant effects of stress $\left(\mathrm{F}_{1,24}=0.113\right.$, $\mathrm{p}=0.739$, two-way ANOVA), treatment $\left(\mathrm{F}_{2,24}=0.147, \mathrm{p}=0.863\right)$, or interaction $\left(\mathrm{F}_{2,24}=1.004, \mathrm{p}=0.381\right.$; Suppl. Fig.4A) on the number of crossed sections in the O-maze.

\subsection{Altered open field behavior in mice subjected to the ultrasound and effects of herbal treatment}

In the open field test, there was a significant interaction between ultrasound exposure and herbal treatment on the total number of rearings and the time spent in the center $\left(\mathrm{F}_{2,25}=0.247\right.$, $\mathrm{p}=0.105$ and $\mathrm{F}_{2,25}=20.58, \mathrm{p}<0.001$, respectively, two-way 
ANOVA), as well as significant stress and treatment effects $\left(\mathrm{F}_{1,25}=21.71, \mathrm{p}<0.001\right.$ and $\mathrm{F}_{2,25}=4.531, \mathrm{p}=0.0209, \quad \mathrm{~F}_{1,25}=15.03$, $\mathrm{p}<0.001$ and $\mathrm{F}_{2,25}=34.67, \mathrm{p}<0.001$, respectively). Post hoc analysis showed significant decrease in the number of rearings and the total time spent in center in stressed non-treated mice $(\mathrm{p}=0.031$ and $\mathrm{d}=2.45 ; \mathrm{p}=0.001$ and $\mathrm{d}=4.01$, respectively; Tukey's and Cohen's $d$ tests) and mice treated with HAT-solvent ( $\mathrm{p}=0.011$ and $\mathrm{d}=2.50 ; \mathrm{p}<0.001$ and $\mathrm{d}=3.70$, respectively) in comparison to control non-treated mice. This measures were increased in stressed HAT-treated mice in comparison with stressed nontreated animals $(\mathrm{p}=0.024$ and $\mathrm{d}=1.53 ; \mathrm{p}<0.001$ and $\mathrm{d}=5.49$; Fig.2D,E).

There was no significant interaction between effects of the ultrasound exposure and treatment on this parameter $\left(\mathrm{F}_{2,25}=0.8759, \mathrm{p}=0.3583\right.$, two-way ANOVA), although we found a significant effect of herbal treatment $\left(\mathrm{F}_{2,25}=5.311, \mathrm{p}=0.012\right)$ and stress $\left(\mathrm{F}_{2,25}=6.534, \mathrm{p}=0.005\right)$ on the number of grooming events. Stressed HAT-treated animals displayed a decrease in the number of grooming events in comparison with stressed nontreated group ( $\mathrm{p}<0.001$ and $\mathrm{d}=2.84$; Tukey's and Cohen's $\mathrm{d}$ tests; Fig.2F). Of note, solvent-treated stressed animals showed no increase in this behavior suggesting that solvent, which was an alcohol-based solution, might have a partial anti-stress effect.

We found no significant effect of stress $\left(\mathrm{F}_{1,24}=1.11\right.$, $\mathrm{p}=0.302$, two-way ANOVA), as well as no significant effect of treatment $\left(\mathrm{F}_{2,24}=1.064, \mathrm{p}=0.360\right)$, or interaction $\left(\mathrm{F}_{2,24}=0.0062, \mathrm{p}=0.939\right.$; 
Suppl. Fig.4B) on the number of crossed sections in the open field, that suggests unaltered locomotor activity in stressed animals. This findings rules out potential confounds in evaluation of anxiety like behaviors that can be associated with chronic stress exposure (Strekalova et al., 2005).

Together, these results suggest that ultrasound stress increases scores of behavioral correlates of anxiety-like behavior, suppresses exploratory activity, induces excessive grooming, a sign of stress response, and that herbal treatment but not its solvent counteract these changes.

\subsection{Effects of the ultrasound stress and herbal treatment on aggressive behaviour}

In the resident-intruder test, ANOVA revealed a significant interaction between ultrasound exposure and herbal treatment in latency to attack and the total number of attacks $\left(\mathrm{F}_{2,25}=13.00\right.$, $\mathrm{p}<0.001 ; \mathrm{F}_{1,25}=32.67, \mathrm{p}<0.001$, respectively). These parameters were significantly affected by stress $\left(\mathrm{F}_{1,25}=44.46, \quad \mathrm{p}<0.001\right.$; $\mathrm{F}_{1,25}=32.67, \mathrm{p}<0.001$, respectively, two-way ANOVA) and herbal treatment $\quad\left(\mathrm{F}_{2,25}=16.45, \quad \mathrm{p}<0.001 ; \quad \mathrm{F}_{2,25}=16.60, \quad \mathrm{p}<0.001\right.$, respectively). There was a significant decrease in latency to attack in non-treated stressed mice $(\mathrm{p}<0.001$ and $d=4.37$, Tukey's and Cohen's d tests) and in stressed mice treated with HATsolvent in comparison with control non-treated mice ( $p<0.001$ and $\mathrm{d}=4.13)$ Stressed $\quad$ HAT-treated animals displayed decreased latency as compared to stressed non-treated 
group ( $p<0.001$ and $d=4.75$; Fig.2G). Non-treated stressed mice also displayed a significant increase in the number of attacks in comparison with control non-treated mice $(\mathrm{p}<0.001$ and $\mathrm{d}=4.16)$, while no such changes were observed for stressed HAT-treated animals $(\mathrm{p}=0.147)$ that also showed significantly smaller values of this measure, compared to the former group $(p<0.001$ and $\mathrm{d}=3.81$, Fig. $2 \mathrm{H})$.

The $x^{2}$ test showed a significant increase in number of attacking animals in non-treated stressed group and in stressed group treated with HAT-solvent in comparison with non-treated control group $(\mathrm{T}=4.286, \mathrm{p}=0.0384$ and $\mathrm{T}=4.286, \mathrm{p}=0.0384$, respectively). This measure was significantly decreased in the HAT-treated stressed group in comparison with non-treated stressed group $\left(\mathrm{T}=7.639, \mathrm{p}=0.0057, \mathrm{x}^{2}\right.$ test). Interestingly, solvent-treated stressed animals showed no significant increase in the number of attacks suggesting that solvent had partially prevented stress-induced augmentation of aggressive behavior. Together, behavioral correlates of aggressive behavior were increased in mice exposed to ultrasound that did not receive any treatment, but not in the stressed HAT-treated animals. 


\section{O-MAZE}

A
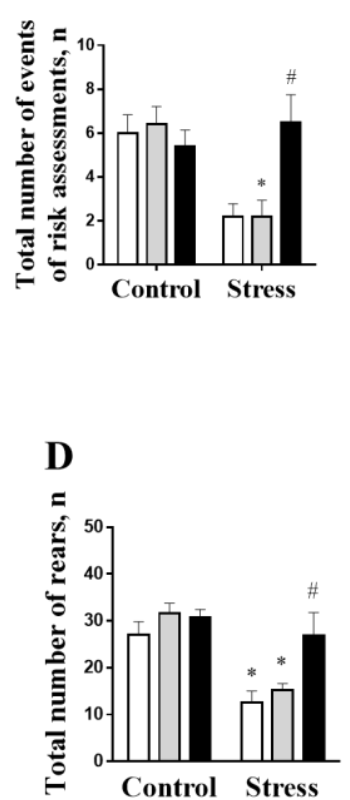

B

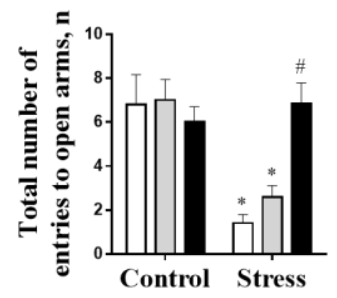

OPEN FIELD
C

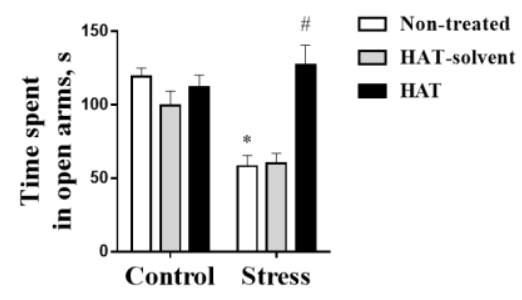

$\mathbf{E}$

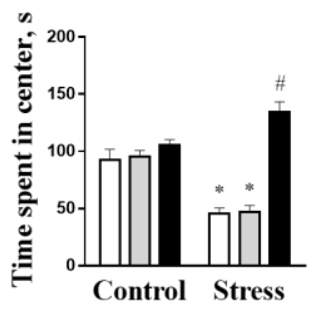

$\mathbf{F}$

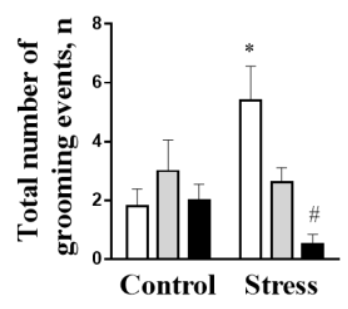

\section{RESIDENT-INTRUDER}

G

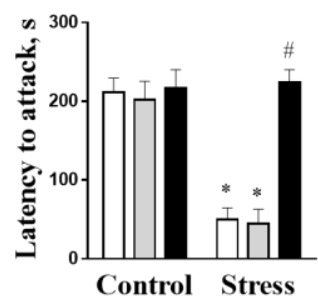

H

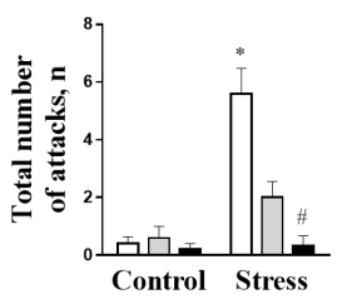

Figure 2. Behavioral changes in the ultrasound-exposed mice and effects of herbal treatment. In the O-maze test, in comparison to control non-treated group, stressed non-treated group and mice treated with HAT-solvent showed a significant decrease in (A) the total number of events of risk assessments (B) total number of entries to open arms and (C) time spent in open arms. Stressed HAT-treated animals did not differ from control non-treated mice in these measures. In the open field test, in comparison with control non-treated mice, stressed non-treated 
mice and stressed mice treated with HAT-solvent showed significant decreases in (D) total number of rears and (E) time spent in the center and (F) a significant increase in the number of grooming events. Stressed HAT-treated animals showed no such differences. In the resident-intruder test, in comparison with control non-treated group, non-treated stressed mice and stressed mice treated with HAT-solvent showed (G) a significant decrease of latency to attack and (H) a significant increase in the total number of attacks. Again, no such changes were found in stressed animals that received herbal treatment. ${ }^{*} \mathrm{p}<0.05$ vs. non-treated control group, $\# p<0.05$ vs. non-treated stressed group, two-way ANOVA and post hoc Tukey's test. 6-8 animals per group were used. HAT - herbal antioxidant treatment. Bars are Mean \pm SEM.

\subsection{Ultrasound exposure changes expression and} internalization of subunits of the AMPA receptor in the hippocampus: effects of herbal treatment

ANOVA revealed no significant interaction between the effects of stress and herbal treatment on mRNA expression of GluA1-A3 subunits $\left(\mathrm{F}_{2,25}=1.298, \mathrm{p}=0.230 ; \mathrm{F}_{2,25}=2.301, \mathrm{p}=0.121\right.$; $\left.\mathrm{F}_{2,25}=0.2, \mathrm{p}=0.820\right)$. At the same time, there was a significant effect of stress on mRNA expression of GluA1-A3 subunits $\left(\mathrm{F}_{1,25}=11.85, \mathrm{p}=0.002 ; \mathrm{F}_{1,25}=9.167, \mathrm{p}=0.006 ; \mathrm{F}_{1,25}=18.64, \mathrm{p}<0.001\right.$, respectively, two-way ANOVA) and no significant effect of herbal treatment $\left(\mathrm{F}_{2,25}=3.11, \mathrm{p}=0.062 ; \mathrm{F}_{2,25}=2.93, \mathrm{p}=0.072 ; \mathrm{F}_{2,25}=2.048\right.$, $p=0.150$, respectively). Non-significant decrease in the expression of GluA1 and GluA3 subunits was found in non-treated stressed mice $(p=0.102 ; p=0.099$, Tukey's test) and in stressed mice treated with HAT-solvent $(\mathrm{p}=0.180 ; \mathrm{p}=0.119$; Table 1$)$ in comparison with control non-treated mice. There was a significant increase of 
GluA2 expression in stressed non-treated mice in comparison with control non-treated mice ( $\mathrm{p}=0.038$, and $\mathrm{d}=1.93$, Tukey's and Cohen's $d$ tests), but not in mice treated with HAT $(p=0.447)$ or with HAT-solvent $(\mathrm{p}=0.102)$.

ANOVA revealed a significant stress and treatment interaction on number of internalized GluA1 and GluA2, but not GluA3 puncta $\left(\mathrm{F}_{2,25}=9.115, \quad \mathrm{p}=0.001 ; \quad \mathrm{F}_{2,25}=4.264, \quad \mathrm{p}=0.0260\right.$; $\mathrm{F}_{2,25}=0.807$, $\mathrm{p}=0.458$, respectively). There was a significant effect of stress on number of internalized GluA1, GluA2 and GluA3 puncta $\left(\mathrm{F}_{1,25}=22.70, \mathrm{p}<0.001 ; \mathrm{F}_{1,25}=29.94, \mathrm{p}<0.001 ; \mathrm{F}_{1,25}=13.54\right.$, $\mathrm{p}=0.001$, respectively, two-way ANOVA), as well as significant effect of herbal treatment on these measures $\left(\mathrm{F}_{2,25}=6.070\right.$, $\mathrm{p}=0.007 ; \mathrm{F}_{2,25}=6.965, \mathrm{p}=0.004 ; \mathrm{F}_{2,25}=4.682, \mathrm{p}=0.027$, respectively). Number of internalized GluA1-A3 puncta were increased in stressed non-treated mice in comparison with control non-treated mice $(\mathrm{p}<0.001$ and $\mathrm{d}=2.93 ; \mathrm{p}<0.003$ and $\mathrm{d}=2.36 ; \mathrm{p}=0.043$ and $d=1.67$, respectively, Tukey's and Cohen's d tests, Table 2). In stressed mice treated with HAT-solvent, in comparison with control non-treated group, number of internalized GluA2 puncta were significantly increased $(\mathrm{p}=0.040$ and $\mathrm{d}=2.65)$, while number of internalized GluA1 and Glua3 puncta was not significantly different from non-stressed controls $\quad(p=0.057 ; \mathrm{p}=0.067$, respectively). HAT-treated stressed mice displayed a decrease of number of internalized GluA1 and GluA2, but not GluA3 puncta in comparison to stressed non-treated group $(\mathrm{p}<0.001$ and $\mathrm{d}=3.35$; $\mathrm{p}=0.001$ and $\mathrm{d}=2.78 ; \mathrm{p}=0.114$, respectively; Fig.3A,C,E). No 
changes in number of internalized GluA1 and GluA3 puncta were found in non-stressed mice treated with HAT-solvent, while above-presented data suggest similar to HAT effects of the solvent on AMPA receptor sub-units expression and internalization during stress.

ANOVA revealed no significant effect of stress and herbal treatment interaction on number of surface GluA1, GluA2 and GluA3 puncta $\quad\left(\mathrm{F}_{2,25}=0.623, \quad \mathrm{p}=0.545 ; \quad \mathrm{F}_{2,25}=2.783, \quad \mathrm{p}=0.082\right.$; $\mathrm{F}_{2,25}=4.039, \mathrm{p}=0.08$, respectively). There was a significant effect of stress on number of surface GluA1-A3 puncta $\left(\mathrm{F}_{1,25}=18.45\right.$, $\mathrm{p}<0.001 ; \mathrm{F}_{1,25}=6.649, \mathrm{p}<0.017 ; \mathrm{F}_{1,25}=5.928, \mathrm{p}=0.023$, respectively). There was a non-significant decrease in surface GluA1-A3 puncta in non-treated stressed mice $(\mathrm{p}=0.073 ; \mathrm{p}=0.190 ; \mathrm{p}=0.14$, respectively, Tukey's test; Fig.3B,D,F and Table 3) and in stressed mice treated with HAT-solvent $(\mathrm{p}=0.067, \mathrm{p}=0.168$; $\mathrm{p}=0.169$, respectively), in comparison with control non-treated mice. Herbal treatment significantly affected number of surface GluA2 and GluA3 puncta $\left(\mathrm{F}_{2,25}=4.98, \mathrm{p}=0.016 ; \mathrm{F}_{2,25}=5.992\right.$, $\mathrm{p}=0.008$, respectively), but not GluA1 puncta $\left(\mathrm{F}_{2,25}=1.229\right.$, $\mathrm{p}=0.310$ ). Specifically, number of surface GluA2 and GluA3, but not GluA1 puncta were significantly increased in stressed HATtreated mice in comparison with stressed non-treated mice ( $\mathrm{p}=0.036$ and $\mathrm{d}=2.32 ; \mathrm{p}=0.007$ and $\mathrm{d}=2.78, \mathrm{p}=0.341$, respectively). No changes in these measures were found in HAT-treated nonstressed mice. 
ANOVA revealed a significant interaction between the ultrasound stress and the herbal treatment in GluA1, GluA2, and GluA3 internalization index of subunits $\left(\mathrm{F}_{2,24}=12.19\right.$, $\mathrm{p}=0.0002$; $\mathrm{F}_{2,24}=9.704, \mathrm{p}=0.0008$ and $\mathrm{F}_{2,24}=4.926, \mathrm{p}=0.0157$, respectively, two-way ANOVA). We also found a significant stress effect $\left(\mathrm{F}_{1,24}=72.02, \mathrm{p}<0.0001 ; \mathrm{F}_{1,24}=26.93, \mathrm{p}<0.0001\right.$ and $\mathrm{F}_{1,24}=6.808$, $\mathrm{p}=0.0151$, respectively) and significant effect of the herbal treatment $\left(\mathrm{F}_{2,24}=3.784, \mathrm{p}=0.0367 ; \quad \mathrm{F}_{2,24}=7.408, \mathrm{p}=0.003\right.$ and $\mathrm{F}_{2,24}=6.808, \mathrm{p}=0.003$, respectively) on the internalization index. Post hoc analysis revealed a significant increase in the internalization index of GluA1, GluA2, and GluA3 in stressed non-treated mice in comparison with non-treated controls $(\mathrm{p}<0.0001$ and $\mathrm{d}=2.12, \mathrm{p}=0.003$ and $\mathrm{d}=1.14, \mathrm{p}=0.0419$ and $\mathrm{d}=1.02$, respectively, Tukey's and Cohen's d tests; Table 4). There was also non-significant decrease of the internalization index of GluA1 and GluA2, but not of GluA3 subunit in stressed mice treated with HAT-solvent in comparison with non-treated controls $(p=0.0001$ and $d=2.02, p=0.0002$ and $d=1.93, p=0.157$ and $d=1.13$, respectively). Internalization index was significantly decreased in stressed HAT-treated mice in comparison to stressed non-treated group for all subunits $(\mathrm{p}=0.0001$ and $\mathrm{d}=2.34, \mathrm{p}<0.0001$ and $\mathrm{d}=2.16, \mathrm{p}=0.012$ and $\mathrm{d}=1.09$, respectively). Thus, ultrasound exposure increased internalization of GluA1-A3 subunits of hippocampal AMPA receptor. Herbal treatment precluded these effects of the ultrasound stress in not-treated or HAT-solvent- 
treated animals and increased surface GluA2 and GluA3 subunits.

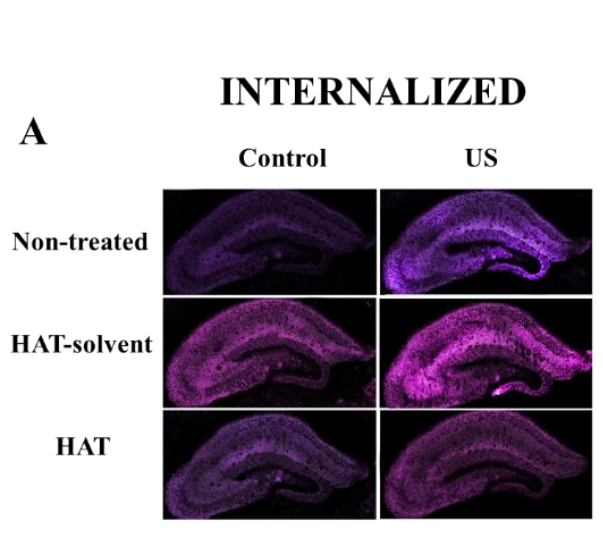

\section{GluA1}

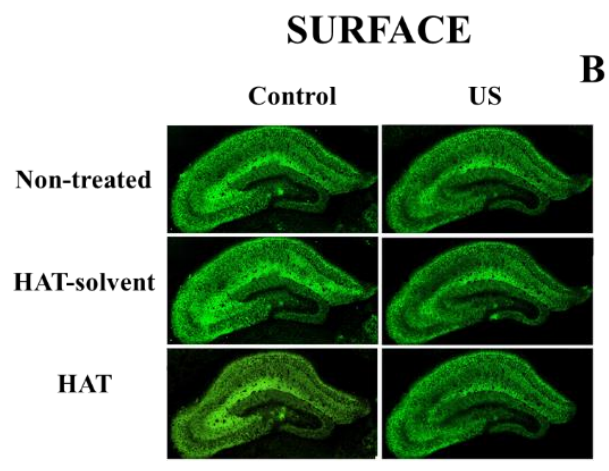

C

GluA2
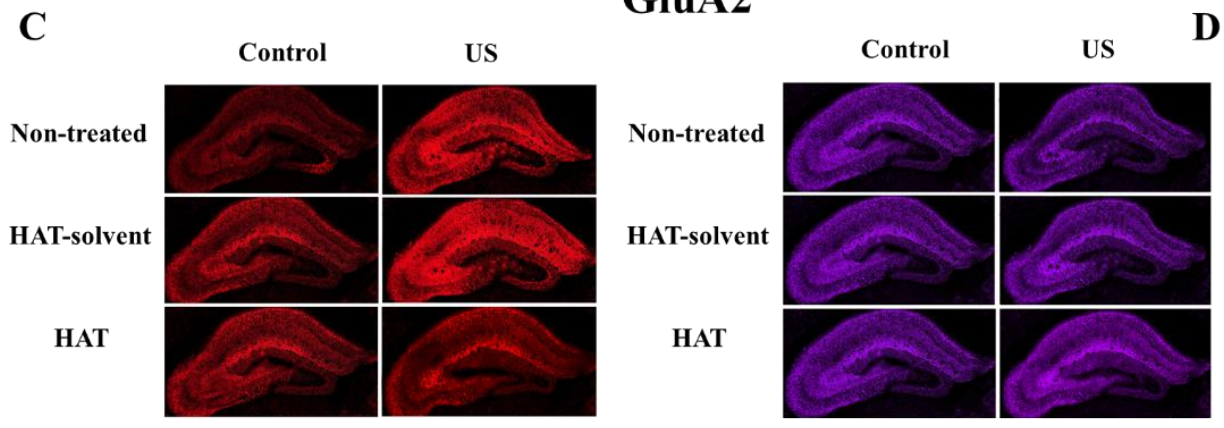

$\mathbf{E}$

\section{GluA3}
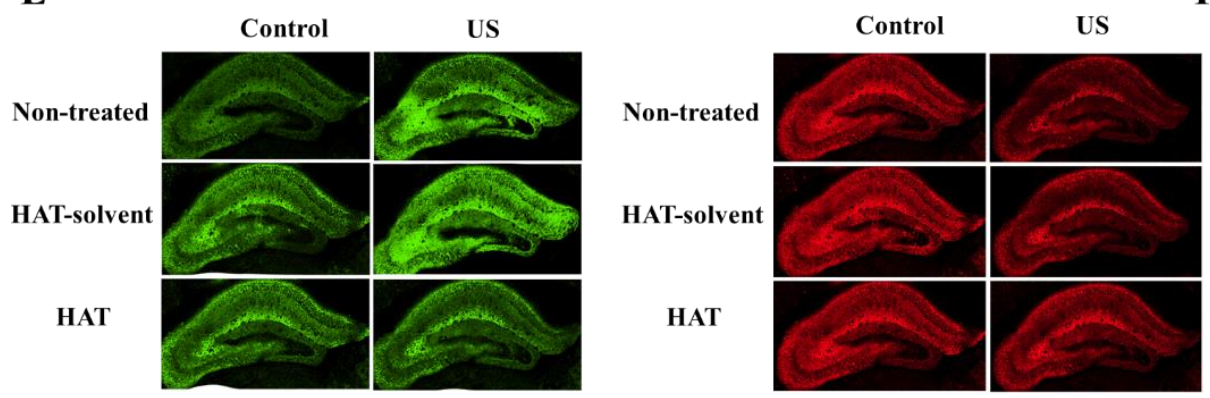
Figure 3. Immunohistochemical analysis of surface and internalized GluA subunits. Immunohistochemical analysis of (A) internalized GluA1, (C) GluA2 and (E) GluA3 subunits and surface (B) GluA1, (D) GluA2 and (F) GluA3 subunits of AMPA receptor.

\subsection{Ultrasound exposure and herbal treatment affect} hippocampal expression of cytokines, GSK3ß, Akt and number of Ki67-positive cells

We found significant increases in pro-inflammatory cytokine expression. There was no significant effect of stress and treatment interaction on TNF mRNA expression $\left(\mathrm{F}_{2,25}=3.36\right.$, $\mathrm{p}=0.051$, two-way ANOVA). ANOVA revealed a significant effect of stress $\left(\mathrm{F}_{1,25}=28.41, \mathrm{p}<0.001\right)$ and herbal treatment $\left(\mathrm{F}_{2,25}=3.395\right.$, $\mathrm{p}=0.049)$ on this measure, which was increased in stressed nontreated mice $(p=0.027$ and $d=3.20$, Tukey's and Cohen's $d$ test) and in stressed mice that received HAT-solvent in comparison with control non-treated mice ( $p=0.001$ and $d=2.76$; Fig.4A).

We found a significant interaction between ultrasound exposure and herbal treatment on GSK-3B and IL-1B mRNA levels $\left(\mathrm{F}_{2,25}=3.999, \mathrm{p}=0.031 ; \mathrm{F}_{2,25}=5.860, \mathrm{p}=0.008\right.$, respectively, two-way ANOVA). This measures were significantly altered by stress $\left(\mathrm{F}_{1,25}=45.87, \mathrm{p}<0.001 ; \mathrm{F}_{1,25}=32.48, \mathrm{p}<0.001\right.$, respectively) and herbal treatment $\left(\mathrm{F}_{2,25}=6.104, \mathrm{p}=0.007 ; \mathrm{F}_{2,25}=4.088, \mathrm{p}=0.029\right.$, respectively, Fig.4B,C). In comparison with control non-treated mice, GSK-3B and IL-1B mRNA levels were increased in stressed non-treated mice $(\mathrm{p}<0.001$ and $\mathrm{d}=2.75 ; \mathrm{p}<0.001$ and $\mathrm{d}=3.45$, respectively, Tukey's and Cohen's d tests) and stressed mice that 
received HAT-solvent $(\mathrm{p}=0.002$ and $\mathrm{d}=2.45 ; \mathrm{p}=0.002$ and $\mathrm{d}=2.16$, respectively). There was a significant decrease in these parameters in stressed HAT-treated mice in comparison with stressed non-treated mice $(\mathrm{p}=0.002$ and $\mathrm{d}=2.48 ; \mathrm{p}=0.006$ and d=3.31, respectively; Fig.4B,C).

ANOVA revealed no significant stress and treatment interaction on mRNA levels IL-6, IL-15 and Akt $\left(\mathrm{F}_{2,25}=2.950\right.$, $\mathrm{p}=0.071 ; \mathrm{F}_{2,25}=0.02, \mathrm{p}=0.979 ; \mathrm{F}_{2,25}=0.433, \mathrm{p}=0.653$, respectively, two-way ANOVA) in the hippocampus. Stress significantly increased mRNA concentrations of IL- 6 and Akt, but not IL-15 mRNA levels in the hippocampus $\left(\mathrm{F}_{1,25}=35.66, \quad \mathrm{p}<0.001\right.$; $\mathrm{F}_{1,25}=6.250, \quad \mathrm{p}=0.019 ; \quad \mathrm{F}_{1,25}=0.593, \quad \mathrm{p}=0.449, \quad$ respectively). Furthermore, ANOVA revealed no significant effect of herbal treatment on these measures $\left(\mathrm{F}_{2,25}=1.357, \mathrm{p}=0.276 ; \mathrm{F}_{2,25}=0.1791\right.$, $\mathrm{p}=0.837 ; \mathrm{F}_{2,25}=0.846, \mathrm{p}=0.441$, respectively). Stressed nontreated group displayed significantly increased IL-6 mRNA levels in comparison with control non-treated mice $(\mathrm{p}<0.001$ and $\mathrm{d}=1.32$, Tukey's and Cohen's d tests, Fig.4D). At the same time, post hoc analysis did not reveal significant differences in HAT-treated stressed mice in this measure $(\mathrm{p}=0.363 ; \mathrm{p}=0.567$, Tukey's test, respectively). Stressed mice treated with HAT-solvent showed no changes in IL-6 mRNA level in comparison with control nontreated animals ( $\mathrm{p}=0.978, \mathrm{p}=0.786$; Fig. $4 \mathrm{D}$ ) suggesting partial anti-inflammatory action of the solvent, which might underlie its effects on behavioral parameters. 
A TNF

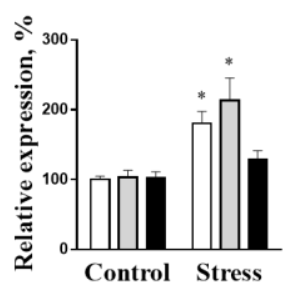

D IL-6

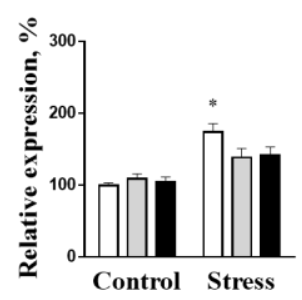

G $\quad \mathbf{K i 6 7}$

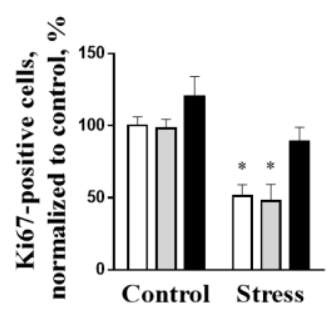

B GSK-3beta

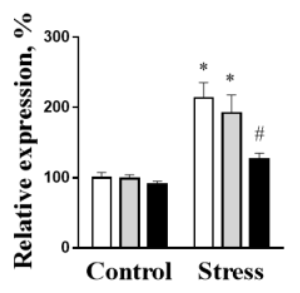

E IL-15

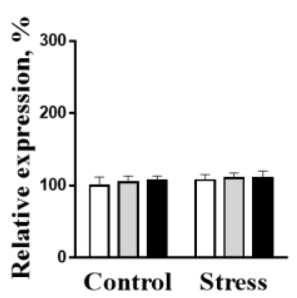

C IL-1beta

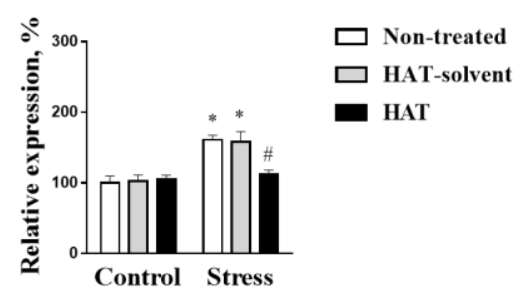

F AKT

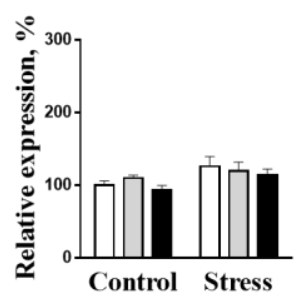

Figure 4. Ultrasound exposure and herbal treatment effect on expression of cytokines, GSK3B, Akt and number of Ki67-positive cells in the hippocampus. Stressed non-treated mice and stressed mice treated with HAT-solvent displayed a significant increase of (A) TNF mRNA expression, (B) GSK-36 mRNA expression, (C) IL-1B mRNA expression and (D) IL-6 mRNA expression in the hippocampus, in comparison with control non-treated mice. (E) There were no significant differences between groups in IL-15 mRNA (F) and in Akt mRNA concentrations. (G) Stressed non-treated mice and stressed mice treated with HAT-solvent displayed a significant decrease in Ki67positive cells in the hippocampus that was not observed in HAT-treated 
group subjected to stress. ${ }^{*} \mathrm{p}<0.05$ vs. non-treated controls, $\# \mathrm{p}<0.05$ vs. non-treated stressed group, two-way ANOVA and post hoc Tukey's test. 6-8 animals per group were used. HAT - herbal antioxidant treatment. Bars are Mean \pm SEM.

There was no significant interaction between stress in HAT treatment in their effects on the number of Ki67-positive cells, a marker of neuronal proliferation $\left(\mathrm{F}_{2,24}=0.607, \mathrm{p}=0.553\right.$, two-way ANOVA). This parameter was significantly affected by stress $\left(\mathrm{F}_{1,24}=30.38, \quad \mathrm{p}<0.001\right)$ and herbal treatment $\left(\mathrm{F}_{2,24}=6.603\right.$, $\mathrm{p}=0.005$ ). Post hoc analysis revealed a decrease in the number of Ki67-positive cells in stressed non-treated mice $(p=0.017$ and $\mathrm{d}=3.18$, Tukey's and Cohen's d tests) and in stressed mice that received HAT-solvent in comparison with control non-treated mice ( $p=0.013$ and $d=2.79$, Fig.4G). We found significant effect of stress $\left(\mathrm{F}_{1,24}=9.701, \mathrm{p}=0.005\right)$, but not treatment $\left(\mathrm{F}_{2,24}=0.159\right.$, $\mathrm{p}=0.854)$, nor interaction $\left(\mathrm{F}_{2,24}=0.939, \mathrm{p}=0.405\right.$, two-way ANOVA, Suppl. Fig.6) on the number of NeuN-positive cells. Post hoc analysis did not reveal significant changes in stressed nontreated mice and stressed mice treated with HAT-solvent in comparison with control $(\mathrm{p}=0.2247 ; \mathrm{p}=0.1978$, respectively, Tukey's test). Thus, ultrasound exposure resulted in the decreased number of Ki67-positive cells, but did not affect the number of NeuN-positive cells. 


\subsection{Elevated hippocampal malondialdehyde content}

correlates with neurobiological effects of ultrasound stress: effects of herbal treatment

We found a significant interaction between stress and treatment in the effects on MDA content $\left(\mathrm{F}_{2,25}=4.360\right.$, $\left.\mathrm{p}=0.024\right)$. There was a significant effect of stress $\left(\mathrm{F}_{1,25}=23.16, \mathrm{p}<0.001\right)$ and herbal treatment $\left(\mathrm{F}_{2,25}=4.556, \mathrm{p}=0.02\right)$ on the MDA levels in the hippocampus. Post hoc analysis revealed elevated hippocampal MDA content in stressed non-treated mice ( $\mathrm{p}=0.033$ and $\mathrm{d}=1.90$, Tukey's and Cohen's d tests) and stressed mice that received HAT-solvent in comparison with control non-treated group $(p=0.002$ and $d=2.16)$. There was a significant decrease in this parameter in stressed HAT-treated mice in comparison with stressed non-treated mice ( $\mathrm{p}=0.034$ and $\mathrm{d}=2.09$, Fig.5A).

Correlation analysis performed in all experimental groups reveal significant correlations between hippocampal MDA levels and the most of investigated end-points. Significant correlations were found between the MDA content and the total number of entries to the open arms of the O-maze ( $\mathrm{p}=0.002, \mathrm{r}=-0.532)$, the time spent therein $(\mathrm{p}<0.001, \mathrm{r}=-0.573)$, the total number of rearings $(p<0.001, r=-0.632)$, the time spent in the center of the open field $(p<0.001, r=-0.635)$, latency to attack $(p<0.001, r=$ 0.734 ), the total number of attacks ( $\mathrm{p}=0.013, \mathrm{r}=0.551$; Fig.5B-G). There were significant correlations between the MDA content and mRNA levels of GluA2 ( $\mathrm{p}=0.001, \mathrm{r}=0.551)$, TNF $\quad(\mathrm{p}<0.001$, $r=0.807), I L-1 B(p<0.001, r=0.649)$, and GSK3B $(p=0.002, r=0.543$; 
Fig.5H-K). Finally, we found significant correlations between the MDA content and internalized GluA1 puncta ( $<<0.001, \mathrm{r}=0.639)$, internalized GluA2 puncta $(\mathrm{p}=0.002, \mathrm{r}=0.569)$ and number of Ki67-positive cells ( $<<0.001, r=-0.56$; Fig.5L-N). Isolated analysis of the above-described correlations showed significant correlations of MDA content with most of the indicated parameters in stressed, but not in non-stressed groups analyzed (see Supplementary material, Suppl. Figs.7,8). This further highlights a relationship between ultrasound stress-induced neurobiological changes and oxidative stress.

\section{Discussion}

Exposure to ultrasound stress resulted in elevated scores of correlates of anxiety-like behaviour in the non-treated group of mice. These changes were accompanied by suppressed vertical / exploratory activity, augmented aggression scores, grooming behavior, hippocampal expression of the AMPA receptor subunit GluA2, and of internalization rate of GluA1-A3 subunits of this receptor. We observed an increase in hippocampal expression of the investigated pro-inflammatory cytokines Il-1B, Il-6, TNF, as well as of GSK3B, and elevated malondialdehyde content. The number of Ki67-positive cells, a marker of cellular proliferation, was reduced in stressed non-treated animals. Chronic dosing with herbal antioxidant/anti-inflammatory treatment prevented most of these changes. This study revealed a strong correlation between the hippocampal malondialdehyde levels on one hand, 
and behavioral and molecular changes induced by ultrasound stress on another hand. These correlations were only present in stressed, but not in non-stressed mice which further highlights the role of oxidative stress in neurobiological abnormalities, including behavioural correlates of elevated anxiety associated with a state of "emotional" stress.

Behavioral parameters of anxiety-like state in the O-maze were elevated in non-treated animals exposed to ultrasound. This finding was further supported by the results obtained in the open field test and is in line with previous findings in this paradigm (Pavlov et al., 2019). Increase in grooming behavior and decrease in vertical activity in stressed non-treated mice observed in this study can be a sign of chronic stress in small rodents and is associated with behavioral stereotypy and suppressed novelty exploration, respectively (Kalueff, 2006; Strekalova et al., 2004). Augmented aggression scores observed in the present study are consistent with previous results obtained in the ultrasound stress model (Gorlova et al., 2019; Pavlov et al., 2019) and are generally supported by the literature demonstrating a relationship between increases in behavioural parameters of stress, anxiety and aggression both in animal and clinical studies (Bandelow and Michaelis 2015; Chen et al., 2013). A lack of changes in general locomotion rules out a possibility of confounds that potentially can result from prolonged exposure to stress (Strekalova et al., 2005), in behavioral analysis of mice. 
A

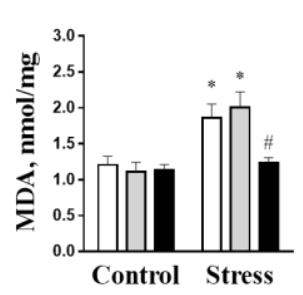

D

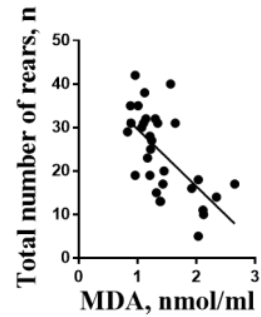

H

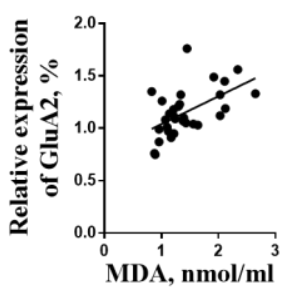

$\mathbf{L}$

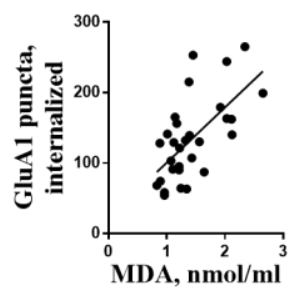

B

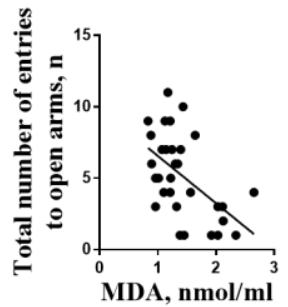

$\mathbf{E}$

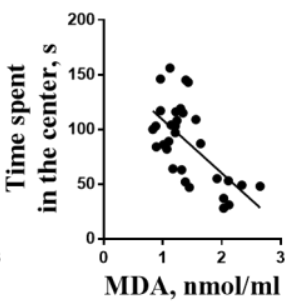

I

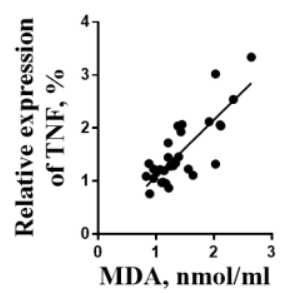

M

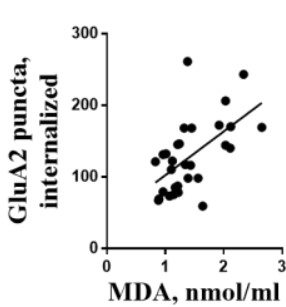

C
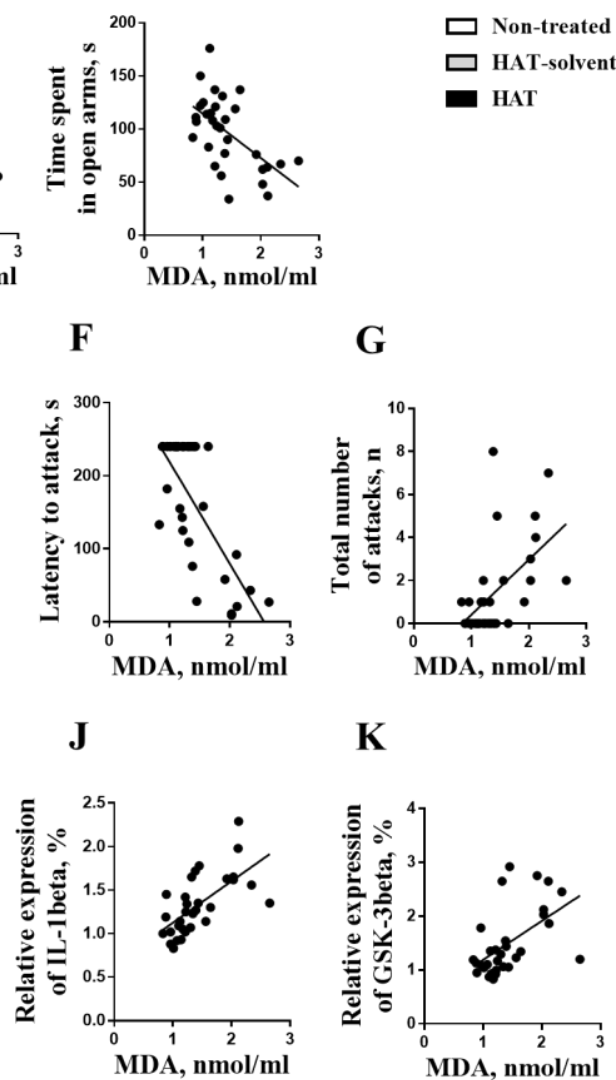

$\mathbf{F}$

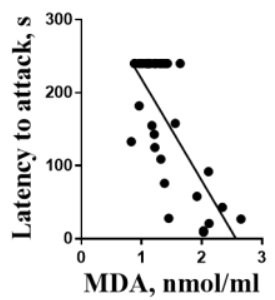

$\mathbf{J}$

G

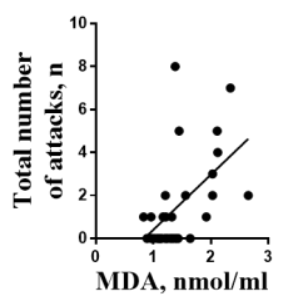

K

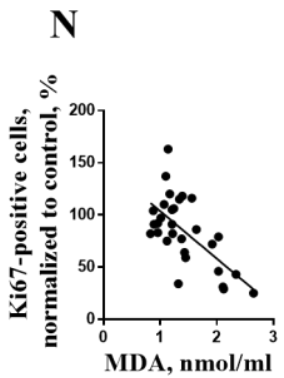

Figure 5. Elevated hippocampal malondialdehyde content correlates with neurobiological effects of ultrasound stress: effects of herbal treatment. (A) Stressed non-treated mice and stressed mice treated with HAT-solvent displayed a significant increase in MDA content in the hippocampus. Stressed HAT-treated animals did not differ from control non-treated mice in this parameter and displayed a 
significantly decreased MDA levels in comparison with stressed nontreated group. Significant correlations were found between the malondialdehyde content and (B) total number of entries to open arms of the O-maze, (C) time spent in open arms of the O-maze, (D) total number of rears, (E) time spent in the center of the open field, (F) latency to attack, (G) total number of attacks, (H) GluA2 mRNA expression, (I) TNF mRNA expression, (J) IL-1B mRNA expression, (K) GSK-38 mRNA expression, (L) GluA1 internalized puncta, (M) GluA2 internalized puncta, $(\mathrm{N})$ number of Ki67-positive cells. ${ }^{*} \mathrm{p}<0.05$ vs. nontreated controls, $\# \mathrm{p}<0.05$ vs. non-treated stressed group, two-way ANOVA and post hoc Tukey's test; Spearman correlation. 6-8 animals per group were used. HAT - herbal antioxidant treatment. Bars are Mean \pm SEM.

Emotional-like abnormalities induced by ultrasound in mice were accompanied by altered gene expression of the AMPA receptor subunits, suggesting their dysregulation, in the hippocampus. A dysregulation of these processes in the brain was previously shown to be related with increased signs of anxiety (Weber et al., 2015), aggression (Adamczyk et al, 2012) and impaired recovery from stress (Martinowich et al, 2012). We found an increased gene expression of GluA2 subunit and a nonsignificant decrease of GluA1 and GluA3 subunits expression, which is in line with previous studies (Gorlova et al., 2019). Other stress experiments, e.g. with mouse unpredictable chronic stress paradigm, revealed up-regulated GluA2 expression in the brain associated with signs of increased anxiety-like and aggressive behaviors (Costa-Nunes et al., 2014).

Immunohistochemical analysis of expression of internalized and surface GluA1-3 subunits uncovered an elevated rate of 
internalization of subunits of all investigated AMPA receptor subunits in the hippocampus in stressed non-treated mice. The internalization of the AMPA receptor subunits plays a key role in the modulation of receptor bioavailability, and thus, functioning, as this process terminates receptor cell surface signaling and designates activated receptors for degradation (Pradhan et al., 2016; van Rijnsoever et al., 2005). The internalization regulates cell-surface expression of the AMPA receptor in neurons (van Rijnsoever et al., 2005). In the present study, increased internalization and the increased ratio of internalized / surface AMPA receptor subunits may suggest an adaptive decrease in the glutamate-mediated neurotransmission via GluA1-3 subunits. This, in turn, can dampen the negative effects of glutamatergic excitotoxicity, an important pathogenetic factor of stress (Caudal et al., 2010; Bonini et al., 2016). The reported increase in the mRNA levels of GluA2 may be a sign of compensatory changes triggered by downregulation of receptor function. In this context, lack of similar increases in gene expression of GluA1 and GluA3 subunits might be explained by different dynamics of these regulatory processes.

Thus, present findings suggest a suppressive effect of ultrasound stress on the AMPA receptor-mediated signaling and synaptic plasticity that can underlie stress-related emotional abnormalities (Bowers et al., 2010) and explain reported here behavioral changes. Chronic stress can impair adult neurogenesis (Schoenfeld and Gould, 2012; Lajud et al., 2012; Vignisse et al., 
2017), and reduced number of Ki67-positive cells in the hippocampus serves as further evidence for suppressed neuronal plasticity induced by ultrasound exposure. This finding is in line with previously reported decreases in gene and protein expression of plasticity regulators PSD95, PSA-NCAM, B-catenin (Gorlova et al., 2019) and density of Ki67- positive cells in the subgranular zone of the dentate gyrus of the hippocampus (Pavlov et al., 2019).

We found an increased hippocampal expression of proinflammatory cytokines IL-1B, IL-6 and TNF, that supports our previous observations. On another hand, the expression of the anti-inflammatory cytokine IL-15 was unaltered. As neuroinflammation was shown to accompany pathological anxiety (Salim, 2017; Rossi et al., 2017) and since an increase in the synthesis of brain pro-inflammatory cytokines in mice can promote anxiogenic-like (Chen et al, 2013) and aggressive behaviors (Takahashi et al, 2018), present data implicate the reported here over-production of pro-inflammatory cytokines in the anxiogenic effects of ultrasound stress. We also demonstrated that in the ultrasound-exposed mice, elevated scores of anxietylike behaviors were associated with the over-expression of GSK38 , that governs the production of pro-inflammatory cytokines (Green and Nolan, 2012), affects oxidative stress (Potz et al., 2018) and regulates hippocampal cellular plasticity (Peineau at al., 2008). In current study, the expression of its physiological antagonist Akt (Manning and Toker, 2017), was unaltered. 
The use of hippocampi from the same animals for the variety of assays employed here made it possible to perform the correlation analysis between the pro-oxidative changes and other molecular end-points in addition to behavioral parameters. Most of the investigated neurobiological parameters correlated with hippocampal malondialdehyde content. There were significant correlations between this measure and scores of anxiety-like behaviors, as well as open field exploratory and stereotypic behaviors, and parameters of aggression. Malondialdehyde levels significantly correlated with the expression of GluA2 subunit and internalization of GluA1 and GluA2 subunits of AMPA receptor, gene expression of IL-16, TNF, GSK3B, and the number of Ki67positive cells. Besides, positive correlations were found between the number of attacks and most of investigated markers of cellular plasticity and neuroinflammation, as well as MDA concentration in the hippocampus. They are likely to be due to the independent effects of the ultrasound stress on both aggression and stress-related markers, rather than the effect of a single experience of aggressive interaction alone. Recent studies revealed a decreased number of Ki67-positive cells in the hippocampus of a mouse strain that displays a reduction of aggressive behavior after ultrasound exposure (Gorlova, personal communication). Notably, analysis of non-stressed groups alone did not reveal any significant correlations between MDA levels and above-mentioned parameters (Suppl. Fig.7). These results further suggest the importance of pro-oxidative and pro- 
inflammatory changes as mechanisms of the negative effects of ultrasound stress in mice, and the role of the hippocampus in these changes.

Chronic treatment of mice with applied herbal composition precluded ultrasound-induced increases of manifestations of anxiety-like and aggressive behaviours, as well as most of the reported molecular changes pointing to the anti-oxidant and antiinflammatory effects of the composition. Specifically, administration of herbal composition has prevented the increase in malondialdehyde content and over-expression of proinflammatory cytokines in ultrasound-exposed mice. This was accompanied by normalization of evaluated behavioural correlates of emotionality-like states, AMPA receptor subunits and expression of a marker of distress GSK3B and plasticity, Ki67, in the hippocampus. Application of herbal composition in non-stressed mice and the use of its solvent in stressed animals did not alter the majority of investigated parameters.

Previous studies demonstrated ameliorative effects of thiamine compounds with anti-oxidant and anti-inflammatory properties on the parameters of emotionality-like states and plasticity during stress (Markova et al., 2016, Vignisse et al., 2017; Pavlov et al., 2019). Other studies revealed anxiolytic-like effects of compounds with antioxidant properties (Bystritsky et al., 2012; Dourado and Cezar, 2015; Salim et al., 2017). In addition, a relationship between the anti-anxiety action and normalization of malondialdehyde content in the brain was 
demonstrated in mice (Sulakhiya et al., 2016). Thus, the beneficial functional effects of the herbal composition used in this study can be explained by its antioxidant and anti-inflammatory properties.

Treatments with the components of the herbal composition used in this study were previously shown to exert similar neurobiological effects: e.g., polysaccharides from red seaweed suppressed expression of TNF receptor-associated factor-6, protecting against LPS-induced toxicity (Cui et al., 2019); domestic brown seaweeds reduced expression of proinflammatory cytokines in activated macrophages (Kwon et al., 2013); ginger phenolics diminished lipid peroxidation and oxidative stress neuronal damage in rats (Vipin et al., 2017); vanillin suppressed free radical production in a mouse model of cancer (Tai et al., 2011). Flavonoids from citrus fruits were shown to enhance activity of anti-oxidant defense enzymes, such as superoxide dismutase, catalase and glutathione (Zaidun et al., 2018), prevent stress-induced neurotoxicity, oxidative stress (Heo et al., 2008), and over-production of inflammatory factors, including C-reactive protein, TNF, and IL-1B (Dourado and Cesar, 2015). Elderberry extract normalized the activities of superoxide dismutase and glutathione peroxidase (Salvador et al., 2016), and reduced serum IL-6 levels (Youdium et al., 2000). Finally, glutamine, a precursor of glutathione, was shown to regulate cellular redox balance (Matés et al., 2002) and prevent changes in 
gene expression associated with oxidative stress in rats (Tsai et al., 2012).

The administration of its solvent resulted in partial amelioration of several measures of behavior, IL-6 and GluA2 expression and GluA3 internalization that was reminiscent of the effects of HAT itself and is likely due to well-known anti-stress effects of alcohol (Wang et al., 2008), its main component. As chronic intake of small doses of alcohol can induce antiinflammatory effects (Stote et al., 2015; Mangnus et al., 2017) and alters AMPA receptor-mediated signaling in rodents (Ary et al. 2012; Salling et al., 2016), HAT-solvent might produce additive effects in the action of investigated here herbal cocktail.

\section{Conclusion}

Altogether, presented data emphasize the role of oxidative stress and neuroinflammation in neurobiological abnormalities associated with "emotional" stress-like state and highlight the beneficial anti-anxiety properties of the applied herbal composition. Generally, pathological changes in oxidative stress, pro-inflammatory, glutamatergic, and plasticity mechanisms during stress are inter-related, and metabolic / mitochondrial regulations can be viewed as pivotal in these dysregulatory cascades (Rezin et al., 2008; Hussain et al., 2016; Picard and McEwen, 2018). Given that patients with anxiety disorders revealed a reduction in brain markers of plasticity, signs of neuroinflammation (Michopoulos et al., 2017), and aberrant 
AMPA-receptor mediated neurotransmission (Bergink et al., 2004), it can be anticipated that the investigated herbal composition has a therapeutic potential not only for prevention of stress-related anxiety, but also under clinical conditions of elevated anxiety.

While the number of elements in the herbal composition is a limitation of this study, as the question whether any individual element or combinations of elements contribute to the effects could not be addressed, present work highlights several mechanisms related to glutamatergic neurotransmission, inflammation and oxidative stress, which are implicated in the anti-stress action of tested here herbal cocktail. Further studies are required to elucidate these mechanisms more in a detail.

\section{Acknowledgements}

The authors acknowledge the financial support from " $5-100$ " Russian Research Excellence program. RT-PCR assay from the reported study were funded by RFBR according to the research project № 18-34-00275 and herbal treatment was kindly donated by Vendeville, Voorhout, The Netherlands. We thank Ms. Diana Babaevskaya, Ms. Margarita Oplatchikova and Mr. Sergey Kurevlev for their technical contributions to this work and express special gratitude to Ms. Arina Kazakova (Mount Holyoke College, USA) for a practical help and valuable assistance in editing English language in this paper. 
Table 1. Relative gene expression of GluA1, GluA2 and GluA3 subunits

\begin{tabular}{|c|c|c|c|}
\hline \multirow{2}{*}{ Groups } & \multicolumn{3}{|c|}{ Relative-fold gene expression, \% from } \\
control \\
\cline { 2 - 4 } & GluA1 & GluA2 & GluA3 \\
\hline $\begin{array}{c}\text { Control, non- } \\
\text { treated } \\
\text { (Con NT) }\end{array}$ & $100 \pm 4.24$ & $100 \pm 5.57$ & $100 \pm 3.68$ \\
\hline $\begin{array}{c}\text { Control, HAT- } \\
\text { solvent (Con } \\
\text { HATs) }\end{array}$ & $104.7 \pm 6.17$ & $101.1 \pm 9.08$ & $111.6 \pm 6.94$ \\
\hline $\begin{array}{c}\text { Control, HAT } \\
\text { (Con HAT) }\end{array}$ & $107.9 \pm 10.67$ & $98.1 \pm 8.87$ & $111.9 \pm 7.13$ \\
\hline $\begin{array}{c}\text { US, non-treated } \\
\text { (US NT) }\end{array}$ & $67.2 \pm 7.12$ & $\begin{array}{c}135.6 \pm 10.95 \\
\text { vs Con NT }\end{array}$ & $65.9 \pm 10.85$ \\
\hline $\begin{array}{c}\text { US, HAT-solvent } \\
\text { (US HATs) }\end{array}$ & $75.4 \pm 13.81$ & $120.5 \pm 6.08$ & $78.8 \pm 7.13$ \\
\hline \multicolumn{1}{|c|}{$\begin{array}{c}\text { US, HAT } \\
\text { (US HAT) }\end{array}$} & $99.6 \pm 5.37$ & $100.7 \pm 4.97$ & $87.7 \pm 8.91$ \\
\hline \multirow{2}{*}{ (US } & & & \\
\hline
\end{tabular}

Table 1. Relative fold levels of mRNA expression of GluA1, GluA2 and GluA3 subunits normalized to control. There was a significant increase of GluA2 expression in stressed non-treated mice in comparison with control non-treated mice ( ${ }^{*} \mathrm{p}<0.05$ vs. non-treated controls, two-way ANOVA and post hoc Tukey's test. 6-8 animals per group were used, relative mRNA expression data are expressed in percent from non-treated controls). US - ultrasound, HAT - herbal antioxidant treatment. Data are Mean \pm SEM. 
Table 2. Numbers of internalized GluA1, GluA2 and GluA3 puncta

\begin{tabular}{|c|c|c|c|}
\hline \multirow{2}{*}{ Groups } & \multicolumn{3}{|c|}{ Internalized puncta, \% from to control } \\
\hline & GluA1 & GluA2 & GluA1 \\
\hline $\begin{array}{c}\text { Control, non- } \\
\text { treated } \\
\text { (Con NT) }\end{array}$ & $100 \pm 20.49$ & $100 \pm 16.56$ & $100 \pm 18.01$ \\
\hline $\begin{array}{c}\text { Control, HAT- } \\
\text { solvent (Con } \\
\text { HATs) } \\
\end{array}$ & $94.0 \pm 11.07$ & $101.2 \pm 14.41$ & $103 \pm 5.99$ \\
\hline $\begin{array}{c}\text { Control, HAT } \\
\text { (Con HAT) }\end{array}$ & $113.6 \pm 17.28$ & $89.4 \pm 10.01$ & $90 \pm 16.02$ \\
\hline $\begin{array}{l}\text { US, non-treated } \\
\text { (US NT) }\end{array}$ & $\begin{array}{c}227.8 \pm 18.40 \\
\text { vs Con NT } \\
{ }^{*} \mathrm{p}<0.001\end{array}$ & $\begin{array}{c}203.6 \pm 22.54 \\
\text { vs Con NT } \\
{ }^{*} \mathrm{p}=0.003\end{array}$ & $\begin{array}{c}163.8 \pm 15.79 \\
\text { vs Con NT } \\
{ }^{*} \mathrm{p}=0.043\end{array}$ \\
\hline $\begin{array}{l}\text { US, HAT-solvent } \\
\text { (US HATs) }\end{array}$ & $162.6 \pm 12.33$ & $\begin{array}{c}164.6 \pm 5.19 \\
\text { vs Con HATs } \\
{ }^{*} \mathrm{p}=0.040\end{array}$ & $135.2 \pm 13.42$ \\
\hline $\begin{array}{l}\text { US, HAT } \\
\text { (US HAT) }\end{array}$ & $\begin{array}{c}104.6 \pm 14.43 \\
\text { vs US NT } \\
\# \mathrm{p}<0.001\end{array}$ & $\begin{array}{l}110.8 \pm 8.03 \\
\text { vs US NT } \\
\# p=0.001\end{array}$ & $122.2 \pm 12.60$ \\
\hline
\end{tabular}

Table 2. Numbers of internalized GluA1, GluA2 and GluA3 puncta. Numbers of internalized GluA1-3 puncta in stressed non-treated mice and number of internalized GluA2 puncta in stressed mice treated with HAT-solvent were increased in comparison with control non-treated mice. HAT-treated stressed mice displayed a decrease of number of internalized GluA1-2 puncta in comparison to stressed non-treated group $\left({ }^{*} \mathrm{p}<0.05\right.$ vs. non-treated controls, $\# \mathrm{p}<0.05$ vs. non-treated stressed group, two-way ANOVA and post hoc Tukey's test. 6-8 animals per group were used, data are expressed in percent from non-treated controls). US - ultrasound, HAT - herbal antioxidant treatment. Data are Mean \pm SEM. 
Table 3. Numbers of surface GluA1, GluA2 and GluA3 puncta

\begin{tabular}{|c|c|c|c|}
\hline \multirow{2}{*}{ Groups } & \multicolumn{2}{|c|}{ Surface puncta, \% from control } \\
\cline { 2 - 4 } & GluA1 & GluA2 & GluA1 \\
\hline $\begin{array}{c}\text { Control, non- } \\
\text { treated } \\
\text { (Con NT) }\end{array}$ & $100 \pm 15.97$ & $100 \pm 14.06$ & $100 \pm 15.26$ \\
\hline $\begin{array}{c}\text { Control, HAT- } \\
\text { solvent (Con } \\
\text { HATs) }\end{array}$ & $113.6 \pm 7.29$ & $97.6 \pm 9.65$ & $99.2 \pm 7.93$ \\
\hline $\begin{array}{c}\text { Control, HAT } \\
\text { (Con HAT) }\end{array}$ & $108.6 \pm 10.47$ & $106 \pm 11.46$ & $90 \pm 16.02$ \\
\hline $\begin{array}{c}\text { US, non-treated } \\
\text { (US NT) }\end{array}$ & $46 \pm 6.47$ & $65 \pm 18.9$ & $61.4 \pm 4.76$ \\
\hline $\begin{array}{c}\text { US, HAT-solvent } \\
\text { (US HATs) }\end{array}$ & $58.2 \pm 9.59$ & $61.2 \pm 7.69$ & $53.6 \pm 7.95$ \\
\hline $\begin{array}{c}\text { US, HAT } \\
\text { (US HAT) }\end{array}$ & $79.2 \pm 13.85$ & $\begin{array}{l}112.4 \pm 9.82 \\
\text { vs US NT } \\
\# p=0.016\end{array}$ & $\begin{array}{c}120.6 \pm 14.6 \\
\text { vs US NT } \\
\# p=0.008\end{array}$ \\
\hline
\end{tabular}

Table 3. Numbers of surface GluA1, GluA2 and GluA3 puncta. Number of surface GluA2-3 puncta were significantly increased in stressed HAT-treated mice in comparison with stressed non-treated mice $(\# \mathrm{p}<0.05$ vs. non-treated stressed group, two-way ANOVA and post hoc Tukey's test. 6-8 animals per group were used, data are expressed in percent from nontreated controls). US - ultrasound, HAT - herbal antioxidant treatment. Data are Mean \pm SEM. 
Table 4. Internalization index of GluA1, GluA2 and GluA3 subunits

\begin{tabular}{|c|c|c|c|}
\hline \multirow{2}{*}{ Groups } & \multicolumn{3}{|c|}{ Internalization index, \% } \\
\hline & GluA1 & GluA2 & GluA1 \\
\hline $\begin{array}{c}\text { Control, non- } \\
\text { treated } \\
\text { (Con NT) }\end{array}$ & $49.2 \pm 6.1$ & $49.5 \pm 5.18$ & $49.6 \pm 6.83$ \\
\hline $\begin{array}{c}\text { Control, HAT- } \\
\text { solvent (Con HATs) }\end{array}$ & $44.9 \pm 2.19$ & $50.4 \pm 2.69$ & $51.1 \pm 1.17$ \\
\hline $\begin{array}{l}\text { Control, HAT } \\
\text { (Con HAT) }\end{array}$ & $51.9 \pm 7.47$ & $45.8 \pm 3.97$ & $46.4 \pm 4.46$ \\
\hline $\begin{array}{l}\text { US, non-treated (US } \\
\text { NT) }\end{array}$ & $\begin{array}{c}82.9 \pm 2.71 \\
\text { vs Con } \\
\text { NT } \\
{ }^{*} \mathrm{p}<0.0001\end{array}$ & $\begin{array}{c}75.1 \pm 4.06 \\
\text { vs Con NT } \\
{ }^{*} \mathrm{p}=0.003\end{array}$ & $\begin{array}{c}72.1 \pm 3.36 \\
\text { vs Con NT } \\
\text { *p }=0.0419\end{array}$ \\
\hline $\begin{array}{l}\text { US, HAT-solvent } \\
\text { (US HATs) }\end{array}$ & $\begin{array}{c}73.8 \pm 4.17 \\
\text { vs Con } \\
\text { NT } \\
{ }^{*} \mathrm{p}<0.0001\end{array}$ & $\begin{array}{c}73.2 \pm 2.09 \\
\text { vs Con NT } \\
* \mathrm{p}=0.0002\end{array}$ & $\begin{array}{c}71.1 \pm 4.88 \\
\text { vs Con NT } \\
{ }^{*} \mathrm{p}=0.157\end{array}$ \\
\hline $\begin{array}{l}\text { US, HAT } \\
\text { (US HAT) }\end{array}$ & $\begin{array}{l}56.7 \pm 7.19 \\
\text { vs US NT } \\
\# \mathrm{p}=0.0001\end{array}$ & $\begin{array}{c}49.7 \pm 3.73 \\
\text { vs US NT } \\
\# p<0.0001\end{array}$ & $\begin{array}{l}50.4 \pm 4.61 \\
\text { vs US NT } \\
\# p=0.012\end{array}$ \\
\hline
\end{tabular}

Table 4. Internalization index of GluA1, GluA2 and GluA3 subunits. Internalization index of GluA1-3 subunits in stressed non-treated mice and stressed mice treated with HATsolvent was significantly increased in comparison with nontreated controls. This parameter was significantly decreased in stressed HAT-treated mice in comparison to stressed non-treated group in all subunits (*p<0.05 vs. non-treated controls, $\# p<0.05$ vs. non-treated stressed group, two-way ANOVA and post hoc Tukey's test. 6-8 animals per group were used). US - ultrasound, HAT - herbal antioxidant treatment. Data are Mean \pm SEM. 


\section{Reference list}

Adamczyk A., Mejias R., Takamiya K., Yocum J., Krasnova I.N., Calderon J., Cadet J.L., Huganir R.L., Pletnikov M.V., Wang T. GluA3-deficiency in mice is associated with increased social and aggressive behavior and elevated dopamine in striatum. Behav Brain Res. 2012; 229: 265-272.

Anderson G.D., Hakimian S. Pharmacokinetic Factors to Consider in the Selection of Antiseizure Drugs for Older Patients with Epilepsy. Drugs Aging. 2018; 35: 687-698.

Ary A.W., Cozzoli D.K., Finn D.A., Crabbe J.C., Dehoff M.H., Worley P.F., Szumlinski K.K. Ethanol up-regulates nucleus accumbens neuronal activity dependent pentraxin (Narp): implications for alcohol-induced behavioral plasticity. Alcohol. 2012; 46: 377-387.

Ayala A., Muñoz M.F., Argüelles S. Lipid Peroxidation: Production, Metabolism, and Signaling Mechanisms of Malondialdehyde and 4-Hydroxy-2-Nonenal. Oxid Med Cell Longev. 2014: 360438.

Bandelow B., Michaelis S. Epidemiology of anxiety disorders in the 21st century. Dialogues Clin Neurosci. 2005; 17: 327-335.

Bergink V., van Megen H.J., Westenberg H.G. Glutamate and anxiety. Eur Neuropsychopharmacol. 2004; 14: 175-183.

Beurel E., Grieco S.F., Jopea R.S. Glycogen synthase kinase-3 (GSK3): regulation, actions, and diseases. Pharmacol Ther. 2015,114131.

Bonini D., Mora C., Tornese P., Sala N., Filippini A., La Via L., Milanese M., Calza S., Bonanno G., Racagni G., Gennarelli M., Popoli M., Musazzi L., Barbon A. Acute Footshock Stress Induces TimeDependent Modifications of AMPA/NMDA Protein Expression and AMPA Phosphorylation. Neural Plast. 2016: 7267865.

Bouayed J., Rammal H., Dicko A., Younos C., Soulimani R. Chlorogenic acid, a polyphenol from Prunus domestica (Mirabelle), with coupled anxiolytic and antioxidant effects. J Neurol Sci. 2007; 262: 77-84.

Bowers M.S., Chen B.T., Bonci A. AMPA receptor synaptic plasticity induced by psychostimulants: the past, present, and therapeutic future. Neuron. 2010; 67: 11-24.

Bystritsky A., Hovav S., Sherbourne C., Stein M.B., Rose R.D, Campbell-Sills L., Golinelli D., Sullivan G., Craske M.G., RoyByrne P.P. Use of complementary and alternative medicine in a large sample of anxiety patients. Psychosomatics. 2012; 53: 266272 . 
Campbell S., MacQueen G. The role of the hippocampus in the pathophysiology of major depression. J Psychiatry Neurosci. 2004; 29: 417-426.

Caudal D., Godsil B.P., Mailliet F., Bergerot D., Jay T.M. Acute Stress Induces Contrasting Changes in AMPA Receptor Subunit Phosphorylation within the Prefrontal Cortex, Amygdala and Hippocampus. PLoS One. 2010; 5: e15282.

Chen J, Song Y, Yang J, Zhang Y, Zhao P, Zhu XJ, Su HC. The contribution of TNF- $\alpha$ in the amygdala to anxiety in mice with persistent inflammatory pain. Neurosci Lett. 2013; 541: 275-280.

Cline B.H., Anthony D.C., Lysko A., Dolgov O., Anokhin K., Schroeter C., Malin D., Kubatiev A., Steinbusch H.W., Lesch K.P., Strekalova T. Lasting downregulation of the lipid peroxidation enzymes in the prefrontal cortex of mice susceptible to stressinduced anhedonia. Behav Brain Res. 2015; 276: 118-129.

Cline B.H., Steinbusch H.W., Malin D., Revishchin A.V., Pavlova G.V., Cespuglio R., Strekalova T. The neuronal insulin sensitizer dicholine succinate reduces stress-induced depressive traits and memory deficit: possible role of insulin-like growth factor 2 . BMC Neurosci. 2012; 13:110.

Costa-Nunes J., Zubareva O., Araújo-Correia M., Valença A., Schroeter C.A., Pawluski J.L., Vignisse J., Steinbusch H., Hermes D., Phillipines M., Steinbusch H.M., Strekalova T. Altered emotionality, hippocampus-dependent performance and expression of NMDA receptor subunit mRNAs in chronically stressed mice.Stress. 2014; 17: 108-116.

Couch Y., Anthony D.C., Dolgov O., Revischin A., Festoff B., Santos A.I., Steinbusch H.W., Strekalova T. Microglial activation, increased TNF and SERT expression in the prefrontal cortex define stressaltered behaviour in mice susceptible to anhedonia. Brain Behav. Immun. 2013; 29: 136-146.

Couch Y., Trofimov A., Markova N., Nikolenko V., Steinbusch H.W., Chekhonin V., Schroeter C., Lesch K.P. Low-dose lipopolysaccharide (LPS) inhibits aggressive and augments depressive behaviours in a chronic mild stress model in mice. $\mathrm{J}$ Neuroinflammation. 2016; 13: 1-8.

Cui M., Wu J., Wang S., Shu H., Zhang M., Liu K., Liu K.. Characterization and anti-inflammatory effects of sulfated polysaccharide from the red seaweed Gelidium pacificum Okamura. Int J Biol Macromol. 2019; 129: 377-385.

Dourado G.K.Z.S., Cesar T.B. Investigation of cytokines, oxidative stress, metabolic, and inflammatory biomarkers after orange 
juice consumption by normal and overweight subjects. Food Nutr Res. 2015; 59: 10.3402/fnr.v59.28147.

Fontes M.A., Xavier C.H., Marins F.R., Limborço-Filho M., Vaz G.C., Müller-Ribeiro F.C., Nalivaiko E. Emotional stress and sympathetic activity: Contribution of dorsomedial hypothalamus to cardiac arrhythmias.Brain Research. 2014; 1554: 49-58.

Gorlova A., Pavlov D., Anthony D.C., Ponomarev E., Proshin A., Sambon M., Schmitt-Boeher A., Lesch K.P., Bettendorff L., Strekalova T. Thiamine and benfotiamine counteract ultrasound-induced aggression, normalize AMPA receptor expression and plasticity markers, and reduce oxidative stress in mice. Neuropharmacology, 2019, 156: 107543

Green H.F., Nolan Y.M. GSK-3 mediates the release of IL-18, TNF- $\alpha$ and IL-10 from cortical glia. Neurochem Int. 2012; 61: 666-671.

Grenier S., Desjardins F., Raymond B., Payette M.C., Rioux M.È., Landreville P., Gosselin P., Richer M.J., Gunther B., Fournel M., Vasiliadis H.M. Six-month prevalence and correlates of generalized anxiety disorder among primary care patients aged 70 years and above: Results from the ESA-services study. Int $\mathrm{J}$ Geriatr Psychiatry. 2019; 34: 315-323.

Heo H.J., Choi S.J., Choi S.G., Shin D.H., Lee J.M., Lee C.Y. Effects of banana, orange, and apple on oxidative stress-induced neurotoxicity in PC12 cells. J Food Sci. 2008; 73: 28-32.

Hussain T., Tan B., Yin Y., Blachier F., Tossou M.C., Rahu N. Oxidative Stress and Inflammation: What Polyphenols Can Do for Us? Oxid Med Cell Longev. 2016: 7432797.

Kalueff A.V., Keisala T., Minasyan A., Kuuslahti M., Tuohimaa P.. Temporal stability of novelty exploration in mice exposed to different open field tests. Behav Processes. 2006; 72: 104-112.

Kessler R.C., Petukhova M., Sampson N.A., Zaslavsky A.M., Wittchen H.U. Twelve-month and lifetime prevalence and lifetime morbid risk of anxiety and mood disorders in the United States. Int J Methods Psychiatr Res. 2012; 21: 169-184.

Kwon J., Kim J., Park S., Khang G., Kang P.M., Lee D. Inflammationresponsive antioxidant nanoparticles based on a polymeric prodrug of vanillin. Biomacromolecules. 2013; 14: 1618-1626.

Lajud N., Roque A., Cajero M., Gutiérrez-Ospina G., Torner L. Periodic maternal separation decreases hippocampal neurogenesis without affecting basal corticosterone during the stress hyporesponsive period, but alters HPA axis and coping behavior in adulthood. Psychoneuroendocrinology. 2012; 37: 410-420. 
Mangnus L., van Steenbergen H.W., Nieuwenhuis W.P., Reijnierse M., van der Helm-van Mil A.H.M. Moderate use of alcohol is associated with lower levels of $\mathrm{C}$ reactive protein but not with less severe joint inflammation: a cross-sectional study in early RA and healthy volunteers. RMD Open. 2018; 4: e000577.

Manning B.D., Toker A. AKT/PKB Signaling: Navigating the Network. Cell. 2017; 169: 381-405.

Martinowich K., Schloesser $\quad$ R.J., Lu Y., Jimenez D.V., Paredes D., Greene J.S., Greig N.H., Manji H.K., Lu B. Roles of p75(NTR), long-term depression, and cholinergic transmission in anxiety and acute stress coping. Biol Psychiatry. 2012; 71: 7583.

Markova N., Bazhenova N., Anthony D.C., Vignisse J., Svistunov A., Lesch K.P., Bettendorff L., Strekalova T.. Thiamine and benfotiamine improve cognition and ameliorate GSK-3Bassociated sterss-induced behaviours in mice. Prog NeuroPsychopharmacol Biol Psychiatry. 2016; 75: 148-156.

Matés J.M., Pérez-Gómez C., Núñez de Castro I., Asenjo M., Márquez J. Glutamine and its relationship with intracellular redox status, oxidative stress and cell proliferation/death. Int $\mathrm{J}$ Biochem Cell Biol. 2002; 34: 439-458.

McIntyre E., Saliba A.J., Moran C.C. Herbal medicine use in adults who experience anxiety: A qualitative exploration. Int J Qual Stud Health Well-being. 2015; 10: 10.3402/qhw.v10.29275.

Michopoulos V., Powers A., Gillespie C.F., Ressler K.J., Jovanovic T. Inflammation in Fear- and Anxiety-Based Disorders: PTSD, GAD, and Beyond. Neuropsychopharmacology. 2017; 42: 254270 .

Morozova A., Zubkov E., Strekalova T., Kekelidze Z., Storozeva Z., Schroeter C.A., Bazhenova N., Lesch K.P., Cline B.H., Chekhonin V. Ultrasound of alternating frequencies and variable emotional impact evokes depressive syndrome in mice and rats. Prog Neuro-Psychopharmacol Biol Psychiatry. 2016; 68: 52-63.

Orsolini L., Bellantuono C. Serotonin reuptake inhibitors and breastfeeding: a systematic review. Hum. Psychopharmacol Clin Exp. 2015; 30: 4-20.

Oyebode O., Kandala N., Chilton P., Lilford R. Use of traditional medicine in middle-income countries: a WHO-SAGE study. Health Policy Plan. 2016; 31: 984-991.

Pavlov D., Bettendorff L., Gorlova A., Olkhovik A., Kalueff A.V., Ponomarev E.D., Inozemtsev A., Chekhonin V., Lesch K.P., 
Anthony D.C., Strekalova T.. Neuroinflammation and aberrant hippocampal plasticity in a mouse model of emotional stress evoked by exposure to ultrasound of alternating frequencies. Prog Neuropsychopharmacol Biol Psychiatry. 2016; 90: 104-116. Peineau S., Bradley C., Taghibiglou C., Doherty A., Bortolotto Z.A., Wang Y.T., Collingridge G.L. The role of GSK-3 in synaptic plasticity. Br J Pharmacol. 2008; 153: S428-S437.

Picard M., McEwen B.S. Psychological Stress and Mitochondria: A Systematic Review. Psychosom Med.2018; 80:141-153.

Potz B.A., Scrimgeour L.A., Sabe S.A., Clements R.T., Sodha N.R., Sellke F.W. Glycogen Synthase Kinase 36 Inhibition Reduces Mitochondrial Oxidative Stress in Chronic Myocardial Ischemia. J Thorac Cardiovasc Surg. 2018; 155: 2492-2503.

Pradhan A.A., Tawfik V.L., Laboy A.F., Scherrer G. In Vivo Techniques to Investigate the Internalization Profile of Opioid Receptors. Methods Mol Biol. 2016; 1230: 187-194.

Quagliato L.A., Cosci F., Shader R.I., Silberman E.K., Starcevic V., Balon R., Dubovsky S.L., Salzman C., Krystal J.H., Weintraub S.J., Freire R.C., Nardi A.E. Selective serotonin reuptake inhibitors and benzodiazepines in panic disorder: A metaanalysis of common side effects in acute treatment. Journal of Psychopharmacology. 2019. 1-12.

Rezin G.T., Cardoso M.R., Gonçalves C. L., Scaini G., Fraga D.B., Riegel R.E., Comim C.M., Quevedo J., Streck E.L. Inhibition of mitochondrial respiratory chain in brain of rats subjected to an experimental model of depression. Neurochemistry International. 2008; 53: 395-400.

Rossi S., Studer V., Motta C., Polidoro S., Perugini J., Macchiarulo G., Giovannetti A.M., Pareja-Gutierrez L., Calò A., Colonna I., Furlan R., Martino G., Centonze D. Neuroinflammation drives anxiety and depression in relapsing-remitting multiple sclerosis. Neurology. 2017; 89: 1338-1347.

Salim S. Oxidative Stress and the Central Nervous System. J Pharmacol Exp Ther. 2017; 360: 201-205.

Salling M.C., Faccidomo S.P., Li C., Psilos K., Galunas C., Spanos M., Agoglia A.E., Kash T.L., Hodge C.W. Moderate Alcohol Drinking and the Amygdala Proteome: Identification and Validation of Calcium/Calmodulin Dependent Kinase II and AMPA Receptor Activity as Novel Molecular Mechanisms of the Positive Reinforcing Effects of Alcohol. Biol Psychiatry. 2016; 79: 430442 . 
Salvador A.C., Król E., Lemos V.C., Santos S.A.O., Bento F.P.M.S., Costa C.P. Almeida A., Szczepankiewicz D., Kulczyński B., Krejpcio Z., Silvestre A.J.D., Rocha1 S.M. Effect of Elderberry (Sambucus nigra L.) Extract Supplementation in STZ-Induced Diabetic Rats Fed with a High-Fat Diet. Int J Mol Sci. 2016; 18: 13.

Schiavone S., Colaianna M., Curtis L. Impact of early life stress on the pathogenesis of mental disorders: relation to brain oxidative stress. Curr Pharm Des. 2015; 21: 1404-1412.

Schoenfeld T., Gould E. Stress, Stress Hormones, and Adult Neurogenesis. Exp Neurol. 2012; 233(1): 12-21.

Stone M.H. Long-Term Course of Borderline Personality Disorder. Psychodyn. Psychiatry. 2016; 244: 449-474.

Stote K.S., Tracy R.P., Taylor P.R., Baer D.J. The effect of moderate alcohol consumption on biomarkers of inflammation and hemostatic factors in postmenopausal women. Eur J Clin Nutr. 2016; 70: 470-474.

Strekalova T., Bahzenova N., Trofimov A., Schmitt-Böhrer A.G., Markova N., Grigoriev V., Zamoyski V., Serkova T., Redkozubova O., Vinogradova D., Umriukhin A., Fisenko V., Lillesaar C., Shevtsova E., Sokolov V., Aksinenko A., Lesch k.P., Bachurin S. Pro-neurogenic, Memory-Enhancing and Antistress Effects of DF302, a Novel Fluorine Gamma-Carboline Derivative with Multi-target Mechanism of Action. Mol Neurobiol. 2018; 55: 335-349.

Strekalova T., Markova N., Shevtsova E., Zubareva O., Bakhmet A., Steinbusch H.M., Bachurin S., Lesch K.P. Individual Differences in Behavioural Despair Predict Brain GSK-3beta Expression in Mice: The Power of a Modified Swim Test. Neural Plast. 2016:5098591.

Strekalova T., Spanagel R., Bartsch D., Henn F.A., Gass P.Stressinduced anhedonia in mice is associated with deficits in forced swimming and exploration. Neuropsychopharmacology. 2004; 29: 2007-2017.

Strekalova T., Spanagel R., Dolgov O., Bartsch D. Stress-induced hyperlocomotion as a confounding factor in anxiety and depression models in mice. Behav Pharmacol. 2005; 16: 171-80.

Sulakhiya K., Patel V.K., Saxena R., Dashore J., Srivastava A.K., Rathore M. Effect of Beta vulgaris Linn. Leaves Extract on Anxiety- and Depressive-like Behavior and Oxidative Stress in Mice after Acute Restraint Stress. Pharmacognosy Res. 2016; 8: 1-7. 
Tai A., Sawano T., Yazama F., Ito H. Evaluation of antioxidant activity of vanillin by using multiple antioxidant assays. Biochim Biophys Acta. 2011; 1810: 170-177.

Takahashi A., Flanigan M.E., McEwen B.S., Russo S.J. Aggression, Social Stress, and the Immune System in Humans and Animal Models. Front Behav Neurosci. 2018; 12: 56.

Tsai P.H., Liu J.J., Yeh C.L., Chiu W.C., Yeh S.L. Effects of glutamine supplementation on oxidative stress-related gene expression and antioxidant properties in rats with streptozotocin-induced type 2 diabetes. Br J Nutr. 2012; 107: 1112-1118.

Umukoro S., Adebesin A., Agu G., Omorogbe O., Asehinde S.B. Antidepressant-like activity of methyl jasmonate involves modulation of monoaminergic pathways in mice. Adv Med Sci. 2018; 63: 36-42.

Uzun S., Kozumplik O., Jakovljević M., Sedić B. Side effects of treatment with benzodiazepines. Psychiatr Danub. 2010; 22: 9093.

van Rijnsoever C., Sidler C., Fritschy J.M. Internalized GABA-receptor subunits are transferred to an intracellular pool associated with the postsynaptic density. Eur J Neurosci. 2005; 21: 327-338.

Vignisse J., Sambon M., Gorlova A., Pavlov D., Caron N., Malgrange B., Shevtsova E.,Svistunov A., Anthony D., Markova N., Bazhenova N., Coumans B., Lakaye B., Wins P., Strekalova T., Bettendorff L. Thiamine and benfotiamine prevent stress-induced suppression of hippocampal neurogenesis in mice exposed to predation without affecting brain thiamine diphosphate levels. Molecular and Cellular Neuroscience. 2017; 82: 126-136.

Violle N., Rozan P., Demais H., Nyvall Collen P., Bisson J.F.. Evaluation of the antidepressant- and anxiolytic-like effects of a hydrophilic extract from the green seaweed Ulva sp. in rats. 2018; 21: 248-256.

Vipin A.V., Rao R., Kurrey N.K., Anu A.K.A., Venkateswaran G.. Protective effects of phenolics rich extract of ginger against Aflatoxin B1-induced oxidative stress and hepatotoxicity. Biomed Pharmacother. 2017; 91: 415-424.

Wang J.J., Tung T.H., Yin W.H., Huang C.M., Jen H.L., Wei J., Young M.S. Effects of moderate alcohol consumption on inflammatory biomarkers. Acta Cardiol. 2008; 63: 65-72.

Weber T., Vogt M.A., Gartside S.E., Berger S.M., Lujan R., Lau T., Herrmann E., Sprengel R., Bartsch D., Gass P. Adult AMPA GLUA1 receptor subunit loss in 5-HT neurons results in a specific anxiety-phenotype with evidence for dysregulation of 5- 
HT neuronal activity. Neuropsychopharmacology. 2015; 40: 1471-1484.

Youdim K.A., Martin A., Joseph J.A. Incorporation of the elderberry anthocyanins by endothelial cells increases protection against oxidative stress. Free Radic Biol Med. 2000; 29: 51-60.

Zaidun N.H., Thent Z.C., Latiff A.A. Combating oxidative stress disorders with citrus flavonoid: Naringenin. Life Sci. 2018; 208: 111-122.

\section{Supplementary material}

\section{Ultrasound emission}

The selectivity of the adverse effects of low-frequency ultrasound during the emission period in comparison with the potential general negative effects of a constant noise described here was demonstrated previously (Morozova et al., 2016; Gorlova et al., 2019). The shape of the ultrasound signals was fluctuating, mimicking natural ultrasonic vocalizations of rodents (Fig.1).

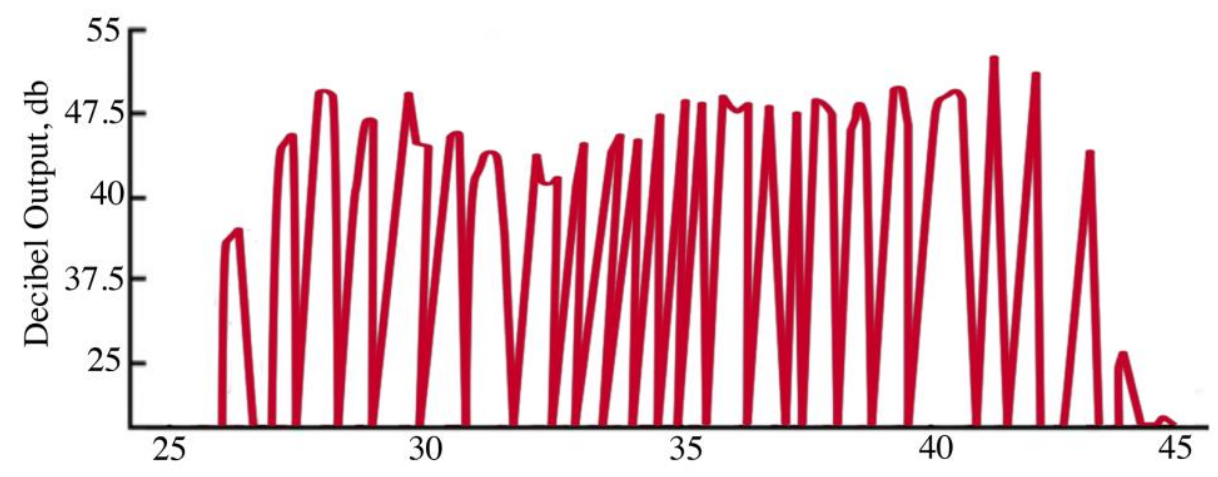

Frequency, $\mathrm{kHz}$

Supplementary Figure 1. Representation of the ultrasound signal mimicking a natural sound of mice: frequency-intensity fluctuations. 
Composition of herbal treatment and its solvent

The drops are made out of herbs, spices and/or dried fruit diluted in 30\% alcohol solvent Vendeville, Voorhout, The Netherlands). The herbs and spices are free of toxins.

Supplementary Table 1. Composition of herbal treatment (HAT) comprising of fractions A-D and S-D and HAT-solvent

\begin{tabular}{|c|c|c|}
\hline HAT fraction S-A & HAT fraction S-D & HAT-solvent \\
\hline $\begin{array}{c}\text { HAT-solvent }-\mathbf{2 0 g} \\
\text { Sea-salt }-\mathbf{0 . 5 g} \\
\text { Ginger }-\mathbf{1 g} \\
\text { Seaweed }-\mathbf{0 . 2 g} \\
\text { Vanilla } \mathbf{0 . 0 1 g}\end{array}$ & $\begin{array}{c}\text { HAT-solvent }-\mathbf{3 0 g} \\
\text { Ginger }-\mathbf{1 g} \\
\text { Dried lemon }-\mathbf{0 . 1} \mathbf{g} \\
\text { Dried orange }-\mathbf{0 . 3} \mathbf{g} \\
\text { Dried elder berries }-\mathbf{0 . 2 g} \\
\text { Glutamine }-\mathbf{0 . 4 g}\end{array}$ & $\begin{array}{l}30 \% \text { alcohol produced } \\
\text { from corn, based on } \\
\text { purified distillate } \\
\text { water, gluten-free }\end{array}$ \\
\hline
\end{tabular}

Supplementary Table 2. A comparison of doses of components used in HAT-treatment against doses used in animal and human studies

\begin{tabular}{|c|c|c|c|}
\hline \multirow{2}{*}{ Ingredients } & \multicolumn{3}{|c|}{ Doses } \\
\cline { 2 - 4 } & $\begin{array}{c}\text { HAT, } \\
\mathbf{m g} / \mathbf{k g} / \mathbf{d a y}\end{array}$ & $\begin{array}{c}\text { Animal } \\
\text { studies, } \\
\mathbf{m g} / \mathbf{k g} / \mathbf{d a y}\end{array}$ & $\begin{array}{c}\text { Human } \\
\text { studies, } \\
\mathbf{m g} / \mathbf{k g}\end{array}$ \\
\hline Ginger & 160 & $200-500$ & $1.43-28.6$ \\
\hline Orange & 32 & $10-50$ & 12.9 \\
\hline Lemon & 11 & $5-20$ & 28.6 \\
\hline Elderberries & 22 & $200-1200$ & $3.58-12.9$ \\
\hline Seaweed & 10 & $3-27$ & $7.15-28.6$ \\
\hline Glutamine & 45 & 20 & 0.7 \\
\hline
\end{tabular}


Indeed, the main herbal elements of HAT used here were shown to be efficacious in comparable ranges of doses in previous studies with small rodents and in humans. For example, in the study investigating analgesic and antipyretic properties of ginger, the oral dose ginger administrated orally for 4 weeks, was 200 $\mathrm{mg} / \mathrm{kg} /$ day (Badawi et al., 2019). Another study on mice employed daily oral dosing with ginger at concentrations 250 or 500 $\mathrm{mg} / \mathrm{kg} /$ day for 20 days. In this work, no abnormalities in behavior, growth, or food and water consumption were found in gingertreated animals (El Mazoudy \& Attia, 2018).

In another mouse study using extracts from Citrus peel, orange peel oil was administered at the dose of $10 \mathrm{mg} / \mathrm{kg} / \mathrm{day}$ for 22 days (Ajikumaran et al., 2017). The antidiarrheal activity of hexane extract of Citrus lemon peel was investigated on rats that received this treatment orally at the doses of 5, 10 and 20 $\mathrm{mg} / \mathrm{kg} /$ day (Nair et al., 2017). In the study investigating antiinflammatory effects of orange during irritable bowel syndrome in mice, the orange liqueur was used at the dose of $50 \mathrm{mg} / \mathrm{kg} / \mathrm{day}$ for 7 days (Pacheco et al., 2018). For experiments addressing anticonvulsant activities of methanolic extracts of fruit and leaf of elderberries, doses of 250,500 and $1000 \mathrm{mg} / \mathrm{kg} /$ day were used in mice (Ataee et al., 2016). Elements of elderberries were administrated in a form of an extract at doses of 200-1200 $\mathrm{mg} / \mathrm{kg} /$ day to mice in order to study the potential antidepressant activities of this treatment (Mahmoudi et al., 2014). In the mouse study, anti-inflammatory effects of the red seaweed Gracilaria 
cornea were shown at the doses of 3,9 or $27 \mathrm{mg} / \mathrm{kg} /$ day (Coura et al., 2012). The administration of brown seaweed Sargassum wightii and seagrass Halophila ovalis to rats using doses of 5 or $10 \mathrm{mg} / \mathrm{kg} /$ day evoked an antinociceptive and anti-inflammatory effects (Neelakandan and Venkatesan, 2016). In rats, glutamine supplementation at the dose of $20 \mathrm{mg} / \mathrm{kg} /$ day has increased protein synthesis rate in several tissues (Boza et al., 2001).

Studies in humans report a broad range of concentrations of herbal elements to produce therapeutic effects. Human volunteers were treated with ginger at doses ranging from $100 \mathrm{mg}$ to $2 \mathrm{~g}$ per day and were tested of markers inflammation and swelling (Zick et al., 2008). A 3-week administration of the ginger extract at the dose of $1 \mathrm{~g}$ per day in volunteers was found to be effective against nausea and vomiting (Thomson et al., 2014). Extract of bitter orange at the dose of $900 \mathrm{mg} /$ day was shown to induce anti-inflammatory effects (Stohs et al., 2012). Positive effects on pancreatic functions were reported in patients that received $2 \mathrm{ml} / \mathrm{kg}$ / day of lemon/orange juice (Chu et al., 2010). The elderberry extract administered to patients with respiratory bacterial infections at doses of $600-900 \mathrm{mg} /$ day was reported to have antimicrobial activity against human pathogens (Tiralongo et al., 2016). Similar effects were demonstrated in volunteers that were acutely treated with $30 \mathrm{ml}-200 \mathrm{ml}$ of elderberry extract (Frank et al., 2007). In the study that addressed the impact of a polyphenol-rich seaweed extract on postprandial glycaemia in healthy adults, participants that consumed $500 \mathrm{mg}$ of $2000 \mathrm{mg}$ of 
the extract experienced positive effects of the dosing (Murray et al., 2018). Doses of $50 \mathrm{mg} / \mathrm{kg}$ of glutamine were found to be beneficial in humans inducing the anti-fatigue effect (Gleeson, 2008).

In summary, as for the main herbs used for HAT, available literature reports the efficacy of doses of herbal components of HAT in rodents that are comparable to concentration ranges applied in the present work. As for studies in humans, these doses are lower as expected (see Suppl. Table 2). For ginger, it is 200$500 \mathrm{mg} / \mathrm{kg} /$ day in rodents (160 in HAT) and in humans 100-2000 $\mathrm{mg} /$ day (i.e., $1.43-28.6 \mathrm{mg} / \mathrm{kg}$ ); for orange, $10-50 \mathrm{mg} / \mathrm{kg} /$ day in rodents (32 in HAT) and in humans $900 \mathrm{mg} /$ day (i.e., $12.9 \mathrm{mg} / \mathrm{kg}$ ); for lemon, $5-20 \mathrm{mg} / \mathrm{kg} /$ day in rodents (11 in HAT) $2 \mathrm{ml} / \mathrm{kg}$ in human studies $(28.6 \mathrm{mg} / \mathrm{kg})$; for elderberries, $200-1200 \mathrm{mg} / \mathrm{kg}$ in rodents (22 in HAT) and 600-900 $\mathrm{mg}$ in humans (i.e., 3.58-12.9 $\mathrm{mg} / \mathrm{kg}$ ); for seaweed - $3-27 \mathrm{mg} / \mathrm{kg} /$ day in rodents (10 in HAT) and 500-2000 mg/ day (i.e., 7.15-28.6 mg/ kg) in humans; for vanilla $100-150 \mathrm{mg} / \mathrm{kg} /$ day in rodents (1.6 in HAT) and $1000 \mathrm{mg} /$ day (i.e., $14.3 \mathrm{mg} / \mathrm{kg}$ ) in humans; for glutamine $-20 \mathrm{mg} / \mathrm{kg} / \mathrm{day}$ in rodents (45 in HAT) and $50 \mathrm{mg}$ (i.e., $0.7 \mathrm{mg} / \mathrm{kg}$ ) in humans. A difference in effective doses between animal and human studies is due to a about 50-100-fold difference in metabolic rate between mice and humans, a well-established factor with pre-clinical investigation of therapeutically active compounds and pharmaca.

Thus, the main elements of HAT used here in aboveindicated doses, were reported to be efficacious in comparable 
ranges of doses in previous studies with small rodents. Lower doses of herbal components of HAT were employed and reported to be efficacious in human studies; the difference in the doses used between rodent and human studies is due to distinct metabolic rates in two species that is 50-100 fold slower in the latter.

Quantitative reverse transcription polymerase chain reaction analysis (qRT-PCR)

qRT-PCR was performed using CFX96 Deep Well Real-Time PCR Detection System (Bio-Rad, Hercules, CA, USA) in a 10 $\mathrm{\mu l}$ reaction volume containing a $5 \mu$ of SYBR Green master mix (BioRad Laboratories, Philadelphia, PA, USA), $3 \mu$ of RNase-free water, $1 \mu \mathrm{l}$ of cDNA and $1 \mu \mathrm{l}$ of specific forward and reverse primers at the concentration $20 \mathrm{pmol} / \mu \mathrm{l}$. The initial denaturation step for qRT-PCR was set at $95^{\circ} \mathrm{C}$ for 4 min followed by 40 cycles of denaturation at $95^{\circ} \mathrm{C}$ for 20 seconds; annealing was at $54^{\circ} \mathrm{C}$ for 90 seconds. All samples were run in triplicate. Sequences of all primers used (Evrogen, Moscow, Russia) are listed in Supplementary Table 3. 


\section{Supplementary Table 3. Sequences of primers used for qRT-PCR}

\begin{tabular}{|c|c|c|}
\hline Gene & Forward primer 5'-3' & Reverse primer 5'-3' \\
\hline GAPDH & TGCACCACCAACTGCTTAG & GGATGCAGGGATGATGTTC \\
\hline GluA1 & GGACAACTCAAGCGTCCAGA & GTCGGTAGGAATAGCCCACG \\
\hline GluA2 & GCGTGGAAATAGAAAGGGCC & ACTCCAGTACCCAATCTTCCG \\
\hline GluA3 & ACCATCAGCATAGGTGGACTT & ACGTGGTAGTTCAAATGGAAGG \\
\hline TNF & AGCCGATGGGTTGTACCTTG & GTGGGGTGAGGAGCACGTAGTC \\
\hline IL-18 & CCTCCAGGATGAGGACATGAGCAC & TCATCATCCCATGAGTCACAGAGG \\
\hline IL-6 & TAGTCCTTCCTACCCCAATTTCC & TTGGTCCTTAGCCACTCCTTC \\
\hline IL-15 & ACATCCATCTCGTGCTACTTGT & GCCTCTGTTTTAGGGAGACCT \\
\hline GSK3B & GCACTCTTCAACTTTACCACTCA & CGAGCATGTGGAGGGATAAG \\
\hline Akt & ATGAACGACGTAGCCATTGTG & TTGTAGCCAATAAAGGTGCCAT \\
\hline
\end{tabular}

\section{Immunohistochemical analysis}

Immunohistochemical analysis was performed as described elsewhere (Vignisse et al., 2017; Pavlov et al., 2019). In order to produce paraffin blocks containing hippocampi, PFA-fixed hemispheres were incubated 2 times for $1 \mathrm{~h}$ with $70 \%$-ethanol solution, for $1.5 \mathrm{~h}$ with $80 \%$-ethanol solution, for $1 \mathrm{~h}$ with $95 \%$ ethanol solution, 3 times for $1.5 \mathrm{~h}$, in 100\% -ethanol solution, 3 times for 1.5 hour in xylene, for $2 \mathrm{~h}$, in paraffin first wax paraplast Xtra (at $58^{\circ} \mathrm{C}$ ), for $2 \mathrm{~h}$ paraffin second wax paraplast Xtra (at $58^{\circ} \mathrm{C}$ ) in cassettes (Sigma-Aldrich, St. Louis, MO, USA). Initially, a small amount of molten paraffin was dispensed in mold, 
thereafter, tissue was transferred into the mold with warm forceps. After that, the mold was placed on a cold plate for paraffin to solidify. Hot paraffin was added to the mold to cover the front surface of the plastic cassette. Then paraffin blocks were stored at the room temperature until use.

Samples were sectioned with a cryostat Leica CM 1850 cryostat (Leica Microsystems, Wetzlar, Germany) taken from lateral 3.6 to lateral $0.4 \mathrm{~mm}$ along the medial-lateral axis ahead of bregma (Paxinos and Franklin, 2001). Paraffin sections were placed on the surface of deionized water in the bath (Leica HI1210 Water bath, Wetzlar, Germany) at $40^{\circ} \mathrm{C}$. Thereafter, they were mounted onto glass slides, air dried for 30 minutes and covered with coverslips. To remove paraffin, sections were incubated 3 times for 5 min with xylene, 2 times for 10 min with 100\%-ethanol solution, 2 times for 10 min with 95\%-ethanol solution, 2 times for 10 min with $70 \%$-ethanol solution, 2 min for 10 min with $50 \%$ ethanol solution (Sigma-Aldrich, St. Louis, MO, USA) and 2 times for $5 \mathrm{~min}$ in ice-cold deionized water.

Three randomly selected hippocampal sections were incubated for 30 minutes at $90^{\circ} \mathrm{C}$ in $10 \mathrm{mM}$ sodium citrate and 0.05\% Tween 20, pH 6.0 (Applichem, Darmstadt, Germany) for antigen retrieval with GluAs. Water bath was used for heatinduced epitope retrieval. Blocking of non-specific staining was performed with $1 \%$ bovine serum albumin (BSA, Fluka, Buchs, Switzerland) in PBS, pH 7.4, at room temperature for $1 \mathrm{~h}$ with all assays. Sections were drained for a few seconds and wiped with a 
tissue paper. For surface staining, tissue was surface labelled with primary extracellular anti-GluA1/2/3 antibodies (ThermoFisher Scientific, Rockford, IL, USA, PA5-77415 / Alomone labs, Jerusalem, Israel, AGC-005-AG / Alomone labs, Jerusalem, Israel, AGC-010, respectively) overnight at $+4^{\circ} \mathrm{C}$, followed by Goat Anti-Mouse Alexa 568 secondary antibodies (ThermoFisher Scientific, Rockford, IL, USA, A-11004), Goat Anti-Mouse Alexa Fluor ${ }^{\circledR} 488$ (Abcam, Cambridge, MA, USA, ab150113), Goat anti-Mouse Alexa Fluor 405 Secondary Antibody (ThermoFisher Scientific, Rockford, IL, USA, A-31553) or Goat anti-Mouse Alexa Fluor 660 (ThermoFisher Scientific, Rockford, IL, USA, A-21074). Dilution was 1:600 for all assays.

Specificity of GluA antibodies was established in our previous experiments (Gorlova et al., 2019). Control immunofluorescence was performed in cells that express one or both proteins and specific siRNA was used to knock-down each individual protein and a loss in fluorescent staining was observed. For staining of internalized AMPARs, slices were then additionally fixed for 10 min with $4 \% \mathrm{PFA}$ and $4 \%$ sucrose for 10 min and permeabilized with 0.2\% Triton X-100 (Fluka, Buchs, Switzerland) for $1 \mathrm{~min}$ and then with $100 \%$ methanol (Fluka, Buchs, Switzerland) for 1min, after that, internalized GluAs were stained as previously reported.

For Ki67- and NeuN- staining assays, antigen retrieval was performed with three randomly selected sections incubated 3 times during 10 min per each step, in a Tris-buffered saline (TBS), 
$\mathrm{pH} 7.6$, and then treated with $2 \mathrm{~N} \mathrm{HCl}$ during 30 min at $37^{\circ} \mathrm{C}$ and washed in $0.1 \mathrm{M}$ borate buffer $\mathrm{pH} 8.5$ for $10 \mathrm{~min}$ (Applichem, Darmstadt, Germany). Water bath was used for heat-induced epitope retrieval. Blocking of non-specific staining was performed with $1 \%$ bovine serum albumin (BSA, Fluka, Buchs, Switzerland) in PBS, $\mathrm{pH}$ 7.4, at room temperature for $1 \mathrm{~h}$ with all assays. Thereafter, sections were drained for a few seconds and wiped with a tissue paper. Subsequently, overnight incubation at $+4^{\circ} \mathrm{C}$ with primary antibodies was performed using rabbit anti-Ki67 (Dako, Glostrup, Denmark) and rat polyclonal anti-NeuN neuronal marker (Dako, Glostrup, Denmark), which were diluted in TBS containing $0.1 \%$ Triton, $0.1 \%$ Tween 20 and 5\% normal donkey serum in following concentrations: Ki67 (1:100), NeuN (1:300). After that, sections were washed three times with $0.1 \%$ BSA in PBS, pH 7.4 and for 2 hours at $4^{\circ} \mathrm{C}$ in the dark incubated with donkey anti-rabbit-Alexa Fluor 647 and anti-rat-Alexa Fluor 488 secondary antibodies, respectively (Life TechnologiesMolecular Probes, Grand Island, NY, USA).

Staining with DAPI was performed to visualize cell nuclei: 10 mg of DAPI (1:1000; Santa Cruz Biotechnology, Dallas, TX, USA) was dissolved in $2 \mathrm{ml}$ of dimethylformamide (DMF), and this solution was further diluted to a concentration of $30 \mathrm{nM}$ in PBS. $350 \mu$ of this solution was used per each slice. After staining every slice was cover-slipped, incubated for $30 \mathrm{~min}$ at the room temperature and rinsed with PBS. Fluorescent microscopy was carried out with maximal fluorescence excitation/emission at 
$358 / 461 \mathrm{~nm}$. For each assay, control samples with secondary antibody alone were included for internal autofluorescence reference.

To count number of GluA puncta, dendritic structure was manually outlined in ImageJ software (NIH, Bethesda, MD, USA) by an experimenter, blind to experimental conditions; area of counting was defined as described previously (Gorlova et al., 2019) . In order to exclude the influence of cell size on counting, a quantification of GluA-positive puncta was calculated as total number of puncta per measured region of dendritic structure. Three randomly selected dendritic regions from CA3 hippocampal zone were analyzed per neuron and averaged values were calculated. Images were acquired on a Leica SP8 confocal microscope with a 63x objective (Leica Microsystems, Mannheim, Germany). Data were normalized to measures of control nonstressed vehicle-treated mice. 
Calculation of internalization index of AMPA receptor subunits

Internalization index (I) was calculated by dividing the number $(\mathrm{N})$ of GluA1-3 puncta corresponding to the internalized staining by the values corresponding to the total staining (internalized + surface) as described in the mathematical formula below:

$$
I_{\text {internalization }}=\frac{\mathbf{N}_{\text {(internalized puncta) }}}{\mathbf{N}_{\text {(surface puncta) }}+\mathbf{N}_{\text {(internalized puncta) }}}
$$

Values from ultrasound stressed group were normalized to the control group.

Images with Ki67- and NeuN- antibody staining were and processed as describe above: subgranular zone of the hippocampus was analyzed (Fig.2). SGZ was defined as a two-cellbody wide area between the hilus and the base of the granular layer of the dentate gyrus. ImageJ software (NIH, Bethesda, MD, USA) was used for counting Ki67-positive neurons. 


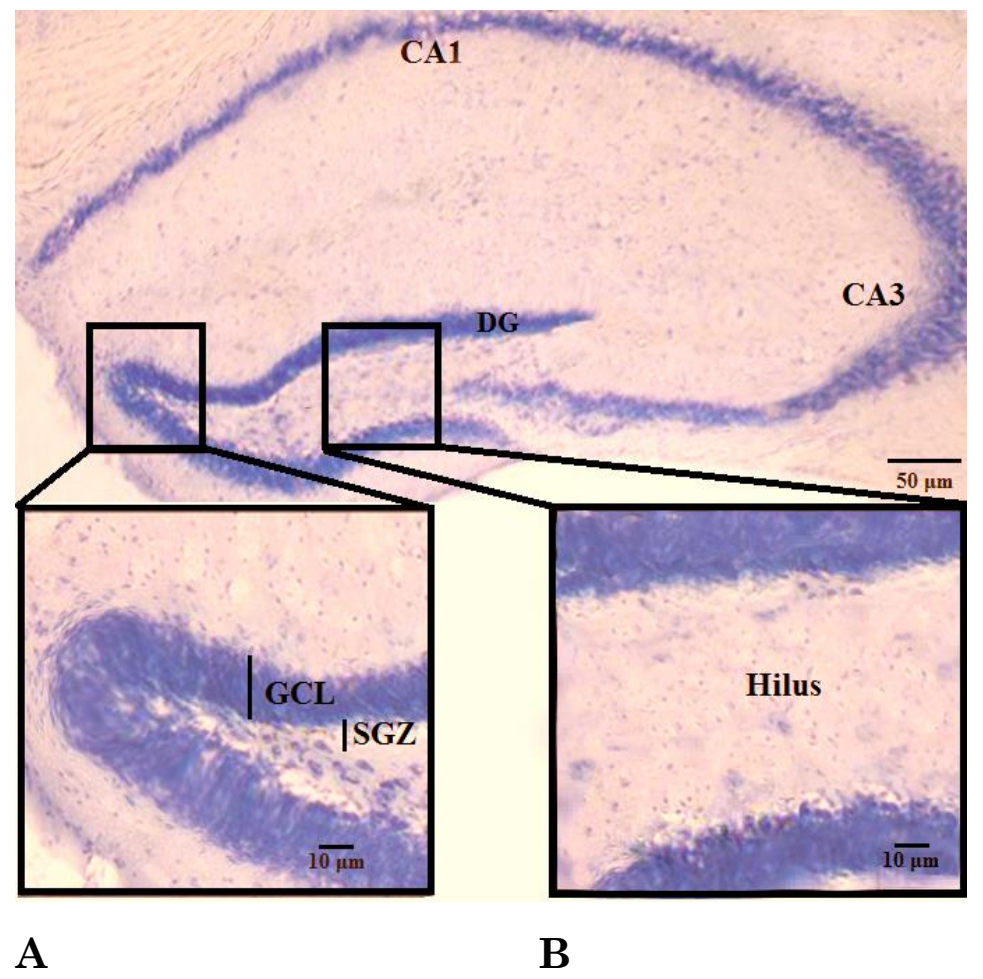

Supplementary Figure 2. Representation of the hippocampal areas defined for scoring of Ki67-positive cells. CA1 - cornu ammonis $1, \mathrm{CA} 3$ - cornu ammonis $3, \mathrm{DG}$ - dentate gyrus, GCL - granule cell layer, SGZ - subgranular zone. Scale bars are $10 \mu \mathrm{m}$ (A) and $50 \mu \mathrm{m}(\mathbf{B})$.

\section{Effects of ultrasound emission on body weight}

We found significant effect of stress $\left(\mathrm{F}_{1,24}=8.66, \mathrm{p}=0.0069\right)$, but not treatment $\left(\mathrm{F}_{2,24}=0.159, \mathrm{p}=0.854\right)$, or interaction $\left(\mathrm{F}_{2,24}=0.461, \mathrm{p}=0.636\right.$, two-way ANOVA, Fig.3) on the body weight of mice at the end of experiment. Post hoc analysis revealed significant decrease of this parameter on the day 22 in stressed non-treated mice in comparison with non-treated controls ( $p=0.0442$, Tukey's test), as well as the non-significant decrease 
of this measure in stressed mice treated with HAT-solvent in comparison with non-treated controls $(p=0.0996)$. Thus, the ultrasound exposure of mice resulted in a decrease of body weight, suggesting that applied ultrasound emission can be considered as a chronic stress model.

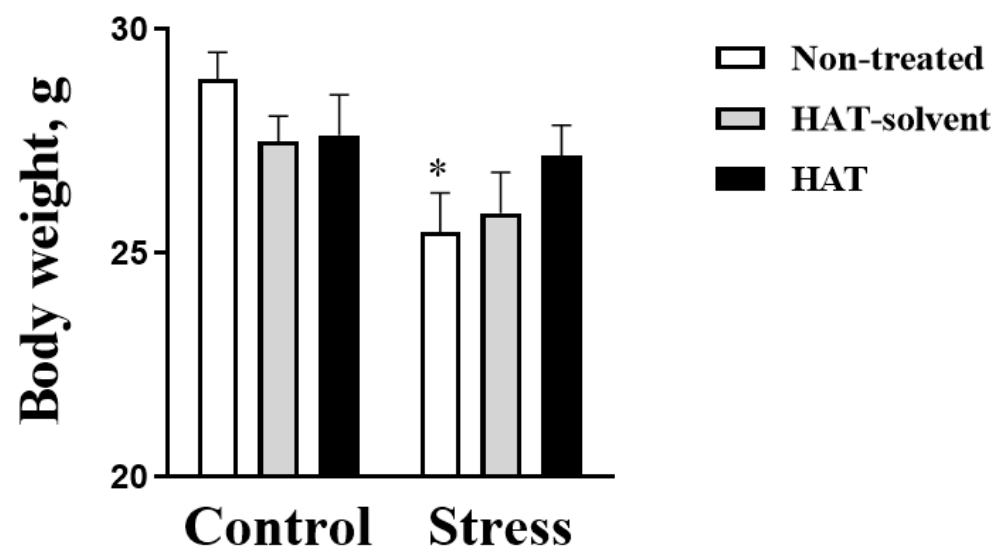

Supplementary Figure 3. Effects of ultrasound exposure and herbal treatment on body weight. There was a significant decrease in body weight in stressed non-treated mice in comparison with non-treated control group ( ${ }^{*} \mathrm{p}<0.05$, post hoc Tukey's test), 6-8 animals per group were used. HAT - herbal treatment. Bars are Mean \pm SEM.

\section{Effects of ultrasound emission on locomotor activity}

We scored total numbers of crossed sections in the O-maze and open field tests to estimate possible changes in locomotion in ultrasound-exposed mice. No significant differences were found in the number of crossed sections in the O-maze (Fig.4A), nor in the number of crossed sections in the open field (Fig.4B). 
A

O-MAZE

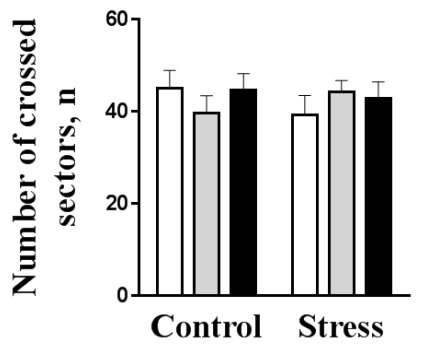

B

OPEN FIELD

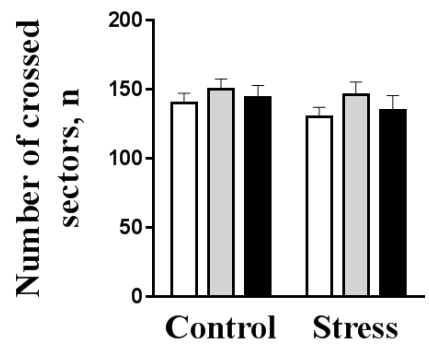

$\square$ Non-treated

$\square$ HAT-solvent

C HAT

Supplementary Figure 4. Effects of ultrasound exposure and herbal treatment on locomotor activity. There were no significant differences between groups in number of crossed sections in O-maze and open field ( $p>0.05$, post hoc Tukey's test), 6-8 animals per group were used. HAT - herbal treatment. Bars are Mean \pm SEM.

For detailed statistical analysis, see main ms text. A lack of changes in general locomotion rules out a possibility of confounds that potentially can result from prolonged exposure to stress, in behavioral analysis of mice.

Effects of ultrasound and HAT treatment on numbers NeuN- and Ki67-positive cells

Ultrasound exposure resulted in decreased number of Ki67positive cells (Fig.4G, main text and Fig.5), but did not affect number of NeuN-positive cells (Fig.5,6). For detailed statistical analysis, see main ms text. 

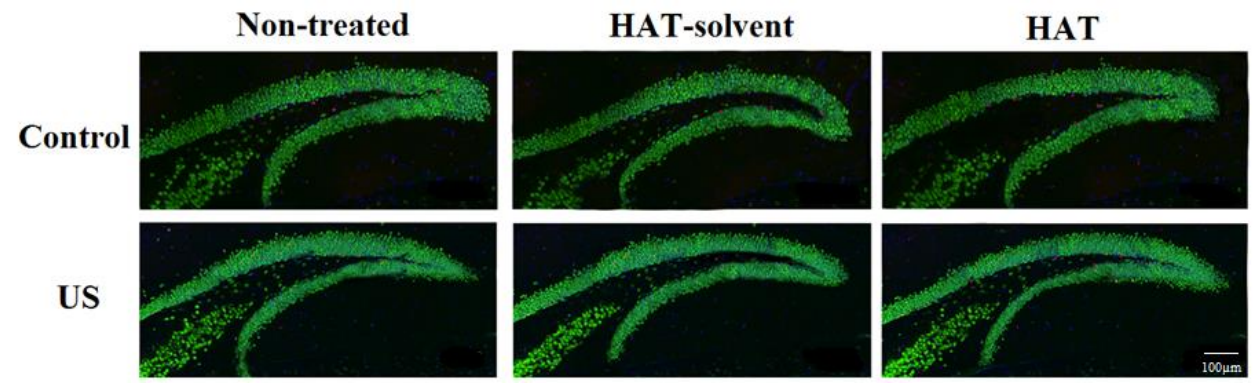

Supplementary Figure 5. Immunohistochemical analysis of Ki67-positive cells (red), NeuN-positive cells (green). Staining with DAPI was performed to visualize cell nuclei (blue). HAT - HATtreated, HAT-solvent - mice treated with HAT-solvent, US - ultrasound stress. Bar is $100 \mu \mathrm{m}$.

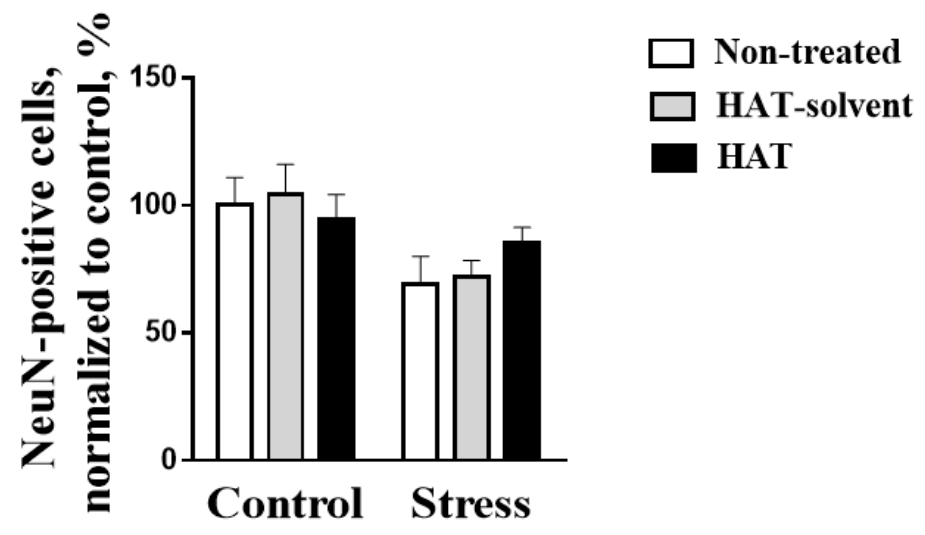

Supplementary Figure 6. Effects of ultrasound exposure and herbal treatment on the NeuN expression. There were no significant changes between groups in NeuN-positive cells in hippocampus ( $\mathrm{p}>0.05$, post hoc Tukey's test). 6-8 animals per group were used. HAT - herbal treatment. Bars are Mean \pm SEM. 


\section{Correlations between malondialdehyde content and}

measured in vivo and ex vivo endpoints in non-stressed mice

Since significant correlations were found between behavioral and biochemical parameters in all groups analyzed together, correlation analysis was additionally carried out separately in the non-stressed and stressed animals. In nonstressed mice, this analysis revealed a lack of significant correlations between malondialdehyde content and total number of entries to open arms of the O-maze ( $p=0.873, r=-0.0452$ ), time spent in open arms of $\mathrm{O}$-maze $(\mathrm{p}=0.209, \mathrm{r}=0.344)$, total number of rearings $(\mathrm{p}=0.401, \mathrm{r}=0.234)$, time spent in the center of the open field ( $\mathrm{p}=0.649, \mathrm{r}=0.111)$, latency to attack $(\mathrm{p}=0.109, \mathrm{r}=-0.412)$, total number of attacks $(\mathrm{p}=0.496, \mathrm{r}=0.191$; Fig.7A-F), relative expression of GluA2 ( $\mathrm{p}=0.638, \mathrm{r}=0.133$ ), relative expression of TNF ( $\mathrm{p}=0.396, \mathrm{r}=0.237)$, relative expression of IL-1B $(\mathrm{p}=0.283$, $\mathrm{r}=0.297)$, relative expression of GSK-3B $(\mathrm{p}=0.179, \mathrm{r}=0.366$; Fig.7G-J), internalized GluA1 puncta $(\mathrm{p}=0.764, \quad \mathrm{r}=0.085)$, internalized GluA2 puncta $(\mathrm{p}=0.971, \mathrm{r}=-0.011)$ and number of Ki67-positive cells ( $p=0.722, r=0.101$; Fig.7K-M). 
A

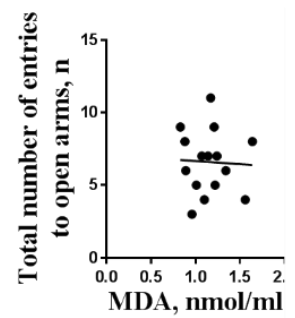

$\mathbf{E}$

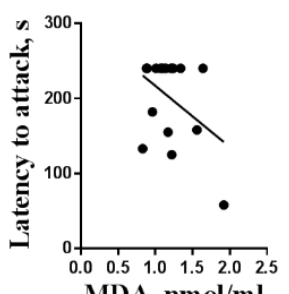

MDA, $\mathrm{nmol} / \mathrm{ml}$

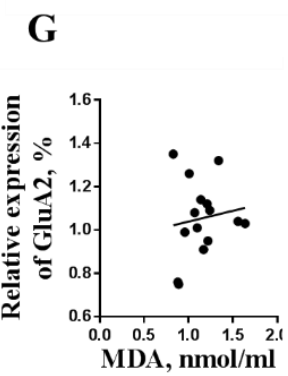

$\mathbf{K}$

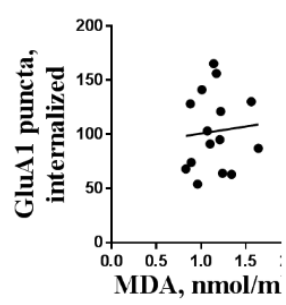

B

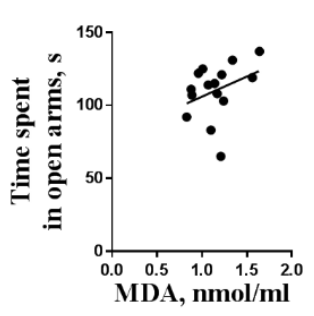

$\mathbf{F}$
C

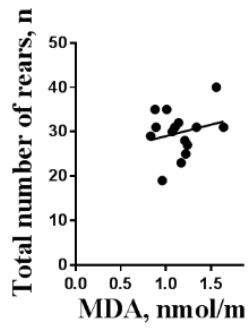

D

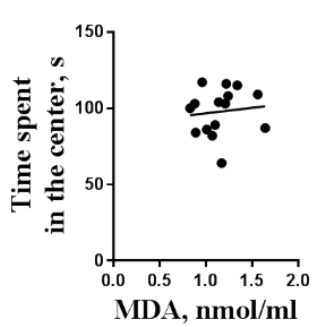

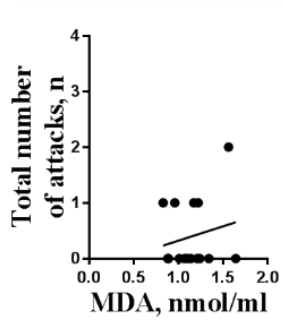

H

I

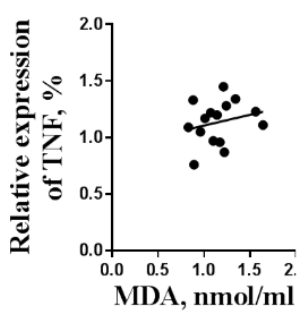

$\mathbf{L}$

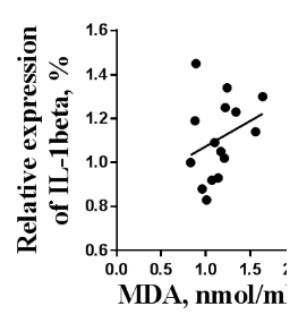

M

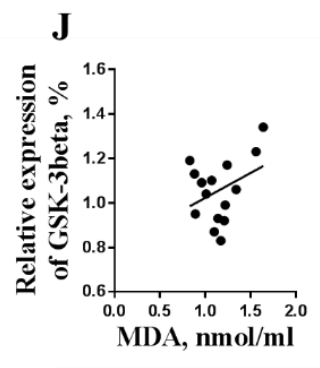

Supplementary Figure 7. Correlation between malondialdehyde content and neurobiological effects of ultrasound stress in non-stressed mice. No significant correlations were found between malondialdehyde content and (A) total number of entries to open arms of the O-maze, (B) time spent in open arms of the O-maze, (C) total number of rears, (D) time spent in the center of the 
open field, (E) latency to attack, (F) total number of attacks, (G) GluA2 mRNA expression, (H) TNF mRNA expressiom, (I) IL-1B mRNA expression, (J) relative expression of GSK-3B, (K) GluA1 internalized puncta, (L) internalized GluA2 puncta and (M) number of Ki67-positive cells ( $p>0.05$, Spearman correlation). HAT - herbal treatment.

\section{Correlations between malondialdehyde content and} measured in vivo and ex vivo endpoints in stressed mice

In stressed groups analyzed separately, significant correlations were found between MDA content and time spent in open arms of $\mathrm{O}$-maze $(\mathrm{p}=0.083, \mathrm{r}=-0.635)$, total number of rearings $(p=0.014, r=-0.619)$, time spent in the center of the open field $(p=0.002, r=-0.721)$ and latency to attack $(p<0.001, r=-0.745$; Fig.8B-E). There were significant correlations between MDA content and relative expression of GluA2 ( $\mathrm{p}=0.039, \mathrm{r}=0.518)$, relative expression of $\mathrm{TNF}(\mathrm{p}<0.001, \mathrm{r}=0.771)$ and relative expression of IL-16 ( $p=0.049, r=0.499$; Fig.8G,H,I). Finally, we found significant correlations between MDA content and internalized GluA1 puncta $(\mathrm{p}=0.024, \mathrm{r}=0.579)$ and number of Ki67-positive cells ( $\mathrm{p}=0.009$, $\mathrm{r}=-0.643$; Fig.8K,M). No correlations were found between MDA content and total number of entries to open arms of the O-maze $(\mathrm{p}=0.066, \mathrm{r}=-0.47)$, total number of attacks $(p=0.116, r=-0.409)$, relative expression of GSK3B $(\mathrm{p}=0.355, \mathrm{r}=0.248)$ and internalized GluA2 puncta $(\mathrm{p}=0.124$, $\mathrm{r}=0.415$; Fig.8G,J,L). 
$\mathbf{A}$

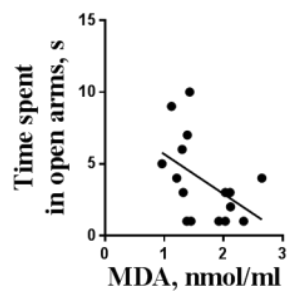

$\mathbf{E}$

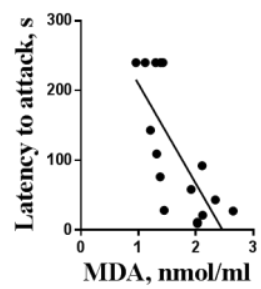

G

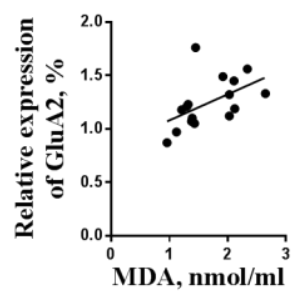

K

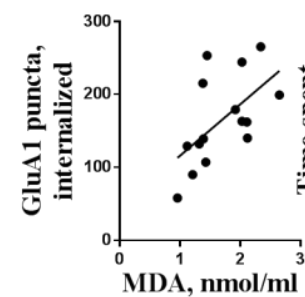

B

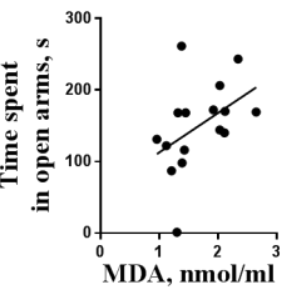

$\mathbf{F}$

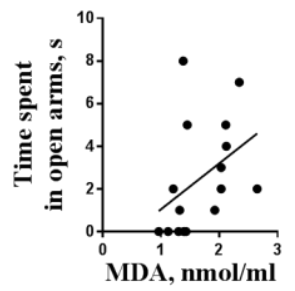

H

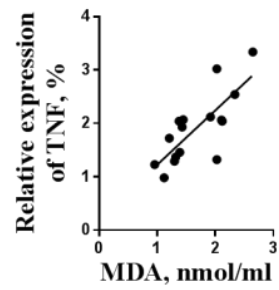

$\mathbf{L}$

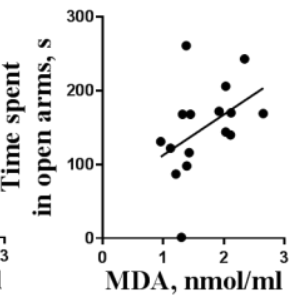

C
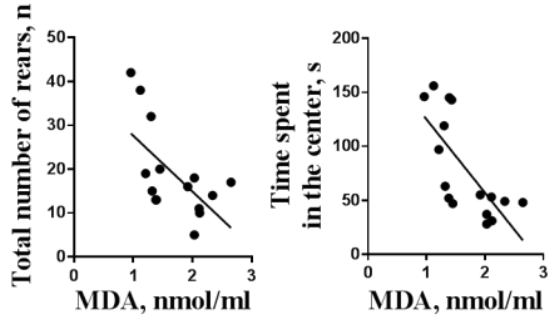

I

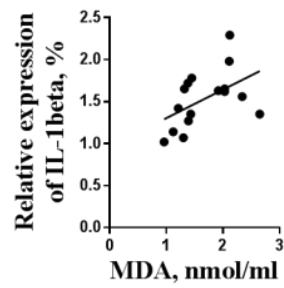

M

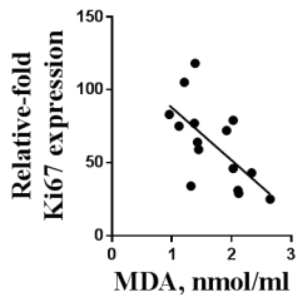

Supplementary Figure 8. Increased hippocampal content of malondialdehyde correlates with neurobiological effects of ultrasound stress in stressed mice. Significant correlations were found between malondialdehyde content and (B) time spent in open arms of the O-maze, (C) total number of rears, (D) time spent in the 
center of the open field, (E) latency to attack, (G) GluA2 mRNA expression, (H) TNF mRNA expression, (I) IL-18 mRNA expression, (K) GluA1 internalized puncta, (M) number of Ki67-positive cells $\left({ }^{*} \mathrm{p}<0.05\right.$, Spearman correlation). No significant correlations were found between malondialdehyde content and (A) total number of entries to open arms of the O-maze, (F) total number of attacks, (J) relative expression of GSK3B, (L) internalized GluA2 puncta ( $p>0.05$, Spearman correlation). HAT - herbal treatment.

\section{Correlations between number of attacks and altered}

\section{hippocampal markers}

Correlation analysis between significantly altered hippocampal markers: TNF, GSK-36, IL-1B, IL-6, and number of Ki67-positive cells (Fig.4A-D,G, main ms text) and number of attacks was performed. We found significant correlations between number of attacks in the resident-intruder test and relative gene expression of TNF ( $\mathrm{p}=0.0065, \mathrm{r}=0.478)$, IL-1B $(\mathrm{p}=0.0003, \mathrm{r}=0.608)$, GSK-3B ( $\mathrm{p}<0.0001, \mathrm{r}=0.692)$, IL6 $(\mathrm{p}=0.0059, \mathrm{r}=0.483)$ and number of Ki67-positive cells ( $p=0.047, r=-0.327$, Fig.9.A-E). 
A

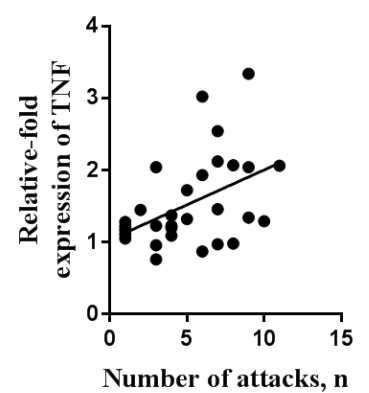

D

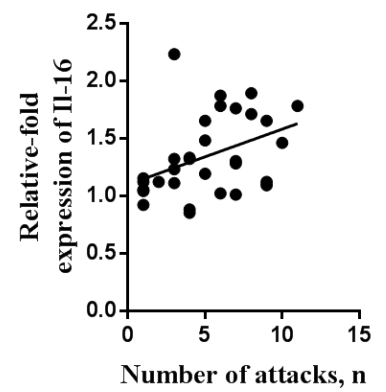

B

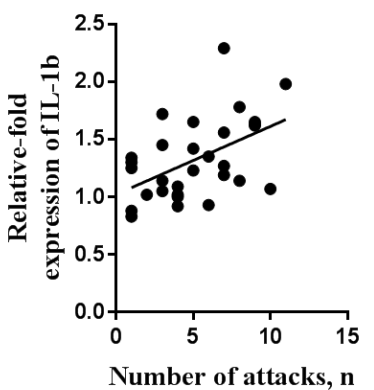

$\mathbf{E}$

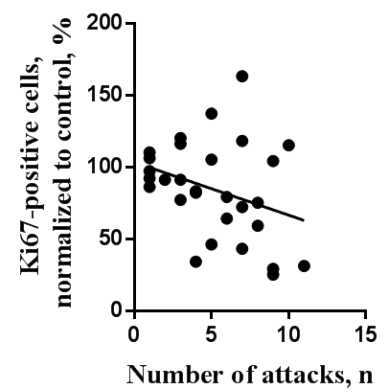

C

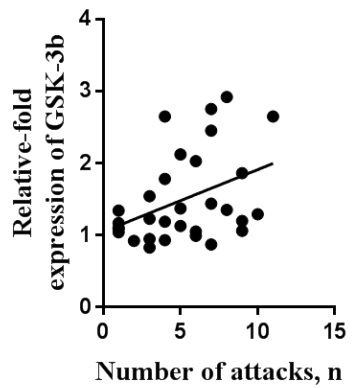

Supplementary Figure 9. Correlations between number of attacks, expression of cytokines and GSK-3ß, and number of Ki67-positive cells in stressed groups. Significant correlations were found between number of attacks and (A) relative gene expression of TNF, (B) IL-1B, (C) GSK- B, (D) IL-6, (E) number of Ki67-positive cells. 


\section{Reference list}

Ataee R., Falahati A., Ebrahimzadeh M.A., Shokrzadeh M. Anticonvulsant activities of Sambucus nigra. Eur Rev Med Pharmacol Sci. 2016; 20: 3123-3126.

Badawi M.S. Histological study of the protective role of ginger on piroxicam-induced liver toxicity in mice. J Chin Med Assoc. 2019; 82: 11-18.

Boza J.J., Turini M., Moënnoz D., Montigon F., Vuichoud J., Gueissaz N., Gremaud G., Pouteau E., Piguet-Welsch C., Finot P.A., Ballèvre O. Effect of glutamine supplementation of the diet on tissue protein synthesis rate of glucocorticoid-treated rats. Nutrition. 2001; 17: 35-40.

Chu Z.Q., Ji Q., Zhang J.L. Orally administered lemon/orange juice improved MRCP imaging of pancreatic ducts. Abdom Imaging. 2010; 35: 367-371.

Coura C.O., de Araújo I.W., Vanderlei E.S., Rodrigues J.A., Quinderé A.L., Fontes B.P., de Queiroz I.N., de Menezes D.B., Bezerra M.M., e Silva A.A., Chaves H.V., Jorge R.J., Evangelista J.S., Benevides N.M. Antinociceptive and anti-inflammatory activities of sulphated polysaccharides from the red seaweed Gracilaria cornea. Basic Clin Pharmacol Toxicol. 2012; 110: 335341.

El Mazoudy R.H., Attia A.A. Ginger causes subfertility and abortifacient in mice by targeting both estrous cycle and blastocyst implantation without teratogenesis. Phytomedicine. 2018;15: 300-308.

Frank T., Janssen M., Netzet G., Christian B., Bitsch I., Netzel M. Absorption and excretion of elderberry (Sambucus nigra L.) anthocyanins in healthy humans. Methods Find Exp Clin Pharmacol. 2007; 29: 525-533.

Gleeson M. Dosing and efficacy of glutamine supplementation in human exercise and sport training. J Nutr. 2008; 138: 2045S-2049S.

Mahmoudi M., Ebrahimzadeh M.A., Dooshan A., Arimi A., Ghasemi N., Fathiazad F. Antidepressant activities of Sambucus ebulus and Sambucus nigra. Eur Rev Med Pharmacol Sci. 2014; 18: 33503353.

Murray M., Dordevic A.L., Ryan L., Bonham M.P. The Impact of a Single Dose of a Polyphenol-Rich Seaweed Extract on Postprandial Glycaemic Control in Healthy Adults: A Randomised Cross-Over Trial. Nutrients. 2018; 10: 270. 
Nair S.A., Sr R.K., Nair A.S., Baby S. Citrus peels prevent cancer. Phytomedicine. 2018; 50: 231-237.

Neelakandan Y., Yenkatesan1 A. Antinociceptive and antiinflammatory effect of sulfated polysaccharide fractions from Sargassum wightii and Halophila ovalis in male Wistar rats. Indian J Pharmacol. 2016; 48: 562-570.

Pacheco M.T., Vezza T., Diez-Echave P., Utrilla P., Villamiel M., Moreno F.J. Anti-inflammatory bowel effect of industrial orange by-products in DSS-treated mice. Food Funct. 2018; 19: 48884896.

Stohs S.J., Preuss H.G., Shara M. A Review of the Human Clinical Studies Involving Citrus aurantium (Bitter Orange) Extract and its Primary Protoalkaloid p-Synephrine. Int J Med Sci. 2012; 9: 527-538.

Thomson M., Corbin R., Leung L. Effects of ginger for nausea and vomiting in early pregnancy: A meta-analysis. Journal of the American Board of Family Medicine. 2014; 27: 115-122.

Tiralongo E., Wee S.S., Lea R.A. Elderberry Supplementation Reduces Cold Duration and Symptoms in Air-Travellers: A Randomized, Double-Blind Placebo-Controlled Clinical Trial. Nutrients. 2016; 8: 182.

Zhang J., An J. Cytokines, Inflammation and Pain. Int Anesthesiol Clin. 2007; 45: 27-37.

Zick S.M., Djuric Z., Ruffin M.T., Litzinger A.J., Normolle D.P., Alrawi S., Feng M.R., Brenner DE. Pharmacokinetics of 6-gingerol, 8gingerol, 10-gingerol, and 6-shogaol and conjugate metabolites. Front Mol Neurosci. 2012; 5: 65. 


\section{Chapter 4}

Stress-induced aggression in

heterozygous TPH2 mutant mice is

associated with alterations in

serotonin metabolism and expression

of 5-HT6 and AMPA subunit 2A

receptors 
Chapter 4. Stress-induced aggression in heterozygous TPH2 mutant mice is associated with alterations in serotonin metabolism and expression of 5-HT6 and AMPA subunit $2 \mathrm{~A}$ receptors

Anna Gorlova ${ }^{1,2}$, Gabriela Ortega ${ }^{3}$, Jonas Waider ${ }^{3}$, Natalia Bazhenova ${ }^{1,2,4}$, Ekaterina Veniaminova ${ }^{1,2}$, Andrey Proshin ${ }^{5}$, Allan Kalueff ${ }^{6,7}$, Daniel Anthony ${ }^{8}$, Klaus-Peter Lesch ${ }^{1,2,3}$, Tatyana Strekalova $1,2,3,4$

${ }^{1}$ Laboratory of Psychiatric Neurobiology, Institute of Molecular Medicine, I.M. Sechenov First Moscow State Medical University, Moscow, Russia; ${ }^{2}$ School for Mental Health and Neuroscience, Department of Psychiatry and Neuropsychology, Maastricht University, Maastricht, The Netherlands; ${ }^{3}$ Division of Molecular Psychiatry, Center of Mental Health, University of Würzburg, Würzburg, Germany; ${ }^{4}$ Institute of General Pathology and Pathophysiology, Moscow, Russia; 5 PK Anokhin Research Institute of Normal Physiology, Moscow; ${ }^{6}$ School of Pharmacy, Southwest University, Chongqing, China; ${ }^{7}$ Institute of Translational Biomedicine, St. Petersburg State University, St. Petersburg, Russia; ${ }^{8}$ Department of Pharmacology, Oxford University, Oxford, United Kingdom.

\author{
Dr. Tatyana Strekalova \\ Department of Psychiatry and Neuropsychology \\ Maastricht University \\ Universiteitssingel 40, NL 6229 ER \\ Maastricht, Netherlands \\ Tel: 31433884 110;
}

Fax: 31433671096

email: t.strekalova@maastrichtuniversity.nl 


\section{Abstract}

Background: The contribution of gene-environment interactions that lead to excessive aggression is poorly understood. Environmental stressors and mutations of the gene encoding tryptophan hydroxylase-2 (TPH2) are known to influence aggression. For example, TPH2 null mutant mice (Tph2-/-), but not heterozygous mice (Tph2+/-), are naturally more aggressive. Here we sought to discover whether an environmental stressor would affect the phenotype of the genetically 'susceptible' heterozygous mice (Tph2+/-). Methods: Tph2+/- male mice or Tph2+/+ controls were subjected to a fiveday long rat exposure stress paradigm. Brain serotonin metabolism and the expression of selected genes encoding serotonin, AMPA receptors, and stress markers were studied. Results: Stressed Tph2+/- mice displayed increased levels of aggression and social dominance, whereas Tph2+/+ animals became less aggressive and less dominant. Concentrations of serotonin, its precursor hydroxytryptophan and its metabolite 5hydroxyindoleacetic acid were significantly altered in all groups in the prefrontal cortex, striatum, amygdala, hippocampus and dorsal raphe. Compared to non-stressed animals, the concentration of 5-hydroxytryptophan was elevated in the amygdala though decreased in the other brain structures. The overexpression of the AMPA receptor subunit, GluA2, and downregulation of 5-HT6 receptor, as well as overexpression of cfos and glycogen-synthase-kinase-3B (GSK3-B), were found in 
most structures of the stressed Tph2+/- mice. Limitations: Rescue experiments would help to verify causal relationships of reported changes. Conclusions: The interaction of a partial TPH2 gene deficit with stress results in pathological aggression and molecular changes, and suggests that the presence of genetic susceptibility can augment aggression in seemingly resistant phenotypes.

Keywords: tryptophan hydroxylase-2 (Tph2), aggression, serotonin, HPLC, AMPA receptor, 5-HT6 receptor, predator stress, mice.

\section{Introduction}

Aberrant social behaviours, particularly aggression, violence and antisocial behavior, often accompany neurodevelopmental (De Giacomo et al., 2016) and neurodegenerative (Levenson et al., 2014) conditions, as well as depression (Knox et al., 2000) and stress-related disorders (Van Voorhees et al.,2014). Aggressive behaviour during major depression is associated with an enhanced risk of suicide (Gvion \& Apter, 2011). Pathological aggression is considered to be a result of complex gene-environment interactions dependent on biological, psychological and social factors (Lesch \& Merschdorf, 2000; Lesch et al., 2012). Persistent life stress and mutations of the gene encoding tryptophan hydroxylase-2 (TPH2), the predominant enzyme required for neuronal 5-HT synthesis, are 
established as frequent causes of excessive aggression (Liu et al., 2004; Perez-Rodriguez et al., 2010; Jager et al., 2018). However, the contribution of the interaction in this pathological behavior is still poorly understood.

Aberrant 5-HT metabolism is an established factor in emotional disbalance and increased aggressiveness (Linnoila \& Virkkunen, 1992). Compromised TPH2 activity may contribute to the manifestation of these traits. Human data suggest a relation between TPH2 single nucleotide polymorphisms (SNPs) and negative emotionality (Strobel et al., 2007), as well as deficits in cognitive control and emotion regulation (Waider et al., 2011). SNPs in TPH2 were also shown to be associated with increased risk of suicidal behavior (Zhang et al., 2010) and incidence of depression (Wigner et al., 2018). In mice with complete genetic inactivation of TPH2 function (Tph2-/-), a lack of brain 5-HT results in increased aggression in the absence of an environmental stressor (Angoa-Pérez et al., 2012). Also evident is impulsivity, anxiety-related behavior and increased fear response, behavioural features often accompanying excessive aggression in animals (Coccaro et al., 2011; Neumann et al., 2009; Agis-Balboa et al., 2009). For example, aggressive Tph2-/- mice displayed signs of compulsive behaviours in the nestlet shred and the marble burying tests (Angoa-Pérez et al., 2012), and elevated scores of anxiety-like behavior in a battery of tests (Angoa-Pérez et al., 2012; Mosienko et al., 2012; Lesch et al., 2012; Gutknecht et al., 2015). Increased conditioning of fear response was found in 
Tph2-/-mice in a contextual fear conditioning paradigm (Lesch et al., 2012) and in auditory conditioning (Gutknecht et al., 2015). Parameters of aggression could be attenuated by administration of the 5-HT precursor, 5-hydroxytrypatin (5-HTP) (Angoa-Pérez et al., 2012; Mosienko et al., 2012).

The changes in aggressive and other emotional behaviors in the Tph2-l- are believed to be owing to the lack of 5-HT in the brain of the mutants, since immunofluorescent labeling confirmed the absence of specific 5-HT immunoreactivity in the raphe neurons and other brain regions of Tph2-l-mice (Gutknecht et al., 2008). Tph2-/-mice exhibit other neurochemical and molecular abnormalities, such as increased 5HT1a and $1 \mathrm{~b}$ receptor abundance and binding capacity (Gutknecht et al., 2012; Araragi et al., 2013) and a reduction in the density of GABAergic interneurons in the basolateral amygdala (Waider et al., 2019).

The 5-HT system is known to be critical in the regulation of aggression in both animals and humans. Impulsivity and high levels of aggression were found to correlate with low cerebrospinal fluid concentrations of the 5-HT metabolite 5Hydroxyindoleacetic acid (5-HIAA), in humans and nonhuman primates, and reduced 5-HT levels or metabolism in the brain of rhesus monkeys (Lesch \& Merschdorf, 2000). Genetic evidence for a role of 5-HT in aggression comes from studies on mutant mice besides Tph2-l-, which display altered 5-HT concentrations or metabolism (Tenpenny \& Commons, 2017). For example, mice 
with a knockout for voltage-dependent $\mathrm{N}$-type $\mathrm{Ca}(2+)$ channels display altered concentrations of brain 5-HT associated with excessive aggressive behaviors (Kim et al., 2009). Mice with partial or complete general transcription factor II-I repeat domain-containing protein 1 deficiency have altered 5-HT levels in the brain that correlate with changes in aggressiveness (Young et al., 2008). However, partial neuronal deficit of 5-HT may not lead to any behavioural changes in non-challenged animals. This is the case for Tph2+/- mice, which show a $20 \%$ reduction of brain 5-HT from naive controls (Mosienko et al., 2012).

Stress is known to potentiate brain 5-HT deficiency leading to a reduction in neuronal 5-HT in mice (Sachs et al., 2013), and this reduction appears to trigger aggression (Vogel \& Schwabe, 2019). Chronic and acute stress are considered the major environmental factors that interact with genetic predispositions to aggressive and violent behavior (Neumann et al., 2018). In this work, we sought to test the hypothesis of whether stress exposure combined with the partial genetic deficit of Tph (Tph2+/-) might result in a "double-hit" effect on 5-HT levels in mice and induce aggression typical for naïve Tph2-l- mice.

To pursue this goal, Tph2+/- male mice were subjected to predation stress, which has been shown to induce marked behavioural, cellular and molecular abnormalities (Strekalova et al., 2014; Vignisse et al., 2017). Social behavior, brain 5-HT metabolism, and gene expression of earlier established molecular markers of stress-induced aggression were evaluated. 
Specifically, our previous studies revealed an overexpression of AMPA receptor subunit GluA2, glycogen-synthase-kinase-3B (GSK-3B) and downregulation of 5-HT6 receptor in BALB/c mice that displayed elevated aggression scores after chronic exposure to an ultrasound model of emotional stress (Gorlova et al., 2019; Pavlov et al., 2019; Costa-Nunes et al., in press).

\section{Materials and Methods}

\subsection{Animals}

We used 10-12-week-old Tph2+/- mice, and their wild type littermates, which were bred and genotyped in the facilities at New University of Lisbon as previously described elsewhere (Gutknecht et al., 2015). 12-week-old CD1 mice were used as intruders for a resident-intruder test and 2-5-month-old Wistar rats (Charles River, Janvier, France) were used for predator stress. Tph2+/- and Tph2+/+ mice were housed individually, while CD1 mice and rats were housed in groups of five. Animals were kept under controlled laboratory conditions $\left(22 \pm 1^{\circ} \mathrm{C}, 55 \%\right.$ humidity, food and water ad libitum, lights on: 21:00 h). Studies were carried out in accordance with the European Communities Council Directive for the care and use of laboratory animals (permission 0421/000/000/2013 issued by Ethical Committee of the New University of Lisbon).

\subsection{Study outline}

Groups of mice were balanced according to their body weight, and the latency to attack was evaluated in a baseline 
resident-intruder test (Couch et al., 2016, Strekalova et al., 2018; see below). Mutants and wild type controls were subjected to a daily rat-exposure stress paradigm for 5 days (Vignisse et al.,2017; see below) and starting $24 \mathrm{~h}$ thereafter were tested in a resident-intruder test during five consecutive days, along with non-stressed groups. After 24 hours, mice were killed and their brains were isolated and dissected for subsequent HPLC and RTPCR assays (Fig.1). Two runs of the experiment were performed with an average of seven mice per group.

\subsection{Predator stress}

Mice were introduced into a spacious transparent glass cylinder $(15 \mathrm{~cm}$ high $\mathrm{x} 8 \mathrm{~cm}$ diameter) and placed into the rat cage as described elsewhere (Costa-Nunes et al., 2014; Vignisse et al., 017). 15-h exposure was performed between 18:00 and 9:00 for 5 consecutive nights. Mice had free access to food and water in their home cages between the stress sessions.

\subsection{Resident-Intruder test}

The resident-intruder test procedure was carried out during 5 consecutive days (Strekalova et al., 2004, 2018; Costa-Nunes et al., 2014; Couch et al., 2013, 2016). Mice were placed individually in an observation cage $(30 \times 60 \times 30 \mathrm{~cm})$ for $30 \mathrm{~min}$, after which a CD1 mouse was introduced. During the first 4 minutes, mice were separated by a transparent wall with holes that was removed for the following 4 minute period. The latency to follow and attack, the number and total duration of attacks and followings were 
recorded, to score aggressive and dominant behavior, in 2-min intervals (Couch et al., 2016, Gorlova et al., 2019).

\begin{tabular}{|c|c|c|c|c|c|c|c|c|}
\hline $\begin{array}{c}\text { Heterozygous mice } \\
\text { Tph } 2+/-\end{array}$ & & Days 1-5 & $+24 \mathrm{~h}$ & Days 6-10 & $+24 \mathrm{~h}$ & $\begin{array}{c}\text { Day } 11 \\
\text { Brain dissection }\end{array}$ & $\rightarrow$ & $\begin{array}{c}\text { HPLC } \\
\text { 5-HTP, 5-HT, } \\
\text { HIAA } \\
\end{array}$ \\
\hline $\begin{array}{l}\text { Wild type } \\
\text { Tph2 +/+ }\end{array}$ & $\rightarrow$ & $\begin{array}{c}\text { Rat } \\
\text { exposure } \\
\text { stress }\end{array}$ & $\rightarrow$ & $\begin{array}{c}\text { Resident- } \\
\text { Intruder } \\
\text { test }\end{array}$ & $\rightarrow$ & $\begin{array}{l}\text { Amygdala, prefrontal } \\
\text { cortex, hippocampus, } \\
\text { dorsal raphe, striatum }\end{array}$ & $\rightarrow$ & $\begin{array}{c}\text { qRT-PCR } \\
\text { 5-HT6, GluA2, } \\
\text { GSK-3 } \beta, \text { c-fos }\end{array}$ \\
\hline
\end{tabular}

Figure 1. Experiment design. Wild type and Tph2+/- mice were subjected to rat exposure stress for 5 consecutive nights, studied in 5 consecutive days in the resident-intruder test and killed. Their brained were dissected for subsequent quantitative reverse transcription polymerase chain reaction (qRT-PCR) and high performance liquid chromatography (HPLC) assays.

\subsection{Sacrifice and tissue collection}

Mice were terminally anaesthetized with an intraperitoneal injection of sodium pentobarbitone. The left ventricle was perfused with $10 \mathrm{ml}$ ice-cold saline and brains were dissected (Couch et al., 2013). The prefrontal cortex, striatum, amygdala, hippocampus and dorsal raphe were isolated and stored at $-80^{\circ} \mathrm{C}$ as described elsewhere (Gorlova et al., 2019).

2.6. High Performance Liquid Chromatography (HPLC)

The concentrations of 5-HT, its precursor 5-HTP and metabolite 5-HIAA were measured in above-listed brain regions using HPLC with electrochemical detection using the method of Waider et al., 2017 (see Supplementary material). 5-HT turnover as a ratio of 5-HT/5-HIAA was calculated. 
2.7. Quantitative reverse transcription polymerase chain reaction analysis ( $q R T-P C R)$

Total mRNA was isolated with TRI Reagent (Invitrogen, Carlsbad, CA, USA) and converted into cDNA using random primers and Superscript III transcriptase (Invitrogen, Carlsbad, CA, USA); qRT-PCR was performed using a SYBR Green master

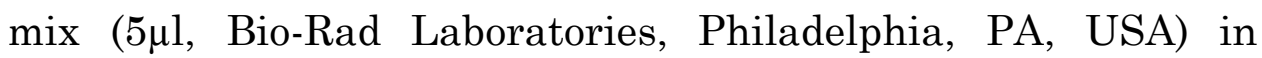
triplicate (see Supplementary material and Suppl.Table 1). Glyceraldehyde-3-phosphate dehydrogenase (GAPDH) was selected as the reference gene, and data were normalized to its expression and expressed as relative-fold changes compared to control values (Couch et al., 2016; Gorlova et al., 2019).

2.8. Statistical analysis

Data were analyzed using GraphPad Prism v.6.0 for Windows (San Diego, CA). Two-way and repeated measures ANOVA followed by post-hoc Turkey's test and Pearson correlation were applied. The level of confidence was set at $95 \%$ $(\mathrm{p}<0.05)$. 


\section{Results}

3.1. Increased aggressive behavior in stressed Tph2+/-but not Tph2+/+mice

ANOVA revealed a significant interaction between stress and genotype over the 5 days of scoring parameters of aggressive behavior: average latency to attack, average number of attacks and average duration of attacks $\left(\mathrm{F}_{3,28}=9.56, \mathrm{p}=0.005 ; \mathrm{F}_{3,28}=10.5\right.$, $\mathrm{p}=0.0034$ and $\mathrm{F}_{3,28}=5.67, \mathrm{p}=0.0259$, respectively, two-way ANOVA). All these parameters were significantly affected by stress $\left(\mathrm{F}_{3,28}=5.12, \mathrm{p}=0.0013 ; \mathrm{F}_{3,28}=4.97, \mathrm{p}=0.0081\right.$ and $\mathrm{F}_{3,28}=5.67$, $\mathrm{p}=0.0011$, respectively $)$ and genotype $\left(\mathrm{F}_{3,28}=11.12, \mathrm{p}=0.0006\right.$; $\mathrm{F}_{3,28}=7.15, \mathrm{p}=0.0023$ and $\mathrm{F}_{3,28}=5.11, \mathrm{p}=0.0321$, respectively; Fig.2A-C). Stressed Tph2+/- mice displayed decreased latency to attack and increased number and duration of attacks in comparison with non-stressed Tph2+/- mice $(\mathrm{p}=0.034, \mathrm{p}=0.0043$ and $\mathrm{p}=0.032$, respectively, Tukey's test) and stressed Tph2+/+ group ( $\mathrm{p}=0.0453, \mathrm{p}=0.029$ and $\mathrm{p}=0.009$, respectively; Fig.2A-C). The latter group had lower number of attacks than non-stressed wild type controls ( $\mathrm{p}=0.028$; Fig.2B).

There was a significant interaction between stress and genotype in the latency to attack on days 1-3 and 5, but not day 4 $\left(\mathrm{F}_{3,28}=8.12, \mathrm{p}=0.0088 ; \mathrm{F}_{3,28}=5.64, \mathrm{p}=0.0259 ; \mathrm{F}_{3,28}=5.25, \mathrm{p}=0.031 ;\right.$ $\mathrm{F}_{3,28}=5.49, \mathrm{p}=0.0295$ and $\mathrm{F}_{3,28}=0.025, \mathrm{p}=0.8757$, respectively). For days $1-5$, there was a trend to significant effects of stress on this parameter at some time point of the study $\left(\mathrm{F}_{3,28}=0.0889\right.$, $\mathrm{p}=0.7669 ; \quad \mathrm{F}_{3,28}=0.081, \quad \mathrm{p}=0.7784 ; \quad \mathrm{F}_{3,28}=0.0634, \quad \mathrm{p}=0.8033 ;$ 
$\mathrm{F}_{3,28}=3.43, \mathrm{p}=0.0763$ and $\mathrm{F}_{3,28}=0.405, \mathrm{p}=0.8425$, respectively); no significant effect of genotype was found $\left(\mathrm{F}_{3,28}=0.124, \mathrm{p}=0.728\right.$; $\mathrm{F}_{3,28}=1.69, \mathrm{p}=0.2063 ; \mathrm{F}_{3,28}=1.2, \mathrm{p}=0.2842 ; \mathrm{F}_{3,28}=3.64, \mathrm{p}=0.0685$ and $\mathrm{F}_{3,28}=0.939, \mathrm{p}=0.3441$, respectively; Fig.2D). A significant effect of stress and genotype interaction on the number of attacks was found on days 1,3 and $5\left(\mathrm{~F}_{3,28}=13.2, \mathrm{p}=0.0013 ; \mathrm{F}_{3,28}=5.92\right.$, $\mathrm{p}=0.0228 ; \mathrm{F}_{3,28}=7.97, \mathrm{p}=0.0109$, respectively) and not on days 2 and $4\left(\mathrm{~F}_{3,28}=1.15, \mathrm{p}=0.2946\right.$ and $\mathrm{F}_{3,28}=3.73, \mathrm{p}=0.066$, respectively). On days 1-5, no significant effect of stress and genotype on the number of attacks was revealed ( $>>0.05$; Fig.2E). ANOVA showed a significant stress and genotype interaction for duration of attacks on days 1 and 5 , but not on days $2-4\left(\mathrm{~F}_{3,28}=6.71, \mathrm{p}=0.016\right.$; $\mathrm{F}_{3,28}=13, \mathrm{p}=0.0015 ; \mathrm{F}_{3,28}=1.26, \mathrm{p}=0.2728 ; \mathrm{F}_{3,28}=1.04, \mathrm{p}=0.3185$ and $\mathrm{F}_{3,28}=0.801, \mathrm{p}=0.3797$, respectively). No significant effect of stress and genotype on the duration of attacks was revealed on days 1-5 ( $>>0.05$; Fig.2F).

No significant stress and genotype interaction was found in number of attacks during minutes 1-2 and 3-4 $\left(\mathrm{F}_{3,28}=0.738\right.$, $\mathrm{p}=0.4048$ and $\mathrm{F}_{3,28}=1.23, \mathrm{p}=0.2866$, respectively), as well as no significant effect of stress $\left(\mathrm{F}_{3,28}=1.51, \mathrm{p}=0.2397\right.$ and $\mathrm{F}_{3,28}=0.732$, $\mathrm{p}=0.4067$, respectively $)$, or genotype $\left(\mathrm{F}_{3,28}=2.72, \mathrm{p}=0.1213\right.$ and $\mathrm{F}_{3,28}=0.0504, \mathrm{p}=0.8257$, respectively; Fig.2G). ANOVA did not reveal any significant interaction between stress and genotype on duration of attacks during minutes $1-2$ and $3-4\left(\mathrm{~F}_{3,28}=2.45\right.$, $\mathrm{p}=0.14$ and $\mathrm{F}_{3,28}=1.7, \mathrm{p}=0.2128$, respectively, two-way ANOVA). Both stress and genotype significantly affected this measure on 
minutes 1-2 $\left(\mathrm{F}_{3,28}=4.73, \mathrm{p}=0.0473\right.$ and $\mathrm{F}_{3,28}=5.29, \mathrm{p}=0.0373$, respectively), but not $3-4\left(\mathrm{~F}_{3,28}=1.53, \mathrm{p}=0.2356\right.$ and $\mathrm{F}_{3,28}=0.549$, $\mathrm{p}=0.4708)$. Stressed Tph2+/- mice showed a significantly prolonged duration of attacks during minutes 1-2 in comparison with non-stressed Tph2+/- mice ( $\mathrm{p}=0.0286$; Fig.2H). Repeated measures ANOVA revealed a significant effect of time on the duration of attacks $\left(\mathrm{F}_{3,28}=3,928, \mathrm{p}=0,0316\right)$. There was a decrease in the duration of attacks between 3-4 minutes compared to between 1-2 minutes in stressed Tph2+/- mice $(p=0.0131$; Fig.2H), suggesting maximal expression of aggression at the beginning of the test. No such changes were found in stressed wild type mice $(\mathrm{p}=0.9901)$.

Thus, while Tph2+/- mice displayed elevated aggression scores following exposure to stress, wild type mice demonstrated rather opposing behavioural trends. These contradictions were evident after averaging measures taken across five days of testing. Remarkably, stressed Tph2+/- mice displayed increased aggressive behavior at the very first minutes of the residentintruder interaction. 


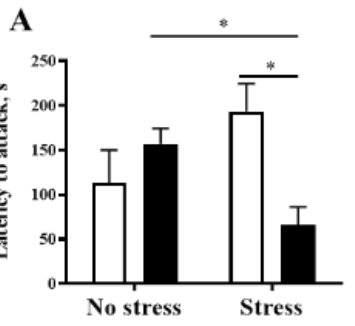

B

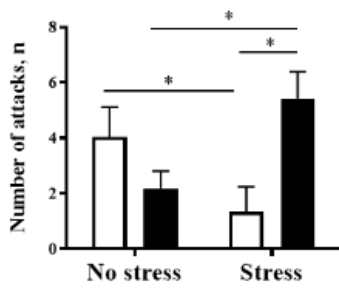

口 Wild type

$\mathrm{TPH} 2+/-$

C

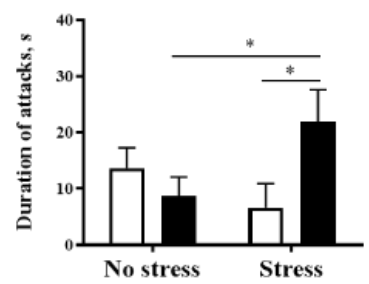

SCORES OF AGGRESSIVE BEHAVIOR, DAYS 1-5

- - Non-stressed Wild type

$\rightarrow$ Stressed Wild type

$\rightarrow$ Non-stressed TPH $2+/-$

- Stressed TPH2 +/-

\section{D}

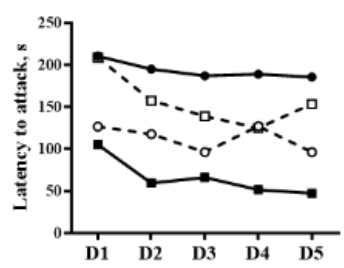

E

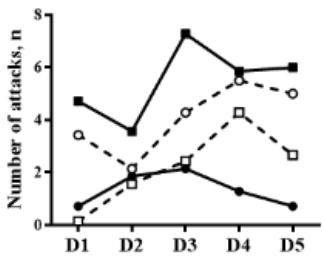

F

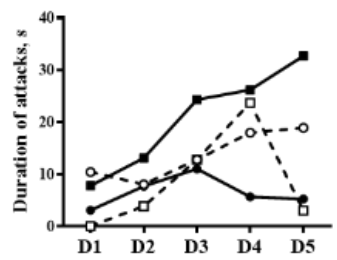

PATTERNS OF AGGRESSIVE BEHAVIOR

G

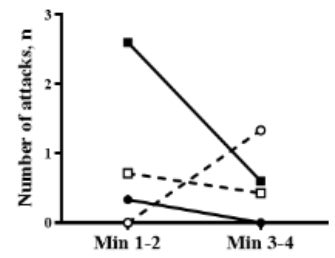

H

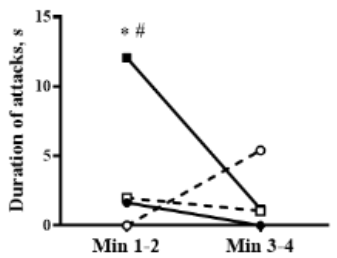

Figure 2. Opposing effects of predator stress on aggressive behavior of wild type and Tph2+/- mice. Stressed Tph2+/- mice had significantly increased aggression scores, averaged for 5 days, in comparison with non-stressed Tph2+/- mice and stressed controls, and higher number of attacks during minutes 1-2 than minutes 3-4. ${ }^{*} \mathrm{p}<0.05$, two-way ANOVA and post hoc Tukey's test. \#p<0.05 vs. Min 34. 6-9 animals per group were used. Bars represent Mean \pm SEM. 


\subsection{Increased dominant-like behavior in Tph2+/-mice}

and decreased signs of dominancy in wild type animals after stress exposure

Significant main effects of stress $\left(\mathrm{F}_{3,28}=5.23, \mathrm{p}=0.0076\right.$ and $\mathrm{F}_{3,28}=4.99 \mathrm{p}=0.046$ respectively, two-way ANOVA) and genotype $\left(\mathrm{F}_{3,28}=6.19, \mathrm{p}=0.0095\right.$ and $\mathrm{F}_{3,28}=5.08, \mathrm{p}=0.038$, respectively), as well as an interaction $\left(\mathrm{F}_{3,28}=19, \mathrm{p}=0.0004\right.$ and $\mathrm{F}_{3,28}=13.1$, $\mathrm{p}=0.0021$, respectively; Fig.3A,B) on the average latency to follow, and number of followings were found. Stressed Tph2+/-mice demonstrated lower averaged latency to follow and a higher number of followings than non-stressed Tph2+/-mice $(\mathrm{p}=0.0116$ and $\mathrm{p}=0.034$, respectively, Tukey's test) and stressed wild type animals ( $p=0.0081$ and $p=0.0405$, respectively; Fig.2A,B). In contrast, wild type animals showed prolonged averaged latency to follow in comparison with their non-stressed counterparts, as well as non-stressed Tph2+/-mice $(p=0.0369$ and $p=0.0485$, respectively; Fig.3A). They also exhibited a lower number of followings than non-stressed controls ( $p=0.0386$; Fig.3B).

There was a significant effect of stress $\left(\mathrm{F}_{3,28}=5.45, \mathrm{p}=0.0398\right.$; $\mathrm{F}_{3,28}=0.194, \quad \mathrm{p}=0.665 ; \quad \mathrm{F}_{3,28}=0.9666, \quad \mathrm{p}=0.7598 ; \quad \mathrm{F}_{3,28}=0.652$, $\mathrm{p}=0.8015$ and $\mathrm{F}_{3,28}=0.00132, \mathrm{p}=0.9716$, respectively, two-way ANOVA), genotype $\left(\mathrm{F}_{3,28}=6.12, \mathrm{p}=0.028 ; \mathrm{F}_{3,28}=0.17, \mathrm{p}=0.6853\right.$; $\mathrm{F}_{3,28}=1.08, \mathrm{p}=0.3124 ; \mathrm{F}_{3,28}=0.000288, \mathrm{p}=0.9867$ and $\mathrm{F}_{3,28}=0.454$, $\mathrm{p}=0.5123$, respectively), as well as their interaction $\left(\mathrm{F}_{3,28}=12.6\right.$, $\mathrm{p}=0.0036 ; \quad \mathrm{F}_{3,28}=0.0462, \quad \mathrm{p}=0.8325 ; \quad \mathrm{F}_{3,28}=1.87, \quad \mathrm{p}=0.1888 ;$ $\mathrm{F}_{3,28}=0.156, \mathrm{p}=0.6981$ and $\mathrm{F}_{3,28}=0.328, \mathrm{p}=0.5746$, respectively; 
Fig.2C,E) on day 5, but not days 1-4. Stressed Tph2+/-mice showed shorter latency to follow on day 5 than non-stressed Tph2+/- mice and stressed wild type animals $(\mathrm{p}=0.0203$ and $\mathrm{p}=0.0098$, respectively), while the latter group had significantly higher values of this parameter in comparison with non-stressed controls and non-stressed mutants $\quad(p=0.0102$ and $p=0.0115$, respectively; Fig.3C,E).

Significant interaction between stress and genotype in number of followings was showed on days 1-3, but not days 4-5 $\left(\mathrm{F}_{3,28}=8.12, \mathrm{p}=0.0088 ; \mathrm{F}_{3,28}=5.64, \mathrm{p}=0.0259 ; \mathrm{F}_{3,28}=5.25, \mathrm{p}=0.031\right.$; $\mathrm{F}_{3,28}=3.43, \mathrm{p}=0.0763$ and $\mathrm{F}_{3,28}=0.0405, \mathrm{p}=0.8425$, respectively, two-way ANOVA). Number of followings was significantly altered by stress and genotype on day $5\left(\mathrm{~F}_{3,28}=5.49, \mathrm{p}=0.0295\right.$ and $\mathrm{F}_{3,28}=4.86, \mathrm{p}=0.0491$, respectively), but not days 1-4 (for effects of stress: $\mathrm{F}_{3,28}=0.0899, \mathrm{p}=0.7669 ; \mathrm{F}_{3,28}=0.081, \mathrm{p}=0.7784 ; \mathrm{F}_{3,28}=0.634$, $\mathrm{p}=0.8033, \mathrm{~F}_{3,28}=0.025, \mathrm{p}=0.8757$, respectively; for effects of genotype: $\mathrm{F}_{3,28}=0.124, \mathrm{p}=0.728 ; \mathrm{F}_{3,28}=1.69, \mathrm{p}=0.2063 ; \mathrm{F}_{3,28}=1.2$, $\mathrm{p}=0.2842$ and $\mathrm{F}_{3,28}=3.64, \mathrm{p}=0.0685$, respectively; Fig.2D,F). In comparison with non-stressed Tph2+/- mice and stressed controls, stressed Tph2+/- mice displayed an elevated number of followings on day 5 ( $\mathrm{p}=0.0403$ and $\mathrm{p}=0.0193$, respectively), while stressed wild type animals demonstrated a decrease of this parameter, compared to non-stressed wild type mice and stressed Tph2+/- mice ( $\mathrm{p}=0.0385$ and $\mathrm{p}=0.0101$, respectively; Fig.3D,F). These data are evidence of the increase in dominant-like features of stressed Tph2+/- mice, further supporting the observation of 
elevated aggressiveness in these mice. At the same time, wild type mice showed a reduction of dominant-like behaviors after stress exposure.
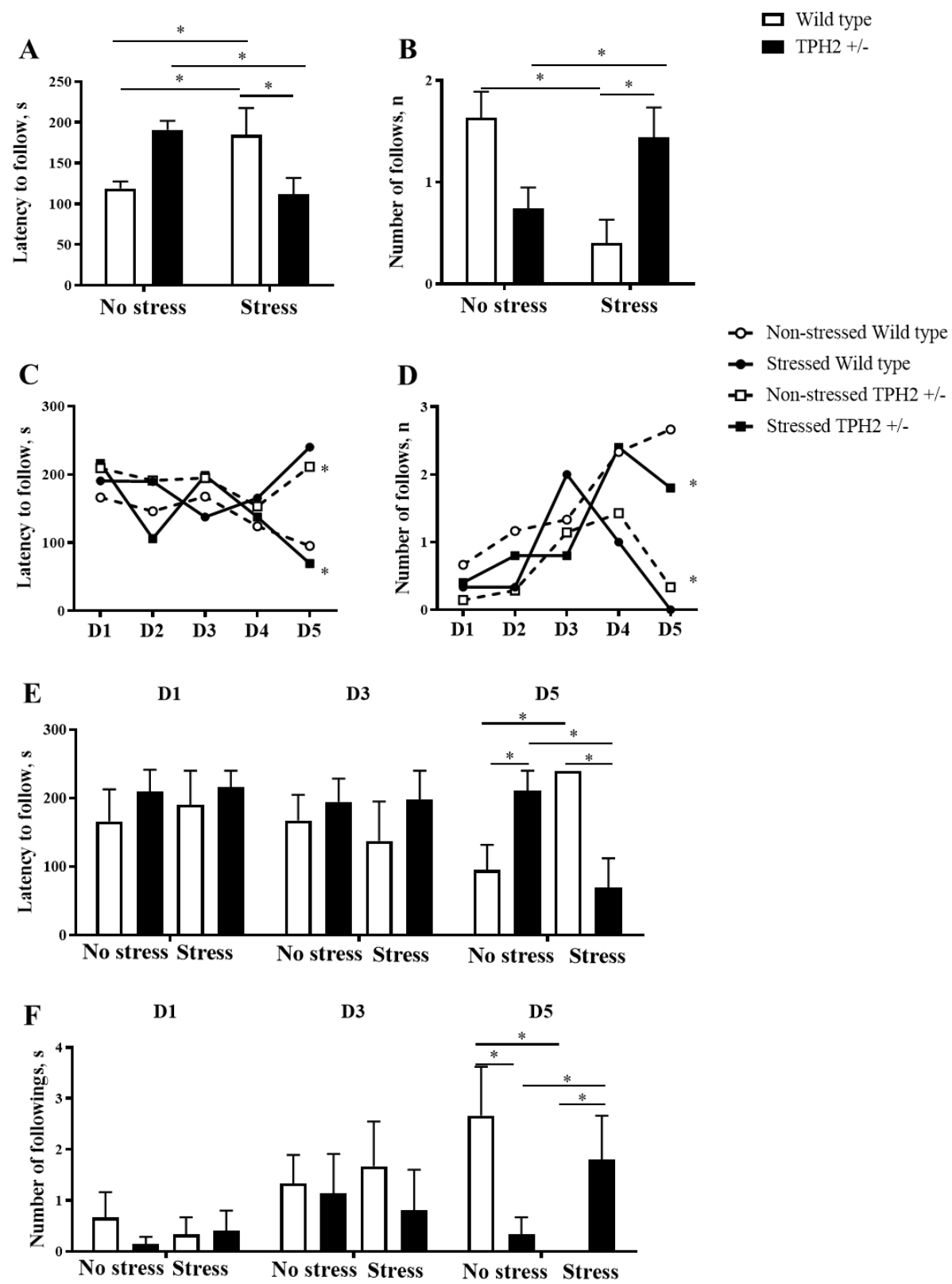
Figure 3. Differential changes in dominant-like behavior of wild type and Tph2+/-mice exposed to stress. In comparison with non-stressed Tph2+/- mice and stressed wild type group, stressed Tph2+/- mice demonstrated elevated scores of dominance, averaged for 5 days and specifically on day $5 .{ }^{*} \mathrm{p}<0.05$, two-way ANOVA and post hoc Tukey's test. 6-9 animals per group were used. Bars represent Mean \pm SEM.

\subsection{Stress-induced alterations of concentrations of 5-} HT, its precursor and 5-HT metabolite in the brain of Tph2+/- and wild type mice

We observed a significant interaction between stress and genotype for 5-HTP concentration in the prefrontal cortex, striatum and amygdala, but not in the hippocampus and dorsal raphe $\left(\mathrm{F}_{3,28}=18.9, \mathrm{p}=0.0088 ; \mathrm{F}_{3,28}=7.79, \mathrm{p}=0.0095 ; \mathrm{F}_{3,28}=81.8\right.$, $\mathrm{p}<0.0001 ; \quad \mathrm{F}_{3,28}=3.37, \mathrm{p}=0.077$ and $\mathrm{F}_{3,28}=1.12, \mathrm{p}=0.2989$, respectively, two-way ANOVA; Fig.4A,D,G,J,M). This parameter was significantly changed by stress in the prefrontal cortex, striatum, amygdala and hippocampus, but not in dorsal raphe $\left(\mathrm{F}_{3,28}=4.67, \mathrm{p}=0.0465 ; \mathrm{F}_{3,28}=133, \mathrm{p}<0.0001 ; \mathrm{F}_{3,28}=23.6, \mathrm{p}<0.0001\right.$; $\mathrm{F}_{3,28}=4.96, \mathrm{p}=0.0341$ and $\mathrm{F}_{3,28}=0.0751, \mathrm{p}=0.7861$, respectively). 5HTP concentration was also significantly affected by genotype in the prefrontal cortex, striatum, amygdala, hippocampus and dorsal raphe $\left(\mathrm{F}_{3,28}=5.26, \quad \mathrm{p}=0.0226 ; \quad \mathrm{F}_{3,28}=52.7, \quad \mathrm{p}<0.0001\right.$; $\mathrm{F}_{3,28}=10.9, \mathrm{p}=0.0028 ; \mathrm{F}_{3,28}=9.18, \mathrm{p}=0.0052$ and $\mathrm{F}_{3,28}=19, \mathrm{p}=0.0002$, respectively). Post hoc analysis revealed significant increase of this measure in the amygdala of stressed Tph2+/-mice in 
comparison with non-stressed Tph2+/- and stressed wild type mice ( $p<0.0001$ and $\mathrm{p}<0.0001$, respectively, Tukey's test; Fig.4A). Stressed Tph2+/- mice showed a decrease of this parameter in the prefrontal cortex, striatum, hippocampus and dorsal raphe $(\mathrm{p}=0.0161, \mathrm{p}<0.0001, \mathrm{p}=0.0121$ and $\mathrm{p}=0.025$ respectively; Fig.4D,G,J,M) as compared to stressed controls. 5-HTP concentration was significantly higher in the prefrontal cortex and striatum of stressed wild type mice than in non-stressed controls ( $p=0.0117$ and $p<0.0001$, respectively; Fig.4D,M).

ANOVA did not reveal significant stress and genotype interaction for 5-HT concentration in prefrontal cortex, amygdala, striatum, hippocampus and dorsal raphe $\left(\mathrm{F}_{3,28}=0.702, \mathrm{p}=0.4095\right.$; $\mathrm{F}_{3,28}=1.67, \mathrm{p}=0.2074 ; \mathrm{F}_{3,28}=2.20, \mathrm{p}=0.1496 ; \mathrm{F}_{3,28}=2.61, \mathrm{p}=0.1180$ and $\mathrm{F}_{3,28}=1.66, \mathrm{p}=0.2094$, respectively, two-way ANOVA; Fig.4B,E,H,K,N). Stress significantly affected this measure in the prefrontal cortex, striatum and dorsal raphe, but not in amygdala or hippocampus $\left(\mathrm{F}_{3,28}=9.43, \quad \mathrm{p}=0.048 ; \quad \mathrm{F}_{3,28}=13.1, \mathrm{p}=0.0012\right.$; $\mathrm{F}_{3,28}=4.80, \mathrm{p}=0.0377 ; \quad \mathrm{F}_{3,28}=3.19, \mathrm{p}=0.0853$ and $\mathrm{F}_{3,28}=0.356$, $\mathrm{p}=0.5556$, respectively), while genotype affected this parameter in the amygdala, but not in prefrontal cortex, striatum, hippocampus and dorsal raphe or $\left(\mathrm{F}_{3,28}=5.47, \mathrm{p}=0.027 ; \mathrm{F}_{3,28}=1.98\right.$, $\mathrm{p}=0.1704 ; \quad \mathrm{F}_{3,28}=2.16, \quad \mathrm{p}=0.1536 ; \quad \mathrm{F}_{3,28}=0.804, \mathrm{p}=0.3778$ and $\mathrm{F}_{3,28}=0.672, \mathrm{p}=0.4197$, respectively). In comparison with nonstressed wild type animals, 5-HT concentration was significantly decreased in the amygdala of non-stressed Tph2+/-mice $(p=0.0373$; Fig.4B) and significantly elevated in the prefrontal 
cortex and striatum of stressed wild type mice, compared to nonstressed controls ( $p=0.0422$ and $p=0.0074$, respectively; Fig.E,N).

There was a significant interaction between stress and genotype for 5-HIAA concentration in the striatum, but not in prefrontal cortex, amygdala, hippocampus and dorsal raphe $\left(\mathrm{F}_{3,28}=10.4, \quad \mathrm{p}=0.0032 ; \quad \mathrm{F}_{3,28}=0.642, \quad \mathrm{p}=0.4297 ; \quad \mathrm{F}_{3,28}=0.0631\right.$, $\mathrm{p}=80.36 ; \quad \mathrm{F}_{3,28}=2.23, \mathrm{p}=0.1467$ and $\mathrm{F}_{3,28}=2.84, \mathrm{p}=0.1041$, respectively, two-way ANOVA; Fig.4C,F,I,L,O). This parameter was significantly affected by stress in the prefrontal cortex, amygdala, striatum and hippocampus, but not in dorsal raphe $\left(\mathrm{F}_{3,28}=6.39, \mathrm{p}=0.0174 ; \mathrm{F}_{3,28}=24.5, \mathrm{p}<0.0001 ; \mathrm{F}_{3,28}=23.6, \mathrm{p}<0.0001\right.$; $\mathrm{F}_{3,28}=4.96, \mathrm{p}=0.0341$ and $\mathrm{F}_{3,28}=0.0751, \mathrm{p}=0.7861$, respectively). 5HIAA concentrations were significantly affected by genotype in the striatum, amygdala and hippocampus, but not in the prefrontal cortex and dorsal raphe $\left(\mathrm{F}_{3,28}=11.8, \mathrm{p}=0.0019\right.$; $\mathrm{F}_{3,28}=4.94, \mathrm{p}=0.0349 ; \mathrm{F}_{3,28}=6.46, \mathrm{p}=0.0171 ; \mathrm{F}_{3,28}=2.6, \mathrm{p}=0.1181$ and $\mathrm{F}_{3,28}=4.16, \mathrm{p}=0.0517$, respectively). This measure was higher in amygdala of stressed wild type mice and Tph2+/- mice than in respective non-stressed groups $(\mathrm{p}=0.0045, \mathrm{p}=0.0146$; Tukey's test Fig.4C). Stressed wild type mice displayed elevated 5-HIAA concentration in the hippocampus and striatum, compared to a non-stressed group ( $\mathrm{p}=0.0107$ and $\mathrm{p}<0.0001$, respectively) and stressed Tph2+/-mice $(\mathrm{p}=0.0429$ and $\mathrm{p}=0.0003$, respectively; Fig.4I,O). Thus, predator stress differentially altered 5-HT metabolism Tph2+/- and Tph2+/+ mice. 


\section{5-HTP}

A

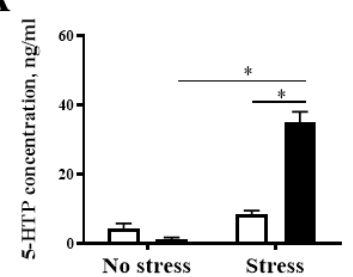

D

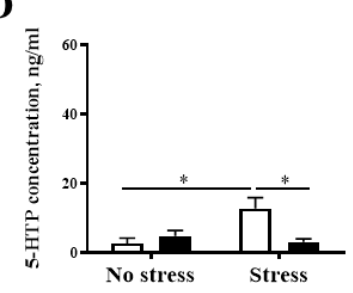

G

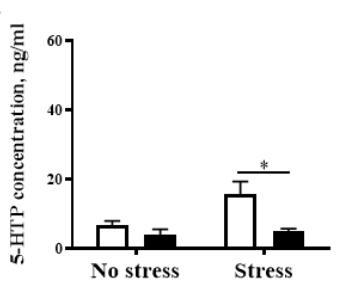

$\mathbf{J}$

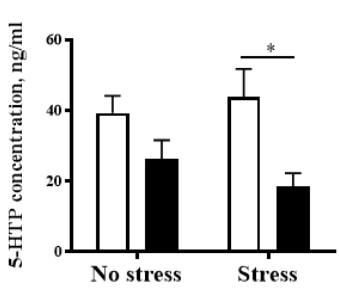

M

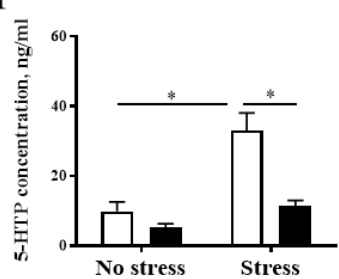

5-HT

AMYGDALA

B

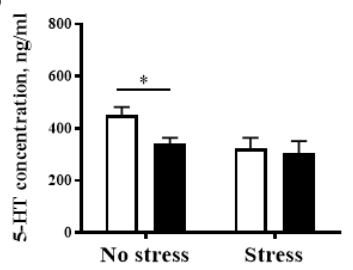

PREFRONTAL CORTEX

E

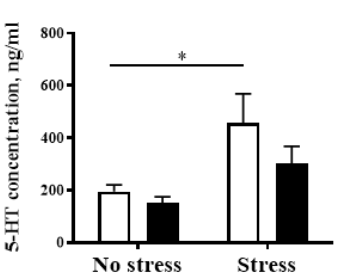

HIPPOCAMPUS

H

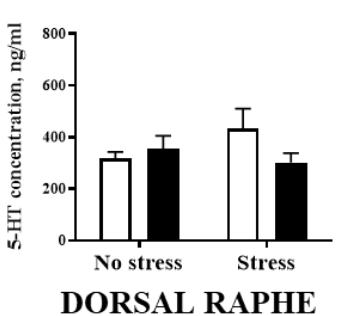

K

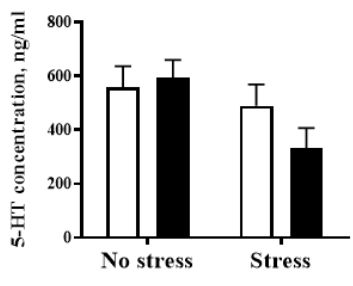

$\mathbf{N}$

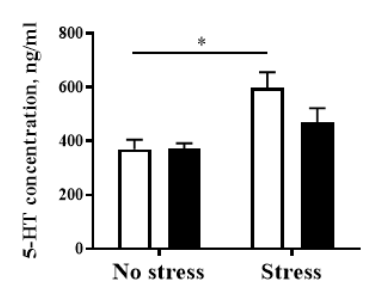

HIAA

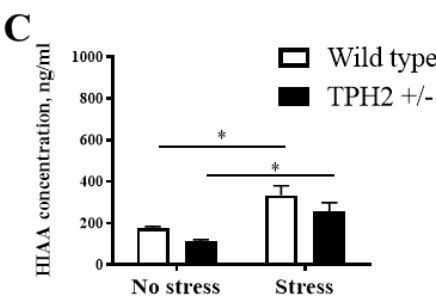

F

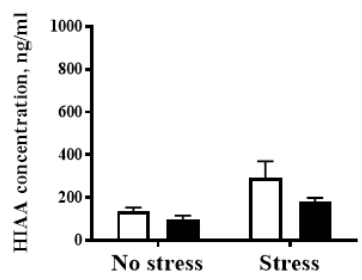

I

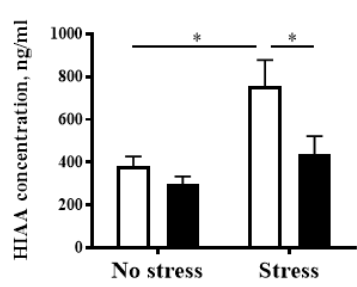

L

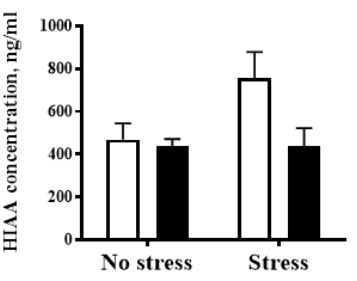

O

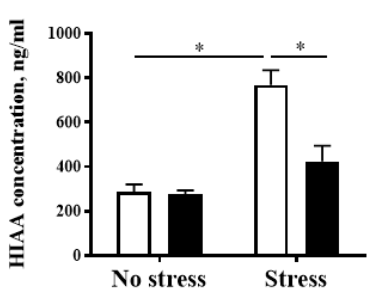

Figure 4. Stress-induced alterations of brain concentrations serotonin, its precursor and metabolite in wild type and Tph2+/- mice. Stressed Tph2+/- mice displayed 
significantly lower 5-HTP concentration than non-stressed Tph2+/mice and stressed wild type controls in all brain structures, except for the opposite effect in the amygdala. 5-HIAA and 5-HT concentration was higher in stressed wild type group than in non-stressed controls and stressed Tph2+/- in most brain structures. ${ }^{*} \mathrm{p}<0.05$, two-way ANOVA and post hoc Tukey's test. 6-9 animals per group were used. Bars represent Mean \pm SEM.

\subsection{Correlation analysis between aggression scores and 5-HT turnover in Tph2+/- and mice wild type: effects of predation}

5-HT turnover, defined as the ratio of 5-HT to 5-HIAA, was calculated for each group and brain region and relayed to the parameters of aggression/dominancy. We observed significant correlations between average number of attacks and 5-HT turnover in the prefrontal cortex of non-stressed Tph2+/- mice $(\mathrm{r}=0.86, \mathrm{p}=0.0121)$ and in the striatum of stressed wild type mice $(r=0.998, p=0.0443)$, a tendency to significant correlation between these parameters was found for stressed wild type mice in the prefrontal cortex $(\mathrm{r}=-0.99, \mathrm{p}=0.066)$ and hippocampus $(\mathrm{r}=0.27$, $p=0.0745)$. Average duration of attacks correlated significantly with 5-HT turnover in the prefrontal cortex of non-stressed Tph2+/- mice $(r=0.91, p=0.0044)$ and in the striatum of stressed wild types $(\mathrm{r}=0.998, \mathrm{p}=0.0443)$.

Average latency to follow and number of followings significantly correlated with 5-HT turnover in the striatum of stressed wild type mice $(\mathrm{r}=-0.998, \mathrm{p}=0.0443$ and $\mathrm{r}=0.998$, $\mathrm{p}=0.0443$, respectively) and in the hippocampus of non-stressed 
Tph2+/-mice $\quad(\mathrm{r}=-0.93, \quad \mathrm{p}=0.0081 \quad$ and $\mathrm{r}=0.93, \quad \mathrm{p}=0.0075$, respectively). A trend toward this correlation was observed in the prefrontal cortex of stressed wild type controls $(r=0.99, p=0.0666$ and $\mathrm{r}=-0.99, \mathrm{p}=0.066)$. Together, these data further suggest distinct neurochemical mechanisms underlying aggressive and social behaviours in stressed and naïve mice with and without genetic deficiency of TPH.

\subsection{Differences in gene expression of 5-HT receptors} and AMPA receptor GluA2 subunit between Tph2+/- mice and wild type controls: effects of stress

There was no significant interaction between stress and genotype for 5-HT6 mRNA concentration in the prefrontal cortex, striatum, amygdala, hippocampus and dorsal raphe $\left(\mathrm{F}_{3,28}=1.40\right.$, $\mathrm{p}=0.2522 ; \mathrm{F}_{3,28}=1.94, \mathrm{p}=0.1792 ; \mathrm{F}_{3,28}=1.05, \mathrm{p}=0.3189 ; \mathrm{F}_{3,28}=0.181$, $\mathrm{p}=0.675$ and $\mathrm{F}_{3,28}=0.634, \mathrm{p}=0.4358$, respectively, two-way ANOVA; Fig.5A,C,E,G,I). This measure was significantly affected by stress in the prefrontal cortex, striatum, hippocampus and dorsal raphe, but not in the amygdala $\left(\mathrm{F}_{3,28}=13.2, \mathrm{p}=0.0019\right.$; $\mathrm{F}_{3,28}=4.34, \mathrm{p}=0.048 ; \mathrm{F}_{3,28}=12.9, \mathrm{p}=0.0019 ; \mathrm{F}_{3,28}=9.74, \mathrm{p}=0.0056$; and $\mathrm{F}_{3,28}=0.0015$, $\mathrm{p}=0.9689$, respectively). Genotype significantly affected 5-HT6 mRNA levels in the striatum and hippocampus $\left(\mathrm{F}_{3,28}=4.85, \mathrm{p}=0.0439\right.$ and $\mathrm{F}_{3,28}=7.26, \mathrm{p}=0.0143$, respectively), but not in the prefrontal cortex, amygdala and dorsal raphe $\left(\mathrm{F}_{3,28}=1.85, \mathrm{p}=0.1905 ; \mathrm{F}_{3,28}=1.56, \mathrm{p}=0.2267\right.$ and $\mathrm{F}_{3,28}=0.0053$, $\mathrm{p}=0.9432$, respectively). In comparison with non-stressed 
Tph2+/- mice, stressed mutants had a decrease of this measure in the prefrontal cortex, striatum, hippocampus and dorsal raphe ( $\mathrm{p}=0.0098, \mathrm{p}=0.039, \mathrm{p}=0.0289$ and $\mathrm{p}=0.0341$, respectively, Tukey's test; Fig.5C,E,G,I). Stressed Tph2+/- mice had significantly lower 5-HT6 mRNA concentration in the striatum than stressed wild type control mice ( $\mathrm{p}=0.0402$; Fig.5I).

ANOVA showed significant stress and genotype interaction for GluA2 mRNA concentration in the prefrontal cortex and hippocampus, but not in the striatum, amygdala and dorsal raphe $\left(\mathrm{F}_{3,28}=4.93, \mathrm{p}=0.0387 ; \mathrm{F}_{3,28}=15.4, \mathrm{p}=0.0009 ; \mathrm{F}_{3,28}=1.36, \mathrm{p}=0.2632\right.$; $\mathrm{F}_{3,28}=0.009, \mathrm{p}=0.9238$ and $\mathrm{F}_{3,28}=3.28, \mathrm{p}=0.0859$, respectively, twoway ANOVA; Fig.5B,D,F,H,J). This measure was significantly affected by stress in the prefrontal cortex, hippocampus and dorsal raphe, but not in the striatum and amygdala $\left(\mathrm{F}_{3,28}=49.9\right.$, $\mathrm{p}<0.0001 ; \mathrm{F}_{3,28}=6.21, \mathrm{p}=0.0222 ; \mathrm{F}_{3,28}=9.25, \mathrm{p}=0.0067 ; \mathrm{F}_{3,28}=0.101$, $\mathrm{p}=0.7538$ and $\mathrm{F}_{3,28}=4.11, \mathrm{p}=0.0548$, respectively). Genotype affected values of GluA2 mRNA concentration in the striatum and hippocampus $\left(\mathrm{F}_{3,28}=5.2\right.$, $\mathrm{p}=0.0398 ; \mathrm{F}_{3,28}=8.3$, $\left.\mathrm{p}=0.0096\right)$, but not in the prefrontal cortex, amygdala or dorsal raphe $\left(\mathrm{F}_{3,28}=3.15\right.$, $\mathrm{p}=0.0921 ; \quad \mathrm{F}_{3,28}=0.448, \quad \mathrm{p}=0.5113$ and $\mathrm{F}_{3,28}=1.73, \quad \mathrm{p}=0.2040$, respectively). Stressed wild type controls displayed elevated GluA2 mRNA level in the prefrontal cortex in comparison with non-stressed wild types ( $\mathrm{p}=0.0226$, Tukey's test), while stressed Tph2+/- mice had this parameter decreased in comparison with non-stressed Tph2+/- mice ( $<<0.0001 ;$ Fig.5D). GluA2 expression was significantly higher in the striatum of stressed Tph2+/- mice 
than in stressed controls ( $\mathrm{p}=0.039$; Fig.5J). Stressed Tph2+/mice also demonstrated increased GluA2 expression in the hippocampus compared to non-stressed Tph2+/- mice and stressed controls ( $p=0.0005$ and $p=0.0010$, respectively; Fig.5F).

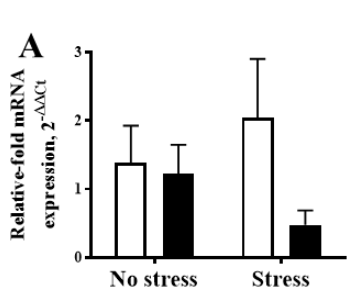

AMYGDALA

PREFRONTAL CORTEX
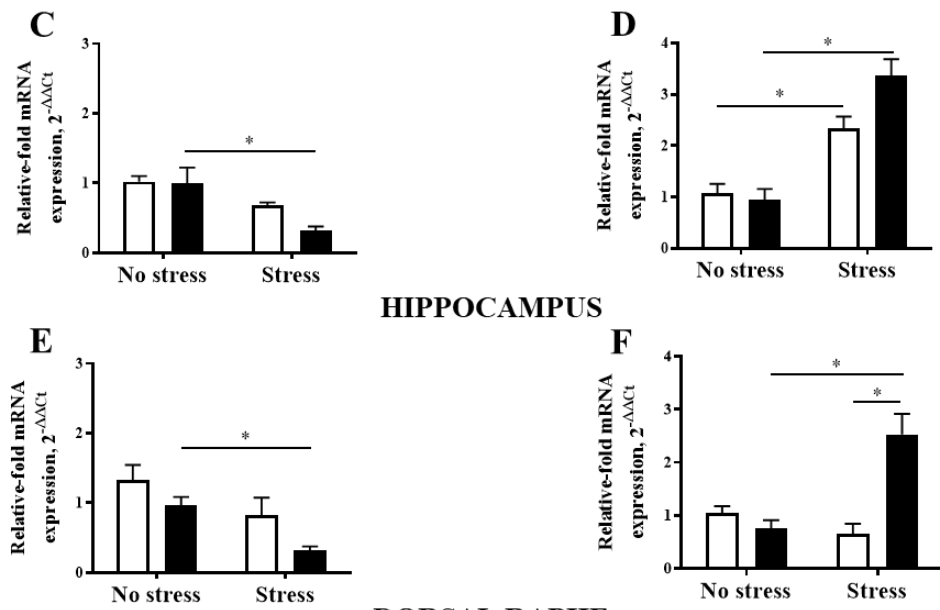

HIPPOCAMPUS
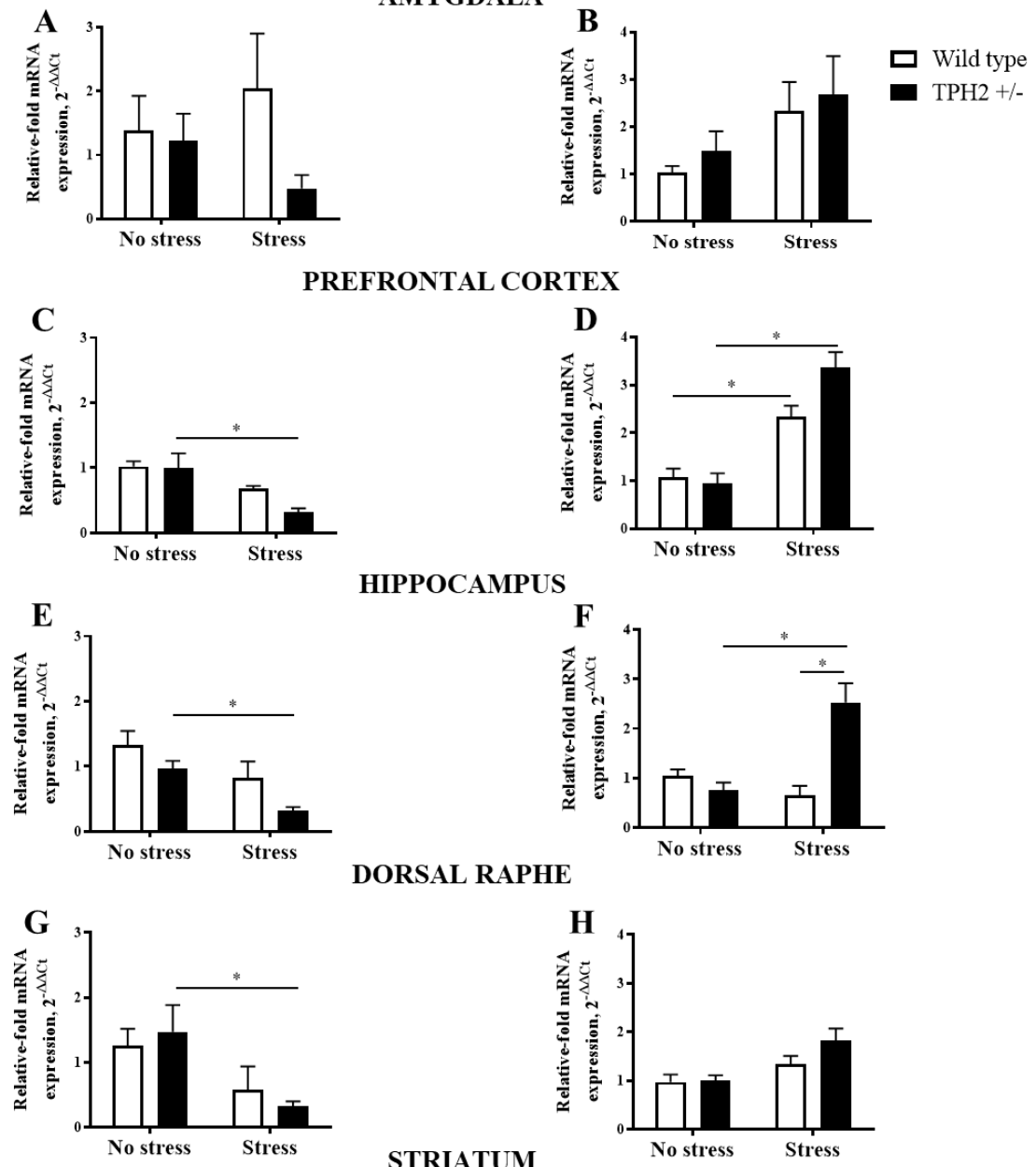

DORSAL RAPHE

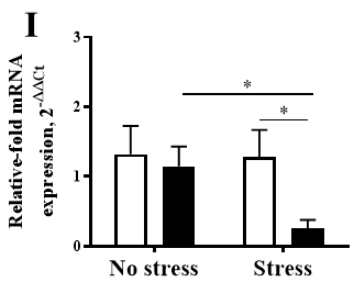

STRIATUM
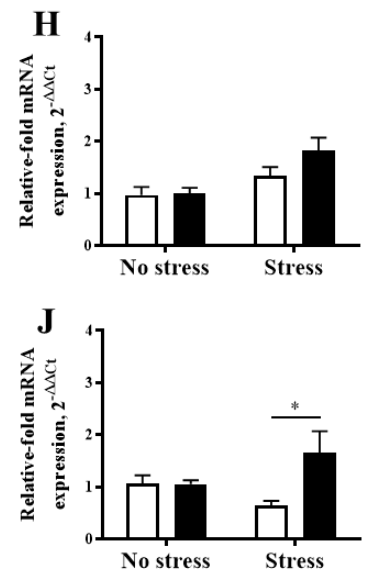
Figure 5. Stress-induced changes of the expression of 5HT6 receptor and AMPA receptor GluA2 subunit in wild type and Tph2+/- mice. Stressed Tph2+/- mice showed downregulation of 5-HT6 expression and upregulation of GluA2 expression in majority of brain structures, compared to non-stressed Tph $2+/-$ mice. * $p<0.05$, twoway ANOVA and post hoc Tukey's test. 6-9 animals per group were used. Bars represent Mean \pm SEM.

Finally, no significant effect of stress $\left(\mathrm{F}_{3,28}=0.37, \mathrm{p}=0.55\right.$ and $\mathrm{F}_{3,28}=0.781, \mathrm{p}=0.3879$, respectively, two-way ANOVA), genotype $\left(\mathrm{F}_{3,28}=0.271, \mathrm{p}=0.8711\right.$ and $\mathrm{F}_{3,28}=0.511, \mathrm{p}=0.4834$, respectively) or interaction found $\left(\mathrm{F}_{3,28}=0.0271, \mathrm{p}=0.8711\right.$ and $\mathrm{F}_{3,28}=0.121$, $\mathrm{p}=0.7321$, respectively, Suppl.Fig.2A,B) was found for mRNA concentrations of 5-HT1a and 5-HT2a receptors in the prefrontal cortex. Thus, stressed Tph2+/- mice had an overall decreased expression of the 5-HT6 receptor and an increased expression of the AMPA receptor GluA2 subunit, which in majority of cases was not found in the stressed wild type animals. At the same time, the expression of 5-HT1a and 5-HT2a receptors was unaltered.

\subsection{Altered gene expression of GSK-3ß and c-fos in}

\section{Tph2+/-and wild type mice after predator stress}

Two-way ANOVA revealed no significant interactions between stress and genotype for GSK-3B mRNA concentration in the prefrontal cortex, striatum, amygdala, hippocampus and dorsal raphe $\left(\mathrm{F}_{3,28}=0.0031, \mathrm{p}=0.9568 ; \mathrm{F}_{3,28}=0.0736, \mathrm{p}=0.7891\right.$; $\mathrm{F}_{3,28}=0.00417, \mathrm{p}=0.9492 ; \mathrm{F}_{3,28}=1.16, \mathrm{p}=0.2953$ and $\mathrm{F}_{3,28}=0.0228$, $\mathrm{p}=0.8815$, respectively, two-way ANOVA; Suppl.Fig.1A,C,E,G,I). 
Stress significantly affected this measure in the prefrontal cortex, amygdala and dorsal raphe $\left(\mathrm{F}_{3,28}=20.4, \mathrm{p}=0.0002 ; \mathrm{F}_{3,28}=5.74\right.$, $\mathrm{p}=0.0271$ and $\mathrm{F}_{3,28}=7.13, \mathrm{p}=0.0151$, respectively), but not in the striatum and hippocampus $\left(\mathrm{F}_{3,28}=0.482, \mathrm{p}=0.496\right.$ and $\mathrm{F}_{3,28}=0.293$, $\mathrm{p}=0.5943$, respectively). GSK-3B mRNA concentration was not altered by genotype in the prefrontal cortex, striatum, amygdala, hippocampus and dorsal raphe $\left(\mathrm{F}_{3,28}=0.381, \mathrm{p}=0.5446 ; \mathrm{F}_{3,28}=2.36\right.$, $\mathrm{p}=0.1411 ; \quad \mathrm{F}_{3,28}=0.885, \quad \mathrm{p}=0.3586 ; \quad \mathrm{F}_{3,28}=0.55, \quad \mathrm{p}=0.4673$ and $\mathrm{F}_{3,28}=0.0519$, $\mathrm{p}=0.8222$, respectively). Both stressed wild type and Tph2+/- mice displayed elevated GSK-3B mRNA levels in the prefrontal cortex and in the amygdala in comparison with their respective non-stressed groups $(\mathrm{p}=0.0279, \mathrm{p}=0.0116$ and $\mathrm{p}=0.0394, \mathrm{p}=0.042$, respectively, Tukey's test; Suppl.Fig.1A,C).

There was no significant stress and genotype interaction for c-fos mRNA concentration in the prefrontal cortex, striatum, amygdala, hippocampus and dorsal raphe $\left(\mathrm{F}_{3,28}=0.00326\right.$, $\mathrm{p}=0.9551 ; \quad \mathrm{F}_{3,28}=0.0775, \quad \mathrm{p}=0.3896 ; \quad \mathrm{F}_{3,28}=0.11, \quad \mathrm{p}=0.7433 ;$ $\mathrm{F}_{3,28}=1.38, \mathrm{p}=0.2552$ and $\mathrm{F}_{3,28}=1.13, \mathrm{p}=0.3003$ respectively, twoway ANOVA; Fig.1B,D,F,H,J), as well as no effect of genotype $\left(\mathrm{F}_{3,28}=0.0584, \quad \mathrm{p}=0.8116 ; \quad \mathrm{F}_{3,28}=0.567, \quad \mathrm{p}=0.4607 ; \quad \mathrm{F}_{3,28}=0.199\right.$, $\mathrm{p}=0.6604 ; \mathrm{F}_{3,28}=0.0968, \mathrm{p}=0.7592$ and $\mathrm{F}_{3,28}=0.0675, \mathrm{p}=0.7978$, respectively). c-fos mRNA concentration was significantly changed by stress in the prefrontal cortex and amygdala, but not in the striatum, hippocampus or dorsal raphe $\left(\mathrm{F}_{3,28}=19.2\right.$, $\mathrm{p}=0.0003 ; \quad \mathrm{F}_{3,28}=12.4, \quad \mathrm{p}=0.0023 ; \quad \mathrm{F}_{3,28}=0.00122, \quad \mathrm{p}=0.9725 ;$ $\mathrm{F}_{3,28}=1.34, \mathrm{p}=0.2626$ and $\mathrm{F}_{3,28}=0.0593, \mathrm{p}=0.8102$, respectively). 
Stressed wild type and stressed Tph2+/- mice showed higher cfos mRNA concentrations in the prefrontal cortex $(p=0.0374$, $\mathrm{p}=0.0145$, Tukey's test, respectively; Fig.1D) and in the amygdala $(p=0.0312, p=0.0457$, respectively; Fig.1B) than their respective non-stressed groups. Together, this suggests that the overexpression of c-fos is unlikely to underlie excessive aggression in mutants, as its expression was elevated in all stressed mice regardless of genotype.

\section{Discussion}

We found that stressed Tph2+/- mice had sharply increased parameters of aggressive and dominant-like behaviors compared to naïve Tph2+l- and wild type mice. Thus, the interaction of partial TPH2 gene deficit and stress, as hypothesized, led to pathological aggression reminiscent of the behavioural phenotype of naive TPH2 null mutants. By contrast, measures of aggressive and dominant-like behaviours in wild type controls were significantly diminished after stress. Remarkably, maximal expression of aggression in Tph2+/- mice was observed at the beginning of the interaction. Wild type mice instead displayed typical trends of increasing agonistic interactions over the course of successive social contacts. Overall, Tph2+/- mice and Tph2+/+ wild types subjected to a predator stress paradigm displayed opposing changes in aggressive and dominant behaviors with distinct dynamics of aggression expression. 
While the present study supported the hypothesis that stress induces aggressiveness in Tph2+/- mice, we did not find a further decrease in brain 5-HT in stressed Tph2+/- compared to non-stressed Tph2-/- mice. This was probably due to a ceiling effect of altered 5-HT concentrations in these animals, since this measure was significantly decreased in Tph2+/- under normal conditions. Indeed, there was a trend to a reduction in 5-HT levels in the amygdala in wild type mice that was not observed in mutants. Wild type controls exposed to stress showed significant increases of 5-HT concentrations in the prefrontal cortex and striatum that might be explained by previously reported compensatory changes (Adell et al., 1988), however this was not found in stressed mutant mice. Other studies have shown complex changes in the serotonergic system under conditions of stress, and particularly, in different subpopulations of 5-HT neurons projecting to the prefrontal cortex (Lowry et al., 2008).

Remarkably, the concentration of 5-HT precursor 5-HTP was elevated in the amygdala and decreased in other investigated brain areas of stressed Tph2+/- mice. Opposing changes were found in conventional mouse strains subjected to stress, as for instance in $\mathrm{C} 57 \mathrm{BL} / 6 \mathrm{~J}$ mice, after acute and chronic restraint stress (Browne et al., 2011). The concentration of 5-HIAA was higher in stressed controls, a change not observed in mutants. Hence, Tph2+/- and Tph2+/+ mice displayed marked differences in changes of the 5-HIAA concentration in response to stress. These data are in line with studies on mice (Browne et al., 2011) 
and macaque monkeys, subjected to early life variable foraging demand stress, where increased 5-HIAA concentrations in the cerebrospinal fluid were found (Coplan et al., 2014). Similar changes for both measures were shown in CF-1 mice exposed to the cold swim stress (Thurmond \& Brown, 1984). An increase of urinary 5-HIAA was reported in Sprague-Dawley rats after restraint stress (Kundrotas \& Gregg, 1997). In humans, stress, associated with competition, resulted in increased 5-HIAA excretion in the urine (Helin et al., 1988).

Our study revealed correlations between 5-HT turnover and aggressive behavior in both genotypes, where in mutants it was solely found in stressed mice in the amygdala. Naïve Tph2+/+ mice demonstrated such correlations in the striatum; together it can be speculated that the neuronal circuits mediating aggressive behavior in each genotype operate via distinct neuroanatomical mechanisms.

As expression of c-fos was increased in stress groups of both genotypes, and expression of GSK-36 was elevated in mutant mice regardless of stress experience, these molecular factors are unlikely to mediate excessive aggression in stressed Tph2+/-animals. Moreover, c-fos activation in Tph2+/- was previously found unrelated with functional deficits (Waider et al., 2019).

In line with previously reported findings, we showed that stress-induced aggression in Tph2+/- mice was associated with the overexpression of Glu2A and downregulation of 5-HT6 
receptor in most of the investigated brain structures (Strekalova et al., 2018; Gorlova et al., 2019; Pavlov et al., 2019). This is also in line with previously reported findings of the protein expression of GluA2 protein in mice subjected to isolation stress (Shimizu et al., 2016), and anti-anxiety effects of 5-HT6 receptor antagonist $\mathrm{ADN}-1184$ in mice and rats (Partyka et al., 2016). Notably, the 5HT6 receptor is implicated in the regulation of cognition and functionally related with activity of GABAergic neurons (de Jonk \& Mørk, 2017), whose functionality is altered in null TPH2 mutants (Waider et al., 2013; Waider et al., 2017). In human studies, allelic variant of the 5-HT6 gene was shown to be associated with aggressive behavior (Tsai et al., 1999), and 5-HT6 receptor density was significantly decreased in a cohort of patients with Alzheimer's disease that showed psychotic symptoms (Marcos et al., 2018). At the same time, the expression of 5-HT1a and 5-HT2a, whose functions were reported in TPH null mutants (Araragi et al., 2013; Jørgensen et al., 2013), were unaltered in stressed Tph2+/- mice.

Other changes previously found in Tph2 null mutant mice (Waider et al., 2013, Gutknecht et al., 2012 Angoa-Pérez et al., 2012, Gutknecht et al., 2015) might underlie stress-induced changes in aggressive behavior of mice partially deficient for TPH and are worthy of investigation. These mechanisms may involve a reduction in the density of GABAergic interneurons in the basolateral amygdala, decreased number of noradrenergic neurons in the locus coeruleus and diminished noradrenalin 
concentration in many brain regions. Additionally, a reduction in hippocampal dopamine levels, lowered plasma testosterone concentration and altered corticosterone metabolism have been found in Tph2 null mutants and should be investigated in stressed Tph2+/- mice.

\section{Conclusions}

Reported here are striking differences in aggressive / dominant behaviors between two genotypes exposed to stress. This was accompanied by differential changes of the brain metabolism of 5-HT and distinct correlations between 5-HT turnover and aggression scores across brain regions. Excessive aggression of stressed Tph2+/- mice was associated with the upregulation of AMPA receptor subunit GluA2, c-fos, GSK-3B and a downregulation of 5-HT6 receptor; the majority of these changes were not found in wild type groups. Altogether, the present study identifies molecular mechanisms of aggression, associated with a combination of TPH deficiency and stress experience. This gene environment interaction translates to a frequently encountered clinical situation. These molecular changes are in line with the factors discovered in other animal paradigms of this syndrome and highlight their use as potential targets of pharmacological management for excessive aggression. 


\section{Acknowledgments}

The authors' work reported here was supported Deutsche Forschungsgemeinschaft (DFG:CRC TRR58A1/A5), the European Union's Seventh Framework Programme (FP7/20072013) under Grant No.602805 (Aggressotype) and the Horizon 2020 Research and Innovation Programme under Grant No.728018 (Eat2beNICE) (to KPL and TS), the "5-100" Russian Academic Excellence Project (to KPL and TS) and the Russian Foundation of Basic Research (RFBR Grant No.18-34-00275 to AG). We appreciate the valuable technical help of Dr. Joao Costa-

Nunes and Dolores Bonopartos with this project.

\section{Reference list}

Adell A., Garcia-Marquez C., Armario A., Gelpi E., 1988. Chronic stress increases serotonin and noradrenaline in rat brain and sensitizes their responses to a further acute stress. J Neurochem. 50: 1678-1681.

Agis-Balboa R.C., Pibiri F., Nelson M., Pinna G., 2009. Enhanced fear responses in mice treated with anabolic androgenic steroids. Neuroreport. 20, 617-621.

Angoa-Pérez M., Kane M.J., Briggs D.I., Sykes C.E., Shah M.M., Francescutti D.M., Rosenberg D.R., Thomas D.M., Kuhn D.M., 2012. Genetic depletion of brain 5HT reveals a common molecular pathway mediating compulsivity and impulsivity. J Neurochem. 121, 974-984.

Araragi N., Mlinar B., Baccini G., Gutknecht L., Lesch K.P., Corradetti R., 2013. Conservation of 5-HT1A receptor-mediated autoinhibition of serotonin (5-HT) neurons in mice with altered 5-HT homeostasis. Front Pharmacol. 4, 97.

Browne C.A., Clarke G., Dinan T.G., Cryan J.F., 2011. Differential stress-induced alterations in tryptophan hydroxylase activity and serotonin turnover in two inbred mouse strains. Neuropharmacology. 60: 683-691. 
Coccaro E.F., Sripada C.S., Yanowitch R.N., Phan K.L., 2011. Corticolimbic function in impulsive aggressive behavior. Biol Psychiatry. 69, 1153-1159.

Coplan J.D., Fulton S.L., Reiner W., Jackowski A., Panthangi V., Perera T.D., Gorman J.M., Huang Y., Tang C.Y., Hof P.R., Kaffman A., Dwork A.J., Mathew S.J., Kaufman J., Mann J.J., 2014. Elevated cerebrospinal fluid 5-hydroxyindoleacetic acid in macaques following early life stress and inverse association with hippocampal volume: preliminary implications for serotoninrelated function in mood and anxiety disorders. Front Behav Neurosci. 8, 440.

Costa-Nunes J., Zubareva O., Araújo-Correia M., Valença A., Schroeter C.A., Pawluski J.L., Vignisse J., Steinbusch H., Hermes D., Phillipines M., Steinbusch H.M., Strekalova T., 2014. Altered emotionality, hippocampus-dependent performance and expression of NMDA receptor subunit mRNAs in chronically stressed mice. Stress. 17, 108-116.

Costa-Nunes J.P., Gorlova A., Pavlov D., Cespuglio R., Gorovaya A., Proshin A., Umriukhin A., Ponomarev E., Kalueff A., Strekalova T., Schroeter C., 2020. Ultrasound stress compromises the correlates of emotional-like states and brain AMPAR expression in mice: effects of antioxidant and anti-inflammatory herbal treatment. Stress. (in press)

Couch Y., Anthony D.C., Dolgov O., Revischin A., Festoff B., Santos A.I., Steinbusch H.W., Strekalova T., 2013. Microglial activation, increased TNF and SERT expression in the prefrontal cortex define stress-altered behaviour in mice susceptible to anhedonia. Brain Behav. Immun. 29, 136-146.

Couch Y., Trofimov A., Markova N., Nikolenko V., Steinbusch H.W., Chekhonin V., Schroeter C., Lesch K.P., 2016. Low-dose lipopolysaccharide (LPS) inhibits aggressive and augments depressive behaviours in a chronic mild stress model in mice. $\mathrm{J}$ Neuroinflammation. 13, 1-8.

De Giacomo A., Craig F., Terenzio V., Coppola A., Campa M.G., Passeri G., 2016. Aggressive Behaviors and Verbal Communication Skills in Autism Spectrum Disorders. Glob Pediatr Health. 3: 2333794X16644360.

Gorlova A., Pavlov D., Anthony D.C., Ponomarev E.D., Sambon M., Proshin A., Shafarevich I., Babaevskaya D., Lesch K.P., Bettendorff L., Strekalova T., 2019. Thiamine and benfotiamine counteract ultrasound-induced aggression, normalize AMPA 
receptor expression and plasticity markers, and reduce oxidative stress in mice. Neuropharmacology. 156: 107543.

Gutknecht L., Waider J., Kraft S., Kriegebaum C., Holtmann B., Reif A., Schmitt A., Lesch K.P., 2008. Deficiency of brain 5-HT synthesis but serotonergic neuron formation in Tph2 knockout mice. J Neural Transm (Vienna). 115, 1127-1132.

Gutknecht L., Araragi N., Merker S., Waider J., Sommerlandt F.M., Mlinar B., Baccini G., Mayer U., Proft F., Hamon M., Schmitt A.G., Corradetti R., Lanfumey L., Lesch K.P., 2012. Impacts of brain serotonin deficiency following Tph2 inactivation on development and raphe neuron serotonergic specification. PLoS One. 7: e43157.

Gutknecht L., Popp S., Waider J., Sommerlandt F.M., Göppner C., Post A., Reif A., van den Hove D., Strekalova T., Schmitt A., Colaso M.B., Sommer C., Palme R., Lesch K.P., 2015. Interaction of brain 5-HT synthesis deficiency, chronic stress and sex differentially impact emotional behavior in Tph2 knockout mice. Psychopharmacology (Berl). 232, 2429-2441.

Gvion Y., Apter A., 2011. Aggression, impulsivity, and suicide behavior: a review of the literature. Arch Suicide Res. 15, 93-112.

Helin P., Kuoppasalmi K., Laakso J., Härkönen M., 1988. Human urinary biogenic amines and some physiological responses during situation stress. Int J Psychophysiol. 6, 125-132.

Jager A., Maas D.A., Fricke K., de Vries R.B., Poelmans G., Glennon J.C., 2018. Aggressive behavior in transgenic animal models: A systematic review. Neurosci Biobehav Rev. 91, 198-217.

Jørgensen C.V., Jacobsen J.P., Caron M.G., Klein A.B., Knudsen G.M., Mikkelsen J.D., 2013. Cerebral 5-HT2A receptor binding, but not mGluR2, is increased in tryptophan hydroxylase 2 decreaseof-function mice. Neurosci Lett. 555, 118-122.

Kim C., Jeon D., Kim Y.H., Lee C.J., Kim H., Shin H.S., 2009. Deletion of $\mathrm{N}$-type $\mathrm{Ca}(2+)$ channel $\mathrm{Ca}(\mathrm{v}) 2.2$ results in hyperaggressive behaviors in mice. J Biol Chem. 284, 2738-2745.

Knox M., King C., Hanna G.L., Logan D., Ghaziuddin N., 2000. Aggressive behavior in clinically depressed adolescents. J Am Acad Child Adolesc Psychiatry. 39, 611-618.

Kundrotas L.W., Gregg R.V., 1997. Urinary excretion of 5hydroxyindoleacetic acid (5-HIAA) in the rat after immobilization stress Physiology \& Behavior. 9, 739-741.

Linnoila V.M., Virkkunen M., 1992. Aggression, suicidality, and serotonin. J Clin Psychiatry. 53, Suppl: 46-51. 
Lesch K.P., 2005. Serotonergic gene inactivation in mice: models for anxiety and aggression? Novartis Found Symp. 268, 111-140.

Lesch K.P., Merschdorf U., 2000. Impulsivity, aggression, and serotonin: a molecular psychobiological perspective. Behav Sci Law. 18, 581-604.

Lesch K.P., Araragi N., Waider J., van den Hove D., Gutknecht L., 2012. Targeting brain serotonin synthesis: insights into neurodevelopmental disorders with long-term outcomes related to negative emotionality, aggression and antisocial behaviour. Philos Trans R Soc Lond B Biol Sci. 367, 2426-2443.

Levenson R.W., Sturm V.E., Haase C.M., 2014. Emotional and behavioral symptoms in neurodegenerative disease: A model for studying the neural bases of psychopathology. Annu Rev Clin Psychol. 10, 581-606.

Liu J., 2004. Concept analysis: aggression. Issues Ment Health Nurs. 25: $693-714$.

Lowry C. A., 2002. Functional subsets of serotonergic neurones: implications for control of the hypothalamic-pituitary-adrenal axis. J. Neuroendocrinol. 14, 911-923.

Marcos B., García-Alloza M., Gil-Bea F.J., Chuang T.T., Francis P.T., Chen C.P., Tsang S.W., Lai M.K., Ramirez M.J., 2008. Involvement of an altered 5-HT-6 receptor function in behavioral symptoms of Alzheimer's disease. J Alzheimers Dis. 14, 43-50.

Mosienko V., Bert B., Beis D., Matthes S., Fink H., Bader M., Alenina N., 2012. Exaggerated aggression and decreased anxiety in mice deficient in brain serotonin. Transl Psychiatry. 2: e122.

Neumann I.D., Veenema A.H., Beiderbeck D.I., 2009. Aggression and Anxiety: Social Context and Neurobiological Links. Front Behav Neurosci. 4, 12.

Partyka A., Wasik A., Jastrzębska-Więsek M., Mierzejewski P., Bieńkowski P., Kołaczkowski M., Wesołowska A., 2016. ADN1184, a monoaminergic ligand with 5-HT6/7 receptor antagonist action, exhibits activity in animal models of anxiety. Naunyn Schmiedebergs Arch Pharmacol. 389, 593-602.

Pavlov D., Bettendorff L., Gorlova A., Olkhovik A., Kalueff A.V., Ponomarev E.D., Inozemtsev A., Chekhonin V., Lesch K.P., Anthony D.C., Strekalova T., 2019. Neuroinflammation and aberrant hippocampal plasticity in a mouse model of emotional stress evoked by exposure to ultrasound of alternating frequencies. Prog Neuropsychopharmacol Biol Psychiatry. 90, 104-116. 
Perez-Rodriguez M.M., Weinstein S., New A.S., Bevilacqua L., Yuan Q., Zhou Z., Hodgkinson C., Goodman M., Koenigsberg H.W., Goldman D., Siever L.J., 2010. Tryptophan-hydroxylase 2 haplotype association with borderline personality disorder and aggression in a sample of patients with personality disorders and healthy controls. J Psychiatr Res. 44: 1075-1081.

Sachs B.D., Rodriguiz R.M., Siesser W.M., Kenan A., Royer E.L., Jacobsen J.P.R., Wetsel W.C., Caron M.G., 2013. The effects of brain serotonin deficiency on behavioural disinhibition and anxiety-like behaviour following mild early life stress. Int $\mathrm{J}$ Neuropsychopharmacol. 16, 2081-2094.

Shimizu K., Kurosawa N., Seki K., 2016. The role of the AMPA receptor and 5-HT(3) receptor on aggressive behavior and depressive-like symptoms in chronic social isolation-reared mice. Physiol Behav. 153, 70-83.

Strekalova T., Bahzenova N., Trofimov A., Schmitt-Böhrer A.G., Markova N., Grigoriev V., Zamoyski V., Serkova T., Redkozubova O., Vinogradova D., Umriukhin A., Fisenko V., Lillesaar C., Shevtsova E., Sokolov V., Aksinenko A., Lesch k.P., Bachurin S., 2018. Pro-neurogenic, Memory-Enhancing and Anti-stress Effects of DF302, a Novel Fluorine GammaCarboline Derivative with Multi-target Mechanism of Action. Mol Neurobiol. 55, 335-349.

Strekalova T., Markova N., Shevtsova E., Zubareva O., Bakhmet A., Steinbusch H.M., Bachurin S., Lesch K.P., 2016. Individual Differences in Behavioural Despair Predict Brain GSK-3beta Expression in Mice: The Power of a Modified Swim Test. Neural Plast. 5098591.

Strekalova T., Spanagel R., Bartsch D., Henn F.A., Gass P., 2004. Stress-induced anhedonia in mice is associated with deficits in forced swimming and exploration. Neuropsychopharmacology. 29: 2007-2017.

Strobel A., Dreisbach G., Müller J., Goschke T., Brocke B., Lesch K.P., 2007. Genetic variation of serotonin function and cognitive control. J Cogn Neurosci. 19, 1923-1931.

Thurmond J.B., Brown J.W., 1984. Effect of brain monoamine precursors on stress-induced behavioral and neurochemical changes in aged mice. Brain Res. 296, 93-102.

Tenpenny R.C., Commons K.G., 2017. What Gene Mutations Affect Serotonin in Mice? ACS Chem Neurosci. 8, 987-995. 
Tsai S.J., Chiu H.J., Wang Y.C., Hong C.J., 1999. Association study of serotonin-6 receptor variant (C267T) with schizophrenia and aggressive behavior. Neurosci Lett. 271, 135-137.

van Donkelaar E.L., Blokland A., Lieben C.K., Kenis G., Ferrington L., Kelly P.A., Steinbusch H.W., Prickaerts J., 2010. Acute tryptophan depletion in C57BL/6 mice does not induce central serotonin reduction or affective behavioural changes. Neurochem Int. 56, 21-34.

Van Voorhees E.E., Dennis P.A., Elbogen E.B., Clancy C.P., Hertzberg M.A., Beckham J.C., Calhoun P.S., 2014. Personality assessment inventory internalizing and externalizing structure in veterans with posttraumatic stress disorder: associations with aggression. Aggress Behav. 40, 582-592.

Vignisse J., Sambon M., Gorlova A., Pavlov D., Caron N., Malgrange B., Shevtsova E., Svistunov A., Anthony D.C., Markova N., Bazhenova N., Coumans B., Lakaye B., Wins P., Strekalova T., Bettendorff L., 2017. Thiamine and benfotiamine prevent stressinduced suppression of hippocampal neurogenesis in mice exposed to predation without affecting brain thiamine diphosphate levels. Mol Cell Neurosci. 82, 126-136.

Vogel S., Schwabe L., 2019. Stress, aggression, and the balance of approach and avoidance. Psychoneuroendocrinology. 103, 137146.

Waider J., Proft F., Langlhofer G., Asan E., Lesch K.P., Gutknecht L., 2013. GABA concentration and GABAergic neuron populations in limbic areas are differentially altered by brain serotonin deficiency in Tph2 knockout mice. Histochem Cell Biol. 139, 267 281.

Waider J., Araragi N., Gutknecht L., Lesch K.P., 2011. Tryptophan hydroxylase-2 (TPH2) in disorders of cognitive control and emotion regulation: a perspective.

Psychoneuroendocrinology. 36, 393-405.

Waider J., Popp S., Mlinar B., Montalbano A., Bonfiglio F., Aboagye B., Thuy E., Kern R., Thiel C., Ararag N., Svirin E., Schmitt-Böhrer A.G., Corradetti R., Lowry C.A., Lesch K.P., 2019. Serotonin deficiency increases context-dependent fear learning through modulation of hippocampal activity. Front Neurosci. 13: 245.

Waider J., Popp S., Lange M.D., Kern R., Kolter J.F., Kobler J., Donner N.C., Lowe K.R., Malzbender J.H., Brazell C.J., Arnold M.R., Aboagye B., Schmitt-Böhrer A., Lowry C.A., Pape H.C., Lesch K.P., 2017. Genetically driven brain serotonin deficiency 
facilitates panic-like escape behavior in mice. Transl Psychiatry. 7(10): e1246.

Wigner P., Czarny P., Synowiec E., Bijak M., Białek K., Talarowska M., Galecki P., Szemraj J., Sliwinski T., 2018. Association between single nucleotide polymorphisms of TPH1 and TPH2 genes, and depressive disorders. J Cell Mol Med. 22, 1778-1791.

Young E.J., Lipina T., Tam E., Mandel A., Clapcote S.J., Bechard A.R., Chambers J., Mount H.T.J., Fletcher P.J., Roder J.C., Osborne L.R., 2008. Reduced fear and aggression and altered serotonin metabolism in Gtf2ird1-targeted mice. Genes Brain Behav. 7, 224-234.

Zhang Y.Q., Zhang C.S., Yuan G.Z., Yao J.J., Cheng Z.H., Liu C.J., Liu Q.H., Wan G.R., Shi G.Z., Cheng Y.R., Ling Y., Li K., 2010. Effect of Tryptophan Hydroxylase-2 rs7305115 SNP on suicide attempts risk in major depression. Behav Brain Funct. 6, 49.

\section{Supplementary material}

\section{High Performance Liquid Chromatography (HPLC)}

Brain tissue was briefly sonicated in $0.1 \mathrm{M}$ perchloric acid (PCA) with antioxidants (1 mM Na2S2O5, $0.215 \mathrm{mM} \mathrm{Na2-EDTA)}$ (about 1:20 w/v) and centrifuged at $20.627 \mathrm{~g}$ for $20 \mathrm{~min}$ at $4 \mathrm{C}$. Supernatant was injected into a HPLC system (L-7100 pump, L7200 autosampler and interface D-7000, Merck-Hitachi) and pumped through a Waters Spherisorb S5 ODS2 guard column (4.6 x $30 \mathrm{~mm}$ ) and a Waters Spherisorb S3 ODS2 cartridge analytical column (4.6 x $150 \mathrm{~mm}$, Waters, MA, USA). The electrochemical detector (Decade, Antec, Leyden) was set to an oxidation potential of b0.75 V against an $\mathrm{Ag} / \mathrm{AgCl}$ reference electrode. We used a mobile phase consisting of $0.1 \mathrm{M}$ sodium acetate, $6.0 \%$ methanol, $0.07 \mathrm{mM}$ n-octyl sodium sulfate and $0.034 \mathrm{mM}$ Na2-EDTA 
dissolved in Milli-Q water, adjusted to $\mathrm{pH} 4.1$ with glacial acetic acid.

Quantitative reverse transcription polymerase chain reaction (qRT-PCR) method and analysis

Total mRNA was isolated with TRI Reagent (Invitrogen, Carlsbad, CA, USA). 1 $\mathrm{\mu g}$ of total RNA was converted into cDNA using random primers and Superscript III transcriptase (Invitrogen, Carlsbad, CA, USA). qRT-PCR was performed in a 10 $\mu \mathrm{l}$ reaction volume containing a SYBR Green master mix (5 $\mu \mathrm{l}$, Bio-Rad Laboratories, Philadelphia, PA, USA). All qRT-PCR samples were run on a CFX96 Deep Well Real-Time PCR Detection System (Bio-Rad, Hercules, CA, USA) in triplicate (see Supplementary material and Supplementary Table 1). The housekeeping gene glyceraldehyde-3-phosphate dehydrogenase (GAPDH) was selected as the reference following previously established protocols (Pavlov et al., 2019). Data were normalized to GAPDH mRNA expression and calculated as relative-fold changes compared to control mice as described elsewhere (Couch et al., 2016; Gorlova et al., 2019).

qRT-PCR was performed using the CFX96 Deep Well RealTime PCR Detection in a $10 \mu \mathrm{l}$ reaction volume containing a $5 \mu$ of SYBR Green master mix, $3 \mu$ of RNase-free water, $1 \mu \mathrm{l}$ of cDNA and $1 \mu \mathrm{l}$ of specific forward and reverse primers at the concentration $20 \mathrm{pmol} / \mu \mathrm{l}$. The initial denaturation step for qRT- 
PCR was set at $95^{\circ} \mathrm{C}$ for 4 min followed by 40 cycles of denaturation at $95^{\circ} \mathrm{C}$ for 20 seconds. Annealing was at $54^{\circ} \mathrm{C}$ for 90 seconds. Sequences of all primers used (Evrogen, Moscow, Russia) are listed in Supplementary Table 1.

Supplementary Table 1. Sequences of primers used for qRT-PCR

\begin{tabular}{|c|c|c|}
\hline Gene & Forward primer 5'-3' & Reverse primer 5'-3' \\
\hline GAPDH & TGCACCACCAACTGCTTAG & GGATGCAGGGATGATGTTC \\
\hline 5-HT6 & GCATAGCTCAGGCCGTATGT & CACCACTGTGAGAGGTCCAC \\
\hline 5-HT1a & GACAGGCGGCAACGATACT & CCAAGGAGCCGATGAGATAGTT \\
\hline 5-HT2a & TAATGCAATTAGGTGACGACTCG & GCAGGAGAGGTTGGTTCTGTTT \\
\hline GluA2 & GCGTGGAAATAGAAAGGGCC & ACTCCAGTACCCAATCTTCCG \\
\hline GSK38 & GCACTCTTCAACTTTACCACTCA & CGAGCATGTGGAGGGATAAG \\
\hline c-fos & CGGGTTTCAACGCCGACTA & TTGGCACTAGAGACGGACAGA \\
\hline
\end{tabular}

\footnotetext{
Effects of predator stress on expression of GSK-3 $\beta$ and c-fos in wild type and Tph2+/- mice

Predator stress resulted in increased expression of GSK-3B and c-fos in the prefrontal cortex and hippocampus of stressed animals (Suppl.Fig.1A-D) but did not affect these parameters in other brain structures (Suppl.Fig.1E-J). For detailed statistical analysis, see main text.
} 

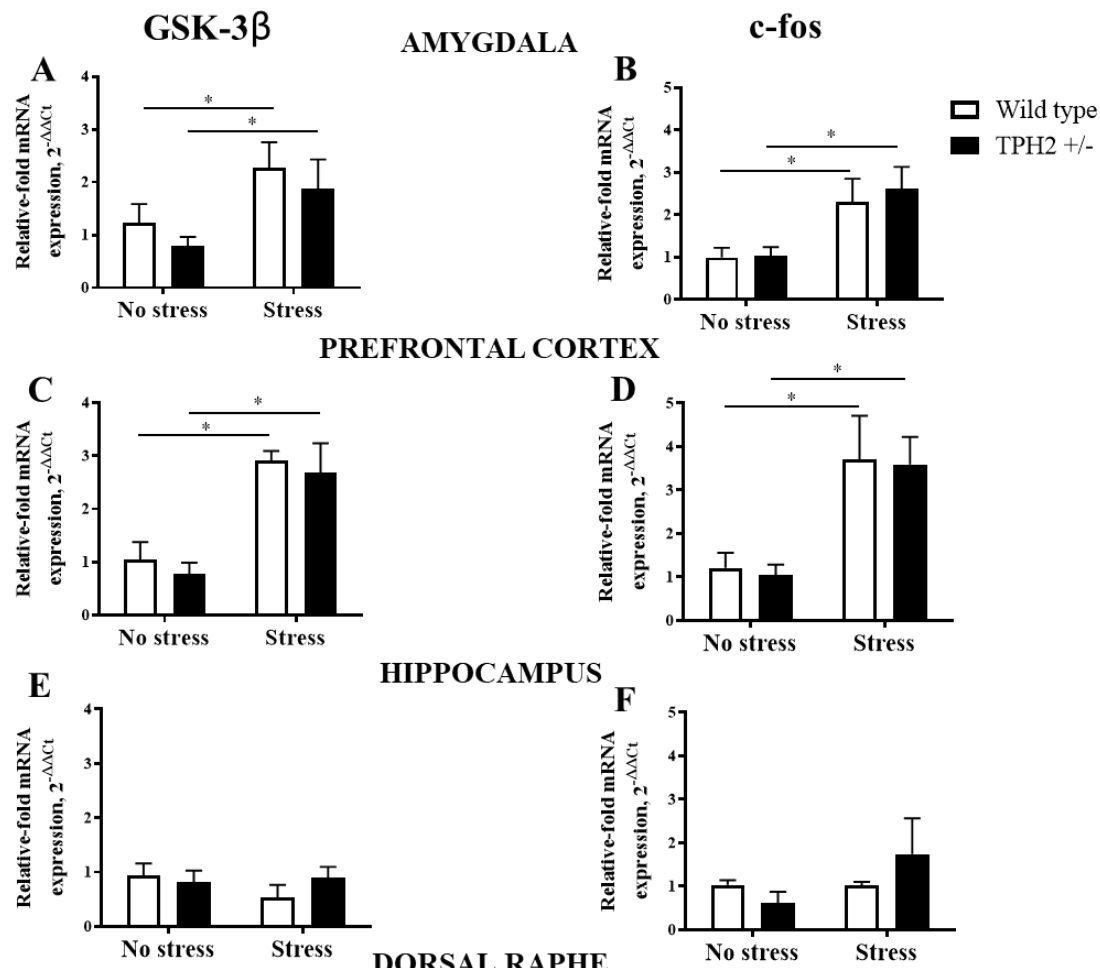

HIPPOCAMPUS
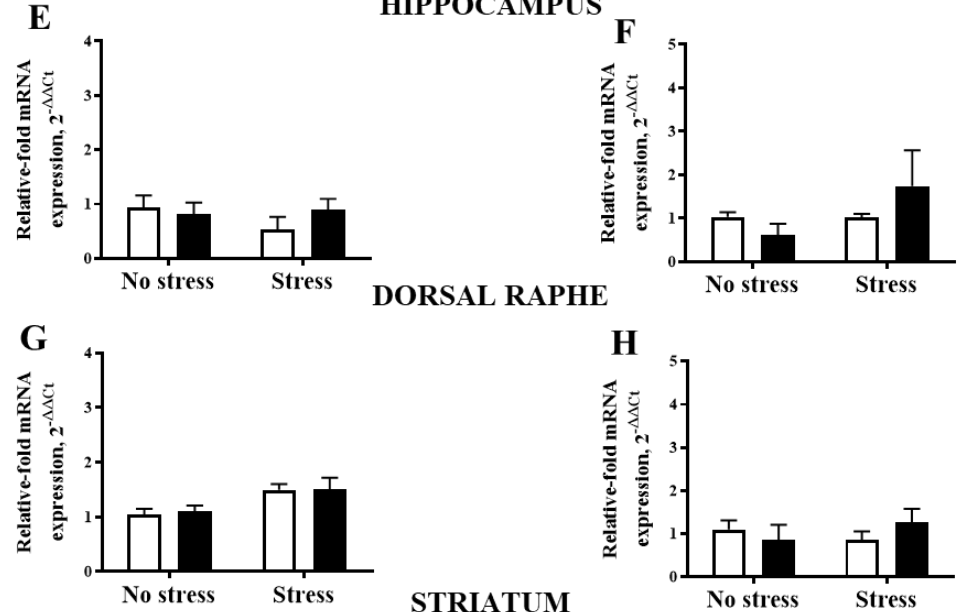

ORSAL RAPHE
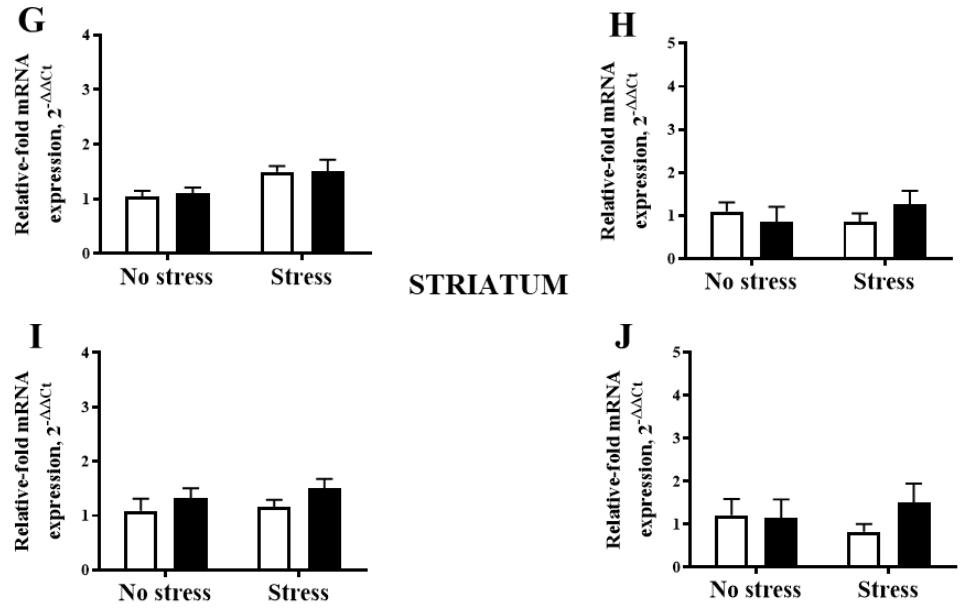

Supplementary Figure 1. Effects of predator stress on expression of GSK-3 $\beta$ and c-fos in wild type and Tph2+/- mice. (A) Stressed wild type mice had increased GSK-36 mRNA level in comparison with non-stressed wild types, and stressed Tph2+/- mice had this measure increased, compared to non-stressed Tph2+/- mice in 
amygdala and (C) in the prefrontal cortex. (B) Stressed wild type mice showed higher c-fos expression than non-stressed wild types, and stressed Tph2+/- mice demonstrated higher c-fos mRNA level than non-stressed Tph2+/- mice in amygdala and (D) in the prefrontal cortex. (E) No significant differences were found between groups in GSK-3B expression in hippocampus, (G) in dorsal raphe (I) and in striatum. (F) No significant differences were found between groups in c-fos expression in hippocampus, (H) in dorsal raphe (J) and in striatum. ${ }^{*} \mathrm{p}<0.05$, two-way ANOVA and post hoc Tukey's test. 6-9 animals per group were used. Bars represent Mean \pm SEM.

\section{Predator stress did not alter 5-HT1a and 5-HT2a} expression in wild type and Tph2+/- mice

Rat exposure did not affect significantly 5-HT1a and 5-HT1b expression in the prefrontal cortex (Suppl.Fig.2A,B). For detailed statistical analysis, see main text.

5-HT1a

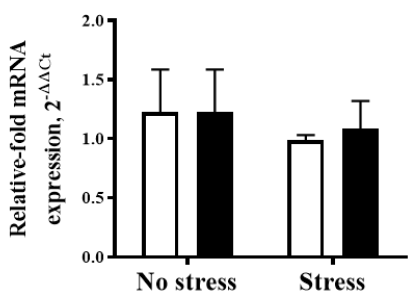

5-HT2a

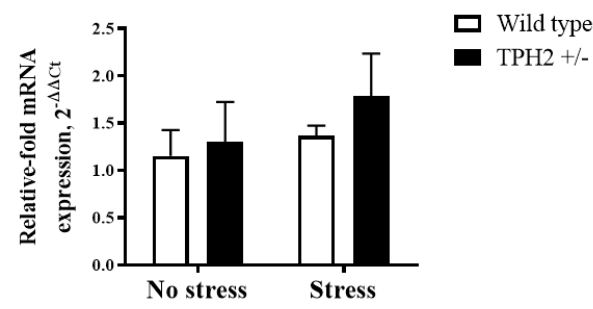

Supplemental Figure 2. Expression of 5-HT1a and 5-HT2a receptors in stressed wild type and Tph2+/- mice. (A) No significant differences were found between groups in 5-HT1a receptor expression and (B) in 5-HT2a receptor expression in the prefrontal cortex. ${ }^{*} \mathrm{p}<0.05$, two-way ANOVA and post hoc Tukey's test. 6-9 animals per group were used. Bars represent Mean \pm SEM. 


\section{Chapter 5}

General discussion 


\section{Chapter 5. General discussion}

5.1. Environmental factors leading to excessive aggression: impact of emotional stress

There are both genetic and environmental contributions towards aggressive behavior (Raine et al., 2002). Studies investigating aggressive behavior within families suggest that $50 \%$ of the variance in aggressive behavior is explained by environmental factors, in both males and females (Tuvblad \& Baker, 2013). Environmental factors that may frequently underlie a predisposition to aggression are poor functioning of the frontal and temporal regions of the brain due to brain injury (Raine, 2004) or birth complications that can result in CNS damage (Liu et al., 2004). Another risk factor for excessive aggression is a deficiency of particular nutrients, including hypoglycemia (Breakey, 1997) and deficiencies in protein (Fishbein \& Pease, 1994), iron (Fishbein, 2001) and zinc (Raine, 1993).

An individual's social environment and the emotional stress associated with it are also critical regulators of neurobiological processes and behavior (Liu, 2004). Emotional stress is a state that is primarily triggered by the perception and cognitive evaluation of adverse events rather than a disturbance of physical nature (Fontes et al., 2014). It is of particular interest in the context of social environments as this may constitute underlying factors that may be amenable to change (Pickett et al., 2003) and most often have a cumulative effect (Stronski et al., 2000). Stress 
results in manifestation of numerous mental disorders (Snijders et al., 2018), e.g., early-life stress (ELS), such as emotional neglect, loss of parents or child abuse, is a major risk factor for the development of anxiety disorders and depression (Heim \& Nemeroff, 2001), as well as excessive aggression and violence in adulthood (Barnow \& Freyberger, 2003). Lower parental attachment, family income, education levels and inadequate social atmosphere serve as risk factors for aggression among adolescents (Dou et al., 2015). Thus, emotional stress is a frequent cause of neuropsychiatric disorders, leading to excessive aggressiveness or depressive disorder (Figure 1).

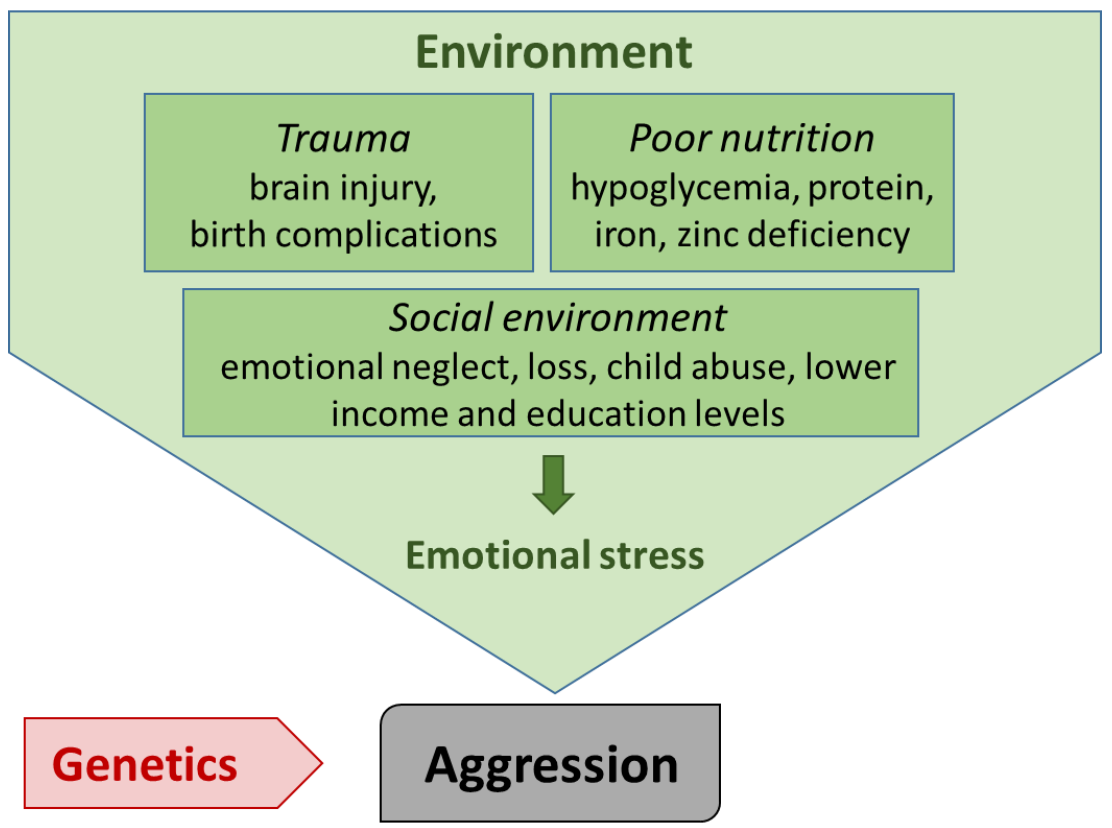

Figure 1. Environmental factors, leading to excessive aggression. Increased aggression can be caused by traumatic brain injury, poor nutrition, and the emotional stress associated with an adverse social environment. 
Appropriate animal models are required to investigate nonphysical sources of emotional stress. However, susceptibility to emotional stress is commonly regarded as a human-specific trait that is challenging to model in other species. Proposed models include maternal separation (Veenema et al., 2006), long-term social isolation (Matsumoto et al., 2002) or exposure to the aggressive behavior of other animals (Suzuki et al., 1998). We have recently reported the development of a new rodent model of emotional stress. In the model of chronic ultrasound exposure, mice are continuously exposed to ultrasounds of variable frequencies chosen to mimic ultrasonic vocalizations of rodents in nature. Sounds in a range of $20-25 \mathrm{kHz}$ correspond to a rodent vocalization of a negative emotional state since they are generated in life-threatening conditions. Frequencies of 25-45 $\mathrm{kHz}$ are associated with a neutral emotional state. The ultrasound is randomly alternated between these frequencies, and rodents are exposed to it for three weeks without any direct physical disturbance (Gorlova et al., 2019; Costa-Nunes et al., in press) (Chapter 2, Chapter 3). Unpredictable alternation of these frequencies is supposed to model the situation of so-called information uncertainty in human, associated with unstable political and economic situations.

Another proposed model is the model of predator stress or rat exposure stress. In this model a mouse is placed in a rat's home cage for five consecutive nights in transparent plastic containers with holes in the lids. These containers protect mice 
from the rats, but allows visual and olfactory contact (Markova et al., 2016, Vignisse et al., 2017). This paradigm is safe and does not pose any physical threat to experimental animal (Strekalova et al., 2005). At the same time, it also reproduces elements of emotional stress based on fear of a predator, since muricide is a natural behavior for rats (Gorlova et al., summitted) (Chapter 4). The presence of predators or their chemical cues is known to have negative effects on the behavioral and neurophysiological states of rodents (Adamec et al., 2004). Both of these models are effective, inexpensive, and most importantly, etiologically relevant. Therefore, they pose useful paradigms for drug research development.

5.2. The role of serotonin, its receptors and Tryptophan hydroxylase-2 in pathological aggression

Alterations in 5-HT neurochemistry have been implicated in the etiology of all major neuropsychiatric disorders, including aggressive behavior (Bortolato et al., 2013). Importantly, low 5HT levels in the brain were shown to be associated with maladaptive forms of excessive aggression and violence rather than with natural defensiveness (de Boer et al., 2009; Lesch \& Merschdorf, 2000).

The synthesis of 5-HT depends on the specific action and rate-limiting step of two isoforms of TPH. While TPH1 is expressed mostly in peripheral tissues, TPH2 is predominant in the CNS. Both clinical and preclinical data indicate that 
aggressive phenotypes are modulated by both TPH isozymes. TPH2 SNPs have shown to be associated with aggression and suicidal behavior (Perez-Rodriguez et al., 2010). E.g., TPH2 SNP rs7305115 was shown to influence suicidal and aggressive behavior (Zhang et al., 2010). Mice, bearing a single-nucleotide mutation $\mathrm{R} 439 \mathrm{H}$ in the TPH2 gene, equivalent to a rare human variant $\mathrm{R} 441 \mathrm{H}$ identified in depressed patients, displayed increased aggression (Beaulieu et al., 2008). The role of different 5-HT receptors in excessive aggression has been extensively studied. In particular, animal studies have documented that 5HT1a receptor agonists attenuate offensive-aggressive responses in rats (Muehlenkamp et al., 1995) and mice (Sanchez et al., 1993), and 5-HT1b knockout mice display impulsive and antagonistic behaviors (Bouwknecht et al., 2001). However, in our study we did not observe significant differences between stressed and non-stressed wild type and Tph2-deficient mice in relative gene expression of 5-HT1a and 5-HT2a receptors (Chapter 4). Conversely, we showed alternations in 5-HT6 receptor in two different models of excessive aggression. Current knowledge about 5-HT6 receptor in the modulation of aggression is still rudimentary (Bortolato et al., 2013). Polymorphisms of the human HTR6 gene were found in association with aggressive behavior in schizophrenia patients (Tsai et al, 1999), but there is almost no evidence about the association of this gene and excessive aggression in experimental animals. Carboline compounds, designed to target AMPA receptors and 5-HT6 
receptors, have been found to have anti-aggressive effects in social stress paradigms in mice (Strekalova et al., 2018). Based on this evidence we investigated whether the expression of these molecules can be considered as possible molecular correlates of increased aggression. In our studies we showed down-regulation of 5-HT6 expression in the ultrasound model of ultrasoundinduced emotional stress (Gorlova et al., 2019) (Chapter 2) and mice heterozygous for the gene encoding Tph2, subjected to rat exposure stress (Gorlova et al., under revision) (Chapter 4).

In general, mice, hypomorphic or deficient in Tph2 were reported to exhibit high aggression, as well as other emotional alterations (Beaulieu et al., 2008). Since mice and humans share many orthologous genes, individual genes identified for behavior can be developed as animal models for human aggression (Lesch $\&$ Merschdorf, 2000). Based on this assumption, mice strains with genetic inactivation of $\mathrm{TPH} 2$ were developed. In the residentintruder paradigm, Tph2-/- males exhibited highly increased aggressive behavior, reflected by decreased latency to the first attack and increased duration of fighting, which was further aggravated by chronic mild stress (Gutknecht et al., 2015; Weidner et al., 2019).

In contrast to Tph2-/- mice, no differences in behavior were detected in Tph2+/- mice, which are characterized by up to $30 \%$ reduction in 5-HT in the raphe (Gutknecht et al. 2012). This indicates that there may be a specific cellular compensatory mechanism during development in Tph2-deficient mice (Waider 
et al. 2013). Most findings have demonstrated that a lack of one TPH2 allele alone is not sufficient to modulate aggression and depression-like behavior and therefore is unlikely to be of physiological significance (Siesser et al., 2010). At the same time, it was suggested that genetic variation in other 5-HT-related genes, restriction or alterations in nutrition, medical treatment and epigenetic modifications acquired during lifespan, may unmask a critical role of TPH2 hypo-expression in the development of pathological symptoms in human (Mosienko et al., 2012) (Figure 2). Since stress can exacerbate brain serotonin deficiency leading to the reduction of the content of neuronal serotonin, we hypothesized that stressed Tph2 heterozygous mice may display increased aggressive behavior, similar to that of null mutants (Gorlova et al., under revision) (Chapter 4). Therefore, male Tph2+/- mice and their wild-type littermates were subjected to a five-day rat exposure predation stress. Stressed mutant mice displayed considerable increases in aggressive- and dominantlike behaviors, whereas stressed wild type mice showed opposing behavioral features, i.e. a reduction of scores of aggression and dominance (Gorlova et al., under revision) (Chapter 4). Concentration of 5-HTP and 5-HIAA were significantly lower in the brain of stressed Tph2+/- mice than in stressed wild type mice (Gorlova et al., under revision) (Chapter 4). These parameters were increased in stressed wild type mice, showing their capacity for increase of serotonin metabolism, which was not demonstrated in Tph2+/- mice (Gorlova et al., under revision) 
(Chapter 4). Thus, partial genetic deficiency of neuronal serotonin synthesis can profoundly alter aggressive behavior under stressful conditions in mice, resulting in a behavioral phenotype that is similar to pro-aggressive effects of a complete deletion of the TPH2 gene. These data suggest that the model of stressed Tph2+/- mice is useful to study the neurobiology of stress-related aggression associated with 5-HT deficiency. This model also enables us to investigate the interaction between partial deficit of TPH2 and stress. Moreover, Tph2+/- mice do not have shorter life span or reduced body size as in Tph2 null mutants, which make them easier to breed in laboratory conditions and to separate behavioral and molecular stress-induced changes from metabolic abnormalities. Finally, since humans do not have a complete lack of a TPH2, the animal model with a partial serotonin deficiency can be considered a more accurate model of stress-induced aggression, associated with serotonin deficiency, representing susceptibility to stress of patients with specific TPH2 polymorphisms. 


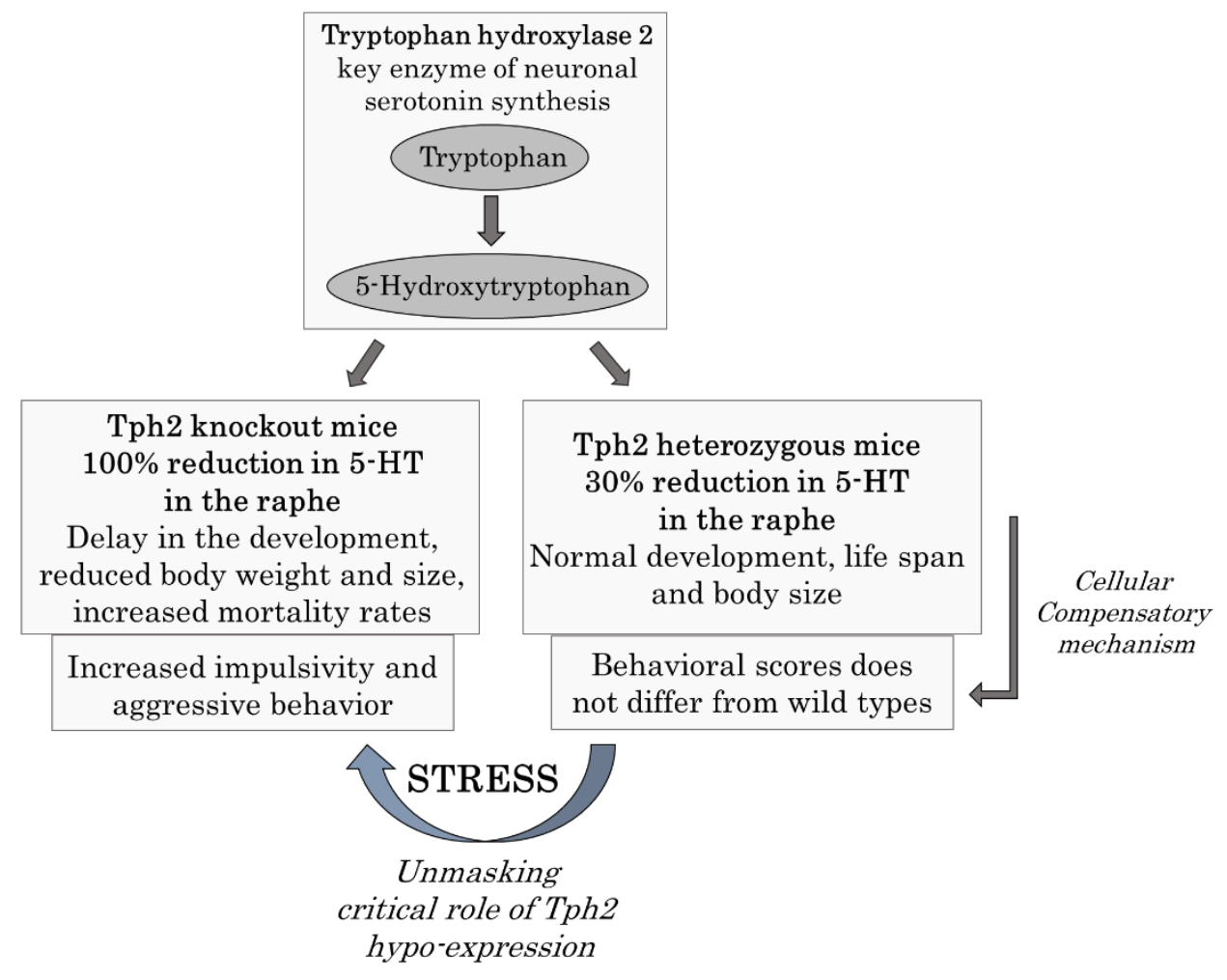

Figure 2. Tph2+/- mice as a model to study the neurobiology of stress-related aggression. Tph2 heterozygous mice display a similar behavioral profile to wild types. Stress results in increased aggression scores, characteristic for unchallenged Tph2 null mutant mice.

\subsection{Altered internalization and expression of AMPA receptor subunits in the mechanisms of neuropsychiatric disorders}

Glutamate is the major excitatory transmitter in the mammalian brain (Takahashi \& Miczek, 2014). Glutamate receptors are widely expressed in brain structures, controlling emotions and behavioral patterns such as agonistic behavior, suggesting their role and, specifically, roles of their subunits, in 
aggression (Davidson et al. 2000). AMPA glutamate receptors, responsible for fast excitatory synaptic transmission in the brain, have been studied more extensively in excessive aggression. Lower levels of agonistic behavior, manifested in reduced duration and frequency, increased latency to attack and decreased overall number of mice that displayed aggression, were found in GluA1-deficient mice (Vekovisheva et al., 2004) and GluA3-deficient mice (Adamczyk et al., 2012). At the same time, aggression towards the intruder was found to be absent in male mice lacking the AMPA receptor subunit GluA2 (Adamczyk et al., 2012).

In our study, chronic ultrasound exposure resulted in reduced levels of GluA1 and GluA3 expression in prefrontal cortex and hippocampus, and the levels of cell surface expression of these subunits was decreased in the dentate gyrus (Gorlova et al., 2019; Costa-Nunes et al., in press) (Chapter 2, Chapter 3). Expression of GluA2 was, in contrast, increased, but the cell surface expression of the protein decreased. This probably reflects increased turnover of the protein in neuronal populations, which may have resulted in an increased demand for transcript. However, the discrepancy may also reflect increased expression in GluA2 expression in non-neuronal cell populations. The GluA2 subunits of the AMPA receptors, also expressed in astrocytes, are crucial determinants in controlling calcium permeability and function (Fan et al., 1999). However, while GluA3 is increased in reactive astrocytes GluA2 is typically unchanged (Newcombe et 
al., 2008). Concerning the differences that we observed between the regulation of subunit expression reported here in our study, several studies have suggested that these receptor subunits may play distinct roles in the regulation of AMPA receptor trafficking and synaptic plasticity. As described above, GluA1/GluA3- and GluA2- knockout mice displayed opposite changes in aggressive behavior. Thus, it is possible that its expression can be changed differently, depending on environmental conditions. GluA2 incorporation into AMPA receptors seems to be particularly important in synaptic scaling, and, thus, changes in the ratios of GluA1 and GluA2 suggest that synaptic plasticity is likely to be altered, which was also shown in the current study.

Despite the difference in the expression of different AMPA subunits, all acted similarly in terms of internalization, showing increased internalization index (Costa-Nunes et al., in press) (Chapter 3). Internalization is a complex regulatory process, which terminates receptor cell surface signaling and designates activated receptors for degradation (Figure 3). It plays a key role in the modulation of receptor bioavailability, and thus, downstream signaling. Ligand-induced receptor internalization serves as an important modulator of receptor signaling (Pradhan et al., 2016). Endocytosis also represents an important mechanism regulating cell-surface expression of neurotransmitter receptors in neurons. This internalization regulates cell-surface expression of AMPA receptor in neurons (van Rijnsoever et al., 2005). The subcellular localization of 
AMPA receptors indicates that $60-70 \%$ of the receptors are at intracellular sites, thus forming a reserve pool of receptors unless they possess an unknown duty distinct from the silent synapse theory (Lee et al. 2001). In our study we observed an increase of internalized GluA1-3 puncta, while number of surface puncta was, in contrary, decreased (Costa-Nunes et al., in press) (Chapter 3). Observed increase of the internalization process, defined as a ratio of internalized / surface AMPA receptor subunits, may suggest stress-induced decrease in glutamate-mediated neurotransmission that is realized via GluA1-3, which may dampen negative effects of glutamatergic excitotoxicity, an important pathogenetic factor of stress (Caudal et al., 2010; Bonini et al., 2016). Reported increase in the mRNA levels of GluA2 may indicate compensatory changes in this regulation; a lack of similar alternations in gene expression of GluA1 and GluA3 subunits could presumably be due to distinct temporal features of these compensatory processes of balancing the ratio of internalization/gene expression of AMPA receptor subunits, since these processes are interrelated (Park et al., 2009). Thus, present findings suggest a suppressive effect of ultrasound stress on the AMPA receptor-mediated signaling and synaptic plasticity that can underlie stress-related emotional abnormalities (Gorlova et al., 2019; Costa-Nunes et al., in press) (Chapter 2, Chapter 3). 


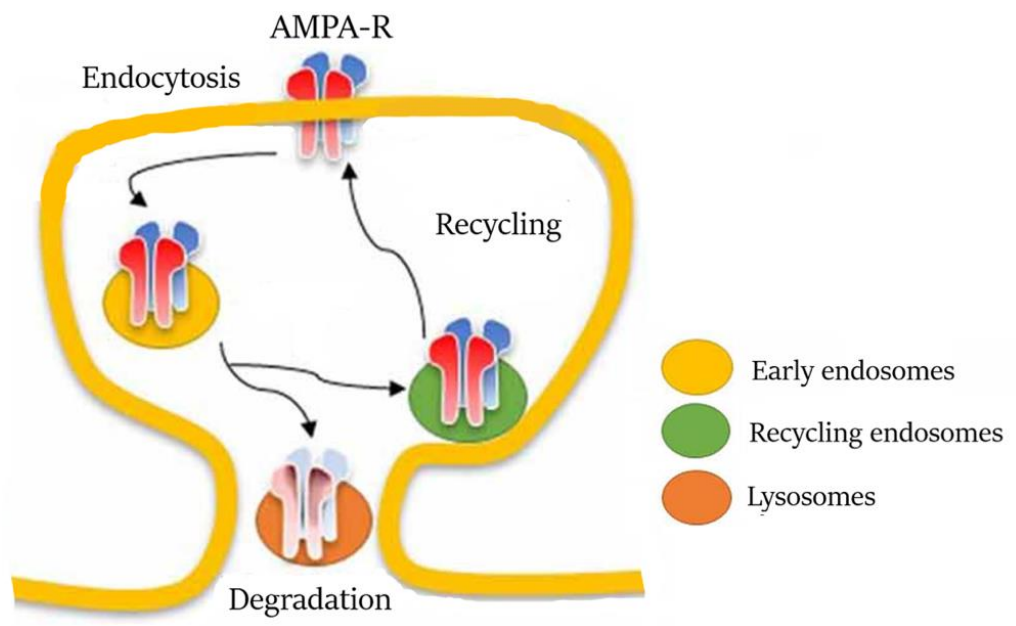

Figure 3. Scheme of AMPA receptors (AMPA-Rs) endocytosis. Enhanced internalization and retention of AMPA-Rs as the mechanism of negative regulation of its surface levels. AMPA-Rs are constitutively endocytosed and sorted between lysosomes for degradation or recycling endosomes for recycling back to the plasma membrane (adapted from Morretto \& Passafaro, 2018).

5.4. Interplay between oxidative stress, neuroinflammation and altered neuroplasticity in excessive aggression, depressive-like and anxiety-like behavior: evidence from clinical data

Inflammation is a normal protective response to infection or tissue injury. However, an uncontrolled inflammatory response, which can be caused by exposure to stress of various origins, results in extensive cell and tissue damage, leading to the impairment of blood-brain barrier (BBB) (Menard et al., 2017). In the current study we tested the hypothesis that markers of 
inflammation would show a prolonged increase in our new model of emotional stress.

Human studies showed that individuals with excessive aggression display increased inflammatory cytokine levels and dysregulated immune responses such as slower wound healing. Pathological levels of aggression in intermittent explosive disorder or psychosis appeared to be linked to heightened levels of proinflammatory cytokines (Coccaro et al., 2014). Similar findings have been observed in patients with depression, and comorbidity of depression and aggression was correlated with stronger immune dysregulation. Therefore, dysregulation of the immune system associated with prolonged social stress may be one of the mechanisms leading to greater aggression and depression (Takahashi et al., 2018). These data suggest specific disruptions within the immune system which correlate with a common endophenotype for both depression and anger/aggression in humans. This complex of behavioral alternations was observed in mice subjected to chronic ultrasound stress, that was shown to induce excessive aggression, associated with depressive-like and anxiety-like behavior (Gorlova et al., 2019; Costa-Nunes et al., in press) (Chapter 2, Chapter 3), and we expected significant changes in pro-inflammatory cytokines in stressed mice. Human, individuals with excessive aggression often display higher level of IL-6 and TNF than non-aggressive individuals. Interestingly, in both male and female individuals with excessive aggression, there were higher levels of plasma IL-6, only when they concurrently 
suffered depressive syndrome (Suarez, 2003). This correlation was absent among individuals without depressive traits (Stewart et al., 2008). In our study we showed that chronic variable ultrasound exposure increased levels of TNF, IL-1B and IL-6, associated with increased aggression, as well as a depressive-like state. This was in line with the above-described correlation between IL- 6 and the manifestation of depressive-like behavioral traits, since ultrasound-exposed mice demonstrated despair behavior in the forced swim test.

Numerous studies have also shown that aggressive animals have increased levels of several anti-inflammatory cytokines, such as IL-4 and IL-10 (Mommersteeg et al., 2008). Unlike humans, aggressive animals are supposed to be more protected from infections and have faster wound healing than animals with low aggression (Kiecolt-Glaser et al., 2005). However, it is hypothesized that activation of the immune system, adaptive in dominant individuals, can become maladaptive in extreme cases of pathological aggression, which make up most of the cases in human studies. Thus, dominant individuals with normal aggression level may have well-balanced activation of both proinflammatory and anti-inflammatory cytokines to actively cope with possible injury, caused by aggressive interactions. In contrast, pathological aggression, especially with a comorbid depression-like phenotype, may induce dysregulation of the immune system leading to a pro-inflammatory state in the animal (Takahashi et al., 2018). In the current work we observed no 
differences between groups in the expression of antiinflammatory cytokine IL-10 (Costa-Nunes et al., in press) (Chapter 3). Thus, we suppose that this exact case of maladaptive aggression was observed in the model of ultrasound stress, where mice demonstrate not just altered level of aggression, but also depressive-like changes and profoundly increased anxiety-like behavior, observed in o-maze and open field tests (Gorlova et al., 2019; Costa-Nunes et al., in press) (Chapter 2, Chapter 3). This fact once again allows us to suggest a remarkable similarity of ultrasound-induced behavioral and molecular changes to the clinical picture of excessive aggression in human.

Apart from an exacerbated inflammatory response, we also showed activations of GSK-3B in the brain induced by emotional stress (Costa-Nunes et al., in press) (Chapter 3), GSK-3 B is considered as a molecular hub of distress (Beurel et al., 2015) and is a well-established physiological mediator of elevated synthesis of cytokines, including IL-1B and TNF (Green \& Nolan, 2012). Remarkably, GSK-3B is known to inhibit production of the antiinflammatory cytokine IL-10 (Jope et al., 2017), which, as mentioned above, was not increased in this model. The physiological antagonist of GSK-3B, Akt (Manning \& Toker, 2017), was unaltered in our study (Costa-Nunes et al., in press) (Chapter 3). Elevated GSK-3B activities were shown to alter glutamatergic neurotransmission including AMPA-mediated transmission (Wei et al., 2010), which was affected in the current study, as described above. Moreover, upregulation of GSK-3B 
results in increases of oxidative stress markers (Potz et al., 2018), and suppression of hippocampal plasticity (Peineau at al., 2008). Both of these stress-related processes were induced by chronic ultrasound exposure, which is described below.

Concentration of oxidative molecules such as reactive oxygen species (ROS) and reactive nitrogen species (RNS) are known to be increased by inflammation, as well as stress and emotional disturbance (Tsaluchidu et al., 2008). Recent studies have shown that numerous psychiatric disorders, such as depression and anxiety, result in increased reactive oxygen generation (Arranz et al., 2007). Since oxidative stress is an inherent part of inflammation, and inflammation is associated with behavioral aggression both in lower mammals and humans, markers of oxidative stress were hypothesized by numerous researches to be related to aggression in human subjects (Coccaro et al., 2016). Inflammatory cells secrete a large number of cytokines and chemokines responsible for the production of ROS (Sánchez et al., 2015), with ROS in turn stimulating the production of inflammatory cytokines such as IL-1B and TNF (Naik et al., 2011). We hypothesized that increases in oxidative stress play a major role in the development of excessive expression and other behavioral alternations in the new model of emotional stress. Ultrasound-exposed mice showed stressinduced increase of protein carbonyls, a marker of protein oxidation, malondialdehyde, a marker of lipid peroxidation, as well as increased content of total glutathione, the main 
intracellular thiol that plays crucial role in defending the cell against ROS (Gorlova et al., 2019; Costa-Nunes et al., in press) (Chapter 2, Chapter 3). It is important to mention that most of the investigated behavioral and molecular parameters correlated with hippocampal malondialdehyde content (Costa-Nunes et al., in press) (Chapter 3). However, these correlations were only present in stressed, but not in non-stressed mice which further highlights the role of oxidative stress in neurobiological abnormalities associated with emotional stress.

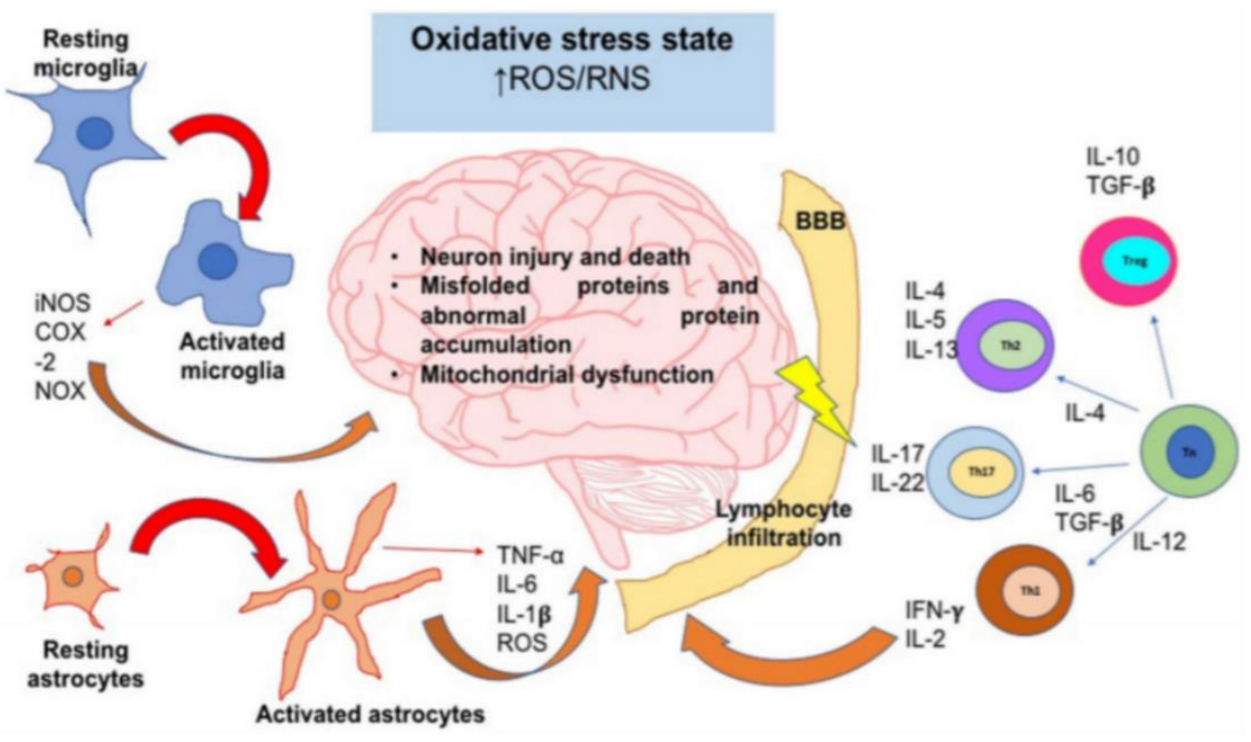

Figure 4. Oxidative stress state enhances neuroinflammation and neurodegeneration. In an oxidative stress state, ROS and RNS levels are increased; these reactive species can activate signaling pathways that lead to the activation of the major glial inflammatory characters: microglia and astrocytes. Glial cells secrete proinflammatory factors which positively feedback the neuroinflammatory response. On the other hand, secreted factors and peripheral cytokines can disrupt the BBB integrity, and T cells are able to infiltrate into CNS, that results in the positive feedback of 
neuroinflammation. Inflammatory cells and secreted factors lead to neurodegeneration, in which the most characteristic feature is neuronal injury and death. iNOS is inducible nitric oxide synthase; COX-2 is cyclooxigenase-2; NOX is NADPH oxidase; Th is T helper cell; Tn is T naive cell; Treg is T regulatory cell; TGF- $B$ is transforming growth factor beta (adapted from Solleiro-Villavicencio \& Rivas-Arancibia, 2018).

Cytokines have also been shown to decrease the neurotrophic support and to reduce neurogenesis in several brain areas, particularly in the hippocampus (Hashmi et al., 2013), which, in turn, may contribute to the reduction of neuronal plasticity and, as a result, to aggression- and depression-related dysfunction. Impaired neurogenesis in the hippocampus and atrophy of dendritic trees after chronic stress was shown to be associated with increased anxiety and aggression (Bloss et al., 2010). In the current study we also demonstrated impaired neurogenesis, manifested in decreased number of Ki67-positive cells, a marker of cell proliferation, in the dentate gyrus of ultrasound-stressed mice (Costa-Nunes et al., in press) (Chapter 3). In addition, we explored a broader spectrum of plasticity markers, that were less studied in terms of aggression. In particular, we selected B-catenin because it is significantly downregulated in a subgroup of mice that are susceptible to stress-induced anhedonia in a chronic stress model, associated with elevated aggression (Strekalova et al., 2011). Postsynaptic density protein 95 (PSD95) was chosen for its role as a scaffolding protein that regulates synaptic formation, and because it was recently reported that decreased levels of PSD95 are associated 
with increased aggression in rodent models (Been et al., 2016; Winkler et al., 2017). Furthermore, PSD95 is known for its role for AMPA receptor internalization (Kim et al., 2007), observed in our study (Gorlova et al., 2019; Costa-Nunes et al., in press) (Chapter 2, Chapter 3), therefore, alternations in the AMPA subunits internalization index may be associated with altered expression of PSD95 (Gorlova et al., 2019) (Chapter 2). Finally, decreased expression of the neuronal cell adhesion molecule NCAM, a well-established plasticity factor, and its functionally important polysialylated form PSA-NCAM, have also been found to be related to increased levels of aggression and territoriality in mice (Albrecht \& Stork, 2012). The expression of all these plasticity factors was decreased after ultrasound stress, suggesting role of these molecules in the development of stressinduced aggression, depressive-like and anxiety-like behavior.

\subsection{Antioxidant compounds as potential treatment of} excessive aggression and associated conditions: perspectives of clinical use of thiamine compounds and herbal treatments

As stress-induced behavioral changes, including aggression, depression and anxiety, are associated with oxidative stress and inflammation-related processes in the brain, we hypothesized that compounds with antioxidant and anti-inflammatory properties can counteract negative effects of the stress. It was shown that antidepressants, such as imipramine and fluoxetine, 
have anti-inflammatory effects and reduce the production of the pro-inflammatory cytokines, while increasing production of the anti-inflammatory cytokine IL-10 (Köhler et al., 2018). Additionally, Vitamin $\mathrm{E}$ and beta-carotene were shown to be effective to prevent all aggression parameters in acute and chronic studies against L-DOPA induced aggression (Hira et al., 2017).

Another vitamin that, according to our suggestion, can be used for management of excessive aggression, is thiamine (vitamin B1), an essential nutrient crucial for normal growth and development of the organism (Liu et al., 2017), that plays a key role in cell metabolism (Osiezagha et al., 2013) and is able to cross the BBB (Greenwood et al., 1982). Thiamine deficiency (TD) causes impairment of oxidative metabolism and neurodegeneration, which are observed in various diseases, including aggression, depression and anxiety. TD also leads to an immunological imbalance, characterized by increased proinflammatory cytokines, resulting in neuroinflammation (Terlecky et al., 2012). These data suggest that thiamine may act as an antioxidant and anti-inflammatory compound, and in numerous studies it was shown to decrease ROS production (Huang et al., 2010). For this reason, we chose thiamine as a new pharmacological way for the management of excessive aggression, induced by ultrasound stress, which was shown to be associated with oxidative stress and inflammation (Gorlova et al., 2019; Costa-Nunes et al., in press) (Chapter 2, Chapter 3). We also 
tested benfotiamine, a synthetic thiamine precursor, which dephosphorylation product S-benzoylthiamine has high lipid solubility, that facilitates permeation in cell membranes (Sambon et al., 2019). As a result, it allows for higher levels of thiamine in the brain, and thus we expected it to be more effective than thiamine in preventing stress-induced aggression and other behavioral and molecular changes.

Altogether, we demonstrated the effectiveness of both thiamine and benfotiamine in preventing stress-induced aggression and depressive-like state, as well as neuroinflammation, oxidative stress and altered neuroplasticity. Both compounds also reversed ultrasound-induced changes in expression of AMPA receptor subunits and 5-HT6 receptor, and benfotiamine was indeed more effective in precluding the alternations in some of these parameters, which is in line with the notion of its higher bioavailability (Gorlova et al., 2019) (Chapter 2). Thus, thiamine and its precursor benfotiamine can be proposed as new ways of pharmacologically managing excessive aggression. It is important to mention that existing pharmaceuticals are known for their numerous side effects, whereas thiamine is well tolerated even at high doses (Bettendorff \& Wins, 2013).

Another approach for the treatment of stress-related conditions with minimum side effects could be herbal medicines, and is increasingly being considered an important complement to modern medicine. Establishing its validity in the treatment for 
various diseases is crucial to increase its acceptability. Numerous plants or specific combinations of herbs have demonstrated a potential role in the inhibition of proinflammatory cytokines and radical-scavenging capacities (Ghasemian et al., 2016).

In our study, chronic treatment of mice with a herbal composition, containing seaweed, vanilla, ginger, lemon, orange, elderberries and other elements, normalized evaluated behavioral parameters of anxiety-like and aggressive behaviors, and prevented the increase in malondialdehyde content and overexpression of pro-inflammatory cytokines in ultrasound-exposed mice (Costa-Nunes et al., in press) (Chapter 3). The number of elements in the herbal composition can be considered as a limitation, since we cannot suggest what exact herb or combination contributed significantly. However, current results demonstrate beneficial effects of this herbal composition that can most likely be explained by its anti-oxidant and antiinflammatory properties.

Thus, administration of thiamine, benfotiamine and herbs counteract aggression and other behavioral effects of emotional stress presumably by antagonizing oxidative and inflammatory processes, further emphasizing the role of oxidative stress and neuroinflammation in neuropsychiatric disorders, such as excessive aggression, depression and anxiety. Currently we are demonstrating the therapeutic potential of these compounds and suggest them as a promising solution for management of stressrelated emotional disturbances. 


\section{References}

Adamczyk A., Mejias R., Takamiya K., Yocum J., Krasnova I., Calderon J., Cadet J.L., Huganir R., Pletnikov M., Wang T. GluA3deficiency in Mice is Associated with Increased Social and Aggressive Behavior and Elevated Dopamine in Striatum. Behav Brain Res. 2012, 229: 265-272.

Adamec R., Walling S., Burton P. Long-lasting, selective, anxiogenic effects of feline predator stress in mice. Physiol. Behav. 2004, 83: 401-410.

Albrecht A., Stork O. Are NCAM deficient mice an animal model for schizophrenia? Front Behav Neurosci. 2012, 6: 43.

Arranz L., Guayerbas N., De la Fuente M. Impairment of several immune functions in anxious women. J Psychosom Res. 2007, 62: $1-8$.

Barnow S., Freyberger H. J. The family environment in early life and aggressive behavoir in adolescents and young adults. Neurobiology of Aggression: Understanding and Preventing Violence, 2003: 213-229.

Beaulieu J.M., Zhang X., Rodriguiz R.M., Sotnikova T.D., Cools M.J., Wetsel W.C., Gainetdinov R.R., Caron M.G. Role of GSK3 beta in behavioral abnormalities induced by serotonin deficiency. Proc Natl Acad Sci USA. 2008, 105: 1333-1338.

Been L.E., Moore K.M., Kennedy B.C., Meisel R.L., 2016. Metabotropic Gglutamate rReceptor and Ffragile X Ssignaling in a Ffemale mModel of eEscalated Aaggression. Biol Psychiatry. 79, 685-692. doi: 10.1016/j.biopsych.2015.07.021.

Bettendorff L., Wins P. Biological functions of thiamine derivatives: Focus on non-coenzyme roles, OA Biochem. 2013, 1.

Beurel E., Grieco S.F., Jope R.S. Glycogen synthase kinase-3 (GSK3): regulation, actions, and diseases. Pharmacol Ther. 2015, 148: 114-131.

Bloss E.B., Janssen W.G., McEwen B.S., Morrison J.H. Interactive effects of stress and aging on structural plasticity in the prefrontal cortex. The Journal of Neuroscience. 2010, 30: 67266731.

Bonini D., Mora C., Tornese P., Sala N., Filippini A., La Via L., Milanese M., Calza S., Bonanno G., Racagni G., Gennarelli M., Popoli M., Musazzi L., Barbon A. Acute Footshock Stress Induces TimeDependent Modifications of AMPA/NMDA Protein Expression and AMPA Phosphorylation. Neural Plast. 2016: 7267865. 
Bortolato M., Pivac N., Seler D.M., Perkovic M.N., Pessia M., Giovanni G. The role of serotonergic system at the interface of aggression and suicide. Neuroscience 2013, 236: 160-185.

Bouwknecht J.A., Hijzen T.H., van der Gugten J., Maes R.A., Hen R., Olivier B. Absence of 5-HT(1B) receptors is associated with impaired impulse control in male 5-HT(1B) knockout mice. Biol Psychiatry. 2001, 49: 557-568.

Breakey I. The role of diet and behavior in childhood. Journal of Pediatric Child Health. 1997, 33: 190-194.

Caudal D., Godsil B.P., Mailliet F., Bergerot D., Jay T.M. Acute Stress Induces Contrasting Changes in AMPA Receptor Subunit Phosphorylation within the Prefrontal Cortex, Amygdala and Hippocampus. PLoS One. 2010; 5: e15282.

Coccaro E.F., Lee R., Coussons-Read M. Elevated plasma inflammatory markers in individuals with intermittent explosive disorder and correlation with aggression in humans. JAMA Psychiatry. 2014, 71: 158.

Coccaro E.F., Lee R., Gozal D. Elevated Plasma Oxidative stress markers in individuals with intermittent explosive disorder and correlation with aggression in humans. Biol Psychiatry. 2016, 79: 127-135.

Costa-Nunes J.P., Gorlova A., Pavlov D., Cespuglio R., Gorovaya A., Proshin A., Umriukhin A., Ponomarev E., Kalueff A., Strekalova T., Schroeter C., 2020. Ultrasound stress compromises the correlates of emotional-like states and brain AMPAR expression in mice: effects of antioxidant and anti-inflammatory herbal treatment. Stress. (in press)

Davidson R.J., Jackson D.C., Kalin, N.H. Emotion, plasticity, context, and regulation: perspectives from affective neuroscience. Psychol Bull. 2002, 126: 890-909.

de Boer S.F., Caramaschi D., Natarajan D., Koolhaas J.M. The vicious cycle towards violence: focus on the negative feedback mechanisms of brain serotonin neurotransmission. Front Behav Neurosci. 2009, 3: 52.

Dou C., Wei Z., Jin K., Wang H., Wang X., Peng Z. Family and social environmental factors associated with aggression among Chinese adolescents. Sch Psychol Q. 2015, 30: 421-430.

Fan D., Grooms S.Y., Araneda R.C., Johnson A.B., Dobrenis K., Kessler J.A., Zukin R.S., 1999. AMPA receptor protein expression and function in astrocytes cultured from hippocampus. J Neurosci Res. 57, 557-571. 
Fishbein D.H. Biobehavioral perspective in criminology. The Wadsworth series in criminological theory. Wadsworth/Thomson Learing; Belmont, CA: 2001.

Fishbein D.H., Pease S.E. Diet, nutrition, and aggression. Journal of Offender Rehabilitation. 1994, 21: 117-144.

Fontes M.A., Xavier C.H., Marins F.R., Limborço-Filho M., Vaz G.C., Müller-Ribeiro F.C., Nalivaiko E. Emotional stress and sympathetic activity: Contribution of dorsomedial hypothalamus to cardiac arrhythmias. Brain Research. 2014, 1554: 49-58.

Ghasemian M., Owlia S., Owlia M.B. Review of Anti-Inflammatory Herbal Medicines. Adv Pharmacol Sci. 2016: 9130979.

Gorlova A., Pavlov D., Anthony D.C., Ponomarev E., Proshin A., Sambon M., Schmitt-Boeher A., Lesch K.P., Bettendorff L., Strekalova T. Thiamine and benfotiamine counteract ultrasound-induced aggression, normalize AMPA receptor expression and plasticity markers, and reduce oxidative stress in mice. Neuropharmacology, 2019, 156: 107543

Gorlova A, Ortega G, Waider J, Bazhenova N, Veniaminova E, Proshin A, Kalueff A, Anthony DC, Lesch KP, Strekalova T. Stressinduced aggression in heterozygous TPH2 mutant mice is associated with alterations in serotonin metabolism and expression of 5-HT6 and AMPA subunit 2A receptors. Journal of Affective Disorders (under revision).

Green H.F., Nolan Y.M. GSK-3 mediates the release of IL-1B, TNF-a and IL-10 from cortical glia. Neurochem Int. 2012; 61: 666-671.

Greenwood J., Love E.R., Pratt O.E. Kinetics of thiamine transport across the blood-brain barrier in the rat. J Physiol. 1982, 327: 95-103.

Gutknecht L., Araragi N., Merker S., Waider J., Sommerlandt F.M., Mlinar B., Baccini G., Mayer U., Proft F., Hamon M., Schmitt A.G., Corradetti R., Lanfumey L., Lesch K.P. Impacts of brain serotonin deficiency following Tph2 inactivation on development and raphe neuron serotonergic specification. PLoS One. 2012, 7 :e43157.

Gutknecht L., Popp S., Waider J., Sommerlandt F.M.J., Göppner C., Post A., Aeif R., van den Hove D., Strekalova T., Schmitt A., Colaso M.B.N., Sommer C., Palme R., Lesch K.P. Interaction of brain 5-HT synthesis deficiency, chronic stress and sex differentially impact emotional behavior in Tph2 knockout mice. Psychopharmacology (Berl). 2015, 232: 2429-2441. 
Hashmi A.M., Butt Z., Umair M. Is depression an inflammatory condition? A review of available evidence. J. Pak. Med. Assoc. 2003, 63: 899-906.

Heim C., Nemeroff C. B. The role of childhood trauma in the neurobiology of mood and anxiety disorders: preclinical and clinical studies. Biol. Psychiatry. 2001, 49: 1023-1039.

Hira S., Saleem U., Anwar F., Ahmad B. Antioxidants Attenuate Isolation- and L-DOPA-Induced Aggression in Mice. Front Pharmacol. 2017, 8: 945.

Huang H.M., Chen H.L., Gibson G.E. Thiamine and oxidants interact to modify cellular calcium stores. Neurochem Res. 2010, 35: 2107-2116.

Jope R.S., Cheng Y., Lowell J., Worthen R.J., Sitbon Y.H., Beurel E. Stressed and inflamed, can GSK3 be blamed? Trends Biochem Sci. 2017; 42: 180-192.

Kiecolt-Glaser J.K., Loving T.J., Stowell J.R., Malarkey W.B., Lemeshow S., Dickinson S. L., Glaser R. Hostile marital interactions, proinflammatory cytokine production, and wound healing. Arch. Gen. Psychiatry. 2005, 62: 1377.

Kim M.J., Futai K., Jo J., Hayashi Y., Cho K., Sheng M. Synaptic accumulation of PSD-95 and synaptic function regulated by phosphorylation of serine-295 of PSD-95. Neuron. 2007, 56: 488502.

Köhler C.A., Freitas T.H., Stubbs B., Maes M., Solmi M., Veronese N., de Andrade N.Q., Morris G., Fernandes B.S., Brunoni A.R., Herrmann N., Raison C.L., Miller B.J., Lanctôt K.L., Carvalho A.F. Peripheral alterations in cytokine and chemokine levels after antidepressant drug treatment for major depressive disorder: systematic review and meta-analysis. Mol. Neurobiol. 2018, 55: 4195-4206.

Lee S.H., Valtschanoff J.G., Kharazia V.N., Weinberg R., Sheng M. Biochemical and morphological characterization of an intracellular membrane compartment containing AMPA receptors. Neuropharmacology. 2001; 41: 680-692.

Lesch K.P., Merschdorf U. Impulsivity, aggression, and serotonin: a molecular psychobiological perspective. Behav Sci Law. 2000, 18: 581-604.

Linnoila M., Virkkunen M., Cheinin M., Nuutila A., Rimon R, Goodwin F.K. Low cerebrospinal fluid 5-hydroxindoleacetic acid concentration differentiates impulsive from nonimpulsive violent behavior. Life Sci. 1983, 33: 2609-2614. 
Liu D., Ke Z., Luo J. Thiamine Deficiency and Neurodegeneration: The Interplay among oxidative stress, endoplasmic reticulum stress and autophagy. Mol Neurobiol. 2017, 54: 5440-5448.

Liu J.H. Prenatal \& perinatal complications as predispositions to externalizing behavior. Journal of Prenatal \& Perinatal Psychology \& Health. 2004, 18: 301-311.

Manning B.D., Toker A. AKT/PKB Signaling: Navigating the Network. Cell. 2017. 169: 381-405.

Markova N., Bazhenova N., Anthony D.C., Vignisse J., Svistunov A., Lesch K.P., Bettendorff L., Strekalova T. Thiamine and benfotiamine improve cognition and ameliorate GSK-38associated stress-induced behaviours in mice. Prog Neuropsychopharmacol Biol Psychiatry. 2016, 75: 148-156.

Matsumoto K., Pinna G., Puia G., Guidotti A., Costa E. Social isolation stress-induced aggression in mice: a model to study the pharmacology of neurosteroidogenesis. Stress. 2005, 8: 85-93.

Menard C., Pfau M. L., Hodes G. E., Kana V., Wang V. X., Bouchard S., Takahashi A., Flanigan M.E., Aleyasin H., LeClair K.B., Janssen W.G., Labonté B., Parise E.M., Lorsch Z.S., Golden S.A., Heshmati M., Tamminga C., Turecki G., Campbell M., Fayad Z.A., Tang C.Y., Merad M., Russo S.J. Social stress induces neurovascular pathology promoting depression. Nat. Neurosci. 2-17. 20, 1752-1760.

Mommersteeg P.M.C., Vermetten E., Kavelaars A., Geuze E., Heijnen C.J. Hostility is related to clusters of T-cell cytokines and chemokines in healthy men. Psychoneuroendocrinology. 2008, 33. 1041-1050.

Moretto E., Passafaro M. Recent Findings on AMPA Receptor Recycling. Front Cell Neurosci. 2018, 12: 286.

Mosienko V., Bert B., Beis D., Matthes S., Fink H., Bader M., Alenina N. Exaggerated aggression and decreased anxiety in mice deficient in brain serotonin. Transl Psychiatry. 2012, 2: e122.

Muehlenkamp F., Lucion A., Vogel W.H. Effects of selective serotonergic agonists on aggressive behavior in rats. Pharmacol Biochem Behav. 1995, 50: 671-674.

Naik E., Dixit V.M. Mitochondrial reactive oxygen species drive proinflammatory cytokine production. J. Exp. Med. 2011, 208: 417420.

Newcombe J., Uddin A., Dove R., Patel B., Turski L., Nishizawa Y., Smith T., 2008. Glutamate receptor expression in multiple sclerosis lesions. Brain Pathol. 18, 52-61. 
Osiezagha K., Ali S., Freeman C., Barker N.C., Jabeen S., Maitra S., Olagbemiro Y., Richie W., Bailey R.K. Thiamine deficiency and delirium. Innov Clin Neurosci. 2013, 10: 26-32.

Park J., Voitenko N., Petralia R.S., Guan X., Xu J., Steinberg J.P., Takamiya K., Sotnik A., Kopach O., Huganir R.L., Taocorresponding Y. Persistent Inflammation Induces GluR2 Internalization via NMDA Receptor-Triggered PKC Activation in Dorsal Horn Neurons. J Neurosci. 2009; 29: 3206-3219.

Peineau S., Bradley C., Taghibiglou C., Doherty A., Bortolotto Z.A., Wang Y.T., Collingridge G.L. The role of GSK-3 in synaptic plasticity. Br J Pharmacol. 2008, 153: S428-S437.

Perez-Rodriguez M.M., Weinstein S., New A.S., Bevilacqua L., Yuan Q., Zhou Z., Hodgkinson C., Goodman M., Koenigsberg H.W., Goldman D., Siever L.J. Tryptophan-hydroxylase 2 haplotype association with borderline personality disorder and aggression in a sample of patients with personality disorders and healthy controls. J Psychiatr Res. 2010, 44: 1075-1081.

Pickett W., Simons-Morton B., Dostaler S., Iannotti R.J. Social environments and physical aggression among 21,107 students in the United States and Canada. J Sch Health. 2009, 79: 160-168.

Potz B.A., Scrimgeour L.A., Sabe S.A., Clements R.T., Sodha N.R., Sellke F.W. Glycogen Synthase Kinase 36 Inhibition Reduces Mitochondrial Oxidative Stress in Chronic Myocardial Isc Peineau S., Bradley C., Taghibiglou C., Doherty A., Bortolotto Z.A., Wang Y.T., Collingridge G.L. The role of GSK-3 in synaptic plasticity. Br J Pharmacol. 2008; 153(Suppl 1): S428S437.hemia. J Thorac Cardiovasc Surg. 2018; 155: 2492-2503.

Pradhan A.A., Tawfik V.L., Laboy A.F., Scherrer G. In Vivo Techniques to Investigate the Internalization Profile of Opioid Receptors. Methods Mol Biol. 2015, 1230: 87-104.

Raine A. Annotation: The role of prefrontal deficits, low autonomic arousal and early health factors in the development of antisocial and aggressive behavior in children. Journal of Child Psychology \& Psychiatry \& Allied Disciplines. 2002, 43: 417-434.

Raine A. The psychopathology of crime: Criminal behavior as a clinical disorder. Academic; San Diego: 1993.

Raine A., Ishikawa S.S., Arce E., Lencz T., Knuth K.H., Bihrle S., LaCasse L., Colletti P. Hippocampal structural asymmetry in unsuccessful psychopaths. Biol Psychiatry. 2004, 55: 185-191.

Sambon M., Napp A., Demelenne A., Vignisse J., Wins P., Fillet M., Bettendorff L. Thiamine and benfotiamine protect 
neuroblastoma cells against paraquat and B-amyloid toxicity by a coenzyme-independent mechanism. Heliyon. 2019, 5: e01710.

Sánchez A., Calpena A.C., Clares C. Evaluating the Oxidative Stress in

Inflammation: Role of Melatonin. Int J Mol Sci. 2015, 16: 1698117004.

Sanchez C., Arnt J., Hyttel J., Moltzen E.K. The role of serotonergic mechanisms in inhibition of isolation-induced aggression in male mice. Psychopharmacology. 1993, 110: 53-59.

Snijders C., Pries L.P., Sgammeglia N., Al Jowf G., Youssef N.A., de Nijs L., Guloksuz S., Rutten B.P.F., 2018. Resilience Against

Traumatic Stress: Current Developments and Future Directions. Front Psychiatry. 9, 676.

Siesser W.B., Zhang X., Jacobsen J.P., Sotnikova T.D., Gainetdinov R.R., Caron M.G. Tryptophan hydroxylase 2 genotype determines brain serotonin synthesis but not tissue content in C57Bl/6 and BALB/c congenic mice. Neurosci Lett. 2010, 481: 611.

Solleiro-Villavicencio H., Rivas-Arancibia S. Effect of Chronic Oxidative Stress on Neuroinflammatory Response Mediated by CD4+T Cells in Neurodegenerative Diseases. Frontiers in Cellular Neuroscience. 2018, 12.

Stewart J. C., Janicki-Deverts D., Muldoon M.F., Kamarck T.W. Depressive symptoms moderate the influence of hostility on serum interleukin-6 and C-reactive protein. Psychosom. Med. 2008. 70, 197-204.

Strekalova T., Bahzenova N., Trofimov A., Schmitt-Böhrer A.G., Markova N., Grigoriev V., Zamoyski V., Serkova T., Redkozubova O., Vinogradova D., Umriukhin A., Fisenko V., Lillesaar C., Shevtsova E., Sokolov V., Aksinenko A., Lesch K.P., Bachurin S., 2018. Pro-neurogenic, Memory-Enhancing and Anti-stress Effects of DF302, a Novel Fluorine GammaCarboline Derivative with Multi-target Mechanism of Action. Mol Neurobiol. 55, 335-349.

Strekalova T., Couch Y., Kholod N., Boyks M., Malin D., Leprince P., Steinbusch H.M., 2011. Update in the methodology of the chronic stress paradigm: internal control matters. Behav Brain Funct. 27, 7-9.

Strekalova T., Spanagel R., Dolgov O., Bartsch D. Stress-induced hyperlocomotion as a confounding factor in anxiety and depression models in mice // Behav Pharmacol. 2005, 16: 171-80. 
Stronski S.M., Ireland M., Michaud P., Narring F., Resnick M.D. Protective correlates of stages in adolescent substance use: A Swiss National Study. J Adolesc Health. 2000, 26: 420-427.

Suarez E.C. Joint effect of hostility and severity of depressive symptoms on plasma interleukin-6 concentration. Psychosom. Med. 2003. $65,523-527$.

Suzuki K., Koizumi N., Hirose H., Hokao R., Takemura N., Motoyoshi S. Model of emotional stress in rats. Lab Anim Sci. 1998, 48: 353356.

Takahashi A., Flanigan M.E., McEwen B.S., Russo S.J. Aggression, Social Stress, and the Immune System in Humans and Animal Models. Front Behav Neurosci. 2018, 12: 56.

Takahashi A., Miczek K.A. Neurogenetics of Aggressive Behavior Studies in Rodents. Curr Top Behav Neurosci. 2014, 17: 3-44.

Terlecky S.R., Terlecky L.J., Giordano C.R. Peroxisomes, oxidative stress, and inflammation. World J Biol Chem. 2012, 3: 93-97.

Tsai S.J., Chiu H.J., Wang Y.C., Hong C.J. Association study of serotonin-6 receptor variant (C267T) with schizophrenia and aggressive behavior. Neurosci Lett. 1999, 271: 135-137.

Tsaluchidu S., Cocchi M., Tonello L., Puri B.K. Fatty acids and oxidative stress in psychiatric disorders. 2008. BMC Psychiatry 8: S5.

Tuvblad C., Baker L.A. Human Aggression Across the Lifespan: Genetic Propensities and Environmental Moderators. Adv Genet. 2011, 75: 171-214.

van Rijnsoever C., Sidler C., Fritschy J.M. Internalized GABA-receptor subunits are transferred to an intracellular pool associated with the postsynaptic density. Eur J Neurosci. 2005, 21: 327-338.

Veenema A.H., Blume A., Niederle D., Buwalda B., Neumann I.D. Effects of early life stress on adult male aggression and hypothalamic vasopressin and serotonin. Eur J Neurosci. 2006, 24: 1711-1720.

Vekovischeva O.Y., Aitta-Aho T., Echenko O., Kankaanpää A., Seppälä T., Honkanen A., Sprengel R., Korpi E.R. Reduced aggression in AMPA-type glutamate receptor GluR-A subunit-deficient mice. Genes Brain Behav. 2004, 3: 253-265.

Vignisse J., Sambon M., Gorlova A., Pavlov D., Caron N., Malgrange B., Shevtsova E., Svistunov A., Anthony D., Markova N., Bahzenova N., Coumans B., Lakaye B., Wins P., Strekalova T., Bettendorff L. Thiamine and benfotiamine prevent stress-induced suppression of hippocampal neurogenesis in mice exposed to 
predation without affecting brain thiamine diphosphate levels // Molecular and Cellular Neuroscience. 2017, 82: 126-136.

Waider J., Araragi N., Gutknecht L., Lesch K.P. Tryptophan hydroxylase-2 (TPH2) in disorders of cognitive control and emotion regulation: a perspective. Psychoneuroendocrinology. 2011, 36: 393-405.

Wei J., Liu W., Yan Z. Regulation of AMPA Receptor Trafficking and Function by Glycogen Synthase Kinase 3. J Biol Chem. 2010; 285: 26369-26376.

Weidner M.T., Lardenoije R., Eijssen L., Mogavero F., De Groodt L.P.M.T., Popp S., Palme R., Förstner K.U., Strekalova T., Steinbusch H.W.M., Schmitt-Böhrer A.G., Glennon J.C., Waider J., van den Hove D.L.A., Lesch K.P. Identification of Cholecystokinin by Genome-Wide Profiling as Potential Mediator of Serotonin-Dependent Behavioral Effects of Maternal Separation in the Amygdala. Front Neurosci. 2019, 13: 460.

Winkler D., Daher F., Wüstefeld L., Hammerschmidt K., Poggi G., Seelbach A., Krueger-Burg D., Vafadari B., Ronnenberg A., Liu Y., Kaczmarek L., Schlüter O.M., Ehrenreich H., Dere E., 2017. Hypersocial behavior and biological redundancy in mice with reduced expression of PSD95 or PSD93. Behav Brain Res. 352, 35-45.

Zhang Y.Q., Zhang C.S., Yuan G.Z., Yao J.J., Cheng Z.H., Liu C.J., Liu Q.H., Wan G.R., Shi G.Z., Cheng Y.R., Ling Y., Li K. Effect of Tryptophan Hydroxylase-2 rs7305115 SNP on suicide attempts risk in major depression. Behav Brain Funct. 2010, 6: 49. 


\section{Summary}

Our study revealed novel mechanisms of stress-induced aggression, highlighting the role of internalization of AMPA receptor subunits GluA1-3, increased expression of GluA1 and GluA3 subunits, decreased expression of GluA2 subunit and 5HT6 receptor in the limbic structures of the brain.

In the first study we used ultrasound stress model, where mice were exposed for 3 weeks to unpredictably alternating ultrasound frequencies that mimic their natural ultrasound "signals" of fear/anxiety. Ultrasound-exposed BALB/c mice demonstrated increased aggression scores in the residentintruder test (Chapter 2), as well as increased parameters of depressive-like and anxiety-like behaviors (Chapter 3). These changes were accompanied by increased GluA1-3 expression and internalization index and decreased expression of 5-HT6 receptor in the prefrontal cortex and hippocampus, suggesting implication of these molecules in the mechanisms of excessive aggression (Chapter 2, Chapter 3). Gene expression of neuroinflammatory markers TNF, IL-1B, IL-6, as well as a marker of distress, GSK$3 B$, was increased, and expression of neuroplasticity markers Ki67, PSD95, PSA-NCAM and B-catenin was decreased in both structures of the limbic system (Chapter 2, Chapter 3). We also showed elevated hippocampal concentrations of carbonyl protein, total glutathione and malondialdehyde, established markers of oxidative stress, in stressed mice; the latter parameter significantly correlated with most of investigated behavioral and 
molecular parameters (Chapter 2, Chapter 3). These data emphasize the role of oxidative stress in stress-induced aggression and suggest a potential for therapeutic use of antioxidants for its management in individuals with respective emotional disturbances. A 3-week administration of thiamine (vitamin B1) or its precursor benfotiamine (200 mg/kg/day), or herbal anti-oxidant has precluded an increase of aggression scores and other changes in emotionality, as well as most of molecular changes induced by the ultrasound exposure (Chapter 2, Chapter 3). Thus, our data have identified thiamine, benfotiamine and herbal anti-oxidant as effective treatments preventing overt aggression and associated behavioural and molecular aberrations.

In another study mice heterozygous for gene encoding the key enzyme of neuronal serotonin synthesis, Tryptophan hydroxylase-2 (Tph2-het), known to display a partial decrease of neuronal serotonin but normal social behavior, unlike highly aggressive Tph2 null mutant mice, were subjected to a rat exposure paradigm to study whether this stress can induce aggression in them. Predator stress induced aggressive behavior in Tph2-het mice, mimicking effects of Tph2-knock-out situation (Chapter 4). These changes were accompanied by decreased 5HT6 expression and altered concentrations of serotonin, its precursor 5-hydroxytryptophan, and the key metabolite, 5hydroxyindoleacetic acid, in the striatum, prefrontal cortex, hippocampus and dorsal raphe (Chapter 4). Hence, using two 
different models of aggression induced by stress, we identified new molecular correlates of aggression, associated with altered expression of AMPA receptor subunits decreased expression of 5HT6 receptor. This suggests that newly identified molecular correlates of aggression in our studies can be possible targets for pharmacological treatment of aggression in a clinic. 


\section{Samenvatting}

\section{Het begrijpen van de moleculaire mechanismen van}

agressie in BALB/c en TPH2-deficiënte muizen

Onze studie onthulde nieuwe moleculaire mechanismen van stress-geïnduceerde agressie in twee verschillende depressiemodellen te weten de veranderde expressie van AMPAreceptor subeenheden GluA2 en 5-HT6-receptor in de limbische structuren van de hersenen. Daarnaast toonden we de rollen aan van de internalisatie van de AMPA-receptor subeenheden GluA13 , de veranderingen in de expressie van deze subeenheden en de expressie van factoren van neuronale ontstekingen, oxidatieve stress en plasticiteit bij overmatige agressie bij muizen.

In de eerste studie gebruikten we een ultrasonoor stressmodel, waarbij de muizen gedurende 3 weken afwisselend werden blootgesteld aan onvoorspelbaar alternerende untrasonore frequenties die hun natuurlijke "signalen" van angst en vrees nabootsen.

De aan ultrasoon geluid blootgestelde $\mathrm{BALB} / \mathrm{c}$ muizen vertoonden verhoogde agressie scores in de resident-indringer test (Hoofdstuk 2), evenals verhoogde parameters van depressief en angstachtig gedrag (Hoofdstuk 3). Deze veranderingen gingen gepaard met verhoogde GluA1-3-expressie en internalisatieindex en een verminderde expressie van de 5-HT6-receptor in de prefrontale cortex en hippocampus.

Gen expressie van de neuro-inflammatoire markers TNF, IL-1B, IL-6, evenals de expressie van de marker van stress, GSK- 
$3 B$, waren toegenomen en de expressie van de markers voor neuroplasticiteit, Ki67, PSD95, PSA-NCAM en B-catenine waren in beide structuren van het limbisch systeem verminderd (Hoofdstuk 2, Hoofdstuk 3). We toonden ook verhoogde hippocampale concentraties van carbonyl-eiwit, totaal glutathion en malondialdehyde, vastgestelde markers van oxidatieve stress, aan bij muizen met door stress geïnduceerde agressie. De laatste parameter was significant gecorreleerd met de meeste onderzochte gedrags- en moleculaire parameters. Deze gegevens bevestigen de rol van oxidatieve stress in door stress geïnduceerde agressie. Een toediening gedurende 3 weken van thiamine (vitamine B1) of zijn voorloper benfotiamine (200 $\mathrm{mg} / \mathrm{kg} / \mathrm{dag}$ ), of een kruiden-antioxidant, voorkwam een toename van de agressiescores en de meeste moleculaire veranderingen ten gevolge van de blootstelling aan ultrasonoor geluid. Op basis van onze resultaten lijken thiamine, benfotiamine en een kruiden-antioxidant openlijke agressie en bijbehorende gedragsen moleculaire afwijkingen in muizen te voorkomen en dit suggereert dat deze middelen mogelijk gezien kunnen gaan worden als een effectieve behandelingen (Hoofdstuk 2).

In het tweede model werden muizen heterozygoot voor het gen dat codeert voor het sleutelenzym van neuronale serotoninesynthese, Tryptophan hydroxylase-2 (Tph2-het), onderworpen aan een blootstellingsstress van 5 dagen met ratten. Predatie-stress veroorzaakte overmatige agressie bij deze blootgestelde muizen die het gedrag van zeer agressieve Tph2 
null-mutante muizen nabootsten (bij deze laatsten ontbreekt hersenserotonine volledig). Tegelijkertijd vertoonde achtergrond beinvloeding bij deze muizen onderdrukking van de agressiviteit na stress. Aldus kan een gedeeltelijk tekort aan serotonine in de hersenen in combinatie met een stress ervaring openlijke agressie veroorzaken. Deze veranderingen gingen gepaard met verminderde expressie van de serotonine-receptor 5-HT6, een verhoogde expressie van AMPA-receptor subeenheid GluA2, een veranderd hersenmetabolisme van serotonine en zijn voorloper 5hydroxytryptophan en vaqn het metaboliet 5hydroxyindolazijnzuur (Hoofdstuk 4).

Met deze twee studies hebben wij nieuwe moleculaire correlaten van agressie geïdentificeerd welke geassocieerd zijn met een veranderde expressie van AMPA-receptor subeenheden en een verminderde expressie van 5-HT6-receptor. Dit suggeert aangrijpingspunten voor nieuwe potentiële therapieen. 


\section{Valorization}

\section{Relevance for society}

The World Health Organization categorizes aggression among the top 20 causes of disability. Negative social impact associated with the prevalence of violence and aggression is increasing, and with this rise is the need to understand the molecular changes that underpin aggressive behavior, and thus pharmacological methods of treatment.

In our work we identified new and validated molecular correlates of aggression that can be possible targets for pharmacological treatment of excessive aggression in the clinic. The expression of AMPA receptor GluA2 subunit was upregulated, while the expression of 5-HT6 receptor was downregulated in two different models of stress-induced aggression: an ultrasound model of emotional stress, and mice with partial deficit of the gene encoding tryptophan hydroxylase2 (TPH2), the key enzyme of the neuronal serotonin synthesis, which were subjected to rat exposure stress. We also demonstrated increased neuroinflammation, decreased hippocampal plasticity and oxidative stress to be associated with aggression in mice. Because of this, we studied antioxidant compounds thiamine (vitamin B1), its precursor benfotiamine, and plant extracts and identified them as effective treatments preventing excessive aggression and associated behavioral and molecular alternations, which have the potential to be used in the clinical practice. 
We also validated two new mouse models that result in excessive aggression. In the model of ultrasound-induced emotional stress mice are subjected to variable frequencies chosen to mimic natural ultrasonic signals of fear and anxiety without direct physical stressors. This approach enhances its etiological validity, since emotional stress is as a human-specific trait. Mice with partial genetic deficit of TPH2 develop excessive aggression after being subjected to predator, showing similar behavioral profile to that of null mutants, modeling sensitivity to stress of patients with specific TPH2 polymorphisms. Our data suggest that both models can be useful for drug researcher development, as they are effective, etiologically relevant and inexpensive.

\section{Target groups}

We consider our target group could be patients and individuals with excessive aggression, as well as researchers, working in the field of neurobiology of aggression.

\section{Activity / Products}

We proposed new ways of pharmacological management of excessive aggression, based on use of compounds with antioxidant properties. We also validated new aggression models with high etiological validity that could be used in drug researcher development.

\section{Innovation}

We have shown for the first time that the expression of 5HT6 receptor and GluA2 subunit of AMPA receptor was altered 
in two different models of aggression, suggesting these molecules as possible pharmacological targets of stress-related aggression. We proposed thiamine compounds and herbal antioxidant as new ways of treatment of excessive aggression and emotional behaviors. Moreover, we established and validated two new mouse aggression paradigms that model both genetic and environmental factors, such as emotional stress and TPH2 deficiency.

\section{Implementation}

Our results are relevant for both the scientific and medical communities. They have been or will be published in peerreviewed journals and presented at national and international conferences. 


\section{Acknowledgements}

First of all, I would like to thank Prof. Klaus-Peter Lesch for general supervision of my work that was a joint research project between Maastricht University, Department of Neuroscience and the I.M. Sechenov First Moscow State Medical University. I am very grateful to Prof. David Linden, head of School for Mental Health and Neuroscience, Faculty of Health, Medicine and Life Sciences, Prof. Jos Prickaerts, head of Division III, and administration of Maastricht University for their general support and help in organization of the defense of my $\mathrm{PhD}$ Thesis. In particular, I would like to thank Prof. Lesch for providing a unique opportunity to work in his labs in Germany and to have his support across the years 2016-2019. His attention to detail and gentle criticism helped me a great deal to improve my writing and reporting skills. I am especially thankful for his exceptional contribution to my stay and training in Wuerzburg University, and for important discussions and feedback regarding my research.

I express my special gratitude to Prof. Tatyana Strekalova, my day-to-day supervisor, who coached me since my start of the $\mathrm{PhD}$ project. She taught me general standards of research work, study design, planning and organization of experiments and quality control of behavioural experiments on mice. She also extensively trained me to analyze and present my data. Her encouragement and criticism helped me to contribute to 7 peerreview papers over the period of $\mathrm{PhD}$ training. With her 
invaluable organizational help and support, I was able to attend important international meetings and undertake traineeships in the most prominent laboratories in Europe that was crucial for my development as a researcher. During these joint activities and traineeships arranged by Prof. Strekalova, I acquired valuable behavioral and molecular techniques and carried out important experiments for my thesis. I am very thankful for her constructive advices and general support. The defense of my $\mathrm{PhD}$ Thesis would not be possible without her pivotal contribution to this process.

I am very thankful to Prof. Lucien Bettendorff, from whom I experienced the most warm welcome in the University of Liege, GIGA Neuroscience. Prof. Bettendorf supervised my work in his laboratory, helped me to learn HPLC and gave me invaluable advice concerning the methodology of my experiments as well as my scientific work in general. Thanks to his patient feedback and attention to my presentations, I have become more proficient in how to explain my results to an audience. Prof. Bettendorff has supported my work with his funds and taught me important techniques that became key methodologies used in $\mathrm{m} \mathrm{PhD} \mathrm{Thesis.}$ Next, I would like to thank cordially Prof. Daniel Anthony, who taught me the most important basics of brain sectioning, the main principles of scientific data interpretation and analysis, as well as other experimental skills and contributed to my knowledge of English. He provided invaluable help in preparing our papers and teaching me how to handle revisions of my papers. Thanks to him I was able to spend several weeks in his laboratory in Oxford 
University and interact with other researchers from his group. I am very grateful to Dr. Careen A. Schroeter, who greatly supported my experimental work and provided invaluable theoretical and methodological inputs. I would like to express my cordial appreciation to Dr. Johannes de Munter for providing important criticism to my work, contributing to data interpretation and teaching me the importance of working communication and presentation of my results.

I thank Dr. Joao Costa-Nunes for data discussion, practical contribution to my papers, and last but not least, his warm hospitality during my stay in Lisbon University. I greatly value his encouragement to perform my studies and helpful tips to carry on with my research activities. I thank Margaux Sambon from Liege University for teaching me how to master fluorometric analysis, and researchers the University of Würzburg: Gabriela Ortega, who taught me details of RT-PCR, Dr. Angelika G. Schmitt-Boehrer, Dr. Andrea Forero and Jann Kolter, who kindly helped me to learn immunohistochemistry, as well as Dr. Jonas Waider for his invaluable help in the HPLC analysis. I thank Jenna Hebert and Daniel Radford-Smith from Oxford University, for helping me during my traineeship there. I am very grateful to Prof. Alexei Umrukhin from the I.M. Sechenov First Moscow State Medical University for providing me constant help with research facilities. I am especially pleased to thank Prof. Andrey Svystunov, Prof. Denis Butnaru, Prof. Andrey Zamyatnin and Elina Vinogradova from the I.M. Sechenov First Moscow State 
Medical University for their invaluable help and support provided over the years in my work in Moscow.

On a separate note, I would like to thank my peers / friends I colleagues Dmitrii Pavlov, Ekaterina Veniaminova, Natalia Bazhenova, Dr. Natalia Markova, Dr. Alexander Trofimov, Diana Babevskaya and Margarita Oplatchikova who were young members of the Laboratory of psychiatric neurobiology in 20162018. Over the years, they have provided me with all kinds of practical assistance, moral support and wonderful companionship. I am very grateful to them for sharing great experiences during our joint experiments, travels and attendance of various kinds of trainings and conferences. I am happy that our joint work became very rewarding and that we will be allowed to defend our theses together with Dmitrii and Ekaterina.

Last but not least, I would like to address my warmest thanks to my mother Oxana, my dear grandmother Maria and my beloved husband Sergei, for supporting me across all these years and making me believe in my success, despite all challenges that came across. 


\section{About the author}

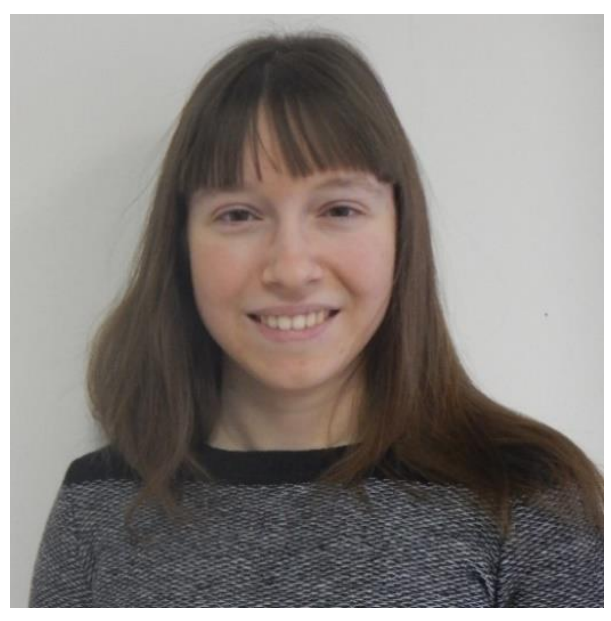

Anna Gorlova was born on October 29th, 1993 in Moscow, Russian Federation. Between 2009-2015 she studied physiology at the Faculty of Biology at Lomonosov Moscow State University. During her undergraduate studies, she was involved in research. In particular, she investigated cognitive features of thymectomized rats, as well as behavioral alterations in a mouse model of amyloidosis under the supervision of Dr. Anatoly Inozemtsev and Dr. Anna Novoseletskaya at the Laboratory of Evolution of Memory Mechanisms. After graduating in 2015 with an MS in biology, Anna studied the ultrasound model of emotional stress in rodents in Serbsky Institute of Psychiatry and Narcology and learned basic molecular techniques, such as RT-PCR and ELISA, under the supervision of Prof. Vladimir Chekhonin, Dr. Eugene Zubkov and Dr. Anna Morozova. In 2016 she continued her research as a junior researcher under the supervision of Prof. Tatyana Strekalova and Prof. Klaus-Peter Lesch in the laboratory of Psychiatric Neurobiology in the First Moscow State Medical University. In 2018 she was appointed as an external $\mathrm{PhD}$ student in the Department of Neuroscience, School for Mental Health and Neuroscience, Maastricht University, Netherlands. 
During her work on the PhD Thesis, coordinated by Prof. Strekalova as day-to-day supervisor and under general supervision of Prof. Lesch, Anna studied two paradigms that model both genetic and environmental factors of excessive aggression. She undertook her trainings at Laboratory of Neurophysiology, GIGA-Neurosciences of Liège University, Belgium, which she has visited six times, each time for several weeks in 2016-2020. During this traineeship, Anna learned to master immunofluorometric assays for different markers of oxidative stress, HPLC and Western blot analyzes under the supervision of Prof. Lucien Bettendorff and Margaux Sambon. In 2017 and 2018, Anna spent several weeks in the University of Würzburg, Division of Molecular Psychiatry, Center of Mental Health, where she learned immunohistochemical analysis and practiced RT-PCR under the supervision of Prof. Lesch, Dr. Andrea Forero and Gabriela Ortega. Anna had an opportunity to undertake training at the Department of Pharmacology in Oxford University, United Kingdom, where she performed behavioral analysis of SERT deficient mice and RNA isolation from brain samples of these animals. Under the supervision of Prof. Daniel C. Anthony she had a chance to practice brain sectioning for three weeks in 2018 in laboratories of Oxford University, as well as brain and spinal cord dissection and stereotactic brain injection of compounds in mice.

During her travels for various traineeships Anna gave numerous seminars and talks and had extensive data discussions 
with various experts and researchers, e.g. with Dr. Careen A. Schroeter and Dr. Johannes de Munter in Maastricht, with Prof. Bettendorff in University of Liege, Dr. Igor Pomytkin and Prof. Rainer Landgraf, Sechenov University, and Dr. Joao Nunes, Lisbon University. Over this time Anna has gained a sound experience in the development of study design, practical running of stress paradigms and analysis of aggression/dominance and various emotional and motor behaviors, conceptualizing and drafting papers and scientific presentations, as well as grant proposals. This work was largely done under the supervision of Prof. Strekalova. In Spring 2018, Anna also completed certified FELASA courses, category B, in Lisbon, Portugal. She attended international and local conferences, such as FENS Forum of Neuroscience in Berlin, Germany; EURON PhD student meeting in Kerkrade, the Netherlands; WPA World Congress of Psychiatry in Lisbon, Portugal; Inflammation and oxidative stress in translational research workshop in Estoril, Portugal; UK-Russia Young Medic Conference in Moscow, Russia; International "Stress and Behavior" Neuroscience and Biopsychiatry Conference in St.-Petersburg, Russia, and numerous other conferences, where she gave in total, 11 oral presentations and presented 8 posters. In addition, Anna is involved in the supervision of undergraduate students and contributes to the blog "Depression, aggression, and Vitamin B1 thiamine supplements as a new treatment" for the Eat2beNice project (Horizon 2020 EU Research and Innovation program, 
Grant No. 728018). In 2018 she was awarded with the 2 year research grant in a frame of Support of Young scientists by Russian Foundation of Basic Research for the project "A study of the role of oxidative stress in limbic structures of the brain in mouse models of stress-induced behavioral changes and its pharmacological management" (Grant No. 18-34-00275) and secured funding of $5300 \mathrm{EUR} /$ year up to 2020. She contributed to 8 papers with cumulative impact factor 28.2 in the following international peer-reviewed journals: Neuropharmacology, Stress, Progress in Neuro-Psychopharmacology \& Biological Psychiatry, Molecular and Cellular Neuroscience, Journal of Affective Disorders, European Neuropsychopharmacology, CNS Neuroscience \& Therapeutics and Acta Neurobiologiae Experimentalis, as well as to 9 papers in Russian journals. Her $\mathrm{H}$-index is currently 3 .

Tel: +7 (910) 4958492

E-mail: anna.gorlova204@gmail.com

\section{Employment:}

Since 01.11.2018 - PhD student in the Department of Neuroscience, School for Mental Health and Neuroscience, Maastricht University (0 fte).

Since 01.11.2016 - junior researcher at the Laboratory of Psychiatric Neurobiology, I.M. Sechenov First Moscow State Medical University (1 fte). 


\section{Presentations at domestic and international conferences:}

A. Gorlova, D. Pavlov, M. Sambon, K.P. Lesch, L. Bettendorff, T. Strekalova. Anti-stress effects of vitamin B1 (thiamine) compounds in new mouse model of emotional stress induced by ultrasound. 19th WPA World Congress of Psychiatry, Lisbon, Portugal, 23-24.08.2019 (poster presentation in English)

A. Gorlova. Ultrasound model of stress in mice and rats to induce neuroinflammation and oxidative stress: effects of diet, vitamin B1 and other interventions. Workshop Inflammation and oxidative stress in translational research: new models and treatments. Estoril, Portugal, 20.08.2019 (oral presentation in English)

A. Gorlova, D. Pavlov, M. Sambon, K.P. Lesch, L. Bettendorff, T. Strekalova. Molecular correlates of ameliorative effects of thiamine and benfotiamine on stress-induced aggression and brain oxidation in mice. UK-Russia Young Medic Conference, I.M. Sechenov First Moscow State Medical University, Moscow, Russia, 01.03.2019 (poster presentation in English)

A. Gorlova, N. Bazhenova, G. Ortega, N. Markova, T. Strekalova, K.P. Lesch, D.C. Anthony. Dynamic of aggressive behavior and gene expression during stress in Tph2 heterozygous mice. Poster session at the department of Normal Physiology, I.M. Sechenov First Moscow State Medical University, Moscow, Russia, 24.01.2019 (poster presentation in Russian)

A. Gorlova. Molecular correlates of stress-induced aggressiveness in mice during stress and genetic deficit of key enzyme of neuronal serotonin synthesis Tph2. Talk at I.M. Sechenov First Moscow State Medical University, Moscow, Russia, 20.11.2018 (oral presentation in English)

A. Gorlova, D. Pavlov. Effects of dibenzoyl thiamine in acute stress mouse models. Talk at GIGA-Neuroscience, University of Liege, Liege, Belgium, 12.11.2018 (oral presentation in English)

A. Gorlova, D. Pavlov, N. Bazhenova, M. Sambon, K.P. Lesch, L. Bettendorff, T. Strekalova. Thiamine and benfotiamine decrease brain oxidative stress in new mouse model of emotional stress with ultrasound exposure. 11th FENS Forum of Neuroscience, Berlin, 09.07.2018 (poster presentation in English) 
A. Gorlova. Effects of intracerebroventricular administration of Neuro-cells on motor hallmarks of amyotrophic lateral sclerosis in FUS-transgenic mice. 25th International "Stress and Behavior" Neuroscience and Biopsychiatry Conference, SaintPetersburg, 18.05.2018 (oral presentation in English)

N. Bazhenova, A. Gorlova, G. Ortega, N. Markova, D. Bonapartes, T. Strekalova, K.P. Lesch, D.C. Anthony. Brain biopsy as a new technique to address dynamics of gene expression during stress in mice. 25th International "Stress and Behavior" Neuroscience and Biopsychiatry Conference, Saint-Petersburg, 17.05.2018 (poster presentation in English)

A. Gorlova, D. Pavlov, N. Bazhenova, M. Sambon, K.P. Lesch, L. Bettendorff, T. Strekalova. Protective effects of treatment with thiamine (vitamin B1) and benfotiamine in the model of emotional stress induced by ultrasound. Sechenov International Biomedical Summit, 21.05.2018 (poster presentation in English)

A. Gorlova. Effects of the treatment with thiamine (vitamin B1) and benfotiamine in naïve and stressed mice. Talk at I.M. Sechenov First Moscow State Medical University, Moscow, Russia, 02.11.2017 (oral presentation in English)

A. Gorlova. Behavioral deviations preceding the onset of syndrome of amyotrophic lateral sclerosis: a study on transgenic mice with FUS-gene mutation. 19th EURON $\mathrm{PhD}$ student meeting, Maastricht University, Netherlands, 25.10.2017 (oral presentation in English)

E. Lysikova, A. Gorlova, N. Babushkina, J. de Munter, S. de Munter, K.P. Lesch, E. Wolters, D.C. Anthony, T. Strekalova. Behavioral deviations preceding the onset of syndrome of amyotrophic lateral sclerosis: a study on transgenic mice with FUS-gene mutation. 19th EURON $\mathrm{PhD}$ student meeting, Maastricht University, Netherlands, 25.10.2017 (poster presentation in English)

A. Gorlova, D. Pavlov, E. Zubkov, A. Inozemtsev, V. Chekhonin. Memory impairments and changes of BDNF gene expression in depressive-like state development. 30th ECNP Congress, Paris, France, 04.10.2017 (poster presentation in English)

A. Gorlova, D. Pavlov. Effects of the treatment with thiamine (vitamin B1) and benfotiamine in naïve and stressed mice. Talk at GIGANeuroscience, University of Liege, Liege, Belgium, 11.05.2017 (oral presentation in English)

A. Gorlova, D. Pavlov, E. Zubkov, A. Inozemtsev, V. Chekhonin. Memory impairments and changes of BDNF gene expression in 
depressive-like state development. IX International Congress "Biotechnology: perspectives of development", Moscow, Russia, 21-22.02.2017 (poster presentation in English)

A. Gorlova, D. Pavlov, M. Sambon, R. Shishorin, V. Chekhonin, K.P. Lesch, L. Bettendorff, T. Strekalova. Protective effects of treatment with thiamine (vitamin B1) and benfotiamine in the model of emotional stress induced by ultrasound. Poster session at the department of Normal Physiology, I.M. Sechenov First Moscow State Medical University, Moscow, Russia, 07.04.2017 (poster presentation in Russian)

A. Gorlova. Study of effects of Thiamine (B1) and DF302 on aggressive behaviour of Balb/c induced by 3-week ultrasound stimulation. Seminar at Maastricht University, Maastricht, Netherlands, 29.11.2016 (oral presentation in English)

A. Gorlova. Study of effects of Thiamine (B1) and DF302 on aggressive behaviour of Balb/c induced by 3-week ultrasound stimulation. Talk at GIGA-Neuroscience, University of Liege, Liege, Belgium, 28.11.2016 (oral presentation in English)

A. Gorlova. Protective effects of the treatment with thiamine (vitamin B1) and benfotiamine in the model of emotional stress induced by ultrasound. International Evaluation Conference of the Laboratory of Psychiatric Neurobiology, I.M. Sechenov First Moscow State Medical University, Moscow, Russia, 06.04.2017 (oral presentation in English).

A. Gorlova. Effects of Vitamin B1 (Thiamine) and new anti-Alzheimer drug DF302 on aggressive behaviour of mice in a ultrasound emotional stress model. International Conference at the I.M. Sechenov First Moscow State Medical University, Moscow, Russia, 16.12.2016 (oral presentation in English).

A. Gorlova. Dynamics of the development of depressive-like state in rats stressed by chronic exposure to ultrasound of variable frequencies. XXIII International conference of students and young researchers "Lomonosov" at the Lomonosov Moscow State University, Moscow, Russia, 11.09.2016 (oral presentation in Russian). 


\section{Funding Secured}

Grant of Russian Foundation of Basic Research for young investigators "My First grant": "A study of the role of oxidative stress in limbic structures of the brain in mouse models of stressinduced behavioral changes and its pharmacological management". (01.03.2018-01.03.2020, 5300 EUR/year.

\section{List of publications:}

Gorlova A, Ortega G, Waider J, Bazhenova N, Veniaminova E, Proshin A, Kalueff A, Anthony DC, Lesch KP, Strekalova T. Stressinduced aggression in heterozygous TPH2 mutant mice is associated with alterations in serotonin metabolism and expression of 5-HT6 and AMPA subunit 2A receptors. Journal of Affective Disorders (under revision).

Pavlov D, Gorlova A, Bettendorff L, Kalueff A, Umriukhin A, Proshin A, Lysko A, Landgraf R, Anthony DC, Proshin A, Strekalova T. Enhanced conditioning of adverse memories in the mouse modified swim test is associated with neuroinflammatory changes reversible by treatments with antidepressant properties. Neurobiology of Learning and Memory (under revision).

Costa-Nunes JP, Gorlova A, Pavlov D, Cespuglio R, Gorovaya A, Proshin A, Umriukhin A, Ponomarev E, Kalueff A, Strekalova T, Schroeter C. Ultrasound stress compromises the correlates of emotional-like states and brain AMPAR expression in mice: effects of antioxidant and anti-inflammatory herbal treatment. Stress (in press).

De Munter H, Lyundup A, Pavlov D, Walters E, Gorlova A, Veniaminova E, Umrukhin A, Kalueff A, Svistunov A, Kramer B, Lesch KP, Strekalova T. Neuro-Cell therapy improves motor outcomes and suppresses inflammation during experimental syndrome of amyotrophic lateral sclerosis in mice. CNS Neuroscience \& Therapeutics, 2019. 00, 1-14. 
Gorlova AV, Pavlov DP, Ushakova VM, Zubkov EA, Morozova AY, Zorkina YA, Inozemtsev AN, Chekhonin VP, 2020. Depressivelike state development in rats induced by chronic ultrasound exposure is accompanied by a decrease of gene expression of $\mathrm{GABA}_{A}$ receptor subunits in brain. Neurochemical Journal. 37, 1-8.

Gorlova A, Pavlov, D, Zubkov E, Zorkina Y, Inozemtsev A, Morozova A, Chekhonin V, 2019. Alteration of oxidative stress markers and behavior of rats in a novel model of depression. Acta Neurobiologiae Experimentalis. 79, 232-237.

Gorlova AV, Pavlov DA, Ushakova VM, Zubkov EA, Morozova AY, Zorkina YA, Inozemtsev AN, Chekhonin VP, 2019. Chronic exposure to ultrasonic frequencies results in selective increase of aggression level in rats. Doklady Biological Sciences. 486, 753755.

Gorlova A, Pavlov D, Anthony DC, Ponomarev E, Proshin A, Sambon M, Schmitt-Boeher A, Lesch KP, Bettendorff L, Strekalova T. Thiamine and benfotiamine counteract ultrasound-induced aggression, normalize AMPA receptor expression and plasticity markers, and reduce oxidative stress in mice. Neuropharmacology, 2019, 156: 107543.

Gorlova AV, Pavlov DA, Zubkov EA, Morozova AY, Inozemtsev AN, Chekhonin VP, 2019. Chronic ultrasound exposure leads to anhedonia, decrease of explorative activity and change of anxiety level in rats. Zhurnal Vysshei Nervnoi Deyatelnosti Imeni I.P. Pavlova. 69, 743-751.

Ushakova VM, Gorlova AV, Zubkov EA, Morozova AY, Zorkina YA, Inozemtsev AN, Chekhonin VP, 2019. Electroconvulsive therapy effect on anxiety in linear and non-linear rats with depressivelike disorder formed by ultrasound exposure. Bulletin of Experimental Biology and Medicine. 167, 424-427.

Pavlov D, Bettendorff L, Gorlova A, Olkhovik A, Kalueff A, Ponomarev $\mathrm{E}$, Inozemtsev A, Chekhonin $\mathrm{V}$, Lesch KP, Anthony DC, Strekalova T. Neuroinflammation and aberrant hippocampal plasticity in a mouse model of emotional stress evoked by exposure to ultrasound of alternating frequencies. Progress in Neuropsychopharmacology and Biological Psychiatry. 2019. 90, 104-116.

Ushakova VM, Gorlova AV, Zubkov EA, Morozova AY, Zorkina YA, Pavlov DA, Inozemtsev AN, Chekhonin VP, 2019. Experimental models of depressive disorder. Zhurnal Vysshei Nervnoi Deyatelnosti Imeni I.P. Pavlova, 69, 230-247. 
Gorlova AV, Pavlov DA, Zubkov EA, Morozova AY, Inozemtsev AN, Chekhonin VP. Three-week isolation does not lead to depressivelike disorders in rats. Bulletin of Experimental Biology and Medicine. 165, 1-3.

Vignisse J, Sambon M, Gorlova A, Pavlov D, Caron N, Malgrange B, Shevtsova E, Svistunov A, Anthony D, Markova N, Bahzenova N, Coumans B, Lakaye B, Wins P, Strekalova T, Bettendorff L. Thiamine and benfotiamine prevent stress-induced suppression of hippocampal neurogenesis in mice exposed to predation without affecting brain thiamine diphosphate levels. "Molecular and Cellular Neuroscience", 2017. 82, 126-136.

Ushakova VM, Zubkov EA, Morozova AY, Gorlova AV, Pavlov DA, Inozemtsev AN, Chekhonin VP. Effect of electroconvulsive therapy on cognitive functions of rats with depression-like disorders induced by ultrasound exposure. Bulletin of Experimental Biology and Medicine. 163, 599-601.

Gorlova AV, Pavlov DA, Ushakova VM, Zubkov EA, Morozova AY, Inozemtsev AN, Chekhonin VP, 2017. Dynamics of the development of depressive-like state in rats stressed by chronic exposure to ultrasound of variable frequencies. Bulletin of Experimental Biology and Medicine. 163, 296-298.

Pavlov DA, Gorlova AV, Ushakova VM, Zubkov EA, Morozova AY, Inozemtsev AN, Chekhonin VP, 2017. Effects of chronic exposure to ultrasound of alternating frequencies on the levels of aggression and anxiety in CBA and BALB/c mice. Bulletin of Experimental Biology and Medicine. 163, 409-411. 